\title{
Transport Properties of Rare Earth Nitrides
}

\author{
by \\ William Freeman Holmes-Hewett
}

\author{
A thesis \\ submitted to the Victoria University of Wellington \\ in fulfilment of the \\ requirements for the degree of \\ Doctor of Philosophy \\ in Physics.
}

Victoria University of Wellington 2020 



\begin{abstract}
In this thesis we investigate the transport properties of $\mathrm{SmN}, \mathrm{NdN}$ and $\mathrm{GdN}$, members of the rare earth nitride series of intrinsic ferromagnetic semiconductors. GdN is the central member of the series with seven occupied majority spin $4 f$ states and seven empty minority spin $4 f$ states. Both the filled and unfilled $4 f$ states are some few eV away from the conduction and valence band extrema, resulting in transport properties which are dominated by the extended Gd $5 d$ band. The half filled $4 f$ shell, with zero net orbital angular momentum, furthermore simplifies calculations and as such GdN is the most studied both experimentally and in theory. As one moves to lighter members, the filled $4 f$ states become unfilled states in the conduction band and the $4 f$ shell now has a net orbital angular momentum. Calculations concerning these members are now significantly more complicated, and as such there exists a wide range of predictions concerning the conduction band minima in the lighter rare earth nitrides. To inform the current theoretical and experimental literature we report on three studies concerning the transport properties of $\mathrm{SmN}, \mathrm{NdN}$ and GdN.

To begin we report on the anomalous Hall effect in $\mathrm{SmN}, \mathrm{NdN}$ and GdN. Under the symmetry of the rock-salt rare earth nitrides the magnitude of the anomalous Hall effect can imply the wave function of the conduction electron (i.e. $d$ or $f$ band). Measurements of the anomalous Hall effect in moderately doped samples are used to show the conduction channel in SmN and NdN is an $f$ band or hybridised $f / d$ band. Furthermore the sign of the anomalous Hall effect can be used to determine the orientation of the spin magnetic moment of the conduction electrons. Optical measurements of $\mathrm{SmN}, \mathrm{NdN}$ and $\mathrm{GdN}$ films are then reported. Optical measurements provide a probe of the band structure of a material via
\end{abstract}


direct transitions between the valence and conduction bands. Measurements of reflectivity and transmission on undoped $\mathrm{SmN}$ and $\mathrm{NdN}$ films were used to locate the unfilled majority spin $4 f$ bands which form the conduction band minima in each material.

Finally a preliminary study of heavily doped SmN, NdN and GdN is discussed. Structural measurements show a reduced lattice parameter while transport results find a significantly enhanced conductivity in heavily doped films. The Curie temperature is found to be enhanced and optical measurements show an increased absorption and red-shifted optical edge in doped films. The superconducting state of $\mathrm{SmN}$ is discussed and it is shown only to be present in moderately doped films, i.e. superconductivity is not present in undoped or degenerately doped $\mathrm{SmN}$, within our measurement limits. 


\section{Acknowledgements}

Much more than just an academic work, this thesis represents a period of my life where I feel I settled on who I am, and who I want to be. So, to the people mentioned below, please know these acknowledgements cover much more than the contents of this manuscript.

To begin, I thank my supervisors, Joe Trodahl, Bob Buckley and Ben Ruck. Without your assistance and patience this work would not have been possible. The extended discussions, spanning so much more than physics, has made this not only a rewarding but a truly enriching experience. These comments apply also to the other members of the rare earth nitride group, Franck, Jackson, Ali, Felicia, Eva, Sam, Denis, Jay, Simon, Tane and Catherine. The friendly and helpful people from Robinson, Shen, Guy, Yao and David who have assisted me time and again with equipment. Eric, Baptise, Chhayly, Matt, Adam, Matthias and Brendan from the Raman lab, who have put up with my distractions for four years. Campbell and Aleksa who have made the, sometimes difficult, jump from workmates to friends. Andy, Peter and David from the teaching labs, for all the equipment loans and electronics advise. Finally, Amanda, Casey and Poppit (the true experts) who have sat through, and commented on, nearly all my presentations over the last four years. Harris, who has arrived just in time to make the list, and my parents, for supporting and encouraging me to complete a PhD in physics, what seemed to be a truly bizarre idea at the time. 


\section{Contents}

1 Introduction $\quad 5$

1.1 Motivation ..................... 5

1.2 Outline ....................... 6

1.3 Publications ................. 7

2 The Rare Earth Nitrides 9

2.1 Theoretical Picture . . . . . . . . . . . . . . . 12

2.1.1 Electronic Structure . . . . . . . . . . . . . . . . . 12

2.1.2 Magnetic Properties . . . . . . . . . . . . . . . . . . 19

2.2 Resistivity, Hall effect and Optical Response . . . . . . . . 22

2.2.1 Resistivity and Hall effect . . . . . . . . . . . . . . . . 22

2.2.2 Optical Response . . . . . . . . . . . . . . 23

2.3 Experimental Picture . . . . . . . . . . . . . . . 26

2.3.1 Electrical Transport and Optical Measurements . . . 26

2.3.2 Magnetic Measurements . . . . . . . . . . . . . 28

2.4 Conclusions . . . . . . . . . . . . . . . . . . . . 29

3 Film Growth and Characterisation 31

3.1 Film Growth . . . . . . . . . . . . . . . . . . . 31

3.1 .1 Capping .................... 34

3.1.2 Substrate Selection, Film Geometry and Masking . . 37

3.2 Structural Characterisation . . . . . . . . . . . . . . . . 40

3.2.1 X-Ray Diffraction . . . . . . . . . . . . . 40 
3.2.2 SEM and Dektak . . . . . . . . . . . . . 45

3.3 Electrical Measurements . . . . . . . . . . . . . . . . . 47

3.3.1 Van der Pauw Geometry . . . . . . . . . . . . . . . . . 49

3.4 Magnetic Measurements . . . . . . . . . . . . . . . . . 51

4 The AHE in Rare Earth Nitrides 53

4.1 Theoretical Background .............. 53

4.2 Motivation . . . . . . . . . . . . . . . . . 60

4.3 Experimental Techniques . . . . . . . . . . . . . . 62

4.4 Results and Discussion . . . . . . . . . . . . . 66

4.4.1 Samarium Nitride . . . . . . . . . . . 66

4.4.2 Neodymium Nitride . . . . . . . . . . . . 73

4.4 .3 Gadolinium Nitride . . . . . . . . . . . . 76

4.5 Conclusions ..................... 81

5 Optical Properties of Rare Earth Nitrides 83

5.1 Motivation . . . . . . . . . . . . . . . . 83

5.2 Experimental Techniques . . . . . . . . . . . . . . 87

5.2.1 Measurement and Data Processing . . . . . . . . . 87

5.3 Reproducing Optical Measurements Using RefFit . . . . . . 98

5.4 Results and Discussion . . . . . . . . . . . . . . . . . . 102

5.4.1 Samarium Nitride . . . . . . . . . . . . . . 102

5.4 .2 Neodymium Nitride . . . . . . . . . . . . . . 110

5.4.3 Temperature Dependant Studies . . . . . . . . . . 114

5.5 Conclusions . . . . . . . . . . . . . . . . . 117

6 Properties of Doped Rare Earth Nitrides 119

6.1 Motivation . . . . . . . . . . . . . . . . . . . . . . 119

6.2 Experimental Techniques . . . . . . . . . . . . . . . . . 121

6.2.1 Estimating the Curie Temperature in $\mathrm{SmN}$. . . . . 121

6.2.2 Characterising the Superconducting State of SmN . . 123

6.3 Results . . . . . . . . . . . . . . . . . 125 
6.3.1 Structural Measurements . . . . . . . . . . . . 125

6.3.2 Optical Measurements . . . . . . . . . . . . . 127

6.3.3 Low Energy Behaviour of SmN . . . . . . . . . . 142

6.3.4 Magnetic Measurements and Superconductivity . . . 147

6.4 Conclusions . . . . . . . . . . . . . . . . . 155

7 Conclusions and Outlook 157

7.1 Recommendations for future work . . . . . . . . . . . 160 


\section{Chapter 1}

\section{Introduction}

\subsection{Motivation}

The rare earth nitride series of intrinsic magnetic semiconductors offers many avenues for investigation which span the topics of materials science. From an applied point of view, the rare earth nitrides, as dope-able, epitaxy compatible, magnetic semiconductors, are suited to implementation in spintronic devices [1]. Their strongly varying and contrasting magnetic properties offer a vast range of application in device structures as hard and soft, switch-able magnetic layers. They have many advantages over the dilute, magnetic semiconductors (i.e. (Ga,Mn)As) where the magnetism comes from the few \% of dopant ions, thus materials which are useful in a magnetic sense are necessarily heavily doped. The rare earth nitrides are intrinsically ferromagnetic due to their $4 f$ electrons and dope-able with electrons via nitrogen vacancy sites. In these materials the saturation magnetisation and electron concentration can now be independently controlled, which is a significant advantage over the standard dilute magnetic semiconductors.

The rare earth nitride series also shows promise as a new avenue in which to investigate localised electron systems. The fundamental physics of localised and strongly correlated electron systems has been a topic of 
intense interest since its identification, with many of the unusual properties exhibited still evading understanding. The essence of a strongly correlated electron system is the hybridisation of localised electrons (i.e. $f$ ), and more extended state electrons (i.e. $d$ ), in the band structure of a material. The vast majority of strongly correlated materials are metallic and have these hybridisations far from the Fermi energy, which is essentially out of reach in terms of electron transport. The rare earth nitrides now offer two advantages. They have $4 f$ bands which lie variously throughout the conduction and valence bands, many within a few $\mathrm{eV}$ of the conduction and valence band extrema, and furthermore, they are dope-able. The rare earth nitrides offer a series where the level of the $4 f$ in the conduction and valence bands can be selected via the choice of lanthanide element, and the Fermi energy can be tuned by doping with nitrogen vacancies. The combination of these properties allows the investigation of a great many hybridisation scenarios in both the conduction and valence bands.

For the full potential of exploitation of the rare earth nitrides to be realised the electronic properties must be understood in more detail than the current picture. Investigating these properties may also provide new information regarding the fundamental physics of strongly correlated electron systems.

\subsection{Outline}

The main aim of this thesis is to further the understanding of the transport properties of the rare earth nitrides, specifically GdN, SmN and NdN. This was achieved largely by experiment on the three materials listed.

To begin a discussion of the rare earth nitrides is given in Chapter 2. Here the electronic and magnetic properties are covered in regards to both theory and experiment. Chapter 3 then covers film growth and general characterisation measurements along with small investigations into the structure of the materials. The three results chapters follow. 
Chapter 4 covers measurements of the anomalous Hall effect in $\mathrm{SmN}$, $\mathrm{NdN}$ and GdN. Chapter 5 covers optical measurements on $\mathrm{SmN}$ and $\mathrm{NdN}$ and Chapter 6 discusses the properties of heavily doped rare earth nitrides. Each chapter begins with a brief review of the relevant literature and a description of specific experimental techniques pertinent to the measurements discussed. Chapter 4 has additionally some further background covering the basics of the anomalous Hall effect and the model used to describe this in the rare earth nitrides.

Finally, the results are summarised and an outlook and recommendations for further research are given.

\subsection{Publications}

A. Shaib, F. Natali, J. Chan, F. H. Ullstad, W. F. Holmes-Hewett, J. Miller, B. J. Ruck and H. J. Trodahl. Coexisting Structural Phases in the Catalytically Driven Growth of Rock Salt GdN, Mater. Res. Express, 7, 046404 (2020).

W. F. Holmes-Hewett, R. G. Buckley, B. J. Ruck, F. Natali and H. J. Trodahl. $4 f$ Conduction in the Magnetic Semiconductor NdN, Phys. Rev B., 100, 195119 (2019).

W. F. Holmes-Hewett, R. G. Buckley, B. J. Ruck, F. Natali and H. J. Trodahl. Optical Spectroscopy of SmN: Locating the $4 f$ Conduction Band, Phys. Rev B., 99, 205131 (2019).

W. F. Holmes-Hewett, F. H. Ullstad, B. J. Ruck, F. Natali and H. J. Trodahl. Anomalous Hall Effect in SmN: Influence of Orbital Magnetism and $4 f$-Band Conduction, Phys. Rev B., 98, 235201 (2018). 


\section{Chapter 2}

\section{The Rare Earth Nitrides}

This chapter begins by introducing the rare earth nitride materials and describing various important features in brief. The electrical and magnetic properties are then discussed in more detail, first the calculated band structure then the experimental picture. During this discussion the measurable physical properties relevant to this thesis are defined. Finally, based on the brief review given, areas of interest which are pertinent to this thesis are highlighted.

The rare earth nitrides crystallise in the rock-salt crystal structure, as shown in Figure 2.1. The structure is largely ionic comprising $\mathrm{N}^{3-}$ and $\mathrm{L}^{3+}$ ions owing to the large electro-negativity of $\mathrm{N} \sim 3$ compared to the relatively small electro-negativity of the $\mathrm{L}$ atoms $\sim 1.2$. The calculated lattice parameters of the rare earth nitrides span from $5.38 \AA$ in LaN to $4.87 \AA$ in LuN [2]. These are largely matched by experiment although variations have been noted with growth conditions $[3,4,5]$.

The rare earth nitrides were first investigated in the 1960s where several were found to order ferromagnetically. This ordering is unlike the other rare earth pnictides, which tend to find an anti-ferromagnetic order $[6,7,8,9]$. The rare earth nitrides, due to the gradual filling of the $4 f$ electron shell, have interesting and varied magnetic behaviour as one 


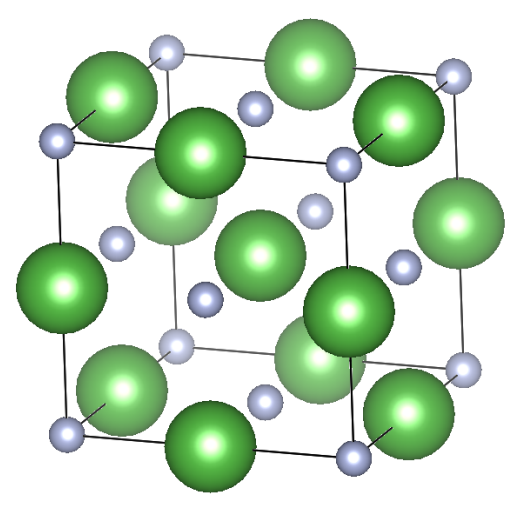

Figure 2.1: Schematic of the $\mathrm{NaCl}$ structure of the rare earth nitride materials. The large green circles represent the $\mathrm{N}^{3-}$ ions and the small blue the $\mathrm{L}^{3+}$ ions.

moves along the series. This is largely caused by the combination and competition of spin and orbital contributions to the magnetisation. The electrical properties of the rare earth nitrides showed significant variation, with early reports ranging from metallic to insulating $[1,8,9]$. What is clear is that a lack of stoichiometry will likely have effected early results from both the inclusion of nitrogen vacancies, which are thought to dope the material with electrons, and oxygen contamination which will quickly react with the material.

With modern ultra-high vacuum growth techniques, it is now possible to limit the contamination of films and dope the materials with nitrogen vacancies in a controlled fashion [1, 10,11]. This effectively allows one to tune the Fermi energy making the rare earth nitrides some of the few intrinsic ferromagnetic semiconductors. The significant spin-splitting in the conduction and valence bands of various rare earth nitride members furthermore ensures majority spin carriers alone up to moderate doping levels, for example, $\sim 1 \mathrm{eV}$ in $\mathrm{GdN}$ [12]. The rare earth nitrides, intrinsic ferromagnetic semiconductors, have significant advantages over the dilute magnetic semiconductors (DMS). The independent control of the Fermi energy, via varying the nitrogen pressure during growth, and the magnetic properties, though the choice of lanthanide, gives them great 
potential for application. This level of control is not yet found in the DMS, e.g. (Ga, Mn)As which must be doped heavily before its magnetic properties become useful [13].

Experiment has recently demonstrated epitaxial growth of both $\mathrm{GdN}$ and SmN on YSZ [14] and AlN templated Si substrates [15, 16]. As the film quality and fundamental understanding improve, it is not difficult to imagine the rare earth nitrides being integrated into devices such as magnetic memory elements and spin-filters, the first of which are already under development $[3,17]$. There is also ongoing work developing hetrostructures [18, 19, 20] and catalysis methods [11], the latter taking advantage of a remarkable ability allowing a clean lanthanide surface to break the molecular nitrogen bond.

The potential of the rare earth nitrides is by no means limited to application. They also provide an experimental springboard for the theoretical advancement of strongly correlated electron systems and density functional theory (DFT). The interplay of the extended L $5 d(\mathrm{~N} 2 p)$ states in the conduction (valence) band and the unfilled (filled) L $4 f$ states provides many different hybridisation scenarios in which strongly correlated behaviours can be studied. The choice of lanthanide element in the rare earth nitride can be used to select the energy of the $4 f$ levels relative to the valence or conduction band extrema [21, 22]. Furthermore, as these materials can be doped, it is possible to tune the Fermi energy to near the location of this hybridisation and observe the effect on the transport properties.

In the context of theoretical investigation, the simple structure of the rare earth nitrides reduces the size of basis sets required significantly speeding up calculations. This makes the comparison of theory and experiment more straight forward than in most other strongly correlated systems. The rare earth nitrides have recently been exploited in this regard as the test cases for a new class of pseudo-potentials for use in the DFT framework [23]. 
Even with the increase in interest in the rare earth nitrides in the last decade, the potential of these materials cannot be fully realised until the transport properties are more fully understood. There is still much uncertainty in regards to the location of the $4 f$ bands of various members and discrepancies between historical and modern results, which is almost certainly a result of nitrogen-vacancy contamination in the earlier work. This thesis aims to provide some guidance in these regards by locating the unfilled $4 f$ bands in some of the lighter rare earth nitrides and investigating the link between nitrogen-vacancy concentration and electron dynamics.

\subsection{Theoretical Picture}

In this section, the recent theoretical literature on the rare earth nitrides is briefly reviewed. This begins with a discussion of the electronic structure that is largely focused on GdN. This section also serves as a general introduction to the band structure of the rare earth nitrides. The magnetic properties are then covered. First, Hund's rules are introduced then, as with the electronic properties, the recent theoretical literature is briefly reviewed.

\subsubsection{Electronic Structure}

Various methods have been used to describe the electronic structure of the rare earth nitrides with one of the key differences between these methods being in the manner the $4 f$ electrons are treated. Here we begin with a description of the general band structure of the rare earth nitrides using GdN as an example, this is followed by a brief overview of the results found by different groups using various calculation methods. Due to a lack of theoretical studies on the band structure of doped rare earth nitrides this section is largely limited to discussion of the stoichiometric materials, the doped materials are briefly covered at the end of this section. 


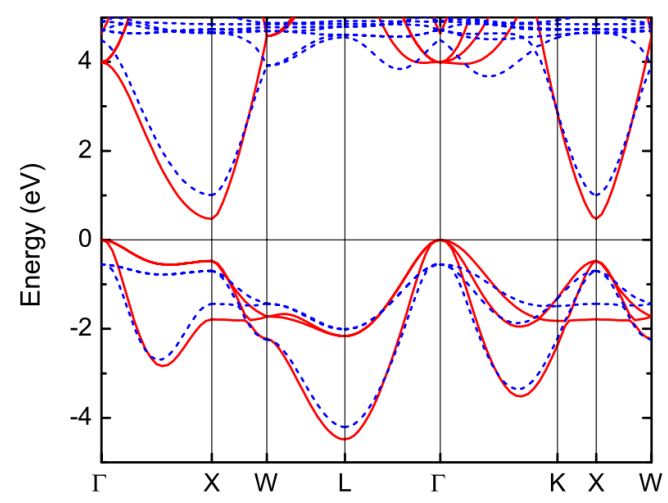

Figure 2.2: Calculated band structure of $\mathrm{GdN}$ using the $\mathrm{LSDA}+U$ method reproduced from reference [24]. The valence band is formed from N $2 p$ states with a maximum at $\Gamma$ and $\sim 0.5 \mathrm{eV}$ of dispersion before a local maximum at $\mathrm{X}$. The minimum optical bandgap is at X between the $\mathrm{N} 2 p$ valence bands and Gd $5 d$ bands, this gap has been adjusted to fit experiment.

In general, the highest energy valence bands in the rare earth nitrides are formed from N $2 p$ bands and / or the occupied L $4 f$ bands. The lowest energy conduction bands are generally formed from L $5 d$ and / or unfilled L $4 f$ bands. Many, particularly earlier, calculations have found the majority of the series to be either metallic or semi-metallic while recent experiments generally find a semiconducting ground state.

When discussing the electronic structure of the rare earth nitrides, GdN offers a good starting point. The half-filled $4 f$ shell results in a more simple band structure than many of the other members and greatly simplifies calculations. GdN provides a valuable comparison when considering the more complex members of the series due to this simple band structure which is, for the most part, agreed upon by theory and experiment. The following description of the electronic structure of $\mathrm{GdN}$ is based on the local spin density approximation with Hubbard $U$ correction $(\mathrm{LSDA}+U)$ calculations which have been adjusted to the experimental optical gap [2,24].

The calculated band structure for GdN is shown in Figure 2.2, the ma- 
jority and minority spin bands are shown in solid red and blue dashed lines respectively. The valence band is formed from N $2 p$ bands and has a maximum at $\Gamma$. It then decreases in energy towards a local maximum at $X$ before falling further towards $\mathrm{W}, \mathrm{L}$ and $\mathrm{K}$. There is only small spin splitting in the valence band with the majority spin sitting at slightly higher energy than the minority. Below the N $2 p$ bands are the seven filled majority spin $4 f$ states of $\mathrm{GdN}$. These occur over a small energy range near $-7 \mathrm{eV}$ well below the valence band maximum (not visible in the scale of the figure). It is these filled majority spin $4 f$ states which cause the spin splitting in the $\mathrm{N} 2 p$ bands, slightly lifting the majority spin above the minority (note that the exchange here is indirect, there is a direct ferromagnetic exchange between the $4 f$ and $\mathrm{Gd} 5 d$ bands, then anti-ferromagnetic exchange between the Gd $5 d$ and N $2 p$ bands [25]). Below the Gd $4 f$ bands are the $\mathrm{N} 2 s$ bands near $-12 \mathrm{eV}$ with the core levels far below. The conduction band is formed from a Gd $5 d$ band which, similar to the $\mathrm{N} 2 p$ valence band, is spin split to $\sim 1 \mathrm{eV}$ by the filled Gd $4 f$ states. The conduction band minimum is found at $\mathrm{X}$ with a direct bandgap of $\sim 1 \mathrm{eV}$ between the majority spin bands which, by construction, matches experiment. The indirect bandgap between $\Gamma$ and $X$ shown in this calculation is $0.43 \mathrm{eV}$.

The salient features of the GdN band structure, the valence band maximum at $\Gamma$ and a minimum optical gap at $X$ are qualitatively expected throughout the semiconducting members of the series. However, changes are seen in the calculated band structures by adding or removing electrons from the $4 f$ shell as one moves through the series [2].

For the lighter rare earth nitrides, there are now majority spin $4 f$ bands threading through and hybridising with the $\mathrm{L} 5 d$ conduction band. The location and hybridisation of these bands in various members is far from certain with different calculations ranging several $\mathrm{eV}$ in this regard when applied to the same material (EuN [2, 26, 27], SmN [2, 28, 29, 30] and $\mathrm{NdN}$ [2]). Moving to heavier rare earth nitrides, there are now filled minority spin $4 f$ bands threading and hybridising with the $\mathrm{N} 2 p$ valence 
band. The difficulty in calculating the band structures of the rare earth nitrides stems from the treatment of these localised $4 f$ electrons.

The first treatment of the electronic structure of the any of the rear earth nitrides appeared in a series of calculations on Gd-pnictides [31]. A semimetallic solution was found for $\mathrm{GdN}$, while metallic solutions were found for all other Gd-pnictides. Qualitatively the features for the band structure near the conduction band minimum and valence band maximum are similar to that of many more recent calculations. In this calculation, the valence band maximum is formed from a N $2 p$ band found at $\Gamma$ which falls towards a local maximum at $\mathrm{X}$ with a width of $\sim 0.6 \mathrm{eV}$ between these points. The conduction band minimum is formed from a Gd $5 d$ band at $\mathrm{X}$ with a $0.26 \mathrm{eV}$ optical gap at this point. This first calculation treats the filled $4 f$ as core levels and does not calculate the empty $4 f$ states at all. The LSDA method used in these DFT calculations is derived from a free electron gas model, so does not describe the localised $4 f$ states accurately without additional parameters. Due to this, it was initially easier to remove the $4 f$ states from the calculation altogether. To contrast this, the next method discussed was developed in part to remedy the atomic like treatment of $4 f$ electrons.

The self-interaction corrected local spin density (SIC-LSD) treatment removes the self-interaction of occupied states, which causes problems in general LSDA treatments of the strongly correlated $4 f$ electrons. This treatment has been applied to the entire rare earth nitride series by Aerts et al. [32]. They specifically treat the question of how many of the $4 f$ electrons need to be treated as localised states, effectively determining the valency of the ion and location of the $4 f$ states.

Aerts et. al performed calculations on all the rare earth nitrides and compared the ground state energy in both the di- and tri-valent forms. Their results showed that the trivalent ground state is preferred in all cases. The preference for the trivalent state is the strongest at the beginning and middle of the series, following the filling of the $4 f$ shell as one 
would expect. The differences in energy between the di- and tri-valent states in $\mathrm{NdN}, \mathrm{SmN}$ and EuN are small at $1.6 \mathrm{eV}, 1 \mathrm{eV}$ and $<0.1 \mathrm{eV}$ respectively.

In the lighter rare earth nitrides, the unfilled majority spin $4 f$ states are found very near the Fermi energy. These hybridise with the N $2 p$ causing the semi-metallic ground state. As the $4 f$ shell fills the unfilled majority spin $4 f$ states move to higher energy with less hybridisation with the $\mathrm{N} 2 p$ finally resulting in a semiconducting ground state in $\mathrm{TbN}$.

We now move to more recent calculations which use the $\mathrm{LSDA}+U$ method. The LSDA $+U$ method attempts to treat the $f$ electrons by adding a screened coulomb on-site repulsion, and a screened exchange interaction between the $f$ electrons. The coulomb repulsion effectively raises (lowers) unfilled (filled) $f$ states above (below) the Fermi energy while the exchange term tends to add width between these states in the band structure, i.e. a stronger exchange term in $\mathrm{GdN}$ will cause the seven filled $4 f$ states to occur over a wider range of energy in the valence band.

The most comprehensive treatment of the rare earth nitrides using the LSDA $+U$ method is by Larson et al. [2] which follows their earlier work on GdN and other Gd-pnictides [33]. Here the lowest energy state was found in two scenarios. In the first scenario, the cubic symmetry of the crystal is maintained when filling the $4 f$ shell. In the second the requirement of symmetry is relaxed, and instead a Hund's rules type scenario is used, maximising the orbital component of the total angular momentum $L_{z}$ (in fact a slight modification of Hund's rules, where $L$ is maximised). In most cases, the modified Hund's rules solution was found to minimise the total energy. Although for various members of the series the energies were similar so band structures for each scenario are presented. The largest advance in this series of calculations $[2,33]$ is the addition of both the $U_{d}$ and $U_{f}$ parameters to all members of the series.

Larson et al., unlike the earlier calculations, find most of the series as semiconducting with only PrN, PmN and SmN semi-metallic. The finite 
bandgaps are largely caused by the addition of the Hubbard $U$ parameter, or more specifically the two $U_{d}$ and $U_{f}$ parameters, and to a lesser extent the $J_{d, f}$ parameters. The $U_{f}$ parameter effectively adjusts the splitting between the empty and filled $4 f$ states, which is determined by scaling $U_{f}$ until the calculation fit experimental photoemission data for Gdpnicitdes $[2,33,34]$. The $U_{f}$ term will change throughout the series as the $4 f$ shell fills and thus was scaled for all other members in the calculations. $J_{f}$ determines the strength of the exchange interaction between filled or unfilled states and can be directly calculated $[2,33]$. The $U_{d}$ parameter is similar to $U_{f}$ but now lifts the empty $5 d$ states relative to the filled $5 d$ states. Although the result is similar, the underlying physics is somewhat different between the $U_{f}$ and $U_{d}$ terms. The $d$ states in the semiconducting rare earth nitrides are over screened by the free electron gas used in the LSDA calculations. This screening lowers the energy of the $5 d$ states, thus the $U_{d}$ parameter is used to lift these back up to the experimental level [2, 24, 33].

In these calculations $[2,33] U_{d}$ was adjusted to fit the current experimental bandgap of $0.98 \mathrm{eV}$ in the paramagnetic phase of GdN [35]. This same $U_{d}$ parameter was used for all members of the series, while $J_{d}$ was taken as zero. It should be noted that a more recent LSDA $+U$ calculation of the GdN band structure [24], which was discussed earlier and shown in Figure 2.2, has been completed with a $U_{d}$ parameter to match much more recent optical measurements which resulted in a bandgap of $\sim 1.3 \mathrm{eV}$ in the paramagnetic phase [24]. Calculations for other members of the series have yet to be updated with this $U_{d}$ value.

The most rigorous approach to band structure calculations applied to the rare earth nitrides is the GW method. This method is all-inclusive in that core and valence exchange is included without the need for additional parameters. The GW method has been shown to reproduce the bandgaps in standard semiconductors accurately and has been applied to GdN [36] and other rare earth nitrides $(\mathrm{SmN}, \mathrm{DyN}, \mathrm{HoN}, \mathrm{YbN})$ [37]. In general, the GW method applied to the rare earth nitrides does accurately reproduce 
the experimental optical bandgaps without the need for empirical parameters such as $U_{d}$ and $U_{f}$. The placement of the $4 f$ bands in the valence band is also in agreement with $\mathrm{x}$-ray photoemission spectroscopy studies $[38,39]$. The unfilled $4 f$ levels in the conduction band are significantly lifted over the LSDA $+U$ calculations, the overestimation of the energy of unfilled $f$ levels is a known issue regarding the GW method.

It is interesting to compare the results of the calculation methods used. The SIC-LSD method resulted in largely semi-metallic solutions with the $4 f$ bands contributing largely to the density of states at the Fermi energy. The LSDA $+U$ calculations were then largely more consistent with the experimental picture with only three of the members of the series remaining as semi-metallic. These calculations also placed the $4 f$ levels further from the conduction band minimum and valence band maximum. Finally, the GW calculations find only $\mathrm{SmN}$ to be semi-metallic and place the unfilled $4 f$ levels further still from the conduction band minimum.

The previous discussion relates to the stoichiometric materials where much of the theoretical literature is focused, however, there is importance in considering the nitrogen-deficient materials. There is only a single calculation of the electronic properties of any of the rare earth nitrides, GdN, concerning specifically the effect of nitrogen vacancies on the band structure [40]. Here band structure calculations are completed using LSDA $+U$ with both $U_{f}$ and $U_{d}$ parameters, as described above. The calculations use a rock-salt structured supercell of 64 atoms with a vacancy concentration of $1 / 32$. The calculated band structure now has three additional impurity states for the three electrons corresponding to each vacancy. Two of these impurity states exist in the gap between the valence and conduction bands, these are completely filled and have minimal dispersion. The final impurity state hybridises with the Gd $5 d$ conduction band, effectively raising the Fermi energy into the $5 d$ band to $\sim 0.7 \mathrm{eV}$. The picture here is of a heavily doped material, with a carrier concentration of $\sim 10^{21} \mathrm{~cm}^{-3}$ where the Fermi energy lies within the hybridised conduction / impurity band. 


\subsubsection{Magnetic Properties}

\section{Hund's Rules}

The magnetic properties of the rare earth nitrides stem largely from the $4 f$ electrons. The combination of spin and orbital magnetism as the $4 f$ shell fills along the series gives the rare earth nitrides interesting and varied magnetic behaviour. In a simple sense one can gain a qualitative understanding of the magnetic properties of the rare earth nitrides by considering Hund's rules in the $4 f$ shell.

The atomic like properties of $4 f$ electrons are caused by their localised nature, specifically the small radius of the $4 f$ wave function. This localised character implies a strong Coulomb interaction between the electrons in the $4 f$ shell and reduces the effects of orbital quenching common to the transition metals. The electron-electron Coulomb interaction dominates the spin-orbit interaction implying the $\mathbf{L} \cdot \mathbf{S}$ coupling scheme is valid over the $\mathbf{J} \cdot \mathbf{J}$ which becomes dominant in heavier materials. In this regime the ground state of an ion can be given by the empirical Hund's rules, which determine the lowest energy configuration in three simple steps. The first two rules are related to the Coulomb interaction and Pauli exclusion principle, while the third is related to the spin-orbit interaction.

- Rule one: The total spin quantum number $S$ is maximised such that as electrons are successively added to a shell they are all of parallel spin until the number of electrons $n>2 l+1$ at which point successive electrons will have anti-parallel spin.

- Rule Two: The total orbital angular momentum $L$ should be maximised while being consistent with the first rule and the Pauli exclusion principle.

- Rule Three: If the shell is less than half filled the total angular momentum $J=|L-S|$, whereas if the shell is more than half-filled 
$J=|L+S|$. This is essentially stating that the spin and orbital angular momenta are anti-parallel for less than half-filled shells and parallel for more than half-filled shells.

These three rules describe how electrons can be successively added to a shell to minimise the total energy. The Hund's rules ground state then predicts a magnetic moment of $M=\mu_{B}\left|L_{z} \pm 2 S_{z}\right|$ where $\mu_{B}$ is the Bohr magneton. The simple application of Hund's rules neglects other contributions, such as the crystal field [41], which have been shown to cause considerable effects in SmN [42] and NdN [43]. Furthermore, in the $\mathbf{L} \cdot \mathbf{S}$ coupling scheme the stationary states of the Hamiltonian are $L^{2}$ and $S^{2}$ rather than $L_{z}$ and $S_{z}$.

As an example of the application of Hund's rules we consider the case of $\mathrm{NdN}$ with electronic configuration $4 f^{3}$. Rules one and two state that the spin and orbital moments should be maximised thus $S=3 / 2$ and $L=6$. Rule three tells us that in the less than half-filled shell $S$ and $L$ are antialigned thus $J=9 / 2$ and is dominated by the orbital component. The total magnetisation, in the ferromagnetic state, is then $M=|L-2 S| \mu_{B}=3 \mu_{B}$ and again in the orbital direction. Another interesting example is the case of $4 f^{5} \mathrm{SmN}$, where the application of Hund's rules results in a zero total magnetisation.

\section{Theoretical Picture}

There have been various theoretical studies on the ferromagnetic state of the rare earth nitrides, most of which focus on GdN, again due to the simplicity offered by the half-filled $4 f$ shell. Various calculations $[2,44,45]$ all agree on total moment per $\mathrm{Gd}^{3+}$ ion as $\sim 7 \mu_{B}$. This generally involves a near-zero orbital contribution, as qualitatively expected from Hund's rules, and a small opposing contribution of $\sim 0.1 \mu_{B}-0.01 \mu_{B}$ from the $\mathrm{N}^{3-}$ ions. There is much less agreement, however, on the Curie temperature with calculations ranging from $8 \mathrm{~K}$ [25] to $67 \mathrm{~K}$ [33]. 
To understand why calculations can show such strong agreement on the saturation magnetisation while such little agreement on the Curie temperature we can, once again, consider the $4 f$ shell. It is clear that the majority of the magnetisation is due to alignment of the $4 f$ electrons, however, the inter-atomic exchange is much less clear. The small radius of the $4 f$ wave function rules out any direct $f-f$ coupling. Given the many similarities between $\mathrm{EuO}$ and $\mathrm{GdN}$, it was initially suspected that the inter-ion Gd-Gd exchange may also be similar to the exchange in EuO. The much lower energy of the occupied $4 f$ levels in $\mathrm{GdN}$, however, rule out the various scenarios pertinent to $\mathrm{EuO}$ where the filled $4 f$ lies much closer to the unfilled $5 d$ bands $[46,47]$. One possible scenario is that the exchange is mediated by the induced moments on the Gd $5 d$ and N $2 p$ orbitals [25]. Here there is ferromagnetic on-site $f-d$ exchange, then anti-ferromagnetic $d-p$ exchange between the $\mathrm{Gd}$ and $\mathrm{N}$ ions, this chain linking the $\mathrm{Gd}$ ions in the lattice. The exchange between the localised $4 f$ moments in GdN is clearly complex and furthermore, there is experimental evidence of a strong effect of nitrogen vacancies on the exchange mechanism [48, 49].

Theoretical studies have attempted to address the contribution of nitrogen vacancies on the exchange. However, have still not resolved the common experimental Curie temperature of $\sim 70 \mathrm{~K}$ in heavily doped films [ 40 , 47]. The situation is made even more complex as the inclusion of nitrogen vacancies is also thought to affect the structure of the material, including the average lattice spacing $[3,5]$. Studies have shown that the magnetic properties are also sensitive to the lattice parameter with the nearest-, next nearest- and third nearest- neighbour exchange parameters all varying independently with lattice parameter [50].

Although much less numerous than GdN there exist theoretical studies of the magnetic properties of $\mathrm{SmN}[2,42,51]$. SmN has a much more complex magnetic behaviour than GdN caused largely by the less than half-filled $4 f$ electron shell. In $\mathrm{SmN}$ the $4 f$ shell now carries a non-zero orbital component to the magnetisation which all theoretical studies find 
opposes the spin component. This opposition then results in a near cancellation between the spin and orbital components of the magnetisation. Two studies using LSDA $+U$ find the net magnetisation is slightly dominated by the spin component $[2,51]$. A first-principles study, which directly diagonalises the $4 f$ Hamiltonian, finds a slightly orbital dominated net magnetisation [42] with partial quenching of both the spin and orbital components, which is in-line with experimental reports $[19,52]$.

\subsection{Resistivity, Hall effect and Optical Response}

Up until this point few directly measurable transport properties of the rare earth nitrides have been discussed. The band structure itself is almost never directly measured in the form pictured in Section 2.1.1. Thus before the current experimental literature is considered, we will briefly pause discussion of the rare earth nitrides and cover the more commonly measured physical properties relating to this thesis.

\subsubsection{Resistivity and Hall effect}

The properties of charge carriers at the Fermi level at any given temperature can, in a sense, be probed directly via measurements of the resistivity. This is often in the context of a simple Drude picture where the DC conductivity can be written as

$$
\sigma(n, \tau)=\frac{n e^{2} \tau}{m^{*}}=\frac{1}{\rho(n, \tau)},
$$

where $n$ is the carrier concentration, $e$ is the electronic charge, $\tau$ is the scattering time and $m^{*}$ is the effective mass. The conductivity is the inverse of the resistivity $\rho(n, \tau)$ which is itself defined as the physically measurable resistance $R(n, \tau)$ of a material multiplied by the appropriate dimensions.

The carrier concentration $n$ is measured via the Hall effect, which is apparent in a material under crossed electric and magnetic fields where 
charge carriers are subject to the Lorentz force. The Lorentz force tends to deflect charge carriers towards one side of a conductor until it is balanced by an induced electric field [53]

$$
E_{h}=\frac{e \tau}{m} \frac{1}{\sigma} B J .
$$

This lets us define the transverse Hall resistivity $\rho_{h}$ as

$$
\rho_{h}=\frac{e \tau}{m} \frac{1}{\sigma} B=\frac{1}{n e} B .
$$

With the above we see that in the simple case of a single band free-electron system the Hall resistivity is inversely proportional to the carrier density $n$ and the electronic charge $e$, and should be linear in the applied field $B$. In this sense, one can not only measure the concentration of charge carriers but also the sign of the carriers by a plot of the Hall resistivity as a function of the applied field.

\subsubsection{Optical Response}

The optical response of a material can yield information regarding charge carriers at the Fermi energy (if present) and vertical transitions between filled and unfilled bands on a band structure diagram. In a classical oscillator model [54] an electron and nucleus are coupled together as a dipole with resonant frequency $\omega_{0}$. The interaction with an electric field of frequency $\omega$ introduces forced oscillations into the dipole. The equation of motion of the system can be solved to find the dielectric function for this single oscillator. A real optical medium with electron density $n$ can, in this model, be represented by a series of such oscillators. The resulting dielectric function can then be written as the sum of these

$$
\epsilon(\omega)=1+\frac{n e^{2}}{\epsilon_{0} m_{0}} \sum_{j} \frac{1}{\omega_{0, j}^{2}-\omega^{2}-i \gamma_{j} \omega},
$$


where $m_{0}$ is the electron mass, $\epsilon_{0}$ is the vacuum permittivity and $\gamma$ is the damping rate of the restoring force. Metallic systems or doped semiconductors will furthermore have free electrons. For these unbound electrons the above argument is slightly changed in that there is no restoring force. Solving the equation of motion then results in the dielectric function, or AC conductivity,

$$
\epsilon(\omega)=1-\frac{n e^{2}}{\epsilon_{0} m_{0}} \frac{1}{\omega^{2}+i \gamma \omega}, \quad \sigma(\omega)=\frac{\sigma_{D C}}{1-i \omega \tau},
$$

where $\sigma_{D C}$ is defined in Equation 2.1. We now move to a quantum model of the interaction of an electromagnetic wave with a solid.

An optical transition is generally the excitation of an electron from one energy band to another via the absorption of a photon of energy $\hbar \omega$ where the final and initial states are of the same momentum $k$. For such a transition to be the case, there must exist an electron in the initial state $\psi_{i}$, the Pauli exclusion principle then gives that the final state $\psi_{f}$ must be empty. The typical example of such a transition is the excitation of an electron from the valence band, below the Fermi energy, into the empty conduction band of an undoped semiconductor. An excitation such as this requires the photon carry energy

$$
E_{f}=E_{i}+\hbar \omega
$$

where $E_{f}$ and $E_{i}$ are the energies of the final and initial states respectively. When such transitions happen in a solid with continuous energy bands we must consider the density of electronic states at both the initial and final energies; this is done most conveniently by using the joint density of states $g(\hbar \omega)$. We can then write the transition rate from $\psi_{i}$ to $\psi_{f}$, using Fermi's golden rule, as

$$
W_{i \rightarrow f}=\frac{2 \pi}{\hbar}|M|^{2} g(\hbar \omega)
$$

where $|M|$ is the matrix element concerning the transition between $\psi_{i}$ and 
$\psi_{f}$. The matrix element can be calculated using the overlap integral in the dipole approximation. The direct link to the band structure of the material can be seen in the term $g(\hbar \omega)$ which is inversely proportional to $\nabla_{k}\left(E_{f}-\right.$ $\left.E_{i}\right)$. This gives that the transition rate is very high at points where the conduction and valence bands track one another, in other words where $\nabla_{k}\left(E_{f}-E_{i}\right)$ is $\sim 0$.

Finally, we want to relate this transition rate $W_{i \rightarrow f}$ to the optical constants. The absorption coefficient $\alpha$ is defined as the power removed from the incident beam per unit volume per unit incident flux of electromagnetic energy. This can be written using the transition rate $W_{i \rightarrow f}$ and the Poynting vector $S$ as

$$
\alpha(\hbar \omega)=\frac{\hbar \omega W_{i \rightarrow f}}{S}, \quad S=\frac{\omega}{8 \pi} \frac{n_{r} \omega}{c}
$$

where $n_{r}$ is the real part of the refractive index. From here imaginary part of the dielectric function can be calculated as

$$
\epsilon_{2}=\alpha \frac{n_{r} c}{\omega}
$$

which is now free from the dependence on the refractive index and can be determined from the band structure alone. The real part of the dielectric function can now be calculated using the Kramers-Kronig relationship as

$$
\epsilon_{1}=1+\frac{2}{\pi} P \int_{0}^{\infty} \frac{\omega^{\prime} \epsilon_{2}\left(\omega^{\prime}\right)}{\omega^{\prime 2}-\omega} d \omega^{\prime}
$$

where $P$ denotes that the principal value of the internal is taken. The optical conductivity $\sigma(\omega)$ can then be defined via

$$
\epsilon(\omega)=\epsilon_{0}+i \frac{\sigma(\omega)}{\omega}
$$

where $\epsilon(w)=\epsilon_{1}+i \epsilon_{2}$. 


\subsection{Experimental Picture}

\subsubsection{Electrical Transport and Optical Measurements}

In contrast to the mixed reports in the early literature $[6,7,8,9]$, more recent measurements have confirmed the semiconducting nature of rare earth nitride films via resistivity and optical measurements.

Measurements of the resistance taken in-situ during film growth for both GdN [10] and SmN [55] show an enhanced conductivity when films were prepared at a reduced nitrogen pressure. The left-hand panel of Figure 2.3 shows these measurements. It is important to note that the nitrogen-vacancy concentration is never directly measured. The reduced resistivity and enhanced n-type carrier concentration of films grown at a reduced nitrogen pressure imply that electrons are doped via the inclusion of nitrogen vacancies. The doping of electrons via nitrogen vacancies is furthermore in-line with the calculation previously discussed concerning nitrogen-deficient $\mathrm{GdN}$ [40]. It is then reasonable to expect the doping mechanism is similar in other members of the series.

The temperature-dependent resistivity of rare earth nitride samples has also shown behaviour characteristic of semiconducting materials. The right-hand panel of Figure 2.3 shows the resistivity of a GdN film as a function of temperature from $300 \mathrm{~K}$ to $\sim 2 \mathrm{~K}$ [10]. In the high-temperature regime, above $100 \mathrm{~K}$, the resistivity is non-metallic, increasing slowly as temperature decreases, consistent with the picture of a heavily doped semiconductor. At lower temperatures there is a peak in the resistivity related to the magnetic transition. As the material orders below the Curie temperature there is a reduction in spin-dependent scattering leading to a reduction in the resistivity. The majority spin band which forms the conduction band minimum in Figure 2.2 is also expected to fall in energy as the sample moves from the paramagnetic to the ferromagnetic phase. The decreasing energy of this band results in an enhanced carrier concentration, further reducing the resistivity. Finally, at the lowest temperatures, the resistivity 

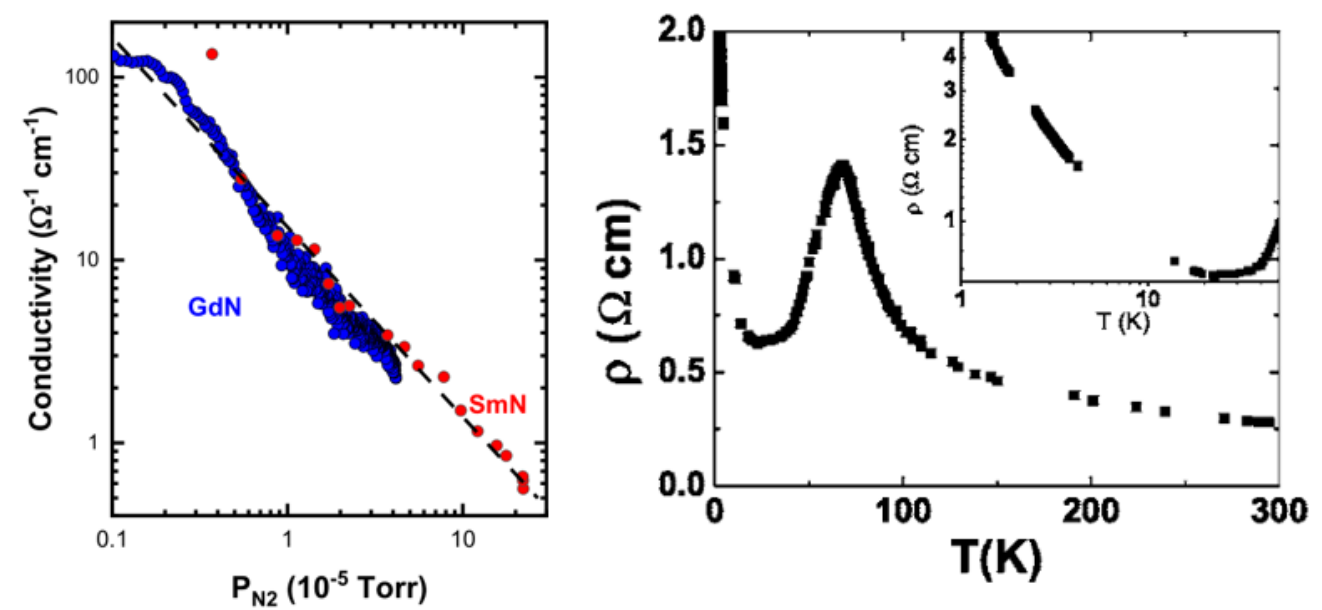

Figure 2.3: Left: Measurement of the conductivity as a function of pressure in GdN (blue) and SmN (red) reproduced from reference [55]. Right: Measurement of the resistivity as a function of temperature for a $\mathrm{GdN}$ sample, reproduced from reference [10].

appears to diverge indicating an absence of extended states at the Fermi energy. As shown in the left-hand panel of Figure 2.3 the conductivity of a film can be changed significantly by the growth conditions. This will in-turn alter the nature of the temperature dependence of the resistivity with degenerately doped films taking a positive temperature coefficient of resistance. Measurements of the resistivity as a function of temperature showing a similar non-metallic behaviour as seen for GdN in Figure 2.3 have been seen in $\mathrm{DyN}$ [56], $\mathrm{NdN}$ [43], YbN [57] and SmN [58].

In regards to less general transport behaviour, GdN, SmN and $\mathrm{NdN}$ have seen recent interest in their anomalous Hall effect. A semi-classical description developed for the case of GdN has found quantitative agreement with experiment [12]. Similar descriptions were then applied to $\mathrm{SmN}$ [59] and $\mathrm{NdN}$ [22] and used to show the influence of the $4 f$ levels on the conduction channels in both materials.

$\mathrm{SmN}$ stands out in regards to its transport properties as below $\sim 5 \mathrm{~K}$ it enters a superconducting state when appropriately doped [58]. The na- 
ture of the superconductivity is not completely understood. Still, it is suggested to involve the heavy-mass $4 f$ band.

Optical measurements have been conducted on various members of the rare earth nitride series. GdN is the most thoroughly studied where a $1.3 \mathrm{eV}$ bandgap has been found at room temperature, which reduces to $\sim 1 \mathrm{eV}$ in the ferromagnetic phase $[24,60]$. In addition to the redshift of the absorption onset the development of an additional contribution to the absorption at $\sim 1.7 \mathrm{eV}$ in low-temperature measurements signals transitions between the minority spin bands at the $X$ point [61]. Optical measurements of DyN [62], NdN [22, 43], YbN [57], EuN [27] and $\mathrm{SmN}$ [21, 63] have all found bandgaps near $1 \mathrm{eV}$ and at most weak indications of free carriers in moderately doped samples.

\subsubsection{Magnetic Measurements}

The experimental literature on the magnetic behaviour of the rare earth nitrides is again dominated by GdN. Typical results yield a saturation of $\sim 7 \mu_{B}$ in-line with calculations $[10,15,49,60,64]$, however, there are reports of a reduced saturation magnetisation in films with expanded lattice parameters [3,4]. Curie temperatures are generally reported between $50 \mathrm{~K}$ and $70 \mathrm{~K}$ in the above studies, however, it is possible to increase the Curie temperature to as high as $\sim 200 \mathrm{~K}$ by heavily doping films with nitrogen vacancies [48]. More recent experimental results now indicate the presence of two phases in ferromagnetic GdN [5]. These phases are identified as GdN I, the stoichiometric intrinsic form with a Curie temperature of $\sim 50 \mathrm{~K}$ and GdN II, a vacancy rich phase, with an enhanced Curie temperature of $\sim 70 \mathrm{~K}$.

$\mathrm{SmN}$ has also seen various experimental studies due to the interesting near cancellation between the spin and orbital components of the magnetisation in the $4 f$ shell. Ferromagnetic alignment has been observed in thin films first via magnetometry [65] where a small moment of $0.035 \mu_{B}$ 
per $\mathrm{Sm}^{3+}$ ion was found. This small moment brings with it a very large coercive field through the weak Zeeman interaction. X-ray magnetic circular dichroism (XMCD) measurements [52] have further characterised the residual moment in $\mathrm{SmN}$. This XMCD measurement was conducted at the $S m L_{2,3}$ edge to take advantage of the strong $d-f$ exchange present. Analysis of the results show the net moment is dominated by the orbital component. XMCD measurements on SmN / GdN super-lattices show the strong Gd-Sm exchange at the interface will rotate the Sm $4 f$ moment inline with that of the $\mathrm{GdN}$, and furthermore enhance the spin component of the magnetisation in $\mathrm{SmN}$ [19].

The unusual magnetic state of EuN has also seen some interest. In EuN Hund's rules again predict opposition between the spin and orbital components in the $4 f$ shell however it is now $J$ which is extinguished rather than $M$. This has been investigated experimentally $[27,66,67]$ were it was found that although $\mathrm{Eu}^{3+}$ will not order, the small concentration of nitrogen vacancies, essentially unavoidable to the order of $0.1 \%$, forms strongly magnetic $\mathrm{Eu}^{2+}$ ions. This concentration of $\mathrm{Eu}^{2+}$ then results in ferromagnetism in heavily doped films.

\subsection{Conclusions}

After a brief review of the theoretical and experimental literature of the rare earth nitrides, it is clear that although much progress has been made there remain areas where understanding is lacking, and there is great need for experimental input.

- The location of the lowest unoccupied $4 f$ bands in various members is still unclear.

With the current experimental interest in incorporating various lighter members of the series into devices $[11,17,68]$ and heterostructures [19, 20,18 ] the need to understand the transport properties, and thus the 
nature of the conduction band, is high.

The rare earth nitrides also offer the opportunity for studying the interplay of extended and localised states in a series of simply structured materials. Such a series would be a valuable addition to the family of strongly correlated electron systems. The true potential of the series, in this sense, can not be realised until the nature of the $4 f$ levels in the conduction and valence bands are determined.

This point is addressed in Chapters 4 and Chapters 5. The lowest unoccupied majority spin $4 f$ band is first shown to influence the transport channel in $\mathrm{SmN}$ and $\mathrm{NdN}$ via measurements of the anomalous Hall effect, discussed in Chapter 4 . These bands are then located via optical spectroscopy in Chapter 5.

- The effect of nitrogen vacancies on the electronic and magnetic properties of the rare earth nitrides remains unclear.

Any fabricated materials will always include some form of defect or vacancy, thus the effect of these must be understood. The problem is compounded as the highest quality films, in a structural sense, are grown at an elevated substrate temperature which leads to even higher vacancy concentrations than the $\sim 1 \%$ considered in the single band structure calculation which considers nitrogen deficient GdN. It is clear that vacancies on this level will have dramatic effects on the properties of the materials, the obvious example being the nature of the exchange in $\mathrm{GdN}$, which even being the simplest in the series, is far from understood.

This point is addressed in Chapter 6 where the properties of highly doped films are covered. Here structural, optical, electrical transport and magnetic measurements are all considered in the context of nitrogen vacancies. The observance of superconductivity in $\mathrm{SmN}$ is also discussed and shown to be present in only appropriately doped films. 


\section{Chapter 3}

\section{Film Growth and Characterisation}

This chapter covers the general experimental techniques used which are pertinent to the entire thesis. Specific techniques which are only applicable to a particular chapter are included in that chapter.

The present chapter begins by discussing the growth of rare earth nitrides and the specific systems used in this thesis. Capping layers are then considered, followed by substrates, growth geometry and masking. General experimental techniques are then discussed, beginning with Xray diffraction, electron microscopy and Dektak profilometry. During this discussion, two studies concerning the growth of capping layers are described. Finally, the techniques and equipment used for electrical transport and magnetic measurements are described.

\subsection{Film Growth}

Growth of the rare earth nitrides was first reported in the 1960s and since then various fabrication methods have been used. Many of the early techniques involved heating lanthanide metals in the presence of molecular nitrogen gas, however, other methods are reported including dissolving the lanthanide in liquid ammonia followed by distillation [9, 69]. The key difference between modern and historical methods is the use of ultra- 
high vacuum (UHV) systems. UHV systems can be used to more carefully control the growth environment, limiting the contamination from various sources, most notably atmospheric oxygen and water vapour [1]. Various UHV compatible growth methods are now used to fabricate rare earth nitride thin films such as; radio-frequency magnetron sputtering, pulsedlaser deposition, molecular beam epitaxy and physical vapour deposition. The final two of these techniques have been used to produce films for the present thesis.

Films were grown in two vacuum chambers on crystalline substrates mounted within. A Thermionics system with a base pressure of $\sim 1 \times 10^{-9} \mathrm{mb}$ was used to grow the majority of the films, a Riber Compact 21 system with a base pressure of $\sim 1 \times 10^{-10} \mathrm{mb}$ was also used.

The Thermionics system was equipped with a resistive heating source and an electron beam evaporator. Materials with sufficiently low evaporation temperatures could be evaporated in the resistive heating source such as metallic Sm, Dy and In. Sm, which evaporates at $\sim 500{ }^{\circ} \mathrm{C}$, was placed in a $\mathrm{BN}$ crucible and heated via current passed through a $\mathrm{W}$ filament. Dy, with a slightly higher evaporating temperature of $\sim 900{ }^{\circ} \mathrm{C}$, was heated using a $\mathrm{W}$ boat rather than the crucible, to increase thermal contact between the source and the material. For materials which require a higher temperature still (i.e. Gd, which evaporates at $\sim 1200^{\circ} \mathrm{C}$ ) the electron gun was used. Evaporation of materials in the Riber chamber was, in all cases, done with one of five effusion cells which were all electronically controlled.

Before nitrogen is introduced into the chamber, the lanthanide metal is heated to evaporation temperatures and used as a getter to further reduce the chamber pressure and provide a clean environment for growth. An example of the partial pressures inside the growth chamber as a function of time, prior to growth of a GdN film, are shown in Figure 3.1. Figure 3.1 shows the initial chamber partial pressures which first increases as the $\mathrm{Gd}$ is heated. Once the $\mathrm{Gd}$ reaches evaporating temperature the pres- 


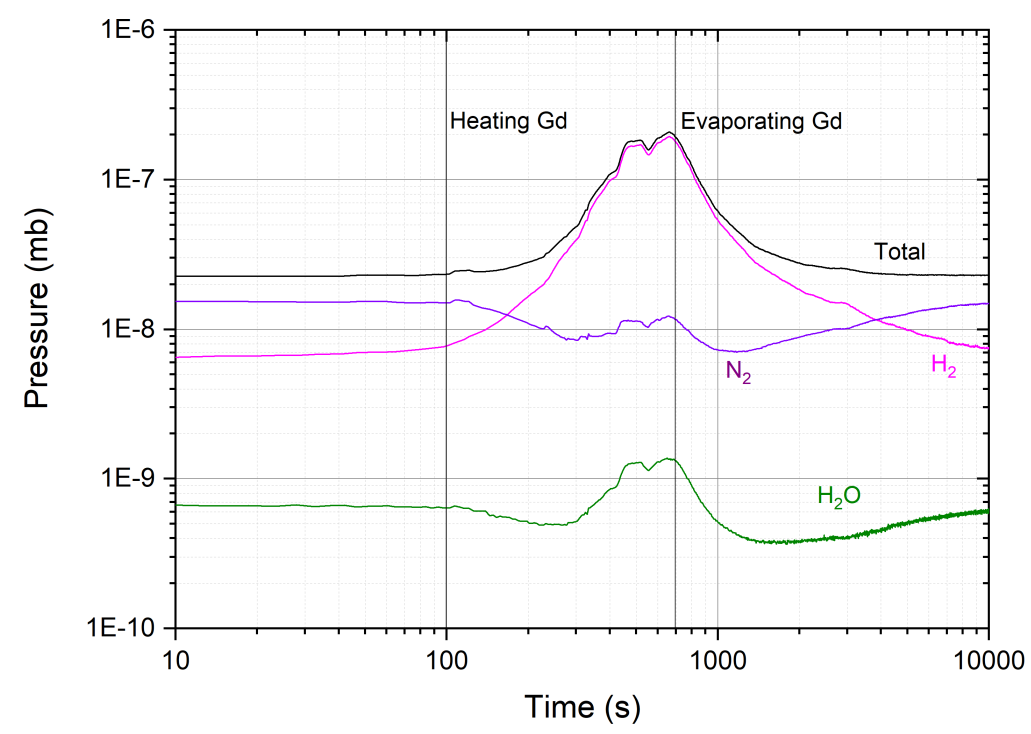

Figure 3.1: Plot of pressure as a function of time during the initial stages of growth of a GdN film (A612). The pressure initially increases as the Gd heats up along with various components in the chamber, once the $\mathrm{Gd}$ begins evaporating it acts as a getter decreasing the chamber pressure.

sure quickly drops as the Gd acts as a getter. Only a slight decrease in the partial pressure of $\mathrm{H}_{2} \mathrm{O}$ is seen before and after evaporation of $\mathrm{Gd}$, which indicates an initially clean chamber. The main contaminants in the chamber can be seen as $\mathrm{H}_{2}, \mathrm{~N}_{2}$ and $\mathrm{H}_{2} \mathrm{O}$. Hydrogen is a common contaminant in UHV systems as it will diffuse from grain boundaries in the stainless steel components in the chamber. At the level present in the system the $\mathrm{H}_{2}$ is not likely to cause any noticeable effect.

For the growth of a rare earth nitride, the stream of evaporated lanthanide was directed towards a substrate which, when appropriate, was rotated to ensure homogeneous deposition. The temperature of the substrate can be controlled during deposition using a heater mounted beneath the substrate holder in either system. Whenever possible substrates were out-gassed for at least one hour at $\sim 400 \mathrm{C}^{\circ}$ before deposition to ensure a clean surface. Whether out-gassing can be undertaken or not depends on 
the choice of substrate for a given growth (i.e. a substrate patterned with photoresist can not be heated at all while a substrate with $\mathrm{Cr} / \mathrm{Au}$ contacts can not be heated above $\sim 400 \mathrm{C}^{\circ}$

During evaporation of the lanthanide metal, ultra-high purity molecular nitrogen was directed towards the substrate. The flow rate was controlled using a mass flow controller. A typical rate was on the order of 1-4 standard cubic centimetres per minute, which when balanced by the pumping speed of the chamber resulted in a maintained pressure of $1-$ $40 \times 10^{-5} \mathrm{mb}$. Before entering the chamber nitrogen is passed through a Materson PUR-Gas in-line purifier to remove any contaminants. The partial pressures of various gasses in the chamber were monitored before and during the initial states of evaporation using a Pfeiffer Prisma massspectrometer. The ratio of $\mathrm{N}_{2}$ partial pressure to $\mathrm{H}_{2} \mathrm{O}+\mathrm{O}_{2}$ was maintained at more than $1 \times 10^{4}$ as contamination of these can significantly affect film quality.

For many of the lanthanides the clean metal layer, newly formed on the substrate, catalytically cracks the nitrogen bond directly forming the nitride [11]. The catalytic cracking of the nitrogen bond largely eliminates the need to activate the nitrogen for rare earth nitride growth. The deposition rate was monitored in real-time using a quartz microbalance and Lesker FTM-2400 digital rate monitor. The in-situ rate and thickness measurements are calibrated with $e x$-situ scanning electron microscopy (SEM), Dektak and reflectivity measurements. The careful control of the ratio of lanthanide to nitrogen flux is important as this is the factor which will, to a large extent, dictate the transport properties of a film.

\subsubsection{Capping}

The rare earth nitrides are known to react with $\mathrm{O}_{2}$ and $\mathrm{H}_{2} \mathrm{O}$ and decompose. Thus, for $e x$-situ measurements, a passivating capping layer must be grown on top of the finished film. There are various materials available 
for use as a capping layer such that the desired measurements of the film dictate the choice of capping material.

The most straightforward capping layers are made from the same lanthanide material that is used for the growth of the rare earth nitride. The procedure is that one simply shuts off the flow of nitrogen into the chamber once the desired film thickness is reached while continuing to evaporate the lanthanide metal. Although offering adequate protection this method is not desirable if magnetic measurements, optical measurements or in-plane transport measurements are required. For these reasons insulating, transparent and non-magnetic capping layers such as AlN or GaN are typically used. The growth of AlN or GaN requires molecular nitrogen to be activated. In the Thermionic system this is done using a Kaufman ion source and in the Riber Compact 21 a radio frequency plasma source. The growth details of these materials are not discussed here; however, the use of the radio frequency plasma source in the Riber system created difficulties in the growth of the rare earth nitride layers. This was investigated and thus, is briefly discussed.

\section{Further Investigations Into Capping Layers Involving N Plasma}

Rare earth nitride samples were grown successfully in the Riber system with capping layers that did not require activated nitrogen or a nitrogen plasma (i.e. $\mathrm{Sm}, \mathrm{Gd}, \mathrm{Al}$ and $\mathrm{Ge}$ ). The quality of the rare earth nitride films in question was verified primarily via $x$-ray diffraction (XRD) and transport measurements. When capping layers that required the use of a nitrogen plasma were grown on top of the film, the rare earth nitride layers were significantly affected by the exposure to the nitrogen plasma. For rare earth nitrides capped using the nitrogen plasma the characteristic rare earth nitride peaks vanished from the XRD signal and transport measurements found a significantly enhanced resistance. An example of this is shown in Figure 3.2 in which the left-hand panel shows an XRD $2 \theta$ plot of a SmN sample capped with $\sim 300 \mathrm{~nm}$ of Ge in black and a SmN sample, 

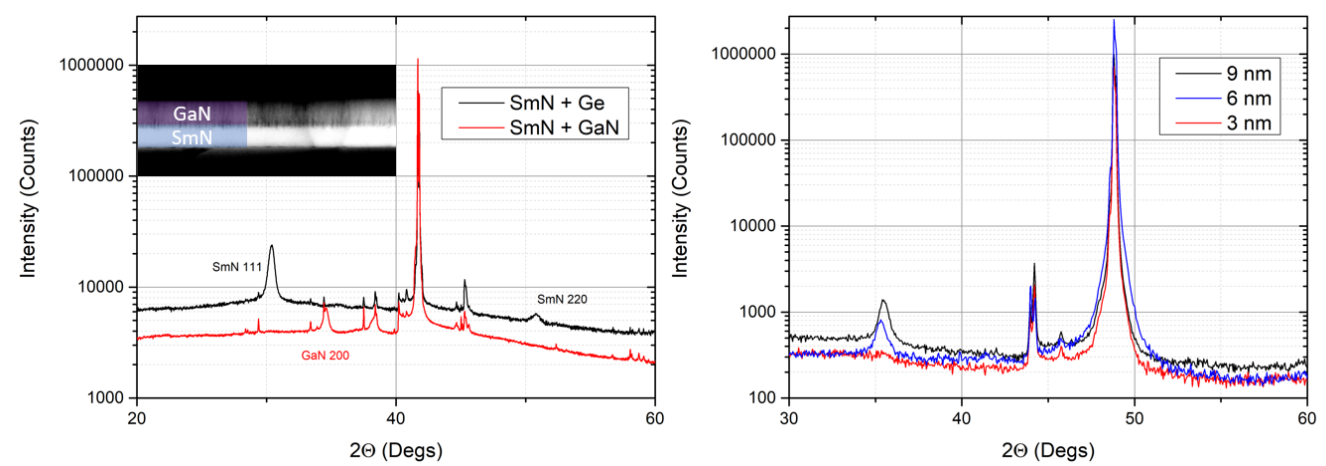

Figure 3.2: Left: $\mathrm{XRD} 2 \theta$ plots of a $\mathrm{SmN}$ film capped with Ge (black) and a SmN film capped with $\mathrm{GaN}$ (red). The GaN capped film loses all characteristic rare earth nitride peaks. The inset shows an SEM image of the GaN capped film. Right: XRD $2 \theta$ plots of three SmN films capped with AlN with $3 \mathrm{~nm}, 6 \mathrm{~nm}$ and $9 \mathrm{~nm}$ of $\mathrm{Al}$ grown between the $\mathrm{SmN}$ and AlN layers. The Al buffer layers protect the $\mathrm{SmN}$ from the effects of the nitrogen plasma for the $6 \mathrm{~nm}$ and $9 \mathrm{~nm}$ thick buffer layers.

grown under comparable conditions, capped with $\sim 300 \mathrm{~nm}$ of $\mathrm{GaN}$ in red. The SmN 111 peak is visible in the black Ge capped series but absent from the red GaN capped series. The inset shows an SEM image of the GaN capped sample where the SmN layer is identified. Transport measurements were conducted on both films with the Ge capped film displaying a result typical for $\mathrm{SmN}$ (not shown) while transport measurements on the GaN capped film again found a significantly enhanced resistance. Note that this large resistance is not simply the contact resistance of the GaN layer but in the SmN layer itself. To determine if the effect on the film was being caused by the GaN itself rather than the plasma a SmN film was grown, exposed to the plasma for several minutes then capped with Ge. This film suffered the same lack of an XRD signal, and large resistance as the GaN capped film, indicating the impinging $N$ plasma causes the effect rather than the GaN layer.

To investigate further, a series of $\mathrm{SmN}$ films was grown using $\mathrm{AlN}$ as a 
capping layer. The initial deposition of the capping layer was conducted with the nitrogen plasma directed away from the sample, the goal being to deposit a protective layer of $\mathrm{Al}$ before exposing the sample to the nitrogen plasma. Films with Al buffer layers of $3 \mathrm{~nm}, 6 \mathrm{~nm}$ and $9 \mathrm{~nm}$ were grown. XRD $2 \theta$ measurements for these films are shown in the right-hand panel of Figure 3.2. The right-hand panel of Figure 3.2 shows the SmN 111 peak is visible for the sample with the $9 \mathrm{~nm}$ and $6 \mathrm{~nm}$ buffer layers but absent in the sample with the $3 \mathrm{~nm}$ buffer layer. It appears that less than $6 \mathrm{~nm}$ of $\mathrm{Al}$ will not adequately protect the film from the damaging effects of the nitrogen plasma.

The degradation of rare earth nitride films, when exposed to nitrogen plasma, is both surprising and unexplained. Similar results were not found when exposing films to the Kauffman ion source in the Thermionics system. Ultimately no explanation is offered for this observation at present. It is clear a more systematic study of the effects of the nitrogen plasma is required.

\subsubsection{Substrate Selection, Film Geometry and Masking}

The choice of substrate, capping layer and geometry for each sample was determined by the measurement required. The majority of films required multiple measurements, so a film was simultaneously grown on a range of substrates suitable for each measurement, this is summarised in Table 3.1. Electrical measurements were conducted on sapphire substrates as the material is insulating and non-magnetic. The crystalline structure of sapphire also makes it possible to cleave regularly sized pieces for use in masks. XRD measurements were conducted on sapphire as it is largely featureless between $2 \theta=20^{\circ}$ to $2 \theta=40^{\circ}$ where the major rare earth nitride peaks are located. SEM measurements were conducted on P- or As-doped $\mathrm{Si}$ as conductive substrates are required to make electrical contact with the SEM stub. If samples were grown at high-temperature P-doped Si topped 


\begin{tabular}{|c|c|c|c|c|c|c|}
\hline & XRD & SEM & SQUID & Electrical & UV-VIS & IR \\
\hline $\mathrm{Al}_{2} \mathrm{O}_{3}$ & $\checkmark$ & & & $\checkmark$ & $\checkmark$ & \\
\hline Doped $\mathrm{Si}$ & & $\checkmark$ & & & & \\
\hline Undoped $\mathrm{Si}$ & & & $\checkmark$ & & & $\checkmark$ \\
\hline
\end{tabular}

Table 3.1: Table of substrate materials and measurements

with $300 \mathrm{~nm}$ of $\mathrm{SiO}_{2}$ or AlN buffered $\mathrm{Si}$ was used to prevent the formation of a silicide at the lanthanide / Si interface. Magnetic measurements were typically conducted on undoped Si substrates. For optical spectroscopy, high energy measurements in the near infra-red region and above were conducted on sapphire as the material is largely transparent here. Low energy measurements from the mid infra-red and below were performed on un-doped $\mathrm{Si}$ as this is largely transparent at low energy. In general, all measurements would be conducted on a separate substrate, so six different substrates were loaded simultaneously. Films were generally capped with AlN as it was compatible with all the above measurements.

\section{Masking - Top Contacts in van der Pauw Geometry}

Although in some cases it is possible to conduct a four-point resistance measurement through the capping layer, by attaching wires directly with silver paint, this is a somewhat unreliable method. The large two-point resistance between any two contacts caused by the capping layer itself can cause difficulties and introduce unnecessary noise into the measurement. To make more reliable electrical contact and reduce two-point resistance $\mathrm{Cr} / \mathrm{Au}$ contacts were evaporated onto the surface of the capping layer using an Angstrom Engineering evaporator. This reduced two-point resistance by up to an order of magnitude in most cases. A schematic for the mask used when evaporating the $\mathrm{Cr} / \mathrm{Au}$ contacts can be seen in the lefthand panel of Figure 3.3. The mask is machined from $1 \mathrm{~mm}$ thick stainless steel with slots recessed $0.5 \mathrm{~mm}$. The four slots offer either $10 \times 10 \mathrm{~mm}^{2}$ 

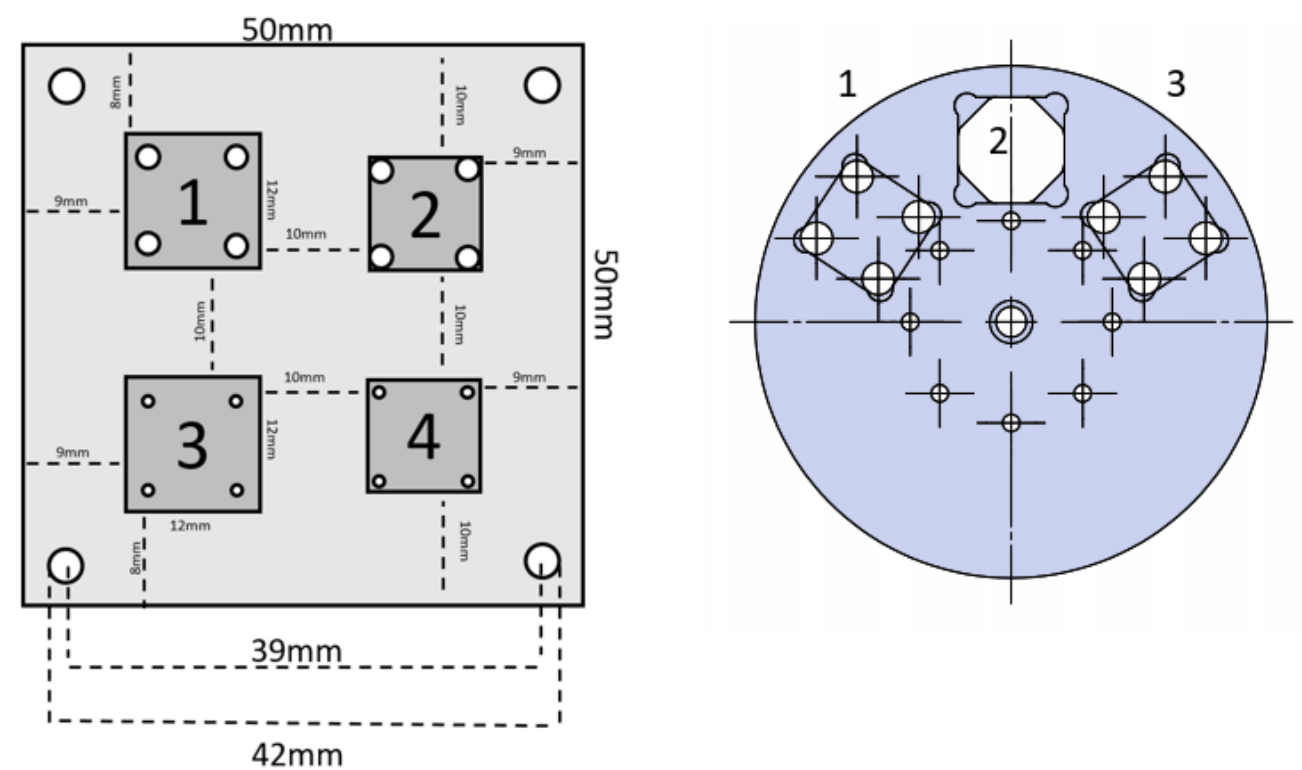

Figure 3.3: Left: Schematic of mask used to evaporate Cr / Au contacts on top of a finished film. Right: Schematic of a Mo mask used for depositing bottom contacts and high-temperature growth.

$(2,4)$ or $12 \times 12 \mathrm{~mm}^{2}(1,3)$ recesses into which substrates may be placed. There is then the additional choice of contact size of $1 \mathrm{~mm}$ diameter $(3,4)$ or $2 \mathrm{~mm}$ diameter $(1,2)$.

\section{Masking - Bottom Contacts in van der Pauw Geometry}

The above method of top contacting via $\mathrm{Cr} / \mathrm{Au}$ pads was relatively successful, though, the two-point resistance from the capping layer still caused problems, in particular in low-temperature measurements. To improve the situation, a method of bottom contacting films was developed using the mask visible in the right-hand panel of Figure 3.3. The mask in the right-hand panel of Figure 3.3 is machined from $1 \mathrm{~mm}$ thick Mo with slots 1,2 and 3 all $10.5 \times 10.5 \mathrm{~mm}^{2}$ recessed $0.5 \mathrm{~mm}$. The material for the mask was chosen for its high melting point to allow the growth of samples at an elevated temperature while mounted in the mask. 
To begin, clean substrates were inserted into slots 1 or 3 and contacting material deposited. The mask and substrates were then removed from the system, and the bottom contacted substrate loaded into slot 2 , the rare earth nitride film and capping layer can then be deposited. The initial rare earth nitride deposition will fall on the inner edges of the contacts; this is then covered with the capping layer while the outer edges of the contacts, hidden by the mask, remain exposed. Once removed from the system wires can be connected to contacts at the edge of the sample. These contacts are now in direct electrical contact to the rare earth nitride. The reduced two-point resistance, due to bypassing the capping layer, reduces noise and greatly simplifies measurements.

The mask also features $2 \mathrm{M} \times 2$ tapped holes around the centre for attaching additional substrates with Mo screws. The central hole is a $3 \mathrm{~mm}$ diameter circle within a recessed circle of $3 \sqrt{2} \mathrm{~mm}$ such that a $3 \times 3 \mathrm{~mm}^{2}$ square substrate can be loaded and a $3 \mathrm{~mm}$ diameter deposition made in the centre.

\subsection{Structural Characterisation}

\subsubsection{X-Ray Diffraction}

XRD measurements were conducted primarily with a PAN-alytical X'Pert PRO equipped a $\mathrm{Cu} X$-ray source. Several measurements were also performed on a Boman D8 with a Co source.

The basic principle of structural characterisation via XRD is to take advantage of Bragg diffraction [70] which is caused by the specular reflection of X-rays of wavelength $\lambda$ from lattice planes in crystalline solids, separated by some distance $d$. When light reflection from adjacent planes is constructive the path difference between the planes, which is dependent on the incident angle $\theta$, is equal to some integer multiple $n$ of the wavelength of the light. This can be described by the Bragg equation 


$$
2 d \sin (\theta)=n \lambda .
$$

The expected $d$ spacing between adjacent planes, which depends on the lattice parameter $a$, is well known for different crystal structures. By measuring the intensity of reflected light as a function of the angle $\theta$ and noting the locations of peaks in the spectrum, which indicate points of constructive interference, it is possible to determine both the crystal structure of a material and the out of plane lattice parameter $a$. When multiple orders of peaks are resolved it is possible to extend this analysis using the method of Nelson and Riley [71].

The width $\beta$ of a given peak can be shown to be proportional to the average size of the crystallites $\tau$ in a sample [72]

$$
\tau=\frac{K \lambda}{\beta \cos (\theta)}
$$

where $K$ is a dimensionless constant determined by the shape of the crystallites. In this thesis, $K$ is taken as equal to 1 .

Any misalignment of a sample on the measurement stage will cause a shift in the resulting spectrum, which was accounted for up to some linear offset $\theta_{c}$ as follows. The first and second order reflections of a given peak must physically return the same lattice parameter. For the case of the commercially produced substrates, this lattice parameter should furthermore be very close to the literature value. With this in mind, all measurements were conducted over a range such that the first and second order substrate peaks were fully resolved, which was generally the sapphire 0006 and 00012 reflections. It was then assumed that, to first order, there was some offset in the measured spectrum $\theta_{c}$ such that the following sum could be minimised for some value of $\theta_{c}$,

$$
a_{1}\left(\theta+\theta_{c}\right)+a_{2}\left(\theta+\theta_{c}\right)-2 a_{l i t}=0,
$$

where $a_{1}$ and $a_{2}$ are the experimental lattice parameters derived from the 

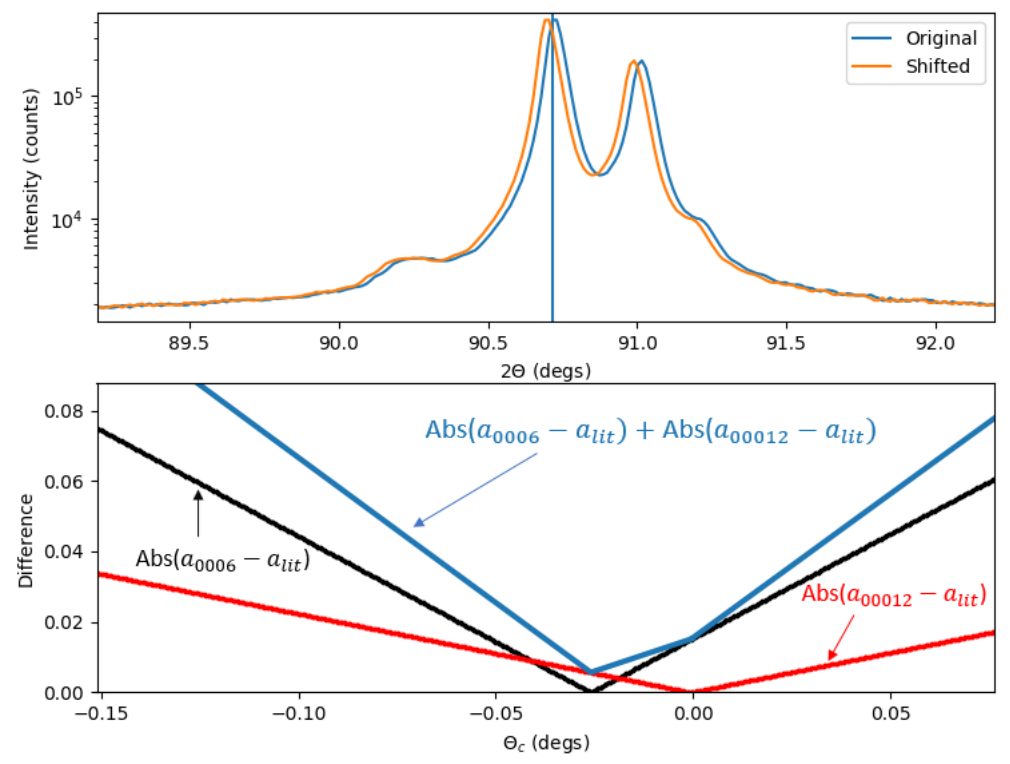

Figure 3.4: Top: Example of the correction of an XRD measurement for misalignment, the original spectrum is shown in blue, and the shifted in orange. Bottom: An offset is added to the spectra to minimise the difference between the lattice parameter from the first and second order substrate peaks and the literature value. The difference between the sum for the first and second order lattice parameters and the literature value is shown in blue as a function of the offset value $\theta_{c}$. The minimum of the blue curve represents the calculated value of $\theta_{c}$

first and second order reflections respectively and $a_{l i t}$ is the literature value for the lattice parameter. This process was completed for all measurements; an example is shown in Figure 3.4. The top panel of Figure 3.4 shows the original spectrum in blue and the shifted spectrum in orange and is centred on the sapphire 00012 peak. The bottom panel shows $a_{1}-a_{l i t}$ in black, $a_{2}-a_{l i t}$ in red and the sum of these in blue as functions of $\theta_{c}$, the $\mathrm{x}$-axis value at the minimum of the blue curve is the value of $\theta_{c}$ used to correct the experimental measurement.

Once a spectrum had been shifted by some value $\theta_{c}$ the relevant rare earth nitride peaks were fitted using the peak analysis software included in Origin 9.1 Pro. Peaks were generally fitted using a single Lorentzian. 


\section{Further Investigations into Oxygen Contamination}

It is apparent that oxygen contamination increases the resistivity of a film and may also have an effect on the lattice parameter [73]. To investigate this, both the lattice parameter and resistance of a sample were measured as a function of time while the sample was exposed to atmosphere.

To conduct the experiment a four-point resistance data logger was constructed using a Lakeshore 101 current source which applies $10 \mu \mathrm{A}$ with a maximum voltage of $5 \mathrm{~V}$ and an Arduino equipped with an expanded voltage sensor an 8 bit ADC converter running on custom software. Electrical contact was made to the sample by attaching $0.05 \mathrm{~mm}$ diameter $\mathrm{Cu}$ wires, coated with $2 \mu \mathrm{m}$ polyestermind insulation, to the capping layer directly with silver paint. The experiment was conducted on a SmN sample capped with Ge as a Ge cap was found to allow oxygen contamination over the time scale of hours.

The top left-hand panel of Figure 3.5 shows the XRD $2 \theta$ scans over the SmN 111 peak, time progress from the dark red to yellow scans. As time progresses the centre of the peak shifts to lower angles, indicating an increase in lattice parameter. The amplitude of the peak also decreases as time passes. The centre of each peak was used to determine the lattice parameter of the sample, which is plotted as a function of time in the top right-hand panel of Figure 3.5. The lattice parameter increases as time passes by $\sim 0.4 \%$. The increase is relatively steady until roughly $80 \mathrm{~min}-$ utes at which point the lattice parameter increases rapidly until measurement was no longer possible. The lower left-hand panel shows the resistance as a function of time. Here we see the sample resistance initially rising rather slowly; it has increased by a factor of two when it reaches a peak at $\sim 80$ minutes. There is then a small decrease followed by a very fast increase in resistance before the measurement limit was reached. Finally, the bottom right-hand panel shows the sample resistance as a function of lattice parameter. Here we initially see a positive correlation until the resistance reaches $\sim 16500 \Omega$ at which point the lattice continues to 

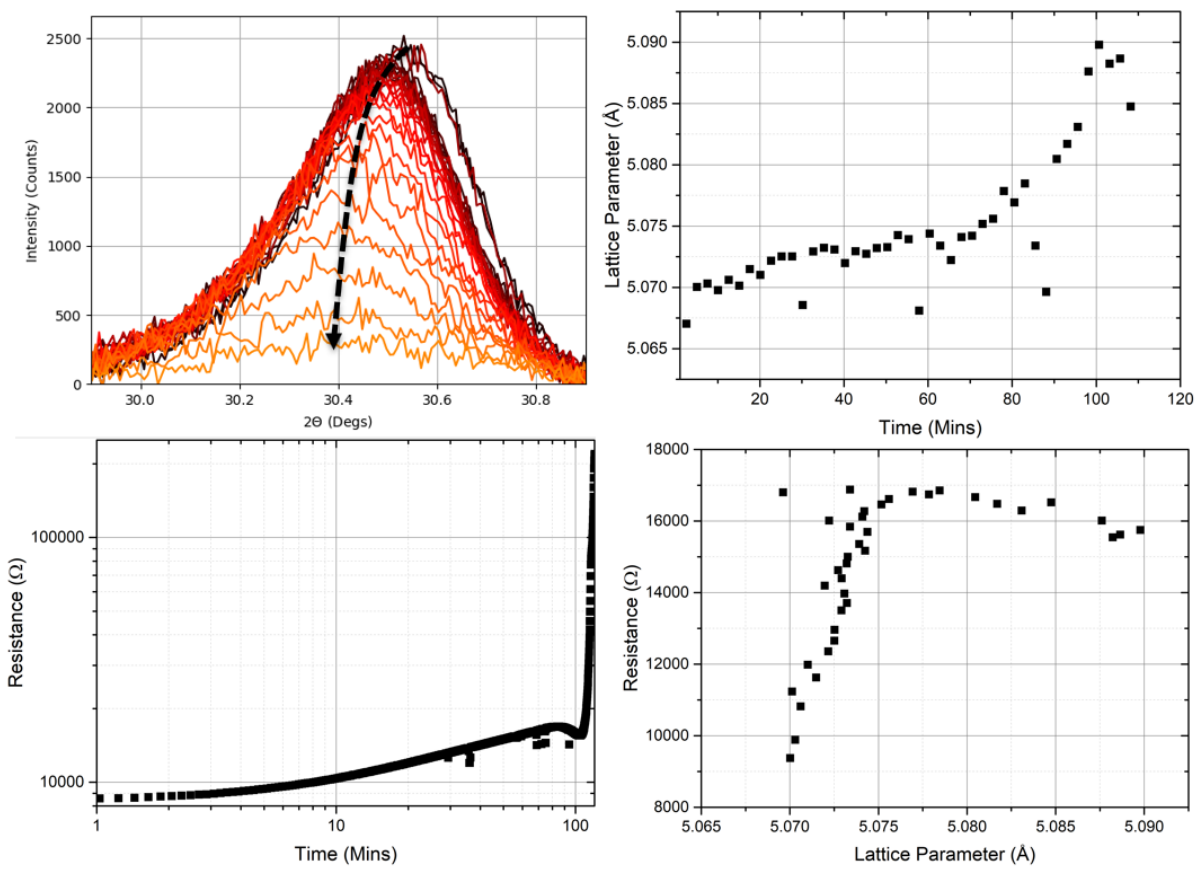

Figure 3.5: Measurements undertaken on SmN sample capped with Ge (R039). Top left: XRD $2 \theta$ scans of the SmN 111 peak taken over roughly two hours. Top right: Lattice parameter as a function of time calculated from the measurements in the top left panel. Bottom left: Measurements of resistance as a function of time. Bottom right: Resistance and a function of lattice parameter.

expand while the resistance shows a slight decrease. It appears that from the moment the film is exposed to oxygen, both the lattice parameter and resistance are affected. The initial positive correlation in the lower righthand panel of Figure 3.5 shows the two parameters are likely related.

The goal of this experiment was to determine the effect of oxygen contamination on the lattice parameter of $\mathrm{SmN}$ samples. Measurements shown in Figure 3.5 indicate that as films are exposed to oxygen and water vapour, the lattice will expand, and the resistance will increase. The expansion seen during the experiment of $\sim 0.4 \%$ is not insignificant. These results also show that the effects of oxygen contamination may be apparent in other measurable properties (i.e. lattice parameter) before they are appar- 
ent, in the sense of a dramatically increased resistance. Further experiments were carried out on films with GaN capping layers of which one (film A433) grown in the Thermionics system is briefly discussed. Measurements of the lattice parameter as a function of time were conducted as above. XRD scans were taken over the SmN 111 peak for $\sim 20$ hours with no measurable change in the lattice parameter. This shows that reliable capping layers can be constructed which do not allow oxygen to diffuse through.

\subsubsection{SEM and Dektak}

Ex-situ sample thickness was measured by SEM, which enabled the thickness of individual layers to be determined, and Dektak profilometry, which measured the total sample thickness. Measurement via SEM requires good electrical contact between the sample and SEM stub to avoid charging from the measurement tip. For this reason, conductive $\mathrm{Si}$ substrates were included in all growths for use in SEM measurement. Profilometry measurements were much faster and could be undertaken on any substrate. Profilometry was generally conducted on multiple substrates included in the growth, including the substrate used for SEM measurements.

To measure the profile of a sample in the SEM the sample had to be cleaved exposing the edge of the rare earth nitride layer to atmosphere. This exposure to atmosphere can cause the sample edge to expand, resulting in a larger apparent thickness in the SEM image for the rare earth nitride layer. The capping layers are not sensitive to atmosphere, so their thickness measured via SEM should be accurate. Dektak measurements were of the total sample profile, so do not require exposing the unprotected edge of the sample to atmosphere.

An example of each technique is shown in Figure 3.6. Figure 3.6 shows an SEM image on the left and a Dektak measurement on the right, each measurement is taken from the same piece of the sample. In the left- 

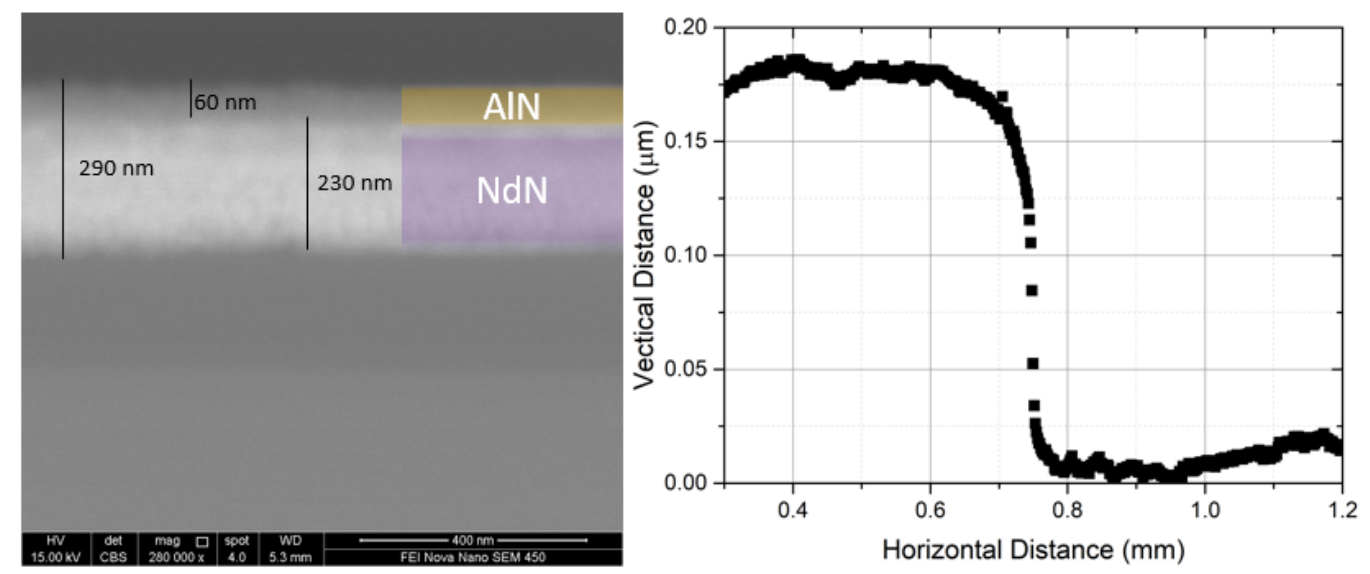

Figure 3.6: Left: SEM image of the cross section of a NdN sample capped with AlN, the total thickness here is $\sim 290 \mathrm{~nm}$. Right: Dektak measurement on the same sample shown in the left hand panel with a total thickness of $\sim 180 \mathrm{~nm}$.

hand image, the $\mathrm{NdN}$ layer is identified as the lighter layer measuring $\sim 230 \mathrm{~nm}$, the AlN capping layer is $\sim 60 \mathrm{~nm}$. Although from the image it is difficult to tell the exact layer thickness it is clear that the entire stack is $\sim 290 \mathrm{~nm}$, which contrasts the Dektak measurement on the right with a total thickness of $\sim 180 \mathrm{~nm}$. It seems that here in the sample measured via SEM, the unprotected NdN edge has expanded as it is exposed to atmosphere. In such a case where there is significant disagreement between SEM and Dektak measurements, further measurements would be made to determine the film thickness, these were usually reflectivity (X-ray or optical). Reflectivity measurements in most cases were in-line with the Dektak measurements indicating that exposure of the edge of the film to atmosphere can indeed result in an enhanced thickness for SEM measurements. 


\subsection{Electrical Measurements}

\section{Janis Close Cycle Cryostat}

A Janis SHI- $4 \mathrm{H}$ close cycle He cryostat was used for measurements of resistance as a function of temperature from $300 \mathrm{~K}$ to $\sim 4 \mathrm{~K}$. In this cryostat samples were mounted on a copper cold finger using a small amount of Apiezon $\mathrm{N}$ grease to hold the sample in place while making good thermal contact. Electrical connections were made using silver paint to attach copper wires to contact locations, which were typically $\mathrm{Cr} / \mathrm{Au}$ pads in a van der Pauw Geometry discussed in section 3.1.2. Measurements of the two-point resistance between any two contacts were recorded to confirm good electrical contact had been made. Four-point resistance was then measured as a function of temperature, using an appropriate current, which was typically $0.1 \mu \mathrm{A}$ to $100 \mu \mathrm{A}$, chosen with the two-point sample resistance in mind.

The temperature was controlled using a Lakeshore 340 temperature controller and an RDK coldhead. Measurement electronics consisted of a Keithley 224 current source, a Keithley 182 voltmeter and a Keithley 196 multimeter. Control of all electronics and temperature was automated via Labview. The Keithley 224 current source was able to apply up to $105 \mathrm{~V}$ at a minimum of $0.01 \mu \mathrm{A}$ so could push a current through a resistance of up to $\sim 105 \times 10^{8} \Omega$. The Keithley 182 voltmeter could, however, measure a maximum of $3 \mathrm{~V}$ limiting the system to a maximum sample resistance of $3 \times 10^{8} \Omega$.

\section{Quantum Design Physical Properties Measurement System}

A Quantum Design Physical Properties Measurement System (PPMS) is a device designed for measuring various physical properties, including electrical, magnetic and thermal of which electrical measurements were the most common. The PPMS is capable of measurements at temperatures from $1.9 \mathrm{~K}$ to $300 \mathrm{~K}$ and in a magnetic field at up to $\pm 9 \mathrm{~T}$. For all electrical 
measurements samples were mounted inside a rotating stage allowing the orientation of the sample to be changed with respect to the magnetic field direction between 0 and $360^{\circ}$ along one axis.

Measurements were typically conducted by mounting samples on commercial pucks, of which two types were used. On A typical PPMS puck, there is $\mathrm{a} \sim 10 \times 10 \mathrm{~mm}^{2}$ area for the sample to be mounted and eight electrical contacts. Samples were mounted on the puck with a small amount of Apiezon N grease or GE Varnish to keep the sample in place and provide good thermal contact. Electrical contact was made between the puck and sample using $0.05 \mathrm{~mm}$ diameter $\mathrm{Cu}$ wire attached to sample measurement locations with silver paint and to the puck with solder. The two-point resistance between any two points was then measured to confirm good electrical contact.

The second type of puck used was a Wimbush Science and Technology spring-loaded press contact puck. These consist of a base similar to the standard PPMS puck with an additional cap equipped with spring-loaded $\mathrm{BeCu}$ Au plated pins with a $1.5 \mathrm{~mm}$ travel, attached to the base with $\mathrm{BeCu}$ screws. Pins were located in a square fashion spaced each by $8 \mathrm{~mm}$ such that they would align with either the $\mathrm{Cr} / \mathrm{Au}$ pre-deposited or top contacts discussed in Section 3.1.2. The ease of use, superior electrical contact and reduced exposure to atmosphere made the combination of pre-deposited contacts and the Wimbush Science and Technology spring-loaded press contact puck the preferred combination of contact geometry and electrical measurement.

Electrical measurement in the PPMS was limited to a maximum measured voltage of $95 \mathrm{mV}$, and in theory, the two-point resistance of a sample could be between $4 \mu \Omega$ and $1 \mathrm{M} \Omega$. 


\subsubsection{Van der Pauw Geometry}

Resistance measurements were typically conducted in a van der Pauw geometry as discussed in Section 3.1.2. When using the van der Pauw method, the following conditions must be met

- The sample must have a uniform thickness

- The sample must have a simple geometry

- Electrical contacts must be small and at the periphery of the sample

Furthermore, when measured in this geometry one must be aware that the measured resistance is often different when measured along different directions, i.e. for a measurement from four points A, B, C, D located at the corners of square $R_{A B C D} \neq R_{B C A D}$ where $R_{A B C D}$ and $R_{B C A D}$ are defined in Figure 3.7. It can be shown that the following relationship holds between the measured resistances $R_{A B C D}, R_{B C A D}$ and the sheet resistance $R_{s}$ [74],

$$
1=\exp \left(-\pi \frac{R_{A B C D}}{R_{s}}\right)+\exp \left(-\pi \frac{R_{B C A D}}{R_{s}}\right) .
$$

The above equation can be solved analytically for the case that $R_{A B C D}=$ $R_{B C A D}=R$ in which case $R_{s}=\pi / \ln (2) R$ but in all other cases must be solved numerically. Figure 3.7 shows a plot of the above equation as a function of $R_{s}$ for values of $R_{A B C D}=100 \Omega, R_{B C A D}=200 \Omega$ The value of $R_{s}$ corresponding to a value of the function closest to one represents the closest to the sheet resistance of the sample, in this case $\sim 653 \Omega$. This numerical process was used when determining the sheet resistance of all samples when measurement of both $R_{A B C D}$ and $R_{B C A D}$ was possible.

\section{Hall effect measurements}

Hall effect measurements, above the magnetic transition temperature, were conducted on samples using a van der Pauw geometry in the Quantum Design PPMS system described above. In a completely homogeneous 


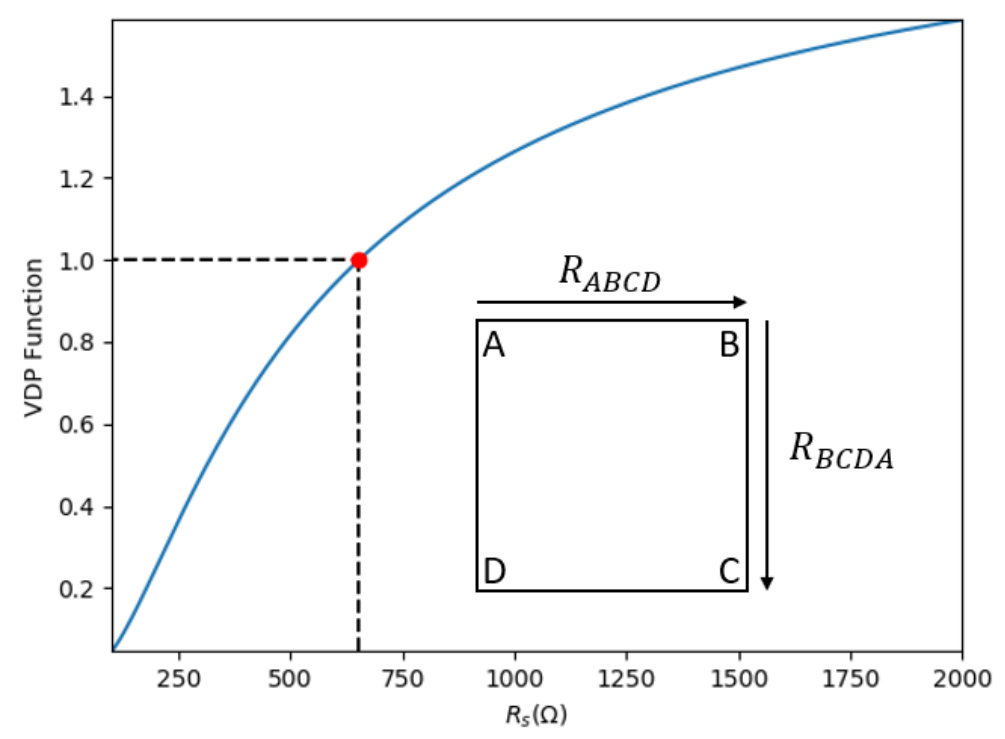

Figure 3.7: Plot of the function shown in Equation 3.1 for values of $R_{A B C D}=$ $100 \Omega, R_{B C A D}=200 \Omega$ The value of $R_{s}$ corresponding to a value of the function closest to one represents the closest to the sheet resistance of the sample, in this case $\sim 653 \Omega$.

sample with perfectly aligned contacts Hall effect measurements in the van der Pauw geometry would result in a completely current transverse voltage $V_{x y}$. This is, however, never the case so one must adjust the measurement for the parasitic longitudinal voltage $V_{x x}$. The general procedure used for measuring the Hall effect and separating the transverse from the longitudinal component is as follows.

Samples were first held at the appropriate measurement temperature for $\sim 5$ minutes to minimise the effects of temperature drift during the measurement. The magnetic field was then swept to the required value and the first set of measurements taken. The sample was then rotated $180^{\circ}$ and the measurement repeated, this rotation has the effect of reversing the orientation of the magnetic field. As the transverse Hall voltage is odd in applied field while the parasitic longitudinal component is even, a subtraction is now possible. Measurements at each field location are 


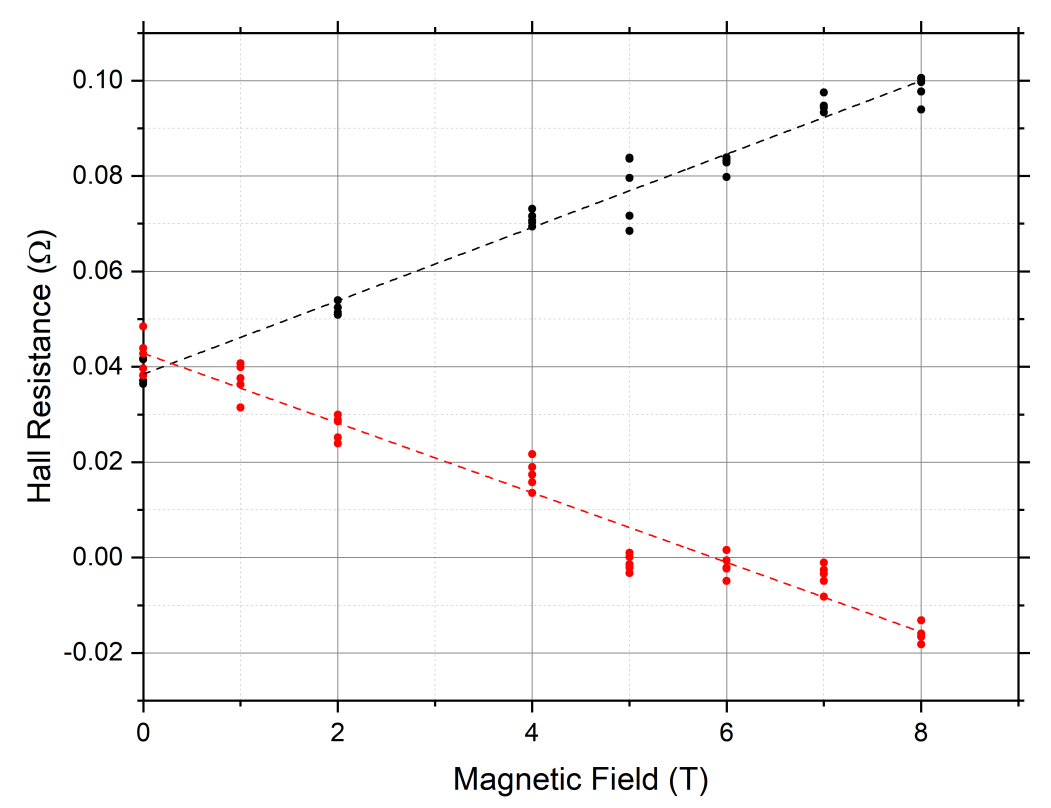

Figure 3.8: Plot of the Hall resistance as a function of applied field in a SmN sample (A605), the red and black series show the sample in differing orientations with respect to the applied field.

repeated five times, in each orientation, as the field is swept up. The whole series of measurements is then repeated as the field is swept down.

Samples were measured between $0 \mathrm{~T}$ and $8 \mathrm{~T}$ in $1 \mathrm{~T}$ increments. An example of the raw measurement is below in Figure 3.8, which shows the measurements in both orientations as a function of applied field. The gradient taken from a linear fit to the data can be used to determine the carrier concentration of the sample, as discussed in Section 2.2.1.

\subsection{Magnetic Measurements}

Magnetic measurements were conducted in a Quantum Design Magnetic Properties Measurement System (MPMS). The MPMS, or superconducting quantum interference device (SQUID), is centred around two Josephson junctions connected into a superconducting loop and uses the DC Joseph- 
son effect to determine the magnetic field of any material placed in the loop. In short, the working principle is as follows. If a magnetic material is placed into the superconducting loop, an opposing field will be generated via an induced current in the loop. This induced current now creates an additional Josephson phase in the junctions, which is proportional to the number of magnetic flux quanta passing through the loop. The voltage over these junctions will now oscillate and can be counted to determine the magnetic moment of the material placed inside the loop.

Magnetic measurements were conducted on samples grown on undoped Si substrates as this is largely non-magnetic. Samples were cut to the appropriate size $\sim 5 \mathrm{~mm} \times 10 \mathrm{~mm}$ and inserted into a plastic straw, which is the standard procedure for mounting samples in the system.

Typically two types of magnetic measurements are routinely undertaken on the rare earth nitrides. This is, (i) the measurement of the magnetic moment as a function of temperature at a constant, or zero applied field, and (ii) measurement of the magnetic moment as a function of applied field at a constant temperature. Above the Curie temperature, the rare earth nitrides exist in the paramagnetic phase and are generally well described by the Curie-Weiss law, which describes the magnetic susceptibility of a material as

$$
\chi=n \mu_{B}^{2} g_{J}^{2} \frac{J(J+1)}{3 k_{b} T},
$$

where $n$ is the concentration of magnetic ions, $K_{b}$ is the Boltzmann constant, $T$ is the temperature and $g_{j}$ is the Landé $g$ factor. The effective paramagnetic moment can be determined from the susceptibility as $m_{\text {eff }}=$ $g_{j} \sqrt{J(J+1)} \mu_{B}$. 


\section{Chapter 4}

\section{The Anomalous Hall Effect in Rare Earth Nitrides}

This chapter describes measurements of the anomalous Hall effect on various rare earth nitrides. To start, a background of the anomalous Hall effect is given beginning in a historical context and moving on to the modern Berry phase description. A semi-classic description is then given which is used throughout the remainder of the chapter. Following this a motivation of the study of the anomalous Hall effects in the rare earth nitrides is given, then the specific experimental techniques used are described. Finally experimental results on $\mathrm{SmN}, \mathrm{NdN}$ and $\mathrm{GdN}$ are discussed.

\subsection{Theoretical Background}

One year after his discovery of the ordinary Hall effect [75] Hall himself recorded an enhanced Hall resistance in ferromagnetic iron [76]. This anomalous contribution saturated at some point proportional to the sample magnetisation [77], thus the empirical relationship for the transverse resistivity

$$
\rho_{x y}=R_{0}(n) B+R_{s}(n, \tau) M
$$


was established, where $R_{0}(n)$ is the coefficient of the ordinary Hall effect and $R_{s}(n, \tau)$ is the empirical coefficient for the anomalous Hall effect, both of which depend on intrinsic properties of the material. The coefficient of the ordinary Hall effect has the simple proportionality of $R_{0}(n) \propto 1 / n$, as discussed in Section 2.2.1.

The anomalous Hall effect is now considered to be comprised of three distinct contributions, two extrinsic disorder related contributions, skew $[78,79]$ and side-jump [80] scattering, and one intrinsic contribution [81, 82], with all three caused by the spin-orbit interaction. The skew scattering contribution is caused by scattering due to spin-orbit coupling between a conduction electron and an impurit,y which will deflect the incident electron along a new trajectory. The side-jump is a similar displacement but now transverse to the direction of motion. These two impurity related scattering effects are generally superimposed such that an electron scattered off an impurity will find a new velocity vector via skew scattering, this new velocity vector will not intersect the previous at the scattering centre due to the side jump [80]. The intrinsic contribution is caused by the spin-orbit interaction between the itinerant conduction electron and the host ions of the lattice.

In most modern treatments of the anomalous Hall effect the intrinsic contribution is described in terms of a geometric Berry phase [83] of the conduction electrons, rather than the original semi-classical description [81]. A brief description of this modern treatment is now given based on a recent review by Xiao et. al [84] and a description given by Ong and Lee [85]. This is followed by a semi-classical description which is more practical in terms of interpreting the present experimental results [12].

In general the Berry phase can be described by considering a Schrödinger equation

$$
H(\boldsymbol{R})|n(\boldsymbol{R})\rangle=\epsilon_{n}|n(\boldsymbol{R})\rangle
$$

with $\boldsymbol{R}=\boldsymbol{R}(t)\rangle$. As given by the quantum adiabatic theorem, a system 
initially in an eigenstate $\mid n(\boldsymbol{R}(t=0)\rangle$ will remain in the eigenstate as it is smoothly and slowly (i.e. $T_{E} \gg T$ where $T_{E}$ is the external time period of the transportation and $T$ is the time period of the Hamiltonian) transported along a path $C$. In geometry this process is described as parallel transport. As an eigenstate $|n(\boldsymbol{R})\rangle$ is parallel transported along a path $C$ it can pick up a phase factor made up of two components, the usual dynamical phase factor which is independent of $C$ and another, the result of the parallel transport, the geometric phase factor. The state at time $t$ can be written

$$
|n(\boldsymbol{R}(t))\rangle=e^{i \gamma_{n}(t)} \exp \left[-i / \hbar \int_{0}^{t} d t^{\prime} \epsilon_{n}\left(\boldsymbol{R}\left(t^{\prime}\right)\right)\right]|n(\boldsymbol{R}(t=0))\rangle
$$

where the term in the square brackets is the dynamical phase factor and is dependent on time $t$. This can be inserted into the Schrödinger equation and operated on by $\langle n(\boldsymbol{R})|$ to find an expression for the geometrical phase factor $\gamma_{n}$ as

$$
\gamma_{n}=i \int_{c} d \boldsymbol{R} A_{n}(\boldsymbol{R}), \quad A_{n}(\boldsymbol{R})=\left\langle n(\boldsymbol{R})\left|\frac{\partial}{\partial \boldsymbol{R}}\right| n(\boldsymbol{R})\right\rangle .
$$

If the Hamiltonian is returned to its original state after some time $t$ (i.e. the path $C$ is a closed loop), and the result of the above equation is some nonzero phase factor, the transport is then said to be nonholonomic. Note that $\gamma_{n}$ depends on the path taken, rather than the time taken as in the dynamical phase factor. This can be most easily seen by considering a vector being transported along the surface of a sphere, as is shown in Figure 4.1. The vector is not allowed to rotate around the normal to the surface but will still be rotated by an angle $\Omega$ when it is returned to its original location. This phase is related to the topology of the surface on which the vector is transported and the path taken.

When the problem is considered in three dimensions Equation 4.2 can be rewritten using Stokes' theorem and may be recast with the integration over a surface 


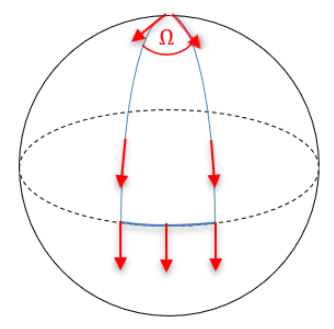

Figure 4.1: Schematic showing the parallel transport of a vector along the surface of the sphere.

$$
\gamma_{n}=\int_{S} d \boldsymbol{A} \boldsymbol{\Omega}_{n} \quad \boldsymbol{\Omega}_{n}=\boldsymbol{\nabla} \times A_{n}(\boldsymbol{R}) .
$$

where $\Omega_{n}$ is referred to as the Berry curvature. The effect of this is to alter the response of an electron to a given potential, and for the case of an electric field results in a velocity given by

$$
\boldsymbol{v}_{n}(\boldsymbol{k})=\frac{1}{\hbar} \frac{\partial \epsilon_{n}(\boldsymbol{k})}{\partial \boldsymbol{k}}-\frac{e}{\hbar} \boldsymbol{E} \times \boldsymbol{\Omega}_{n}(\boldsymbol{k}) .
$$

where the first term is the regular velocity of an electron in Bloch state $\epsilon_{n}(\boldsymbol{k})$ and the second is referred to as the anomalous velocity.

In the classical description it is clear that in a material with an equal number of spin up and spin down electrons, that although each electron will feel a transverse displacement caused by the spin-orbit interaction, there will be no net transverse build-up of electrons. A measurable effect will only be realised in a material with an excess of one spin, a magnetic material. The Berry phase description reaches the same conclusion by consideration of the symmetries of Equation 4.4. The velocity formula should be invariant under time or space inversion if the material in which the electrons are moving possesses these symmetries. Under time reversal $v \rightarrow-v, k \rightarrow-k$ but $\mathbf{E}$ is not inverted $\mathbf{E} \rightarrow \mathbf{E}$. Thus time-reversal makes the following requirements of Equation 4.4:

$$
\Omega_{n}(-\boldsymbol{k}) \rightarrow-\boldsymbol{\Omega}_{n}(\boldsymbol{k})
$$


Under inversion symmetry $v \rightarrow-v, k \rightarrow-k$ and $E \rightarrow-E$ which requires

$$
\boldsymbol{\Omega}_{n}(-\boldsymbol{k}) \rightarrow \boldsymbol{\Omega}_{n}(\boldsymbol{k})
$$

Here we see that in materials with time and inversion symmetry the Berry curvature must be zero. This condition is lifted if time or inversion symmetry is broken, which allows a non-zero Berry curvature and gives rise to the anomalous contribution to Equation 4.4 and the anomalous Hall effect, in ferromagnetic materials.

We now turn to a simple, semi-classical, description of the intrinsic anomalous Hall effect, simliar to that given in Ref. [12]. The Lorentz transformation of the electric field from a stationary ion, viewed from the rest frame of an itinerant conduction electron, results in a circulating magnetic field

$$
\mathbf{B}=-\frac{\mathbf{v}}{c} \times \mathbf{E}
$$

where $\mathbf{v}$ is the velocity of the ion in the rest frame of the electron and $\mathbf{E}$ is the electric field of the ion. The electron with intrinsic magnetic moment $\boldsymbol{\mu}$ will feel a force

$$
\mathbf{F}_{\text {so }}=\nabla(\mu \cdot \mathbf{B})
$$

which has the effect of displacing a charge carrier dependent on the orientation of its spin magnetic moment, even in the absence of an external field. A schematic of the spin-orbit interaction is shown in Figure 4.2 which shows, from the rest frame of the electrons, the lattice ions moving into the page with the resulting circulating magnetic field. The gradient of the dot product of the intrinsic magnetic moment of the electrons and circulating magnetic field of the lattice ions then gives the spin-orbit force.

If an electron is represented by a Bloch wave packet of wave vector $k$ travelling with velocity $\boldsymbol{v}$ we can write this force as the integral of equa- 


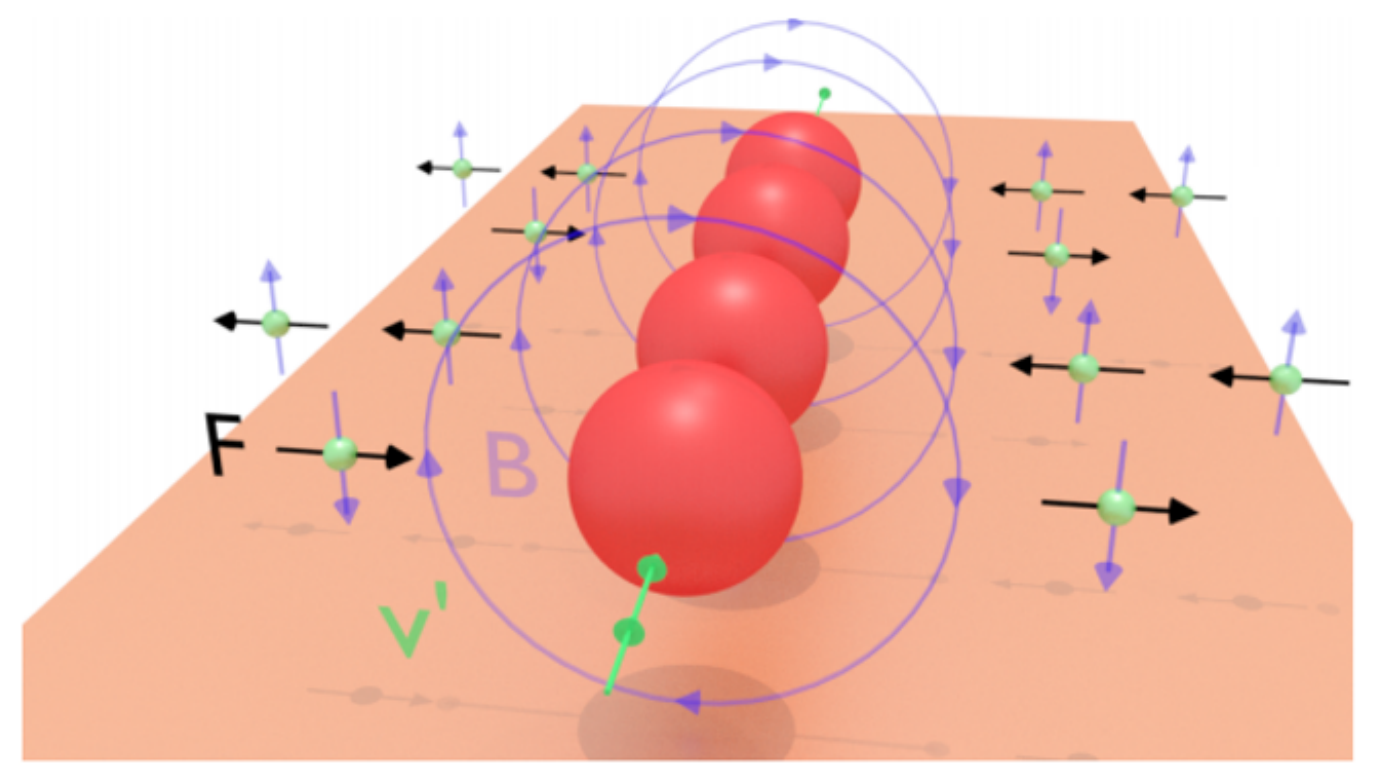

Figure 4.2: Schematic of the spin orbit interaction from the rest frame of the conduction electrons (green). The lattice ions (red) have a relative velocity $\boldsymbol{v}^{\prime}$ into the page causing the circulating magnetic field $\mathbf{B}$. The interaction of this field with the intrinsic magnetic moment of the conduction electrons gives the spin-orbit interaction.

tion 4.6 over the unit cell $u$. Using equation 4.5 , weighted with the conduction electron's wave function $u_{k}$,

$$
\mathbf{F}_{\text {so }}=-\frac{1}{c} \int_{u} d^{3} r \nabla[\boldsymbol{\mu} \cdot(\boldsymbol{v} \times \mathbf{E})]\left|u_{\boldsymbol{k}}\right|^{2}
$$

If we consider a material in the ferromagnetic phase with $\boldsymbol{\mu}=\mu_{z} \hat{\mathbf{z}}$ we select only the $\hat{\mathbf{z}}$ component of the cross product. We then allow the electron velocity in the $\hat{\mathbf{x}}$ or $\hat{\mathbf{y}}$ directions. By considering that $\partial_{x} E_{y}$ and $\partial_{y} E_{x}$ are odd around the centre of inversion of the crystal while $\left|u_{\boldsymbol{k}}\right|^{2}, \partial_{x} E_{x}$ and $\partial_{y} E_{y}$ are even, the former do not contribute to the integral. The force from the spin-orbit interaction can then be written as: 


$$
\begin{aligned}
& \mathrm{F}_{\mathrm{x}}=-\frac{\mu_{z} v_{y}}{c} \int_{u} d^{3} r \partial_{x} E_{x}\left|u_{\boldsymbol{k}}\right|^{2}, \\
& \mathrm{~F}_{\mathrm{y}}=-\frac{\mu_{z} v_{x}}{c} \int_{u} d^{3} r \partial_{y} E_{y}\left|u_{\boldsymbol{k}}\right|^{2} .
\end{aligned}
$$

Under the cubic symmetry of the rare earth nitrides the three components $\partial_{i} E_{i}$ with $i=x, y, z$ are equal so the above can be rewritten using the gradient of the total electric field with the addition of a factor of $1 / 3$

$$
\begin{aligned}
& \mathrm{F}_{\mathrm{x}}=-v_{y} \frac{\mu_{z}}{3 c} \int_{u} d^{3} r \boldsymbol{\nabla} \cdot \mathbf{E}\left|u_{\boldsymbol{k}}\right|^{2}, \\
& \mathrm{~F}_{\mathrm{y}}=-v_{x} \frac{\mu_{z}}{3 c} \int_{u} d^{3} r \boldsymbol{\nabla} \cdot \mathbf{E}\left|u_{\boldsymbol{k}}\right|^{2},
\end{aligned}
$$

and now using Gauss' law the above can be rewritten using the charge density $\rho(\boldsymbol{r})$ which includes all nuclear and core electron charges:

$$
\begin{aligned}
& \mathrm{F}_{\mathrm{x}}=-\frac{\mu_{z} v_{y}}{3 c} \int_{u} d^{3} r \frac{\rho(\boldsymbol{r})}{\epsilon_{0}}\left|u_{\boldsymbol{k}}\right|^{2}=A_{S O} v_{y} \quad(a) \\
& \mathrm{F}_{\mathrm{y}}=-\frac{\mu_{z} v_{x}}{3 c} \int_{u} d^{3} r \frac{\rho(\boldsymbol{r})}{\epsilon_{0}}\left|u_{\boldsymbol{k}}\right|^{2}=A_{S O} v_{x} \quad(b)
\end{aligned}
$$

We can then consider an electron with mass $m_{e}$ travelling through a material with velocity $\mathbf{v}$ under combined influence of an electric field $\mathbf{E}=$ $\left[\mathrm{E}_{\mathrm{x}}, \mathrm{E}_{\mathrm{y}}, 0\right]$, a magnetic field $\mathbf{B}=\left[0,0, \mathrm{~B}_{\mathrm{z}}\right]$ and the spin orbit interaction $\mathrm{F}_{\mathrm{i}}=$ $\left[A_{S O} v_{y}, A_{S O} v_{x}, 0\right]$. Using equations $4.7(a)$ and $(b)$ we can write the steady state, component wise, equations of motion as

$$
\begin{gathered}
\frac{m_{e}}{\tau} v_{x}=-e \mathrm{E}_{\mathrm{x}}-v_{y}\left(e \mathrm{~B}_{\mathrm{z}}-A_{S O}\right) \\
\frac{m_{e}}{\tau} v_{y}=-e \mathrm{E}_{\mathrm{y}}+v_{x}\left(e \mathrm{~B}_{\mathrm{z}}-A_{S O}\right) \\
\frac{m_{e}}{\tau} v_{z}=-e \mathrm{E}_{\mathrm{z}} .
\end{gathered}
$$

This can be simplified by multiplying by $-e n \tau m_{e}^{-1}$ and recognising that $e B_{z} m^{-1}=\omega_{c}$ the cyclotron frequency, $-n e \boldsymbol{v}=\boldsymbol{j}$ the current density and defining $A_{S O} m_{e}^{-1}=\omega_{S O}$ analogous to the cyclotron frequency. These equations can then be solved for the current density to find the conductivity 
tensor, then inverted to find the measurable resistivity tensor

$$
\boldsymbol{\rho}=\rho_{0}\left(\begin{array}{ccc}
1 & \tau\left(\omega_{c}-\omega_{S O}\right) & \\
\tau\left(\omega_{S O}-\omega_{c}\right) & 1 & 0 \\
0 & 0 & 1
\end{array}\right)
$$

Here we see that the spin-orbit interaction simply adds an extra term to the off-diagonal matrix elements, dependent on $\omega_{S O}$.

\subsection{Motivation}

The anomalous Hall effect has generally been studied in metallic systems $[76,78,79,80,81,82]$ where, inspired by theory, the three contributions are thought to scale variously with the longitudinal resistivity of the material $\rho_{x x}$. The longitudinal resistivity is used as a proxy for the scattering time $\tau$ due to the fixed carrier concentrations in metallic systems. The ferromagnetic semiconductors of the rare earth nitride series offer more control and allow the investigation of the anomalous Hall effect with an independent control of scattering time $\tau$ and carrier concentration $n$. Experiment has so far uncovered a clear relationship between the magnitude of the anomalous Hall effect and carrier concentration in GdN [12], rather than the commonly quoted $\rho_{x x}$ proportionality.

Increasing the scope of this investigation to other rare earth nitrides can provide information regarding the conduction channel in these materials. This can be seen most easily by comparison with the GdN case. The simple $4 f^{7}$ electronic configuration of the half-filled $4 f$ shell gives GdN a strong magnetisation and small coercive field. The lack of an orbital contribution to the magnetisation furthermore simplifies the interpretation of the anomalous Hall effect. GdN is in some ways similar to the systems in which the anomalous Hall effect has historically been studied, the ferromagnetic transitions metals, where the orbital angular momentum is nonzero yet quenched. This orbital quenching is reduced in the rare earth 
nitrides due to the smaller radius of the $4 f$ wave function. Furthermore, the interplay of spin and orbital magnetism has interesting consequences for the anomalous Hall effect which is apparent in the lighter members of the rare earth nitride series.

For systems with electron shells less than half filled, in a simple Hund's rules picture (discussed in Section 2.1.2) the spin and orbital contributions to the magnetisation are anti-parallel. Considering the rare earth nitrides, PrN and NdN now stand out as having a magnetisation which is dominated by the orbital component. In an external field it is then the net magnetisation which is aligned by the Zeeman interaction leaving the spin contribution aligned anti-parallel to the external field. This now has consequences for the conduction electrons. For the case of conduction in a $5 d$ band, the strong $4 f / 5 d$ exchange interaction aligns the conduction electrons' spin with the spin component of the $4 f$ electron shell. For the case of conduction in a $4 f$ band, the exchange interaction will again align the itinerant electrons' magnetic moments with those of the localised $4 f$ electrons in the valence band. These materials now find themselves in the peculiar situation of having the conduction electron spins aligned in the opposite direction to an applied field and the sample's net magnetisation. SmN is another interesting case where Hund's rules predict a zero net magnetisation. Experiment has found a small residual moment which is again orbital dominated [52]. NdN and $\mathrm{SmN}$ now provide a strong contrast to GdN where the half filled $4 f$ shell provides a strong spin dominated magnetisation, with no orbital contribution.

Equations $4.7(a)$ and $(b)$ show that the direction of the force from the spin orbit interaction is determined by the orientation of the conduction electron's magnetic moment. The magnitude of the force is proportional to the product of the charge density $\rho(\boldsymbol{r})$ and the conduction electron's wave function $\left|u_{k}\right|^{2}$, which leads to a similar proportionality for the transverse resistivity 


$$
\rho_{x y} \propto \int d^{3} r \rho(\boldsymbol{r})\left|u_{k}\right|^{2}
$$

Both the $5 d$ and the $4 f$ wave functions, the candidates for conduction in the rare earth nitrides, have no weight at all on the lanthanide nucleus. The above integral is then necessarily negative in the rare earth nitride materials. This leads to a negative sign of the anomalous Hall effect for electrons with their spin magnetic moment aligned with the applied magnetic field, and a positive sign for materials with conduction electron spins anti-aligned [12].

The magnitude of the anomalous Hall effect is largely determined by the weight of $\left|u_{k}\right|^{2}$ close to the nucleus, as this is where the majority of the core-electron charge density resides. The $4 f$ wave function is weighted at a substantially smaller radius than the $5 d$, so that the magnitude of the anomalous Hall effect serves to identify which band forms the transport channel.

\subsection{Experimental Techniques}

Measurements of the anomalous Hall effect were conducted in a similar fashion to measurements of the ordinary Hall effect described in Section 3.3.1 where the separation of the transverse Hall signal $\mathrm{R}_{x y}$ from the parasitic longitudinal signal $\mathrm{R}_{x x}$ is first described. The subtraction procedure previously described of measuring the sample in positive and negative field is now less trivial as it is the magnetisation, not only the applied field which now must be reversed. The problem is again exaggerated in $\mathrm{SmN}$, for which the small magnetisation brings with it a large coercive field and significant magnetic hysteresis.

To account for the parasitic longitudinal contribution $\mathrm{R}_{x x}$ to the measured Hall resistance $\mathrm{R}_{x y}$ one must note that below the Curie temperature the contribution from the anomalous Hall effect is odd in magnetisation $\mathbf{M}$ 
rather than the applied field $\mathbf{B}$, while the magneto-resistance $\mathbf{R}_{x x}$ is even in both $\mathbf{M}$ and $\mathbf{B}$.

$$
\begin{gathered}
\mathrm{R}_{x y}(\mathbf{B}, \mathbf{M})=-\mathrm{R}_{x y}(-\mathbf{B},-\mathbf{M}) \\
\mathrm{R}_{x x}(\mathbf{B}, \mathbf{M})=\mathrm{R}_{x x}(-\mathbf{B},-\mathbf{M})
\end{gathered}
$$

To perform a successful separation of $\mathrm{R}_{x x}$ and $\mathrm{R}_{x y}$ one must reverse both $\mathbf{M}$ and $\mathbf{B}$, which can be achieved by completing a full hysteresis loop. This is possible for many of the rare earth nitride materials easily down to temperatures of $\sim 2 \mathrm{~K}$ (i.e. GdN, where the coercive field is on the order of 100 Oe). In materials with a small coercive field a simple field sweep is enough to collect the data required for a subtraction. The remaining discussion focuses on the specific techniques required for measuring the anomalous Hall effect in SmN, where the coercive field below $15 \mathrm{~K}$ is large enough that a field sweep of $\pm 9 \mathrm{~T}$ (the maximum available in our system) cannot saturate the magnetisation.

To begin it should be noted that full hysteresis loops are possible in $\mathrm{SmN}$ between $20 \mathrm{~K}$ and $30 \mathrm{~K}$. For these temperatures samples were prepared in a saturated state by cooling in a field of $9 \mathrm{~T}$. The field was then cycled from $+9 \mathrm{~T}$ to $-9 \mathrm{~T}$, and back to $+9 \mathrm{~T}$. The left-hand panel of Figure 4.3 shows such a measurement where the full hysteresis loop is completed. Fig. 4.3L(a) shows signs of an even longitudinal contribution which can be seen represented by two peaks separated by magnetic hysteresis near $\pm 1 \mathrm{~T}$. The odd component, the anomalous Hall effect, reduces the height of one of these peaks. Taking the difference between points at $(\mathbf{B}, \mathbf{M})$ and $(-\mathbf{B},-\mathbf{M})$ yields Fig. 4.3L(b), the anomalous Hall effect, while the sum yields Fig. 4.3L(c), the magneto-resistive contribution.

At temperatures below $15 \mathrm{~K}$, where the field required to fully reverse the magnetisation is larger than the $9 \mathrm{~T}$ available, a full reversal of the sample magnetisation is not possible without cycling the sample temperature to near the Curie temperature and cooling again in an appropriate field. In 

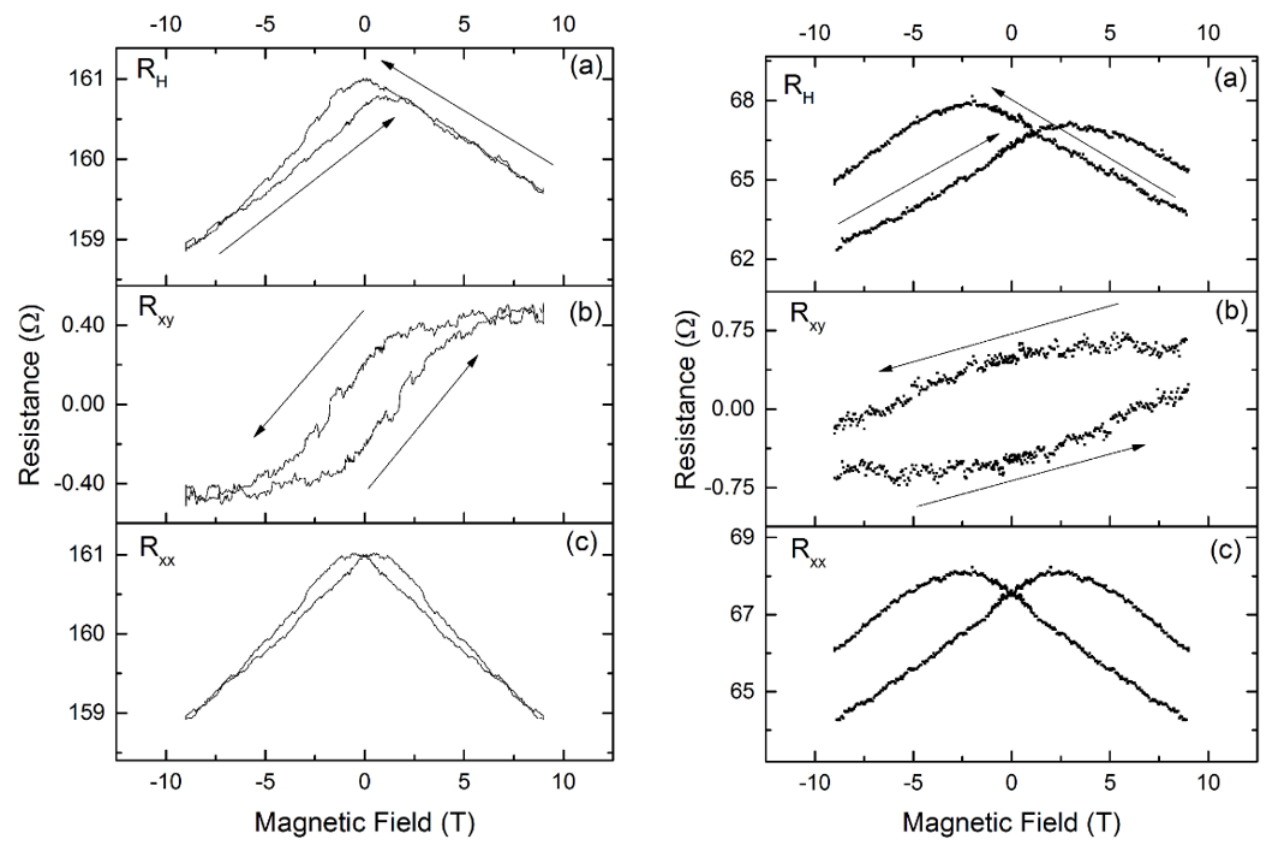

Figure 4.3: Left: Hall measurement taken at $20 \mathrm{~K}$ in a $\mathrm{SmN}$ film. $\mathrm{R}_{H}$ typically contains some contribution from the longitudinal resistance of the sample, $\mathrm{R}_{x x}$, as seen in panel $\mathrm{L}(\mathrm{a})$. This may be separated into odd contributions $\mathrm{R}_{x y}$ [panel $\mathrm{L}(\mathrm{b})$ ] and even contributions $\propto \mathrm{R}_{x x}$ [panel L(c)]. In this sample a full hysteresis loop is completed at the measurement temperature. Right: Measurement on a sample at $10 \mathrm{~K}$, the subtraction procedure at these low temperatures is described in the text.

this temperature range samples were cooled from a temperature of $\sim 30 \mathrm{~K}$ in a field of $+9 \mathrm{~T}$ to ensure full magnetisation, the field was then swept to $-9 \mathrm{~T}$ while Hall measurements were made. Samples were then heated to $30 \mathrm{~K}$ and cooled under a field of $-9 \mathrm{~T}$, saturating the magnetisation in the opposite direction to the previous measurement. The measurements were then repeated while sweeping the field back to +9 T. Although a full hysteresis loop was not completed, for any measurement at $(\mathbf{B}, \mathbf{M})$ at a given temperature there exists a corresponding measurement at $(-\mathbf{B},-\mathbf{M})$, enabling the separation of $\mathrm{R}_{x x}$ from $\mathrm{R}_{x y}$ as in the higher temperature mea- 


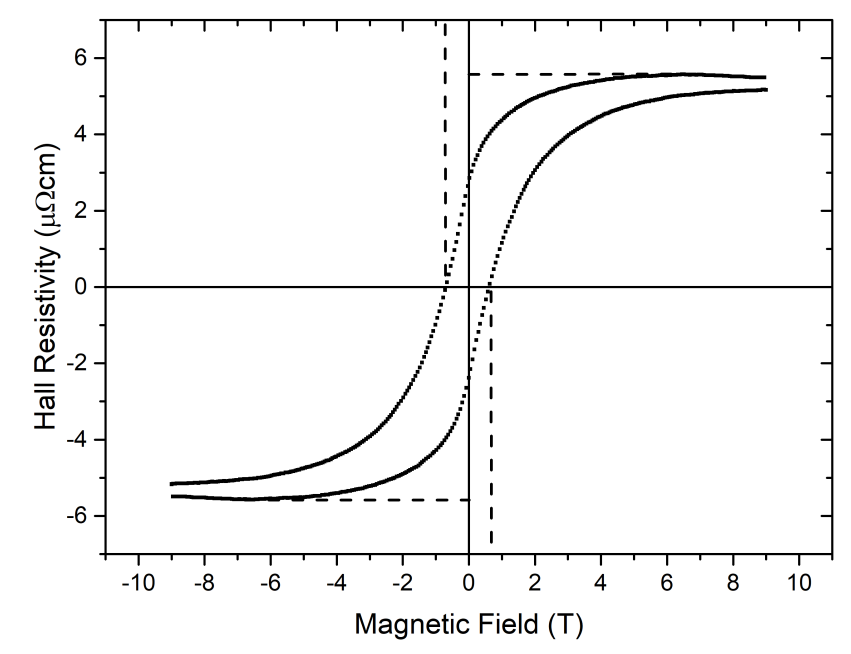

Figure 4.4: Hall measurements on a SmN sample (R015) at $15 \mathrm{~K}$ after the subtraction process has been completed. The transverse resistivity maximises near $\pm 6 \mu \Omega \mathrm{cm}$ which is taken as the magnitude of the anomalous Hall effect, this is indicated by horizontal dashed lines. The coercive field is estimated by the dashed vertical lines.

surements. The right-hand panel of Figure 4.3 shows an example of this process at $10 \mathrm{~K}$. The discontinuities at $\pm 9 \mathrm{~T}$ in Fig. 4.3R(a) give an indication of the field strength that would be required to complete the hysteresis loop. By completing a subtraction as previously described the anomalous Hall effect can be obtained as in Fig. 4.3R(b), with the sum yielding the magneto-resistive contribution in Fig. 4.3R(c).

The magnitude of the anomalous Hall effect can be determined by extrapolation of the saturated signal at high field. This is completed in both positive and negative field and an average taken as a measurement of the magnitude at a given temperature. An estimate of the coercive field can also be determined via measurement of the anomalous Hall effect. The coercive field is defined as the external field required to drive the previously saturated magnetisation to zero. This is not strictly the same as the field required to drive the transverse resistance $\mathrm{R}_{x y}$ to zero, as the anomalous Hall effect is driven by the spin imbalance rather than the total magnetisation, 
furthermore, at any finite field there is a contribution from the ordinary Hall effect. In any case the field at which $\mathrm{R}_{x y}=0$ is a good indication of the coercive field. Again this procedure is completed for both positive and negatives fields and an average taken as the coercive field at a given temperature. An example of this process is shown in Figure 4.4 for a measurement of a $\mathrm{SmN}$ sample at $15 \mathrm{~K}$.

\subsection{Results and Discussion}

\subsubsection{Samarium Nitride}

Measurements of the anomalous Hall effect were conducted in a series of SmN samples. The left-hand panel of Figure 4.5 shows measurements conducted on a single sample, which provides a representative example of the series. The positive sign of the anomalous Hall effect is consistent with the semi-classical description given in section 4.2 in the context of the orbital dominated magnetism of $\mathrm{SmN}$. This can be seen most easily in the right-hand panel of Figure 4.5 where a Hall measurement is shown at $200 \mathrm{~K}$, well above the Curie temperature of SmN, along with a Hall measurement at $30 \mathrm{~K}$ which is near the Curie temperature. The right-hand panel of Figure 4.5 shows a negative slope for the ordinary Hall effect in the data at $200 \mathrm{~K}$, while the $30 \mathrm{~K}$ data show a positive slope for the anomalous Hall effect at low applied field. In the $30 \mathrm{~K}$ data the anomalous Hall effect is seen to saturate at $\sim 4 \mathrm{~T}$ after which the ordinary Hall effect is apparent. Furthermore there is a large difference in the gradient of the ordinary Hall effect between the measurements at these two temperatures. The lower temperature measurement shows a reduced slope by a factor of $\sim 3$. If these slopes are considered in the simple parabolic Hall effect model this signals an increase in carrier concentration by a factor of $\sim 3$, relying of course on a saturated magnetic state above $\sim 4 \mathrm{~T}$.

The lowest temperature measurement at $2 \mathrm{~K}$ in the left-hand panel of 

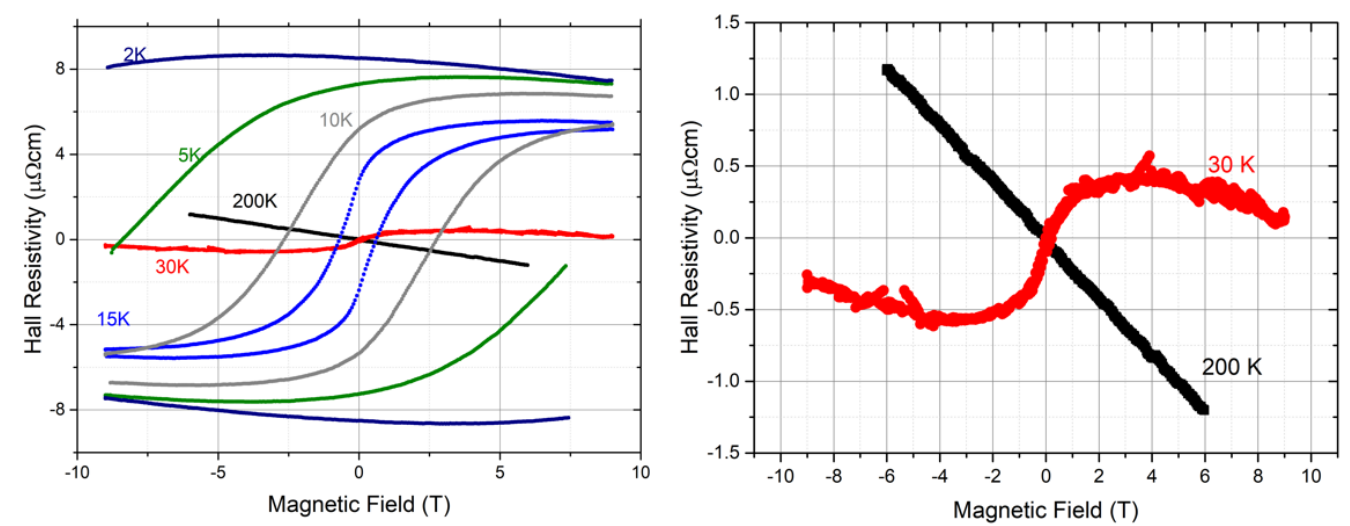

Figure 4.5: Left: Plot of Hall resistivity at a function of applied magnetic field in a SmN sample (R015) for one temperature above the magnetic transition, and several below. The magnitude of both the anomalous Hall effect and the coercive field can be seen to increase as temperature decreases. Right: The $200 \mathrm{~K}$ and $30 \mathrm{~K}$ data from the left panel are shown alone to clearly display the differing sign of the ordinary and anomalous Hall effects. The slope of the $200 \mathrm{~K}$ data and the high field slope of the $30 \mathrm{~K}$ data are inversely proportional to the carrier concentration of the sample at these temperatures and show a three-fold increase in carrier concentration at the lower temperature.

Figure 4.5 shows more complex behaviour. When the field is first swept from $+9 \mathrm{~T}$ there is initially a negative slope. At these low temperatures the coersive field of $\mathrm{SmN}$ is so large the spin imbalance is not affected by the applied field until it has been swept from $+9 \mathrm{~T}$ to $\sim-3 \mathrm{~T}$. Before $-3 \mathrm{~T}$ is reached the external field is not strong enough to begin to alter the magnetic alignment thus the anomalous Hall effect is not changing and all we observe is the negative slope of the ordinary Hall effect, offset by the saturated anomalous contribution. From a linear fit to this negative slope at high fields an estimate of the carrier concentration can be made at the lowest temperatures. The reduced slope, compared to the $200 \mathrm{~K}$ measurement, indicates an increase in carriers at $2 \mathrm{~K}$ by a factor of $\sim 2$, similar to, but somewhat less than, the $30 \mathrm{~K}$ measurement. The positive sign of the anomalous Hall effect, seen clearly in Figure 4.5, is another confirmation 

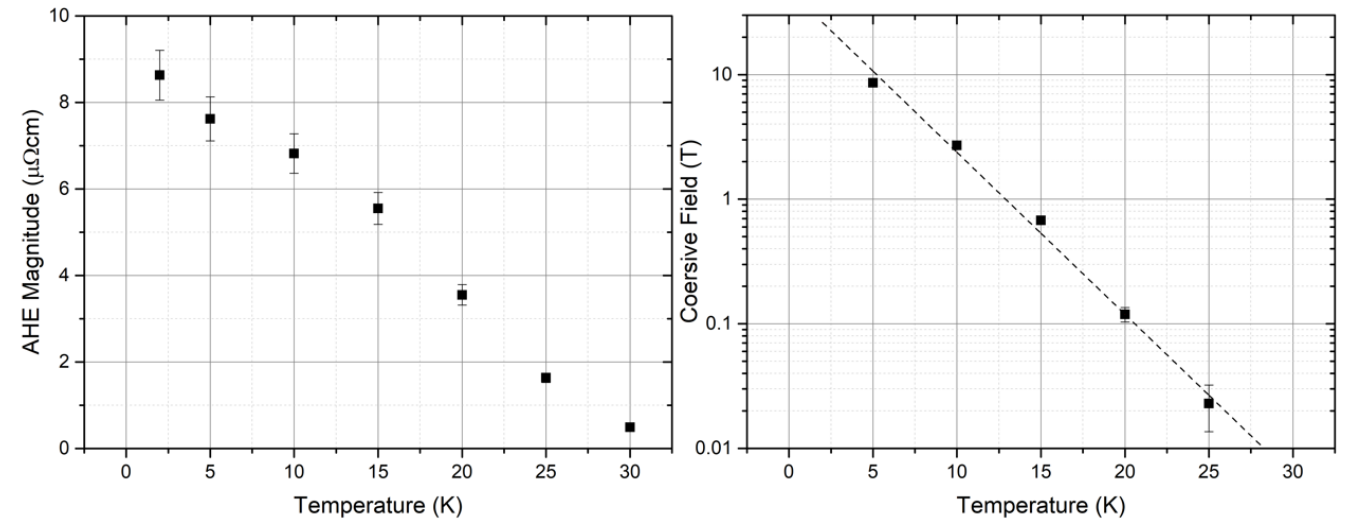

Figure 4.6: Left: The magnitude of the anomalous Hall effect as a function of temperature for the sample shown in Figure 4.5. Right: Plot of the coercive field as a function of temperature for the sample shown in Figure 4.5. The coercive field can be seen to increase approximately exponentially as temperature is decreased.

of the opposing spin and orbital moments in $\mathrm{SmN}$, and consistent with previous XMCD measurements [52].

The left-hand panel of Figure 4.6 shows the magnitude of the measurements shown in Figure 4.5 as a function of temperature. The anomalous Hall effect can be seen to increase from a near zero value at $30 \mathrm{~K}$, where ferromagnetic alignment begins, and to saturate for temperatures below $15 \mathrm{~K}$. The anomalous Hall effect follows the saturation magnetisation of the material as both should increase with the increased spin alignment achieved at low temperatures. This data is similiar to previous magnetic measurements [65] showing the Curie temperature of SmN of $\sim 27 \mathrm{~K}$. The coercive field can also be estimated from these measurements and is displayed as a function of temperature in the right-hand panel of Figure 4.6. The coercive field can be seen to increase roughly exponentially as temperature falls. The dashed line in the figure represents a linear fit on the log-liner scale which roughly matches the data, although there is still a curve present. The near-zero temperature coercive field is found to be $\sim 50 \mathrm{~T}$. 


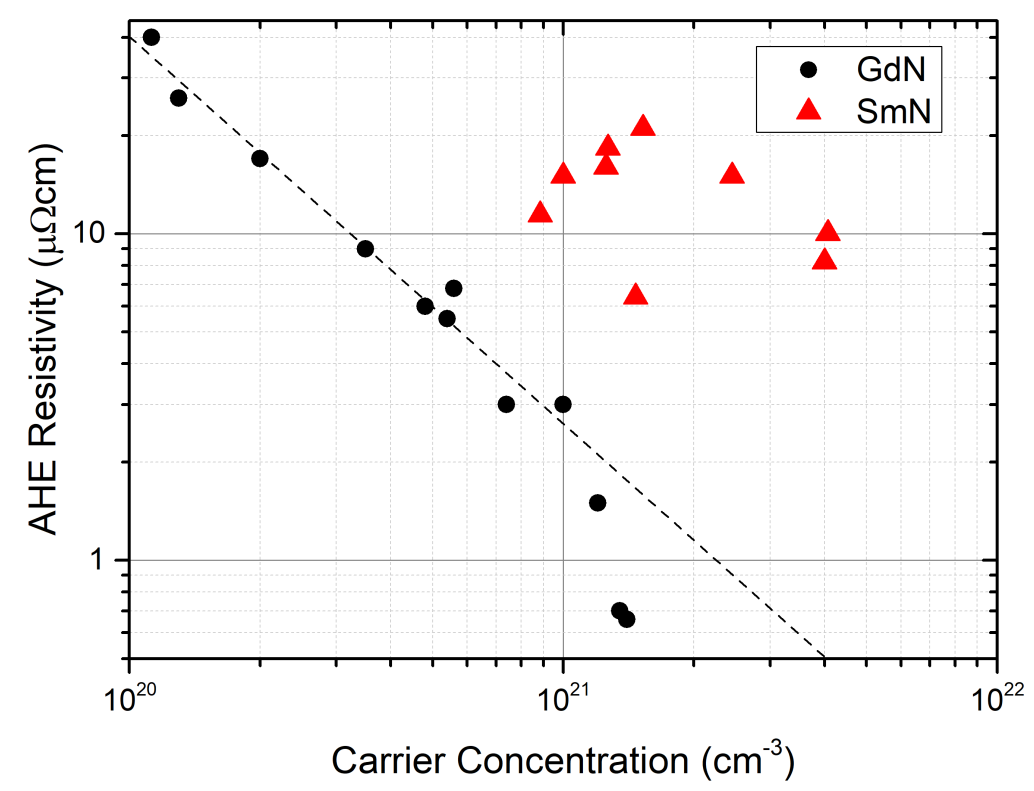

Figure 4.7: The magnitude of the AHE at $2 \mathrm{~K}$ as a function of carrier concentration taken in a series of GdN samples (black circles [taken from Ref. [12]]) and the present $\mathrm{SmN}$ samples (red triangles). The AHE in $\mathrm{SmN}$ is of a significantly larger magnitude than that of $\mathrm{GdN}$ at similar carrier concentration. The $\mathrm{SmN}$ data also show little variation with carrier concentration which contrasts the GdN data with a clear slope of -1 on a log-log scale.

We now move from an analysis of the properties of a single film to the entire series of $\mathrm{SmN}$ films measured. First, we consider the magnitude of the anomalous Hall effect in this series of $\mathrm{SmN}$ films, and compare the present results to measurements in GdN films, taken from Ref. [12]. The magnitude of the anomalous Hall effect in this series of $\mathrm{SmN}$ films, taken at $2 \mathrm{~K}$, is presented in Figure 4.7 as a function of carrier concentration. The carrier concentration was measured well above the Curie temperature to avoid influence from the non-saturated anomalous Hall effect. The SmN data in Fig. 4.7 show a magnitude of $\sim 15 \mu \Omega \mathrm{cm}$ with a spread over a factor of $\sim 3$. Comparing measurements of $\mathrm{SmN}$ (red triangles) to GdN (black circles), taken well below the Curie temperature, an enhancement in size of close to an order of magnitude can be seen between films of comparable 
carrier concentration. The measured anomalous Hall effect in $\mathrm{SmN}$ is then significantly larger than would be expected based on the $5 d$ conduction scenario of GdN [12]. This increased magnitude is indeed expected to occur for conduction within a $4 f$ band. We now address this in the context of the band structure of $\mathrm{SmN}$.

The band structure of $\mathrm{SmN}$ features two empty majority-spin $4 f$ bands, which calculations find near the conduction band minimum [2]. Interpretation of the observed superconductivity in SmN [58] is in terms of a $4 f$ band, which would then suggest that the $4 f$ hybridises with, or even lies lower than, the $5 d$ band. The minority-spin $4 f$ band is predicted to lie some $5 \mathrm{eV}$ higher [2], ensuring that any $4 f$ conduction is by majority-spin electrons alone.

In Section 4.2 it was shown that the magnitude of the anomalous Hall effect is proportional to an integral over the unit cell of the product of the conduction electron's wave function and the atomic charge density. It is possible, using a simple calculation, to compare the expected relative magnitudes of the anomalous Hall effect for the cases of $5 d$ and $4 f$ conduction in $\mathrm{SmN}$ to the $5 d$ conduction channel in GdN.

Equation 4.8 was calculated for the cases for $4 f$ and $5 d$ conduction in $\mathrm{SmN}$ and $5 d$ conduction in $\mathrm{GdN}$ using a simple hydrogenic atomic model where each wave function $u_{k}$ is a radial solution of the hydrogen atom. When calculating the charge density of the inner electronic states, atomic screening constants from Clementi et al. $[86,87]$ were used. A full $\mathrm{L}^{3+}$ configuration was used in each case, which excluded the two $6 s$ electrons and one $4 f$ electron for the Sm and two $6 s$ electrons and one $5 d$ electron for $\mathrm{Gd}$. The screening constant for the Sm $4 f$ electrons was available. However, $\mathrm{Sm} / \mathrm{Gd} 5 d$ screening constants were not. To approximate the screening constants for the $5 d$ orbitals the lowest available $5 d$ screening constant ( $\mathrm{Lu}$ ) was used.

Panel (a) of Figure 4.8 shows the calculation of the electron cloud probability density and probability density of each wave function (normalised 

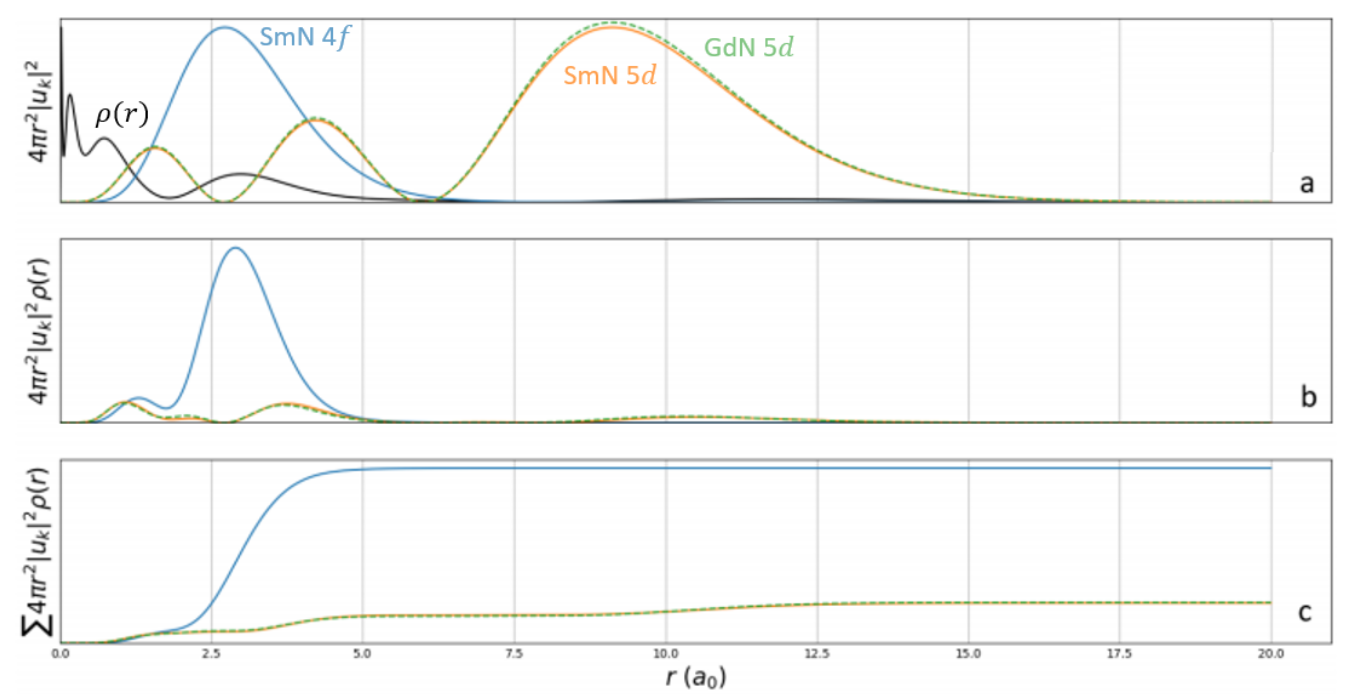

Figure 4.8: Panel (a): Calculation of the probability density of the electron cloud (black), Sm $4 f$ conduction electron (blue), Sm $5 d$ (orange) and Gd $5 d$ (green dashed) probability density (normalised to the maximum of electron cloud probability density). Panel (b): Product of each wave function's probability density with the electron cloud probability density. Panel (c): Running sum of each series in panel (b), the final value for each being the result of the integral of Equation 4.8.

to the maximum value of the electron cloud probability density) for the cases of Sm / Gd $5 d$ and Sm $4 f$ conduction. The calculated electron cloud probability densities (black) for the different configurations are indistinguishable at the scale of the figure. The Sm $4 f$ probability density (blue) has significantly more weight closer to the nucleus, nearer the largest contribution to the core electron probability density. The Sm / Gd $5 d$ probability densities (orange / green-dashed), have a small peak closer to the centre than the Sm $4 f$ but contain the majority of their weight at greater distance.

Panel (b) shows the product of the electron cloud probability density and the conduction electron probability density for each case. The effect of the increased weight of the $4 f$ wave function closer to the nucleus can be seen here in the large peak present in the Sm $4 f$ series. Panel (c) shows the 
running sum of panel (b), the final value being the result of Equation 4.8 for the three cases considered.

The ratio of the integral for $\mathrm{Sm} / \mathrm{Gd} 5 d$ is $\sim 1$, demonstrating the anomalous Hall effect is expected to have a similar magnitude if both materials were to have a $5 d$ conduction channel. The ratio of Sm $4 f / \mathrm{Sm} 5 d$ is $\sim 4$ showing a larger anomalous Hall effect is expected for $4 f$ conduction in SmN. When the data in Figure 4.7 are considered in this context it seems reasonable that the increased magnitude of the $\mathrm{SmN}$ data does indeed signal conduction in a $4 f$ channel.

Figure 4.7 shows a clear proportionality between the magnitude of the anomalous Hall effect and the carrier concentration in GdN. The reason for this, described in detail in Ref. [12], can be seen by considering the spin-orbit interaction which will have opposite contributions for electrons of different spins. We can see this proportionality appear in the transverse resistivity by inverting the conductivity tensor and writing the conductivity as proportional to the carrier density (in terms of each spin channel) to show

$$
\rho_{x y}=\frac{\sigma_{x y}}{\sigma_{x x}^{2}-\sigma_{x y}^{2}} \propto \frac{\left(n_{\uparrow}-n_{\downarrow}\right)}{\left(n_{\uparrow}+n_{\downarrow}\right)^{2}},
$$

where $n_{\uparrow}$ and $n_{\downarrow}$ are the carrier concentrations of spin up and spin down electrons respectively, the proportionality in the equation holds for $\sigma_{x y} \ll$ $\sigma_{x x}$. If, as is the case for GdN at moderate doping levels, the bottom of the conduction band is expected to be occupied by only majority spin charge carriers, i.e. $n_{\downarrow}=0$, then $\rho_{x y} \propto 1 / n$. This proportionality has been shown to hold in GdN [12] for doping levels up to $\sim 10^{21} \mathrm{~cm}^{-3}$ at which point the minority spin band begins to fill. The addition of minority spin carriers then causes the sharp decrease in the magnitude of the anomalous Hall effect near $n=10^{21} \mathrm{~cm}^{-3}$ in Figure 4.7.

The lack of a $1 / n$ proportionality in the $\mathrm{SmN}$ data is interesting and points towards a more complex situation than is the case in GdN. If dopant electrons find a $4 f$ band near the conduction band minimum they will 
likely be somewhat localised due to the reduced radius of the $4 f$ wave function. This may result in some degree of $\mathrm{Sm}^{2+}$ and thus a mixed valence in the material. In a simple Hund's rules picture the $\mathrm{Sm}^{2+}$ ion has $J=|L-S|=0$ and is not expected to align magnetically. This may then have some bearing on the lack of proportionality in the data.

It is also interesting that at the measured carrier concentrations of the $\mathrm{SmN}$ films the net magnetisation remains orbital dominated (i.e. the sign of the anomalous Hall effect remains positive). A simple calculation finds that at doping levels of $\sim 1 \%$ the small magnetisation of $\mathrm{SmN}$ is expected to become dominated by the spin component. That is if all these $\sim 10^{21} \mathrm{~cm}^{-3}$ electrons align with spin moments parallel to that of the filled $4 f$ states. Spin dominated magnetism has not been observed in even the most heavily doped films. Although far from conclusive this again may indicate some influence of the $(J=0) \mathrm{Sm}^{2+}$ ion.

\subsubsection{Neodymium Nitride}

Hall effect and magnetisation measurements were carried out on $\mathrm{NdN}$ samples in a manner similar to $\mathrm{SmN}$. The reduced coercive field of $\mathrm{NdN}$, when compared to $\mathrm{SmN}$, simplified measurements, however, the magnetisation reversal technique described in Section 4.3 was still used below $20 \mathrm{~K}$. The result of the subtraction procedure can be seen for a NdN sample in Figure 4.9.

To begin we can consider the measurement at $300 \mathrm{~K}$ which shows a linear trend with a negative slope, as is expected for all the rare earth nitrides, and is indicative of negative charge carriers present in the conduction band. The slope here gives a carrier concentration of $4.9 \pm 1 \times 10^{21} \mathrm{~cm}^{-3}$, this large carrier concentration is consistent with the small resistivity of $0.35 \pm 0.07 \mathrm{~m} \Omega \mathrm{cm}$, which results in a scattering time of $2.1 \pm 0.9 \times 10^{-15} \mathrm{~s}$. The influence of the anomalous Hall effect can be seen below the magnetic transition temperature of $\sim 45 \mathrm{~K}$. The $30 \mathrm{~K}$ data in Figure 4.9 show 


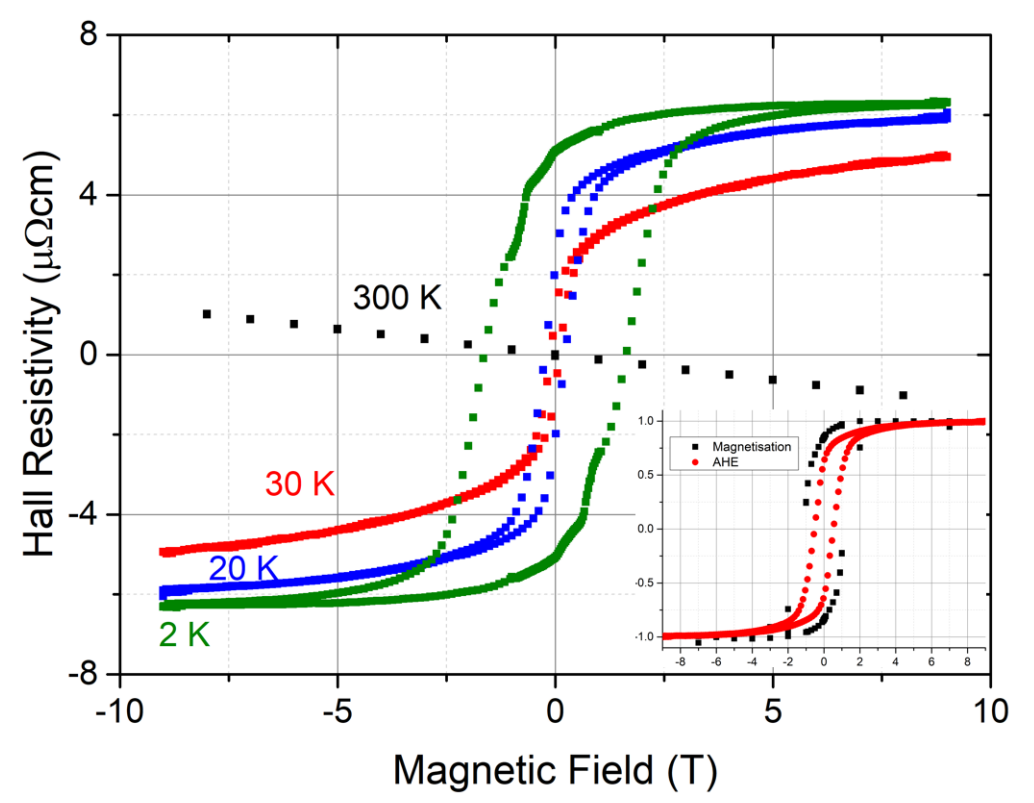

Figure 4.9: Hall effect measurements on a NdN film (A613) show a negative gradient at $300 \mathrm{~K}$ above the Curie temperature. Lower temperature measurements show the anomalous Hall effect with a positive sign and significant hysteresis. Inset: Comparison of the normalised magnetisation and Hall effect measurements as a function of field at $10 \mathrm{~K}$.

the anomalous Hall effect has a clear positive sign. In the frame work previously discussed, this is in line with the expectation of a material where a conduction electron's magnetic moment is anti-parallel to the netmagnetisation, or orbital dominated magnetism in the $4 f$ shell. At lower temperatures the magnitude of the anomalous Hall effect increases as expected. The inset of Figure 4.9 shows the normalised Hall resistivity and a magnetisation measurements of the sample at $10 \mathrm{~K}$, as a function of applied field. These measurements show a similar form, although the coercive fields are different with the magnetic measurement showing a larger coercive field by a factor of $\sim 2$.

We now move to the magnitude of the anomalous Hall effect in $\mathrm{NdN}$ and compare this to $\mathrm{SmN}$ and GdN. The anomalous Hall effect saturates at 

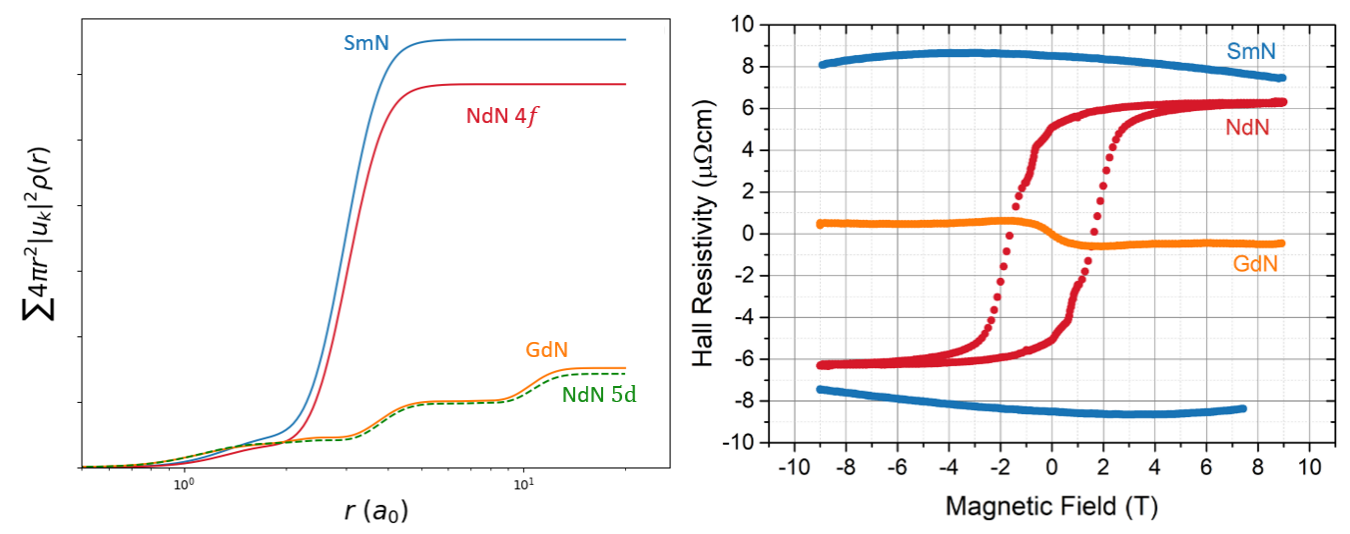

Figure 4.10: Left: Calculation of Equation 4.8 for $4 f / 5 d$ conduction in $\mathrm{NdN}$, $4 f$ condution in $\mathrm{SmN}$ and $5 d$ conduction in $\mathrm{GdN}$ (see section 4.4.1). Right: Hall measurements in $\mathrm{NdN}, \mathrm{GdN}$ and $\mathrm{SmN}$ at $2 \mathrm{~K}$ in samples of comparable carrier density.

$\sim 6 \mu \Omega \mathrm{cm}$ in the $2 \mathrm{~K}$ measurement shown in Figure 4.9. Comparing this to measurements in $\mathrm{SmN}$ and $\mathrm{GdN}$ at comparable carrier concentrations in Figure 4.7 , we see that $\mathrm{NdN}$ reaches $\sim 60 \%$ of the $\mathrm{SmN}$ magnitude while is at least an order of magnitude larger than the most comparable GdN measurement.

We can consider this in the same framework discussed for $\mathrm{SmN}$ and calculate Equation 4.8 for the cases of $\mathrm{Nd} 4 f / 5 d$ conduction. The results of the calculation are shown in the left-hand panel of Figure 4.10, the right-hand panel shows Hall measurements of $\mathrm{SmN}, \mathrm{NdN}$ and $\mathrm{GdN}$ all at $2 \mathrm{~K}$ (in films of comparable carrier concentration). The left-hand panel of Figure 4.10 shows that for the case of $4 f$ conduction the magnitude of the anomalous Hall effect in $\mathrm{NdN}$ is expected to be close to $90 \%$ of the $\mathrm{SmN}$ value. The reason for the calculated decrease in magnitude for the $\mathrm{NdN} 4 f$ case over the Sm $4 f$ case is the increase in the effective charge seen by the $4 f$ wave function in the Sm case, by $\sim 5 \%$. This pulls the $\operatorname{Sm} 4 f$ wave function closer to the atomic nucleus than the $\mathrm{Nd} 4 f$, where the majority of the charge density resides. For the case of $5 d$ conduction in $\mathrm{NdN}$ the 
large radius of the wave function results in a calculated magnitude even smaller than in GdN.

The ratio of the experimental values of the magnitude of the anomalous Hall effect for $\mathrm{NdN}$ and $\mathrm{SmN}$ is $\sim 0.6$, close to the 0.9 calculated. As can be seen in the right-hand panel of Figure 4.10 the experimental value is, in any case, much larger than the measured value for GdN. This provides evidence for at least some influence of the $4 f$ band on the conduction channel in NdN. Calculations [2] have placed the lowest unoccupied $\mathrm{Nd} 4 f$ level variously to meet the $5 d$ at the bottom of the conduction band at the $\mathrm{X}$ point, to some few $\mathrm{eV}$ higher. These results strongly support the case for a low lying $4 f$ band with influence on the transport properties of the material.

\subsubsection{Gadolinium Nitride}

The anomalous Hall effect in GdN has been discussed briefly in Section 4.4.1 and is covered in literature [12]. This section will be limited to the discussion of results collected in a highly conductive GdN film with a measured carrier concentration of $\sim 1.3 \times 10^{21} \mathrm{~cm}^{-3}$.

To begin, measurements of the anomalous Hall effect are shown in Figure 4.11. The left-hand panel of Figure 4.11 shows measurements at various temperatures between $150 \mathrm{~K}$ and $10 \mathrm{~K}$ while the right-hand panel shows the $2 \mathrm{~K}$ measurement alone. All measurements shown in Figure 4.11 have been treated using the subtraction method discussed in section 4.3, the high field slope of the ordinary Hall effect has also been removed leaving the anomalous component, and an additional component at low field in the low temperature data.

We can first note the magnitude of the anomalous Hall effect saturates near $0.5 \mu \Omega \mathrm{cm}$ for the lowest temperatures, which is consistent with the magnitudes of heavily doped GdN films [12]. Moving on we see that the anomalous Hall effect is non-zero at temperatures up to $150 \mathrm{~K}$ showing 

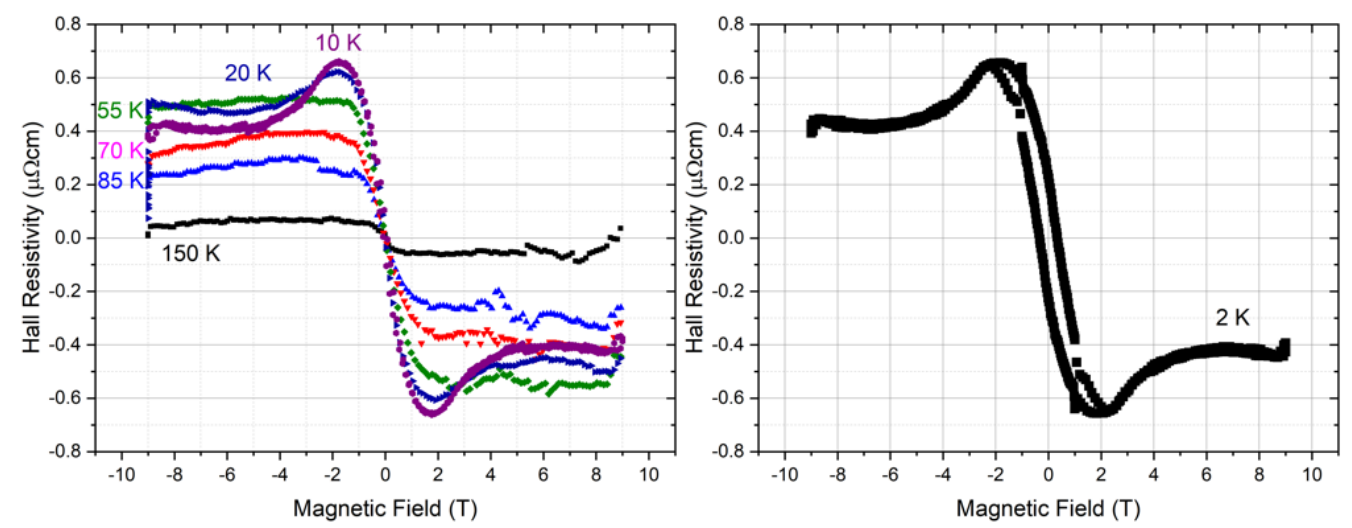

Figure 4.11: Left: Hall effect measurements of a highly conductive GdN sample (A612) which show a non-zero anomalous Hall effect at $150 \mathrm{~K}$. At lower temperatures a lump appears at low magnetic fields reminiscent of a topological Hall effect. Right: A Hall effect measurement at $2 \mathrm{~K}$ on the same film showing significant hysteresis.

that there exists some degree of magnetic order of both the lattice ions and conduction electrons at these high temperatures. Finally, we can note the appearance of a feature near zero field in measurements from $20 \mathrm{~K}$ and below which is reminiscent of a topological contribution to the Hall effect $[88,89,90]$. We first address the appearance of apparent magnetic order at high temperatures with the aid of magnetic measurements.

Plots of the magnetisation and inverse susceptibility as a function of temperature for the present sample are shown in Figure 4.12, measurements shown were taken while the sample was cooled from $250 \mathrm{~K}$ in a field of 200 Oe parallel to the sample plane. The inverse susceptibility in the left-hand panel shows a linear Curie-Wise relationship terminating in a Curie temperature of $\sim 68 \mathrm{~K}$. The magnetisation measurement shows an initial upturn below $\sim 70 \mathrm{~K}$ which appears to roll off before there is an additional contribution near $50 \mathrm{~K}$. These two contributions can be seen more clearly in the temperature derivative of the magnetisation, shown in the right-hand panel of Figure 4.12. Here we see a sharp peak near $70 \mathrm{~K}$ 

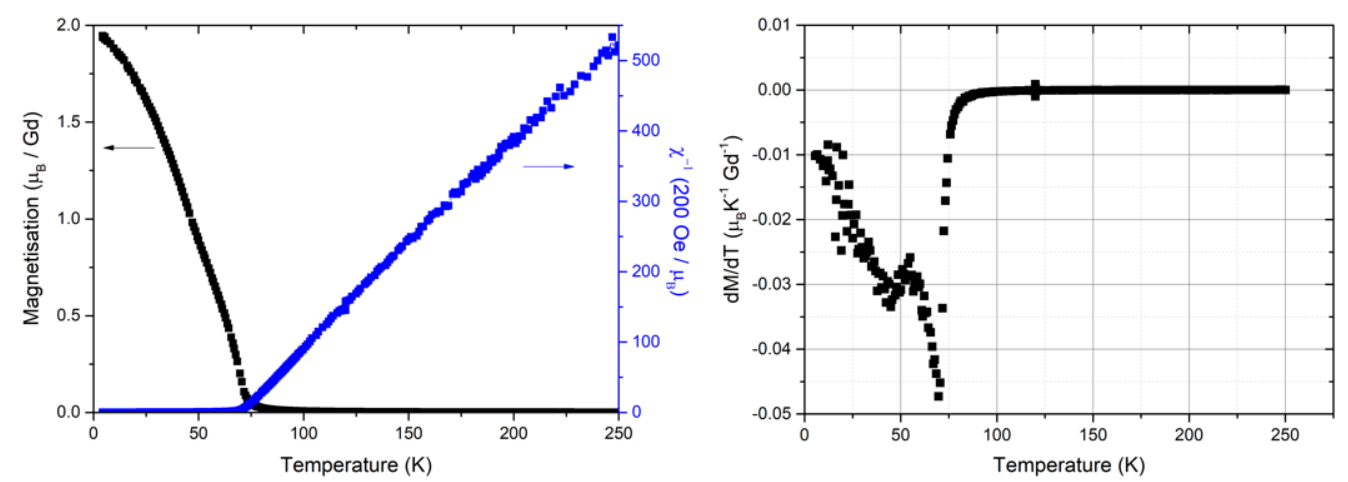

Figure 4.12: Left: Plots of the magnetisation (black) and inverse susceptibility (blue) of a GdN film (A612) as a function of temperature below $200 \mathrm{~K}$. Both measurements indicate a Curie temperature of near $70 \mathrm{~K}$ while the magnetisation measurement shows evidence of a further transition near $50 \mathrm{~K}$. Right: Temperature derivative of the magnetisation data in the left-hand panel, this now more clearly shows the two transitions as a sharp peak near $70 \mathrm{~K}$ and a broader peak near $50 \mathrm{~K}$.

above a second peak near $50 \mathrm{~K}$. The first transition near $70 \mathrm{~K}$ has been linked to the formation of magnetic polarons [49] at nitrogen vacancy sites, which is relevant in the context of this film. An LSDA $+U$ calculation of nitrogen deficient GdN has found two of the three electrons released from the $\mathrm{N}^{3-}$ ion remain localised on the vacancy site while the third is mobile in the conduction band [40]. These weakly bound electrons create an increased electron density near the nitrogen vacancy site which can mediate the ferromagnetic exchange between the $\mathrm{Gd}^{3+}$ ions surrounding the vacancy. The second bump near $50 \mathrm{~K}$ is then the intrinsic ferromagnetic transition of stoichiometric GdN.

We now turn to measurements of the magnetisation as a function of applied field, these are presented in Figure 4.13. The left-hand panel of Figure 4.13 shows the magnetisation as a function of field applied parallel to the sample plane at temperatures from $120 \mathrm{~K}$ to $40 \mathrm{~K}$, the inset shows a measurement with the field perpendicular to the plane at $5 \mathrm{~K}$. To begin we 

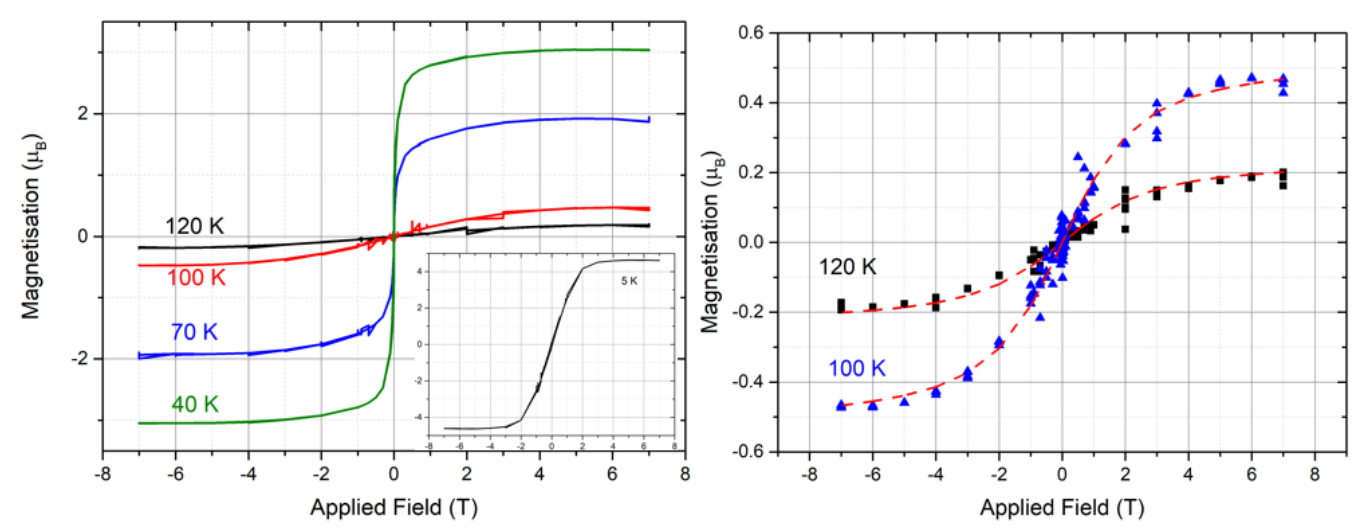

Figure 4.13: Left: Plots of the magnetisation of a function field applied parallel to the samples plane for a GdN sample (A612) from $120 \mathrm{~K}$ to $40 \mathrm{~K}$. The insert shows a measurement at $5 \mathrm{~K}$ with the field perpendicular to the film with clear shape anisotropy. Right: The measurements at $120 \mathrm{~K}$ and $100 \mathrm{~K}$ from the left-hand panel are plotted along with Brillouin functions with $g J \approx 160$, the similarity between the measurement and the model imply the formation of superparamagnetic clusters of $\mathrm{Gd}^{3+}$ ions.

can note that there exists a finite magnetisation even in the highest temperature measurements, similar to the anomalous Hall effect measurements shown in Figure 4.11. This is again significantly above the Curie temperature calculated from the inverse susceptibility in Figure 4.12. The temperature of the measurements and the lack of any hysteresis now points towards a paramagnetic signal, while the magnitude points towards superparamagnetic clusters. This is examined further in the right-hand panel of Figure 4.13 where the measurements at $120 \mathrm{~K}$ and $100 \mathrm{~K}$ are plotted along with Brillouin functions to model the paramagnetic response at each temperature.

The magnetisation $M$ of an ideal paramagnet can be written as a function of applied field $B$ using a Brillouin function $B_{J}(x)$ as 


$$
\begin{gathered}
M=N g J \mu_{B} B_{J}(x), \quad x=\frac{g \mu_{B} J B}{k_{B} T} \\
B_{J}(x)=\frac{2 J+1}{2 J} \operatorname{coth}\left(\frac{2 J+1}{2 J} x\right)-\frac{1}{2 J} \operatorname{coth}\left(\frac{1}{2 J} x\right)
\end{gathered}
$$

with $N$ the number of atoms per volume, $J$ is the total angular momentum and $T$ the temperature. As the number of superparamagnetic clusters is not initially known $B_{J}(x)$ is first normalised to the data then $J$ is determined by finding a model consistent simultaneously with the data at both temperatures. The models in the figure use a value of $g J \approx 160$ where $g$ is the gyromagnetic ratio. These large values for $J$ point to the formation of superparamagnetic clusters, which have a much stronger Zeeman interaction with an external field due to the increased effective moment of the cluster ( $\mu_{\text {cluster }}=n \mu_{\text {ion }}$ where $n$ is the number of ions in a cluster). Assuming these clusters are formed from $\mathrm{Gd}^{3+}$ ions $(J=3.5)$ and $g \sim 2$ this corresponds to clusters of $\sim 23 \mathrm{Gd}^{3+}$ ions or a sphere with a volume of $\sim 6$ unit cells. We can then use the measured magnitude of the saturated signal to roughly estimate the number of clusters or $\mathrm{Gd}^{3+}$ ions contributing to the superparamagnetic signal. Using $g J \sim 160$ this results in $\sim 1 \%$ of $\mathrm{Gd}^{3+}$ ions being involved in a cluster at these temperatures, then assuming one nitrogen vacancy per six $\mathrm{Gd}$ atoms and one electron released into the conduction band per vacancy we find $\sim 1.7 \times 10^{20} \mathrm{~cm}^{-3}$ conduction electrons from cluster related nitrogen vacancies.

Evidence for superparamagnetic regions such as these is furthermore present in Figure 4.12. It is likely the formation of superparamagnetic clusters is linked to that of magnetic polarons whose existence is signalled by the sharp peak in the temperature derivative of the magnetisation, shown in the right-hand panel of Figure 4.12, and discussed in more detail in Ref. [49]. Considering again the anomalous Hall effect it is then not unreasonable to believe that even at high temperatures these clusters of superparamagnetic $\mathrm{Gd}^{3+}$ ions polarise the spin magnetic moment of itinerant conduction electrons leading to an apparent anomalous Hall effect, with 
zero coercive field, at temperatures well above the Curie temperature. The effect seen here is in fact superficially similar to that seen in Hall measurements on some, non-ferromagnetic, dilute Kondo systems which display a non-zero anomalous component to the Hall effect due to polarisation of the local magnetic moments by an applied field $[82,91]$.

The formation of sites of superparamagnetism leads onto a discussion of the additional low field contribution in the Hall measurements at $20 \mathrm{~K}$ and below. This contribution appears similar to a topological Hall effect which arises from an additional Berry phase contribution as conduction electrons pass by sites of smoothly varying magnetic structure [90]. A common example of such a structure leading to a similar effect are skyrmions [92]. These sites of magnetic structure nucleate the formation of magnetic domains. As described above it is reasonable to believe that the sites of superparamagnetism are formed around regions of increased nitrogen vacancy concentration. The increased electron density from these vacancies can mediate exchange between the local $\mathrm{Gd}^{3+}$ ions, this may lead to a stronger exchange interaction in these regions. The stronger exchange can then nucleate the formation of magnetic domains at a low applied field resulting in areas of varying magnetic structure and in turn a topological contribution to the Hall effect.

\subsection{Conclusions}

In conclusion, the positive sign of the anomalous Hall effect in SmN and $\mathrm{NdN}$ indicates that the magnetic moments of the conduction electrons are aligned anti-parallel to the applied field. This is qualitatively expected from Hund's rules and previous experimental results $[52,58]$ showing orbital dominated magnetism in each system.

The enhanced magnitude of the anomalous Hall effect in SmN and $\mathrm{NdN}$, over similar measurements in GdN films, implys the conduction channel in both $\mathrm{SmN}$ and $\mathrm{NdN}$ has influence from a majority spin $4 f$ band. 
A majority spin $4 f$ band in this position is indeed in line with calculations [2] which place unfilled majority spin $4 f$ bands near the conduction band minimum in both materials.

Finally, measurements of the magnetisation and anomalous Hall effect in a heavily doped GdN film indicate the presence of superparamagnetic clusters at temperatures up to $\sim 150 \mathrm{~K}$. These measurements also show a feature that is reminiscent of a topological Hall effect. 


\section{Chapter 5}

\section{Optical Properties of Rare Earth Nitrides}

This chapter describes the measurement and interpretation of the optical properties of various rare earth nitrides. To begin, a motivation for this study is given, which includes a brief review of previous optical studies. The measurement techniques, data processing and modelling are then described. Results begin with undoped $\mathrm{SmN}$ and move on to $\mathrm{NdN}$ samples. This follows on from the previous chapter's discussion relating to the location of $4 f$ levels in the conduction band of $\mathrm{SmN}$ and NdN. The locations of these $4 f$ levels are, in this chapter, determined via optical spectroscopy. Finally, temperature-dependent measurements on GdN and $\mathrm{SmN}$ samples are discussed.

\subsection{Motivation}

The measurement of the optical response of a material is one of the more direct methods to determine its electronic structure. The measurable reflection and transmission spectra lead to the absorption, optical conductivity and dielectric function, which can be directly related to the band structure of the material, as described in Section 2.2.2. 
There have been several experimental and theoretical treatments concerning the optical response of many of the rare earth nitrides. The earliest optical measurements of the rare earth nitrides [93] put the optical gap of GdN, DyN, ErN and HoN near $2 \mathrm{eV}$. This and other previous studies [8,9] of these materials are now thought to have been influenced by oxygen contamination [1], which is difficult to avoid even with current ultra high vacuum technology. The insulating oxides $\mathrm{L}_{2} \mathrm{O}_{3}$ have bandgaps closer to $5 \mathrm{eV}$ [94], which has likely dragged the measured bandgap toward higher energy, as sections of the material became contaminated, in earlier measurements. More recent results point towards a smaller direct optical gap of $\sim 1 \mathrm{eV}$ for most of the members of the rare earth nitride series $[1,2]$.

As is generally the case with most measurement techniques used on the rare earth nitrides, GdN has been the most thoroughly studied. Both experimental $[1,24,60,61,95]$ and theoretical $[1,2,32,37,96]$ work largely agree on a direct bandgap at $\mathrm{X}$ of $\sim 0.9 \mathrm{eV}$ in the ferromagnetic phase and a calculated indirect gap between $\Gamma$ and $X$ of $0.4 \mathrm{eV}$. The strong spin splitting upon cooling into the ferromagnetic phase and reduction of the direct gap has also been seen by several groups [24, 60, 61, 95].

Optical studies of DyN [62] have found a similar bandgap of $1.2 \mathrm{eV}$ and a Moss-Burstein shift of the optical gap when the material is heavily doped with electrons. This study, conducted into the far infra-red regions, also found the location of a phonon absorption near $280 \mathrm{~cm}^{-1}$ close to the predicted location [97]. The choice of YSZ as a substrate in this study did complicate the low energy analysis due to optically active phonons present in the substrate. $\mathrm{NdN}$ [58] has also been investigated experimentally with the identification of a bandgap of $0.9 \mathrm{eV}$ and a strong indication of free carrier absorption in the near infra-red region. This study probed from the ultraviolet to mid infra-red regions as low as $\sim 0.3 \mathrm{eV}$, again the choice of a substrate with optically active phonons complicated lower energy measurements. SmN has seen recent experimental interest [63] with a measured bandgap of $1.2 \mathrm{eV}$ and again a strong indication of free carrier 
absorption, consistent with a Drude picture. Once more, this study was conducted on samples grown on sapphire substrates, with optically active phonons at low energy, prohibiting investigation into the far infra-red region.

It may seem that much of the ground has already been covered with regards to optical measurements. Although investigation of the mid / near infra-red and visible regions has been completed, low energy measurements have not been attempted systematically on appropriate substrates. Investigations of $\mathrm{GdN}, \mathrm{NdN}$ and $\mathrm{SmN}$ at low energy will initially locate phonon absorptions present in these materials, which can be compared with the predicted locations [97]. Measurements at the lowest energies can also be used to identify free carriers, and can be compared to transport measurements. There is also a renewed interest in investigating the band structure, specifically the nature of the band which forms the conduction band minimum in $\mathrm{SmN}$ and $\mathrm{NdN}$, following on from the results presented in Chapter 4 which implied a $4 f$ conduction band minimum in these materials.

The electronic structure of $\mathrm{SmN}$ has been investigated within various density functional theory treatments which show the lowest unoccupied majority-spin $4 f$ band to lie variously from the bottom of the $5 d$ conduction band to some few eV higher [2, 29, 30,37]. This is shown schematically in Figure 5.1 which shows the range of predicted locations for the lowest unoccupied $4 f$ states in $\mathrm{SmN}$ and $\mathrm{NdN}$, along with the $5 d$ band which forms the conduction band minimum across many of the rare earth nitrides. Interestingly none of the predictions place the $\operatorname{Sm} 4 f$ band below the $5 d$, with even the lowest predicted energy [2] leading only to a strong $4 f / 5 d$ hybridisation near the bottom of the $5 d$ band. This hybridisation then repels the main weight of the $5 d$ states to higher energy and leaves the potential of a hybridisation gap above the $4 f$ band. Recent experimental reports of an enhanced anomalous Hall effect [59], and superconductivity [58] in SmN both show some degree of $4 f$ influence on the conduction 


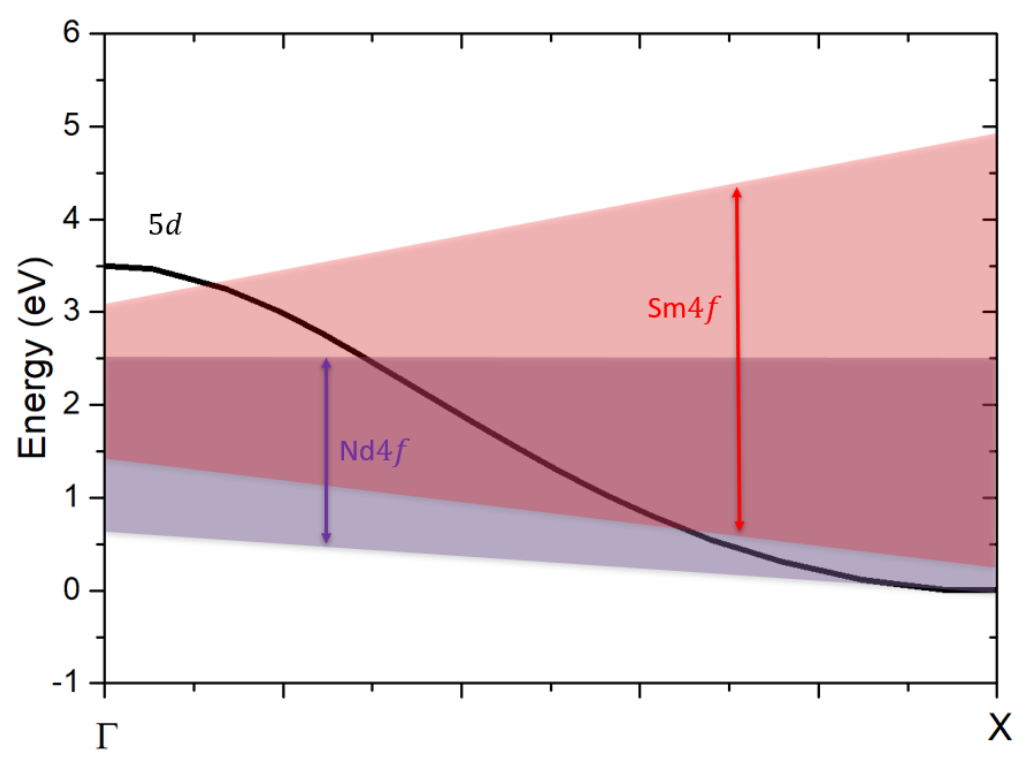

Figure 5.1: Schematic band structure diagram showing the range of calculated locations of lowest unoccupied Sm $4 f$ majority spin (red) and $\mathrm{Nd} 4 f$ majority spin (blue) bands, along with the $5 d$ band of $\mathrm{NdN}$ for reference (solid black). Plots are scaled such that zero energy is taken at the CB minimum. Data reproduced from Refs $[2,29,30,37]$.

channel, implying that the $4 f$ band does indeed exist near, if not form, the conduction band minimum. Nearly all calculations also result in a semimetallic ground state, in stark contrast to recent experimental reports, all of which support the semiconducting conclusion [1, 11, 52, 65, 58, 59]. It is clearly important to establish a band structure for $\mathrm{SmN}$ and determine the role played by the $4 f$ band.

The unfilled $4 f$ levels of NdN are also of interest, as has been seen in Chapter 4. The only calculation of the band structure proposes three solutions, largely differing on the location of the $4 f$ levels and their hybridisation with the $5 d$, schematically shown in Figure 5.1. These range from a $4 f$ band some few eV above the valence band maximum at $\Gamma$ with a $5 d$ conduction band minimum at $\mathrm{X}$, to the $4 f$ band forming the minimum optical bandgap at $\Gamma$ before hybridising with the $5 d$ band near $X$, both compris- 
ing the conduction band minimum [2]. Similar to $\mathrm{SmN}$, measurements of the anomalous Hall effect in NdN again show some influence of the $4 f$ band on the conduction channel. A thorough investigation of $\mathrm{NdN}$ in the mid / near infra-red and visible regions may be useful in locating these unfilled majority spin $4 f$ states and helping to guide future calculations.

\subsection{Experimental Techniques}

Before discussing the details of the measurement and modelling process, it is useful to have the full picture in mind. This process is relatively involved due to the requirement of separating the final measurements into contributions from the substrate, capping layer and rare earth nitride film. The process is completed in several stages

1. Measurement and modelling of reflection and transmission spectra of clean substrate materials.

These substrates are $0.5 \times 5 \times 10 \mathrm{~mm}^{3} \mathrm{Si}$ and sapphire.

2. Measurement and modelling of reflection and transmission spectra of each substrate with a deposited AlN capping layer.

An independent model for the AlN capping layer can be made.

3. Measurement and modelling of reflection and transmission spectra for a full sample comprising a rare earth nitride layer capped with AlN on each substrate material.

As models for the two substrates and capping layer exist the rare earth nitride can be modelled independently.

\subsubsection{Measurement and Data Processing}

The basic concept of the optical spectroscopy used in the present study is to measure the intensity of transmitted and reflected light from a mate- 
rial as a function of the energy of the incident light. Such a study can be achieved in several ways. The most intuitive is dispersive spectroscopy. Here the material is illuminated with a monochromatic light source, then the absolute transmission and reflection are measured as a function of energy. The technique used in this study, which has several advantages over a dispersive technique, is interferometry which is briefly described below.

An interferometer uses a broad spectrum light which is passed from the source through a beam splitter where it is then directed towards one fixed and one moving mirror. The light is reflected from each mirror then recombined at the beam splitter and directed towards the sample, then reflection and transmission spectra are measured. These spectra are measured not as a function of energy but rather the optical path difference $x$ created by altering the position of the moving mirror. As $x$ is varied the two beams interfere with each other when recombined at the beam splitter. The interference is due to the path difference caused by the moving mirror and changes the intensity of the light incident on the sample for any given path difference $x$. The maximum intensity being at $x=0$ or no optical path difference. Plots of the intensity as a function of path difference $x[\mathrm{~cm}]$ are called interferograms. The Fourier transform of the interferogram gives the intensity as a function of wavenumber $\left[\mathrm{cm}^{-1}\right]$, the desired measurement.

It is instructive to first consider the interferogram of a monochromatic source; this will be a simple cosine function whose Fourier transform will be a delta function centred at the frequency of the source. As more frequency content is added to the source, the interferogram will become more complex, and the Fourier transform will now show this increased frequency content. When using a real broad spectrum source, the frequency content will be continuous. An example of this is given in Figure 5.2. The top left-hand panel shows an artificially generated interferogram from a single frequency source, below is the Fourier transform with a single peak. The central panel shows another artificial interferogram, now with 

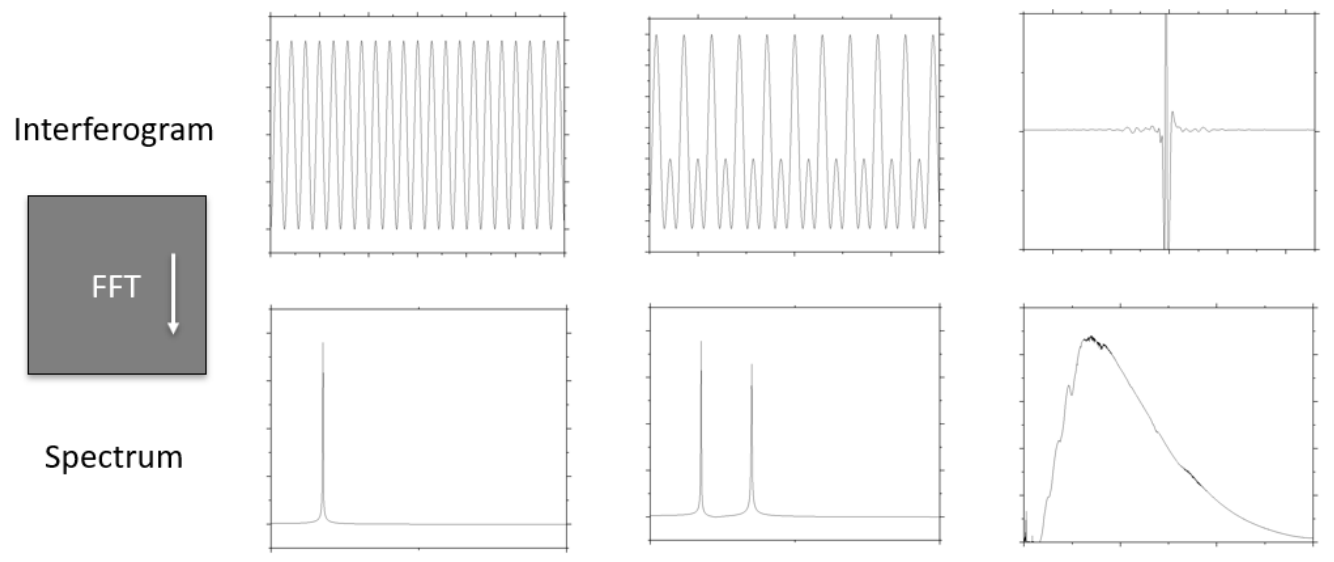

Figure 5.2: The top panels show three interferograms, two artificial and one experimental. The $x$-axes are optical path difference $(\mathrm{cm})$ and $y$-axes are intensity. The bottom three panels shows the Fourier transform of each interferogram, with axes of intensity and wavenumber $\left(\mathrm{cm}^{-1}\right)$

two frequencies, the Fourier transform shows two peaks. The final panel shows a measured interferogram for a mid infra-red light source. This has much more complex behaviour that can't be appreciated on the scale of the Figure. The Fourier transform of this is now a continuous spectrum.

Due to the nature of the measurement the resolution of the spectrum is limited by the maximum optical path difference $x$, created by the moving mirror. For the case of the Bruker Vertex 80v used in this study $x_{\max } \approx 16 \mathrm{~cm}$, so the spectral resolution is $0.06 \mathrm{~cm}^{-1}$ or $0.025 \mathrm{meV}$, however, this resolution is not required for the present study, which ran at a maximum resolution of $\sim 4 \mathrm{~cm}^{-1}$ or $\sim 0.5 \mathrm{meV}$.

Fourier transform interferometers bring with them several advantages. As measurements are conducted over the entire energy range at once, the signal to noise for a measurement over a given energy range in a given time is far greater than a dispersive spectrometer. The use of light of a range of frequencies also removes monochromators from the optical path, which would generally reduce the intensity, again leading to an increase in noise in a given measurement time. We now discuss the specific mea- 


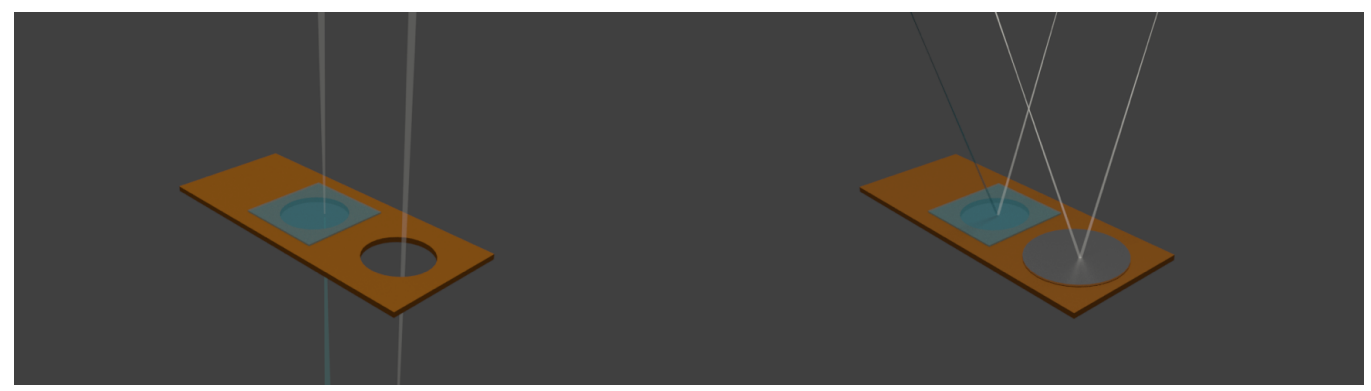

Figure 5.3: Measurements were conducted by mounting the sample on a copper stage inside a LHe flow through cryostat. Transmission measurements (left) were conducted by first measuring the background spectra through a hole in the stage and comparing this to the measurement of the sample. Reflectivity measurements (right) were conducted similarly but with an $\mathrm{Al}$ film in the place of the hole to serve as a background.

surement techniques used in this study.

\section{Transmission Measurements}

Measurements were conducted by mounting a sample on a copper stage covering one of two openings, as shown schematically in Figure 5.3. The stage is mounted in the interferometer and rotated to maximise the reflected intensity from light falling on the surface, to ensure correct alignment. The height of the sample stage is also adjusted to maximise transmission and ensure the beam is striking the sample alone and not impinging on the copper stage. There are several aperture sizes available, and it is desirable to use the largest spot size possible. This is limited by the mounting procedure and alignment in the system, generally a $2 \mathrm{~mm}$ spot size (at the sample location) is used.

As shown in Figure 5.3 there are two holes in the stage, one for the sample and a second, which allows for light over the spectral range of interest to first be collected as a background measurement. The collection of a reliable background signal is important as the various detectors and 
sources used have varying sensitivity and intensity over different energy regions. This sensitivity and structure must be carefully accounted for, and not attributed to the sample itself. Once a background spectrum has been collected, the sample is moved into the beam. The sample is moved using a stepper motor to control the vertical position of the stage. The transmission spectrum of the sample is then recorded over the same range as the background. The ratio of these measurements is then taken as the measured sample transmission. This process is repeated, and the results averaged to reduce noise. As some of these measurements are by necessity conducted on the time scale of hours, care is taken to ensure the sample is stable. An example of the background spectrum, sample spectrum and ratio is shown in Figure 5.4. Figure 5.4 shows a Measurement of a $\sim 150 \mathrm{~nm}$ AlN layer grown on a $0.5 \mathrm{~mm}$ Si substrate.

The top panel of Figure 5.4 shows the measured transmission of the background (black) and the sample (red) normalised to the maximum of the background signal. The bottom panel shows the final result, the ratio of two spectra. Significant structure can be seen in both spectra in the top panel, which is mostly absent in the final spectrum. Both the background and sample signals can be seen decreasing quickly at lower energy where the optical elements (detector, beam-splitter, etc.) are reaching their sensitivity limits. Increased noise levels can be seen in the ratio in the bottom panel as these signals both approach zero. At higher energy in the upper panel near $700 \mathrm{~cm}^{-1}$ both the background and sample signals can be seen to drop to zero, this is caused by strong absorption in the optical path, not related to the sample, and causes the large values in the lower panel at the same energy. The identification of regions where the background and sample spectrum are very small or rapidly changing is important so these regions can be treated and interpreted carefully in the final result.

The spectrum in the bottom panel of Figure 5.4 provides an excellent example of why Si substrates are used for investigation of the low energy far infra-red region. The very flat transmission in the region below 

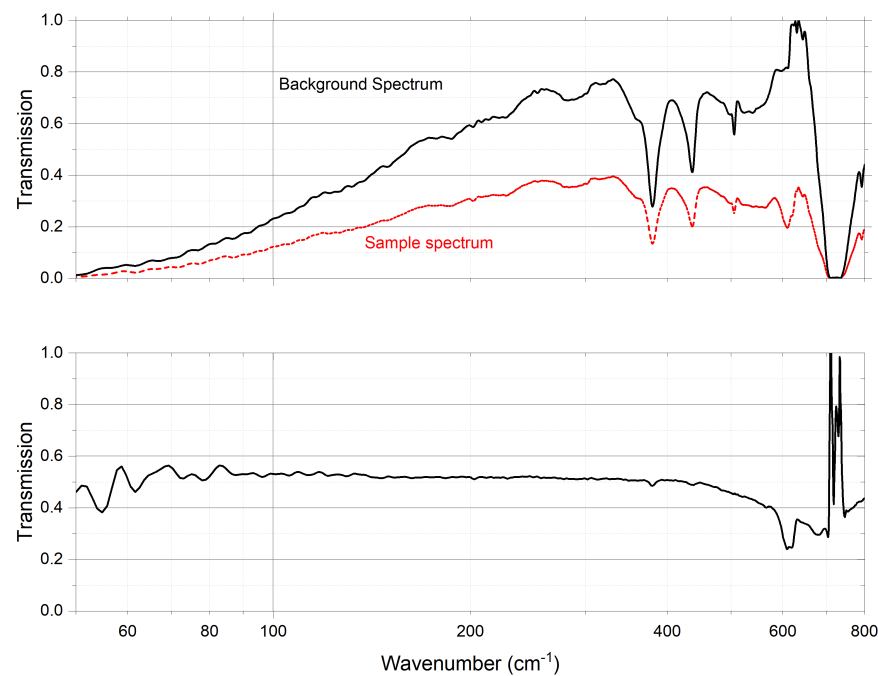

Figure 5.4: Measurement of transmission as a function of wavenumber for a $\sim 150 \mathrm{~nm}$ AlN film on a $0.5 \mathrm{~mm}$ Si substrate. A measurement consists of measuring a background transmission signal then the transmission signal through the sample (top panel). The ratio of these is then taken as the measured sample transmission (bottom panel). Si has a very flat response in the far infra-red region below $\sim 500 \mathrm{~cm}^{-1}$ so provides an ideal substrate for investigating the low energy region.

$500 \mathrm{~cm}^{-1}$ provides an ideal background for the measurement of phonon modes in the rare earth nitrides near $250 \mathrm{~cm}^{-1}$ [97], and free-carrier absorption at the lowest energies. The reduction in transmission and structure above $500 \mathrm{~cm}^{-1}$ in the top panel are caused by absorption in the Si and AlN layers.

\section{Reflection Measurements}

The process for measuring the reflectivity of a sample is similar, although now an Al mirror is used to collect a background measurement. The sample is mounted on the copper stage in the same manner, however, instead of a hole to measure the background signal a glass slide with a $\sim 250 \mathrm{~nm}$ 
- $400 \mathrm{~nm} \mathrm{Al} \mathrm{film} \mathrm{is} \mathrm{mounted} \mathrm{to} \mathrm{measure} \mathrm{the} \mathrm{reflected} \mathrm{background} \mathrm{signal,}$ this is shown schematically in Figure 5.3. A laser is used to align the $\mathrm{Al}$ mirror to be roughly co-planar to the sample. The mirror is adjusted until reflections from the sample and mirror are within $\sim 1 \mathrm{~cm}$ over a path difference of $3 \mathrm{~m}$, meaning the $\mathrm{Al}$ mirror and sample are co-planar within $\sim 0.2^{\circ}$. Once the $\mathrm{Al}$ mirror and sample are aligned, the stage is inserted into the interferometer. The signal reflected from the $\mathrm{Al}$ mirror is maximised to ensure the beam is aligned. The reflectivity data are then collected, as described for the transmission above.

$\mathrm{Al}$ is chosen as a reference material for the reflectivity measurement as it is close to $100 \%$ reflective over most of the range of interest. A simple understanding of this is given by the plasma reflectivity, Al having a plasma frequency above $\sim 10 \mathrm{eV}$. The reflectivity of $\mathrm{Al}$ does, however, have some structure below the ultraviolet region, in particular a dip near $12,000 \mathrm{~cm}^{-1}$ $(\sim 1.5 \mathrm{eV})$ due to interband absorption. This must be accounted for to accurately measure the sample reflectivity. This is achieved by adjusting the measured sample reflectivity by the reflectivity of $\mathrm{Al}$ taken from literature [98]. An example of this process on a sapphire sample is given in Figure 5.5. The reflectivity of the sample is shown before and after adjustment (red) along with the reflectivity of $\mathrm{Al}$ (black)[98]. The dip in the $\mathrm{Al}$ reflectivity near $12,000 \mathrm{~cm}^{-1}$ can be seen to cause an increase in the measurement in this region, this is then removed in the adjusted spectrum. The reflectivity of sapphire is expected to be relativity flat through the visible and ultraviolet regions, the decrease at low energy corresponds to a vibrational mode near $500 \mathrm{~cm}^{-1}$ in the sapphire.

In addition to the recognised dip in the reflectivity of any Al mirror, the finite reflectivity of the mirror in question must be considered. Although the absolute reflectivity of the mirror in question is not known a first consideration here, which can at least be estimated, is the skin effect. The skin depth $\delta$ in a material where the conductivity is approximately constant with frequency, can be written as 


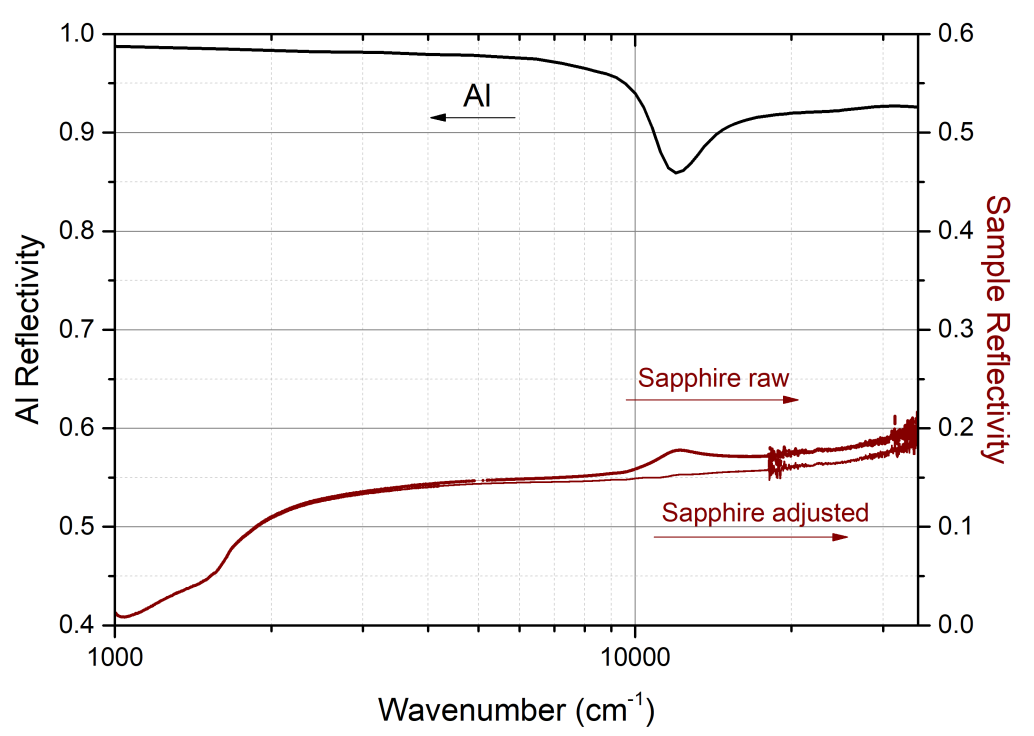

Figure 5.5: Raw and adjusted reflectivity of a sapphire substrate (red) along with the reflectivity of $\mathrm{Al}$ taken from literature [98]. The finite reflectivity of the $\mathrm{Al} \mathrm{mir-}$ ror used as a reference affects the sapphire spectrum and needs to be accounted for.

$$
\delta=\sqrt{\frac{2 \rho}{\omega \mu_{0} \mu_{r}}}
$$

where $\rho$ is the resistivity of the conductor, $\omega$ is the frequency of the electric field and $\mu_{0}, \mu_{r}$ are the vacuum and relative permittivity respectively. If this is evaluated in the far infra-red region between $50 \mathrm{~cm}^{-1}$ and $100 \mathrm{~cm}^{-1}$ for $\mathrm{Al}$ one finds the skin depth on the order of $50 \mathrm{~nm}$. The $\mathrm{Al}$ reflectors used in this study have a nominal thickness of $250 \mathrm{~nm}-400 \mathrm{~nm}$, thus one would assume roughly $2.5 \%$ transmission at the lowest energies, dropping to $0.5 \%$ by $100 \mathrm{~cm}^{-1}$ for the thinnest mirrors. This may seem like a negligible contribution however for the case of a semiconducting material with a very low carrier concentration the absorption in this region should be close to zero, thus even a small error here is noticeable. Due to this, the transmission through the thinnest $(250 \mathrm{~nm}) \mathrm{Al}$ mirror was measured in the far infra-red region, similarly to the sample described above. This 
measurement of transmission was then used to adjust the measured sample reflectivity (for measurements using the thinner mirrors) in addition to the literature values previously discussed.

\section{Temperature Dependant Measurements}

The sample stage previously discussed is located inside a liquid He cooled flow through cryostat. Measurements as a function of temperature down to $\sim 5 \mathrm{~K}$ were conducted by cooling the sample and controlling the temperature using a Lake-shore 331 temperature controller. When running routine room temperature measurements, the sample and spectrometer are kept under a rough vacuum of $\sim 1 \mathrm{mb}$. Running at low temperatures required the sample and stage to be further isolated from the environment which required the installation of two $25 \mu \mathrm{m}$ polypropylene windows. With windows installed the sample space could be pumped to pressures of $\sim 1 \times 10^{-5} \mathrm{mb}$. These windows were removed for routine ambient temperature measurements as they absorb strongly at various frequencies.

\section{Data Processing}

The optical study detailed in this thesis covers not only the far infra-red region pictured in Figure 5.4 but spans the infra-red, visible and ultraviolet regions of the spectrum. This is far larger than the range of a single detector, and in fact five distinct optical setups are used. These are detailed in Table 5.1.

The decision of where to join two regions which are covered by two sets of measurements becomes important. Figure 5.6 shows the far infrared (red) and mid infra-red (orange) spectra for the AlN capped Si sample discussed above. The top panel again shows the background and sample spectra and the bottom panel shows the ratio. Looking at the top panel, the normalised intensity of the background measurements can be seen to be equal near $680 \mathrm{~cm}^{-1}$, which may indicate this is an appropriate point to 


\begin{tabular}{|c|c|c|c|c|}
\hline Range & Approx. Energy $(\mathrm{eV})$ & Source & Detector & Beamsplitter \\
\hline FIR & $0.012-0.075$ & Hg Lamp & DLaTGS & $6 \mu \mathrm{m}$ Mylar \\
\hline MIR & $0.06-0.62$ & Globar & DLaTGS & $\mathrm{KBr}$ \\
\hline NIR & $0.38-1.5$ & W Lamp & InGaAs & $\mathrm{CaF}_{2}$ \\
\hline VIS & $1.25-2.25$ & W Lamp & Si Diode & $\mathrm{CaF}_{2}$ \\
\hline UV & $2-4$ & Dueturum lamp & GaP & $\mathrm{CaF}_{2}$ \\
\hline
\end{tabular}

Table 5.1: Optical components used in various energy ranges.

splice the measurements together. This point, however, occurs at a sharp decrease in the sensitivity of the far infra-red detector. As the intensity of background and sample are both changing very quickly, a more appropriate point may be at lower energy near $550 \mathrm{~cm}^{-1}$. Although the mid infra-red detector is less sensitive here the background spectra of both are fairly featureless, making this a good location to join spectra. Each of the four splicing locations,

$$
\mathrm{FIR} \leftrightarrow \mathrm{MIR} \leftrightarrow \mathrm{NIR} \leftrightarrow \mathrm{VIS} \leftrightarrow \mathrm{UV}
$$

must be joined in such a fashion to create a continuous spectrum.

As different detectors are used for each region, the optical path for individual measurements is slightly different, causing different amplitudes in the final measurement. When the spectrometer is aligned correctly, differences in amplitude are less than $2 \%$. Small offsets are applied at the splice locations to account for this. This process is repeated for each measured spectrum for both reflection and transmission to produce the final reflection and transmission measurements over the entire range.

Once reflection and transmission spectra are collected a simple view of the absorption (1-R-T) can be calculated. This is shown in Figure 5.7 for the Si substrate capped with $\sim 150 \mathrm{~nm}$ of AlN discussed above. Figure 5.7 shows the transmission in red, reflection in blue and the absorption (1$\mathrm{R}-\mathrm{T}$ ) in black. The main contribution to the absorption is centred around 

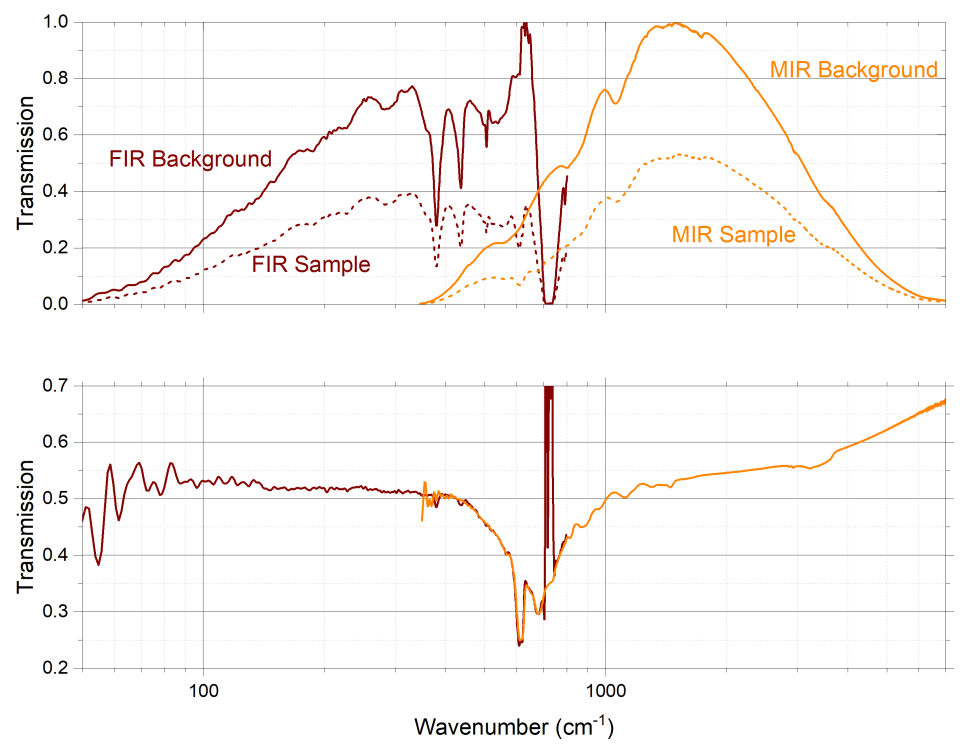

Figure 5.6: Measurement of transmission as a function of wavenumber for a $\sim 150 \mathrm{~nm}$ AlN film on a $0.5 \mathrm{~mm}$ Si substrate, data here are collected in both the far infra-red (red) and mid infra-red (orange) regions. The top panel shows the background and sample transmission, the bottom panel shows the ratio. Spectra in the bottom panel can be joined at a location informed by the top panel.

$\sim 500 \mathrm{~cm}^{-1}$, this is caused by absorption in the Si and AlN capping layer. We see another discrete contribution near $3000 \mathrm{~cm}^{-1}$ caused by $\mathrm{OH}$ vibrations, likely in the capping layer. The absorption then rises sharply near $10,000 \mathrm{~cm}^{-1}$ as we reach the bandgap of Si. Apart from these noted features, the absorption is largely flat, making Si a suitable substrate for investigating the low energy properties of the rare earth nitrides.

To study the properties of rare earth nitride films over the entire energy range from $\sim$ meV to $5 \mathrm{eV}$, films must be grown simultaneously on both $\mathrm{Si}$ and sapphire substrates. Measurements of reflection and transmission are then made on each substrate material. These are joined in the largely transparent region near $5000 \mathrm{~cm}^{-1}$ shared by both substrate materials. This is shown in Figure 5.8, which in the top panels shows the measured reflection, transmission and calculated absorption for Si (left) and sapphire 


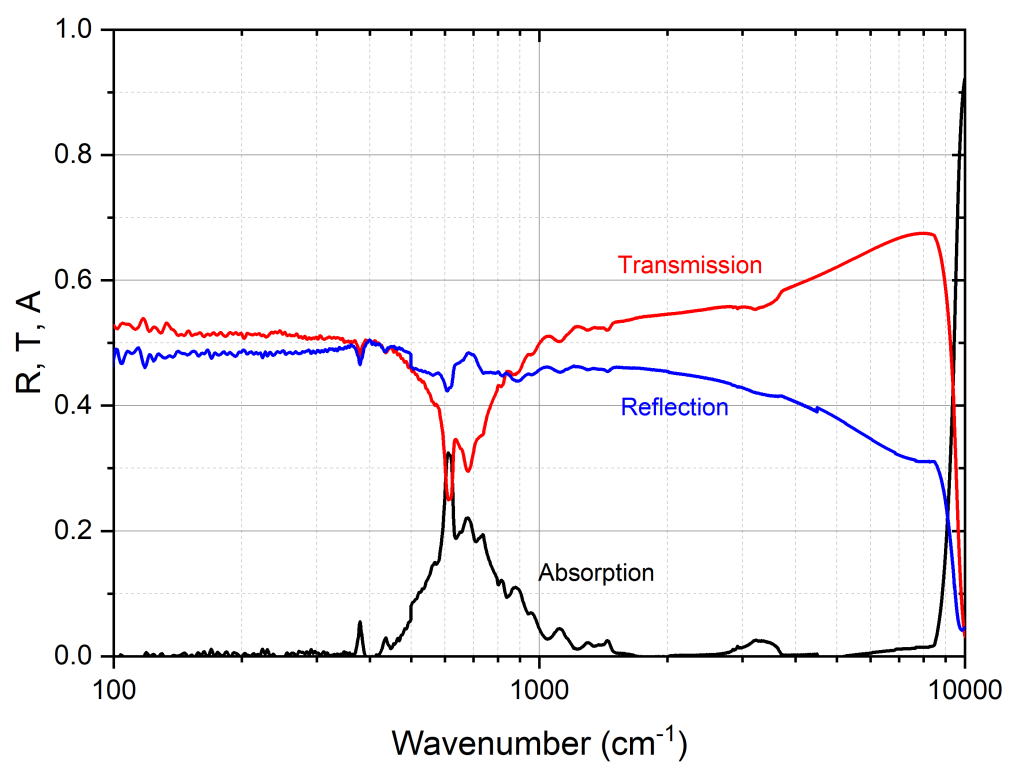

Figure 5.7: Measurements of reflection and transmission and the calculated absorption as a function of wavenumber for a Si substrate with $\sim 150 \mathrm{~nm}$ AlN capping layer. Absorption can be seen near $500 \mathrm{~cm}^{-1}$ caused by absorption in the $\mathrm{Si}$ and AlN. Strong absorption in the Si begins near $10,000 \mathrm{~cm}^{-1}$.

(right). The bottom panel shows the absorption for each substrate with the areas used for $\mathrm{Si}$, sapphire and the mixed region identified.

\subsection{Reproducing Optical Measurements Using Ref- Fit}

RefFit [99] is freely available software which can be used to reproduce measured optical properties, such as the reflection and transmission of a material as a function of energy. Based on the classical oscillator model [54], described briefly in Section 2.2.2, RefFit uses an inherently Kramers-Kronig consistent sum of Lorentzians [100] to represent the complex dielectric function $\epsilon(\omega)$ of a material 

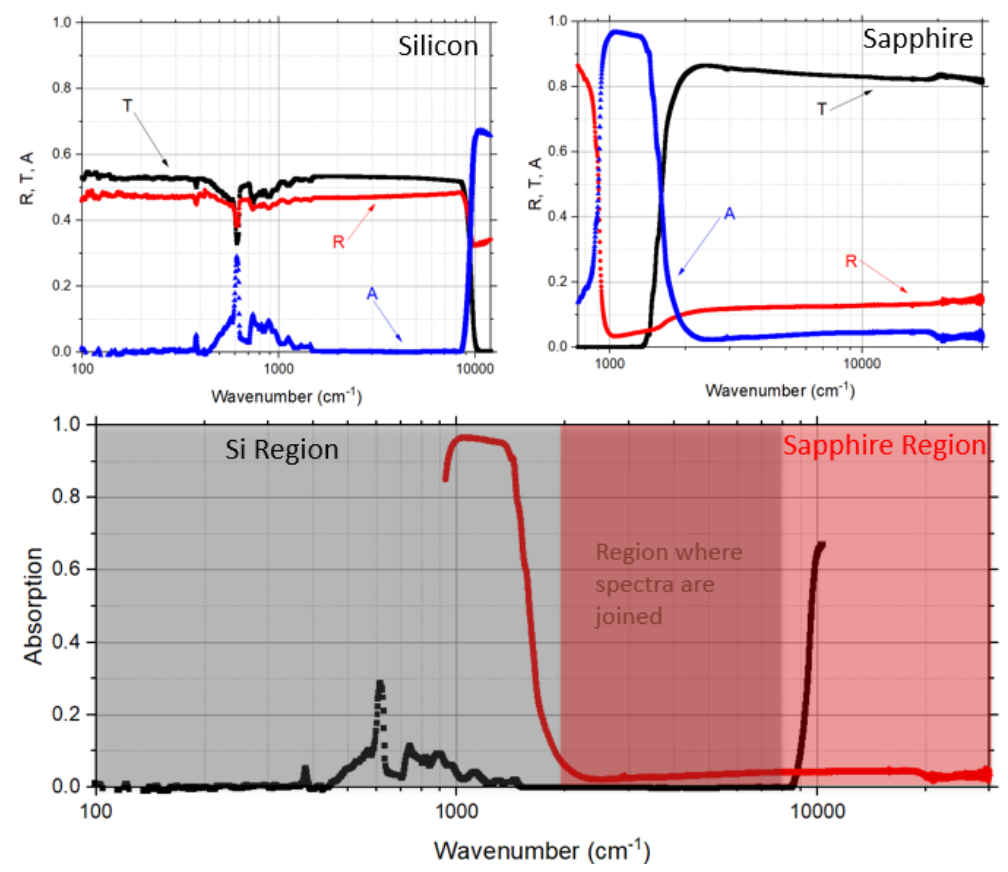

Figure 5.8: Measurements of reflection and transmission and the calculated absorption on Si (top left) and sapphire (top right) substrates. The bottom panel shows the regions where each substrate is used for the final measurement.

$$
\epsilon(\omega)=\epsilon_{\infty}+\sum_{j} \omega_{p_{j}}^{2} \frac{1}{\left(\omega_{0 j}^{2}-\omega^{2}-i \gamma_{j} \omega\right)}
$$

where $\omega_{p}$ is the plasma frequency, $\omega_{0}$ is the transverse or resonant frequency, $\gamma$ is the scattering rate and $\epsilon_{\infty}$ represents all high energy contributions to $\epsilon(\omega)$. The Fresnel equations [101] are then used to model the behaviours of a multilayer system. These equations approximate the interfaces between materials as perfectly flat with refractive indices which change discontinuously at the boundaries.

Although reflection or transmission measurements alone are in principle enough to determine the dielectric function of a material, measurements of both were conducted. Reflection and transmission measurements were then reproduced simultaneously for greater assurance that the data 


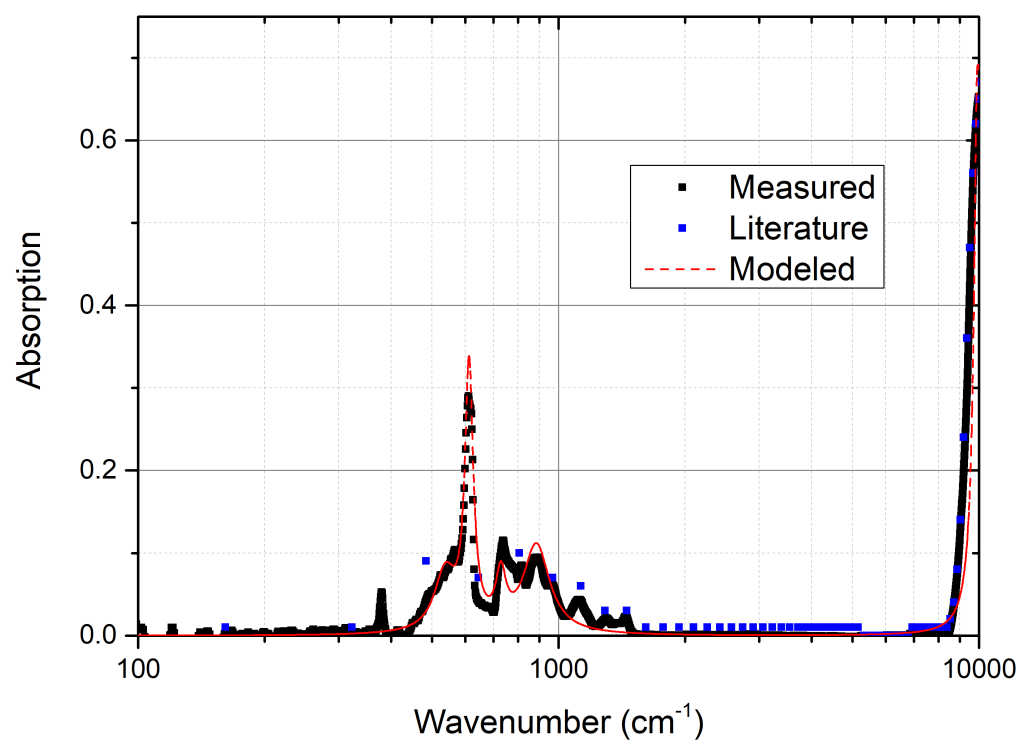

Figure 5.9: Measurements of reflection and transmission of a $0.5 \mathrm{~mm} \mathrm{Si}$ substrate have been used to determine the absorption (1-R-T), these are shown along with the model produced in RefFit and values for the absorption of Si taken from literature [102].

collected and model created accurately represents the material.

When modelling the $\sim 0.5 \mathrm{~mm}$ thick substrates reflections were determined to be incoherent, i.e. the phase relationship is lost after multiple reflections. Models for the Si and sapphire substrates used were constructed by adding Lorentzians with varying $\omega_{p}, \omega_{0}$ and $\gamma$ to simultaneously recreate both the measured transmission and reflection spectra. The refractive index from the models were found to be consistent with literature values $[103,104]$. As an example the measured, modelled and literature spectra [102] for the absorption of $\mathrm{Si}$ is shown in Figure 5.9.

As the rare earth nitrides also require a capping layer to be grown on top of the film, the optical properties of this had also to be determined. AlN capping layers were used for all optical measurements. AlN films of $\sim 100 \mathrm{~nm}$ were grown simultaneously on both Si and sapphire substrates. These were first measured as described above then modelled. There now 


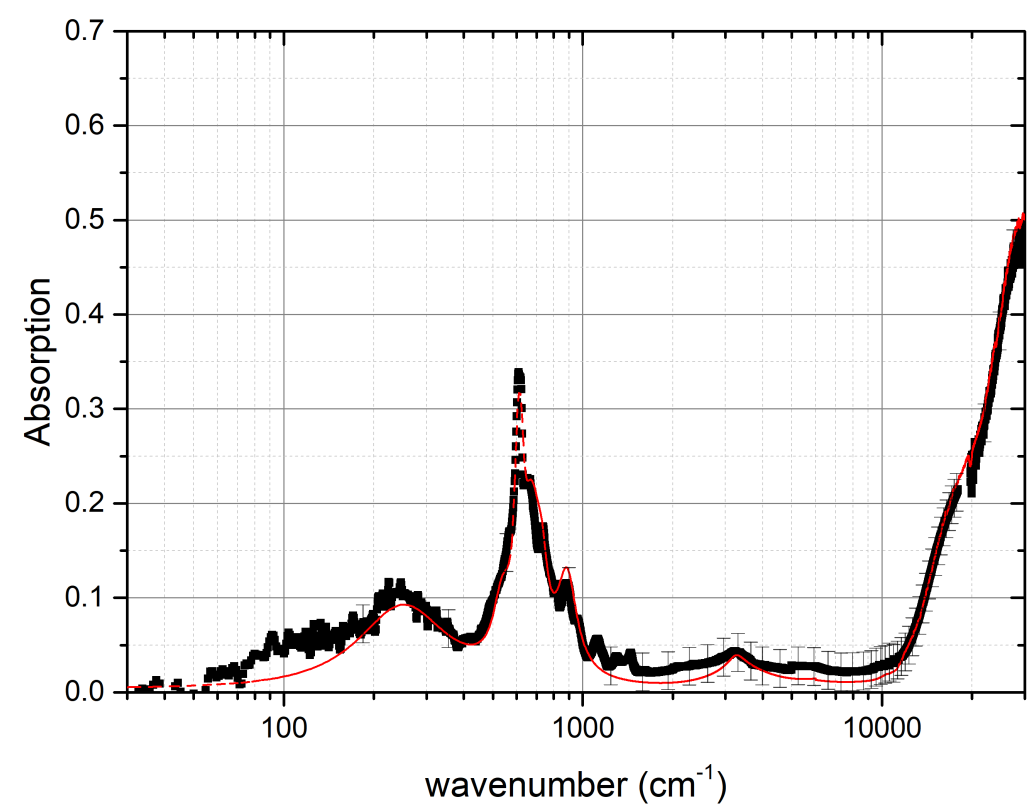

Figure 5.10: Measurement of the absorption in a GdN film (A543) capped with AlN and grown simultaneously on Si and sapphire substrates. Measurements of the absorption are constructed from low energy measurements on Si and high energy measurements on sapphire to create a full spectrum absorption of the sample. A model based on the separate layers has been plotted in red, from this the complex dielectric function $\epsilon(\omega)$ of the $\mathrm{GdN}$ layer alone can be extracted.

exist four separate measurements of the AlN films, reflection and transmission on both Si and sapphire. These were modelled simultaneously, and this now consisted of four, rather than the two spectra for the substrate materials. A refractive index value in line with literature was again found for the measured AlN [105].

Measurements on complete samples were then conducted and by applying the models previously created for the substrates and capping layers, the contribution of the rare earth nitride layer alone could be determined. When modelling the rare earth nitride layer, ideally an infinite number of Lorentzians should be used to represent the continuous function $\epsilon(\omega)$. This was not possible, so an array of Lorentzians spaced every 
$100 \mathrm{~cm}^{-1}$ with width of $\gamma=100 \mathrm{~cm}^{-1}$ from $\sim 300 \mathrm{~cm}^{-1}$ to $40,000 \mathrm{~cm}^{-1}$, roughly 400 discrete functions, was instead used. The amplitude $\omega_{p}$ for each Lorentzian was then manually adjusted to simultaneously match the transmission and reflection measurements on both $\mathrm{Si}$ and sapphire substrates. In this way each model was consistent with four separate measurements. In the low energy range a single term $\left(\omega_{0}=0\right)$ was used, consistent with the simple Drude theory, to model the zero frequency behaviour of the film. Around $250 \mathrm{~cm}^{-1}$ a single Lorentzian with variable $\omega_{p}, \omega_{0}$ and $\gamma$ was used to model phonon absorption.

Figure 5.10 shows an example of a fully treated measurement of a GdN film grown simultaneously on sapphire and Si substrates and capped with AlN. Reflection and transmission data have been collected on Si samples at low energy from $\sim 30 \mathrm{~cm}^{-1}$ to $10,000 \mathrm{~cm}^{-1}$ and on sapphire from $\sim 1000 \mathrm{~cm}^{-1}$ to $30,000 \mathrm{~cm}^{-1}$. The data have been used to calculate the absorption and then joined near $8000 \mathrm{~cm}^{-1}$, where both substrate materials are for the most part transparent. A model for the data was then created to simultaneously reproduce the four separate measurements over the two substrate materials. The model and data in Figure 5.10 show good agreement. The model can then be used to calculate the optical conductivity, refractive index and various other optical parameters across the full spectral range $\sim 50 \mathrm{~cm}^{-1}-30000 \mathrm{~cm}^{-1}$

\subsection{Results and Discussion}

\subsubsection{Samarium Nitride}

Thin films of SmN and GdN were grown at room temperature as described in section 3.1. The present films were grown at a high nitrogen pressure and low rare earth flux to limit nitrogen vacancies. Structural analysis for these films found both to be textured in the [111] direction with lattice parameters of $5.01 \AA$ and $5.07 \AA$ and crystallite sizes of $15.6 \mathrm{~nm}$ and $22.8 \mathrm{~nm}$ 

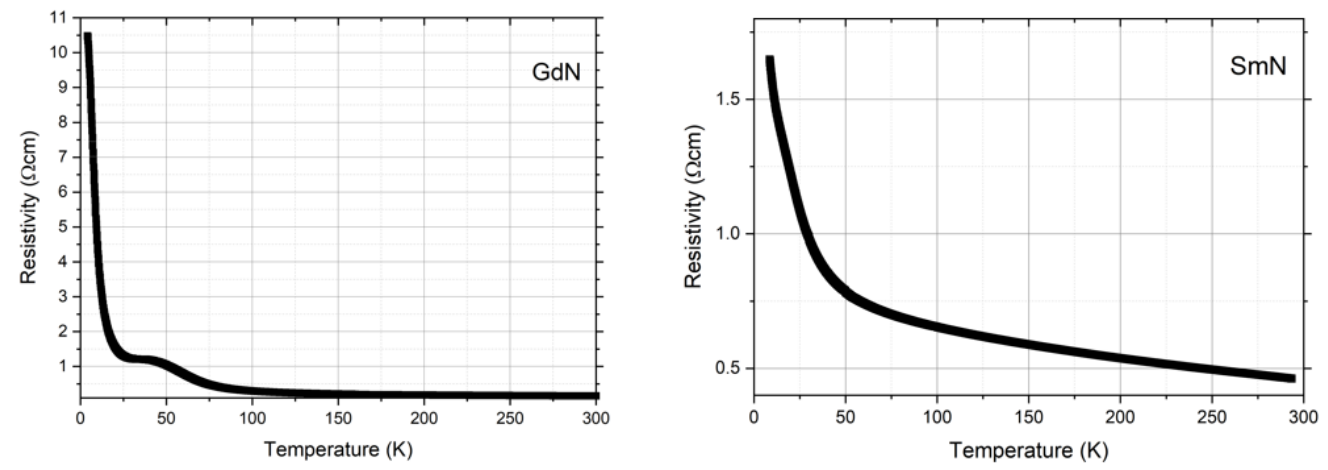

Figure 5.11: Resistivity as a function of temperature for the $\mathrm{SmN}$ and GdN films used for room temperature measurements. The resistivity of each can be seen to increase rapidly at low temperature, indicating a non-metallic ground state.

for the GdN and SmN films respectively. These are typical values for polycrystalline rare earth nitride films grown at room temperature. The non-metallic nature of these films was first identified via the temperaturedependent resistivity. Both films showed a fairly large resistivity at room temperature, which increased rapidly at low temperature indicating few occupied states at zero temperature. The temperature-dependent resistivity of each of these films is presented in Figure 5.11. An anomaly can be seen in the resistivity of SmN near $20 \mathrm{~K}$ and GdN near $50 \mathrm{~K}$ indicative of the magnetic transition in these materials.

Measurements (black) of the absorption (1-R-T) for the $\mathrm{SmN}$ (top) and GdN (bottom) films are shown in Figure 5.12 along with modelled spectra (red). These measurements are constructed from low energy measurements, in the far infra-red to near infra-red regions, of the samples on $\mathrm{Si}$ substrates and high energy measurements, from the near infra-red to ultraviolet regions, on sapphire. The two spectra are joined near $0.3 \mathrm{eV}$. The model for each spectrum can be seen to match the measurement well with slight differences at low energy. Above $\sim 1.3 \mathrm{eV}, \mathrm{GdN}$ and $\mathrm{SmN}$ show very similar absorption with differences in the magnitude and structure largely due to thickness and interference effects from the substrates and capping 


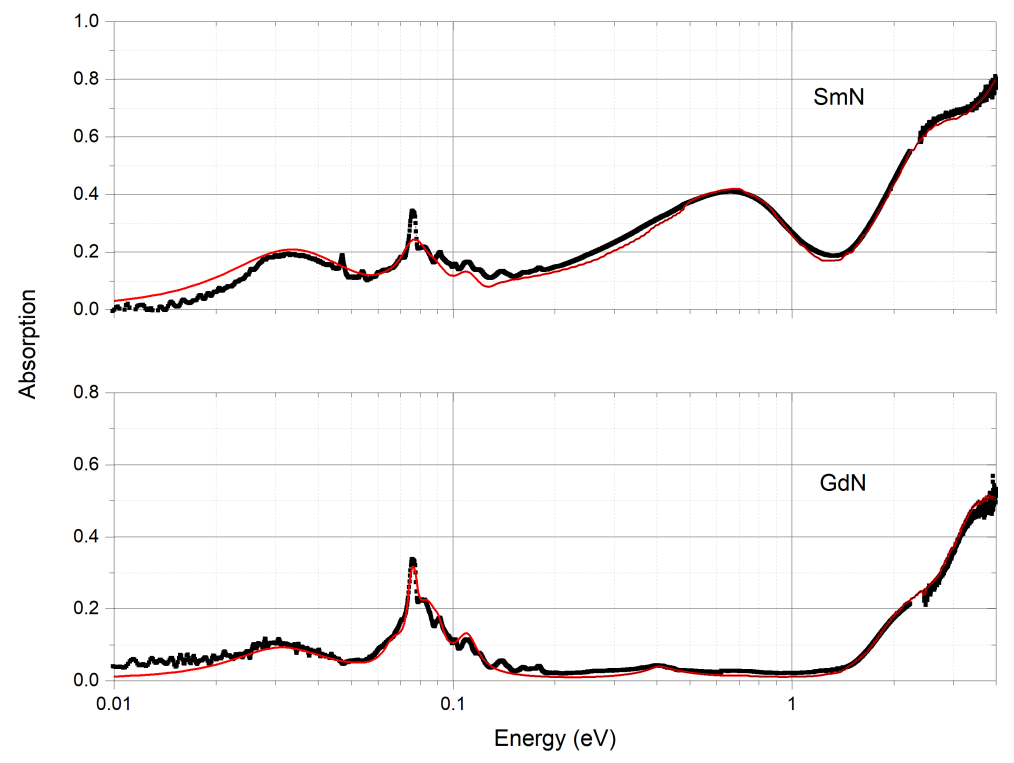

Figure 5.12: Absorption in $\mathrm{GdN}$ and $\mathrm{SmN}$ at ambient temperature. The GdN measurement shows little absorption below the fundamental edge at $1.35 \mathrm{eV}$. The $\mathrm{SmN}$ measurement shows a similar absorption above $1.2 \mathrm{eV}$ but an addition feature at lower energy signals optical transitions not present in GdN. The features near $0.1 \mathrm{eV}$ are caused by absorption in the substrate and capping layer. The broad absorption near $0.03 \mathrm{eV}$ is a phonon absorption in each rare earth nitride layer.

layers. Moving to the lower energy regions, GdN shows little absorption in the transparent region below the minimum optical gap of $\sim 1.3 \mathrm{eV}$. The small absorption present near $0.3 \mathrm{eV}$ is caused by an $\mathrm{OH}$ vibrational mode.

While GdN is largely free of absorption below the optical edge in the near infra-red region, $\mathrm{SmN}$ shows a strong absorption here. The most natural explanation for this additional absorption, based upon the electronic structure of these materials, is excitation from the valence band into the unfilled majority spin $4 f$ band of $\mathrm{SmN}$, not present in $\mathrm{GdN}$, and predicted to lie variously from the bottom of the conduction band to some few $\mathrm{eV}$ higher $[2,29,30,37]$. This is now discussed in more detail via the optical conductivity of each material. 


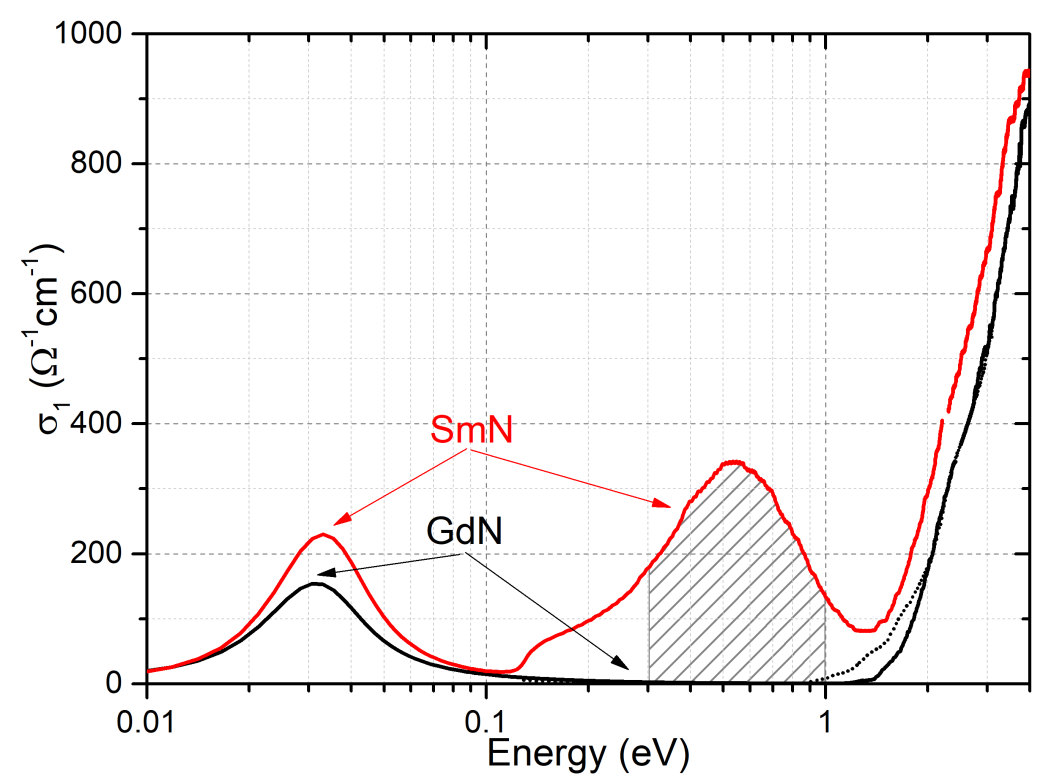

Figure 5.13: The real part of the optical conductivity $\sigma_{1}(\omega)$ based on data visible in Figure 5.12 for both $\mathrm{SmN}$ (red) and GdN (black). Both materials show a strong increase in $\sigma_{1}(\omega)$ at high energy due to transitions from the valence to $5 d$ bands. The $\mathrm{SmN}$ result has an additional feature near $0.5 \mathrm{eV}$ (identified with cross-hatching) which indicates transitions from the valence into a localised $4 f$ band, lying below the $5 d$. At the lowest energies a phonon absorption can be seen in each material.

The optical conductivities $\sigma_{1}(\omega)$ for GdN and SmN are shown in Figure 5.13. This plot shows similar features to the absorption but is now free from interference effects and contributions from the substrates and capping layers, thus represents the rare earth nitride layer alone. For the GdN sample $\sigma_{1}(\omega)$ increases strongly above $1.3 \mathrm{eV}$, signalling the onset of interband transitions. Extrapolation of the absorption above this edge leads to a bandgap of $\sim 1.35 \mathrm{eV}$ in the paramagnetic phase. Under $\sim 1 \mathrm{eV}$ $\sigma_{1}(\omega)$ falls to zero in the transparent region below the minimum optical bandgap.

The optical conductivity for the $\mathrm{SmN}$ sample is very similar to that of the $\mathrm{GdN}$ above $\sim 1.2 \mathrm{eV}$ with a transposition to low energy on the or- 
der of $0.3 \mathrm{eV}$. Extrapolation from this absorption edge gives a bandgap of $\sim 1.27 \mathrm{eV}$ in the paramagnetic phase. It should be noted that this bandgap is difficult to determine due to the overlap with the lower energy feature.

Moving on to the mid / near infra-red region spanning 0.1 to $1 \mathrm{eV}$. There is now no absorption in the GdN, the SmN sample clearly shows additional absorption as illustrated in Figure 5.13 by a broad peak in $\sigma_{1}(\omega)$ centred around $0.5 \mathrm{eV}$ (cross-hatched). This feature indicates not only absorption at lower energies than $\mathrm{GdN}$, but its form reveals information regarding the nature of the band, or density of states, at the bottom of the conduction band.

The task is now to construct a schematic band structure based on the measurements in Figure 5.13, and in the context of the calculated band structure of $\operatorname{GdN}[2,37,106]$, which is largely consistent with experiment $[24,60]$. The resulting schematic band structure of $\mathrm{SmN}$ is shown in Figure 5.14 .

To begin, the valence band must be considered, this is formed from the $\mathrm{N} 2 p$ states as is the case across the semiconducting members of the rare earth nitride series [2]. The valence band maximum is at $\Gamma$ and $\sim 0.5 \mathrm{eV}$ of dispersion before a stationary point at $\mathrm{X}$. The filled $4 f$ states, similar to $\mathrm{GdN}$, are calculated to be a minimum of $\sim 5 \mathrm{eV}$ below the conduction band so are of little influence on the energy scales probed in this study [2]. The $5 d$ band which forms the conduction band minimum in $\mathrm{GdN}$, is also qualitatively similar across the series. The data in Figure 5.13 show such similarity above $1.2 \mathrm{eV}$ that it is proposed the absorption here is due to the same $2 p$ to $5 d$ transition beginning at $\mathrm{X}$ that has been so well established in $\operatorname{GdN}[2,24,60,96]$. The feature near $0.5 \mathrm{eV}$, not present in the $\mathrm{GdN}$ data, must then be described by excitation into some state not present in $\mathrm{GdN}$. The most natural description is then the unfilled majority spin $4 f$ band of SmN implied by both anomalous Hall effect [59] and superconductivity [58] measurements to be near the conduction band minimum. In the limit of a dispersionless band, the separation of this feature in $\sigma_{1}(\omega)$ 
from the absorption at higher energies indicates that optical transitions begin at some point closer to $\Gamma$, as the energy of states in the valence band increases when moving from $X$ towards the valence band maximum at $\Gamma$ but decreases monotonicity moving from $X$ to $W$ or from $X$ to $K$ [2].

Furthermore, the form of the feature points towards transitions into a very flat band. This can be seen by noting that the optical conductivity $\sigma_{1}(\omega)$ is inversely proportional to the joint density of states $g(\hbar \omega)$. Strong contributions are made to the joint density of states and thus $\sigma_{1}(\omega)$ at regions near where the difference in energy between the valence bands and conduction bands $\left(E_{c}-E_{v}\right)$ is stationary, i.e. $\nabla_{k}\left(E_{c}-E_{v}\right)$ vanishes. The stationary points themselves mark van Hove singularities and can be identified as points where the conduction and valence bands are parallel. This is the case for both materials at the $\mathrm{X}$ point, but the discrete low energy feature in Figure 5.13 shows $\mathrm{SmN}$ must gain an additional stationary point, where $\nabla_{k}\left(E_{c}-E_{v}\right)=0$, between $\Gamma$ and $\mathrm{X}$. The dispersion of the $4 f$ conduction band here must then be less than that of the valence band, again indicating a flat or localised band.

Figure 5.14 shows a schematic representation of the band structure of $\mathrm{SmN}$ based on measurements in Figure 5.13. The $4 f$ band is shown with a range of energies for any given wave-vector $k$ (represented by the shading surrounding the band in the figure) due to its highly localised nature, especially in the case of minimal hybridisation shown. Figure 5.14 shows the $4 f$ band as dispersion-less, the width of the mid infra-red feature in Figure 5.13 is then the width of the valence band between $\Gamma$ and $\mathrm{X} \sim 0.5 \mathrm{eV}$ as qualitatively expected from calculations [2]. Extrapolation from the low energy side of the feature gives the energy of this band. Figure 5.14 shows the $1.27 \mathrm{eV}$ gap between the $\mathrm{N} 2 p$ valence band and Sm $5 d$ at X in black. The $\sim 0.3 \mathrm{eV}$ minimum optical gap is shown, in the limit of a dispersionless $4 f$ band, at the $\Gamma$ point.

Furthermore, we can use the schematic band structure shown in Figure 5.14 to complete a rough calculation of the form of the transition rate 


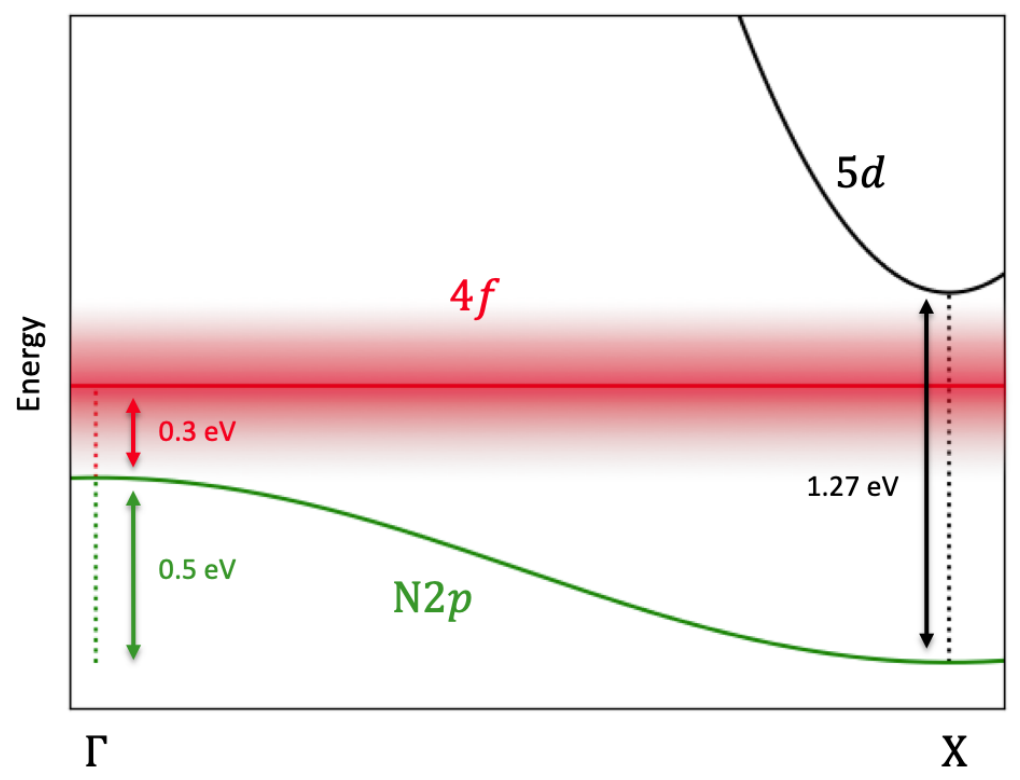

Figure 5.14: Schematic band structure for $\mathrm{SmN}$, which is consistent with the data in Figure 5.13. The Sm $5 d$ band, similar to that of $\mathrm{GdN}$, is shown reaching a minimum at the $\mathrm{X}$ point, the $\mathrm{N} 2 p$ valence band has its maximum at the $\Gamma$ point. The Sm $4 f$ band, located via measurements shown in Figure 5.13, is found beneath the $5 d$ band and is shown here as dispersion-less. Shading is used to represent this as a localised band which lacks a well defined $\epsilon(k)$ relationship.

$W_{i \rightarrow f}$ to test whether a qualitatively similar result is found to the measurement of $\sigma_{1}(\omega)$ shown in Figure 5.13. The density of states between $\Gamma$ and $X$ in the valence band was modelled as three parabolic regions evenly spaced from $k=0$ to $2 \pi$. The $5 d$ band was given parabolic dispersion and the $4 f$ band was dispersion-less. The calculation was completed in three dimensions for a band structure symmetric around the $X$ point. The resulting qualitative model of the transition rate is shown in the bottom panel of Figure 5.15, with the idealised band structure used for the calculation shown in the top panel. Although this is a very simplified model, a qualitatively similar result, including even the measured asymmetry, can be seen. The discrete low energy feature, shown in red, relates to transitions 


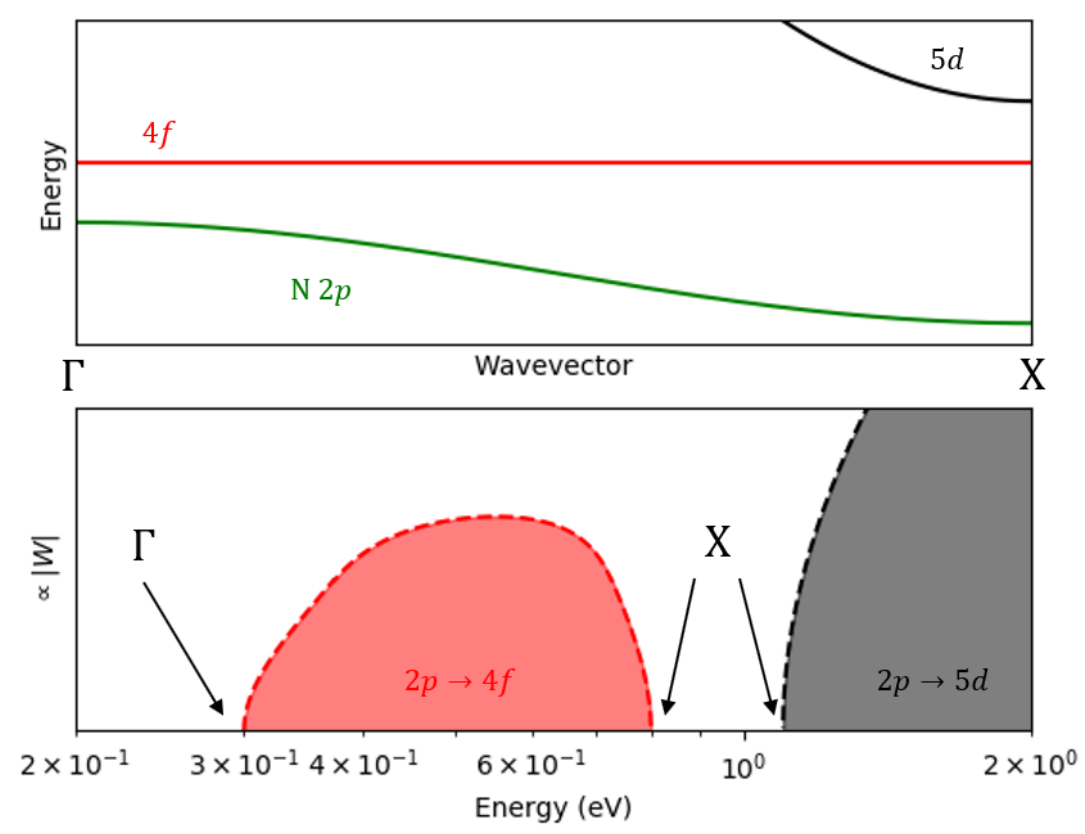

Figure 5.15: Top: Idealised band structure for SmN. Bottom: Simplified calculation of the optical transition rate $|W|$ based on the above band structure. The red feature near $0.5 \mathrm{eV}$ results from transitions from the valence band to the dispersion-less $4 f$ band, the grey feature results from transitions from the valence band to the $5 d$ band.

from the valence band to the flat $4 f$ band. The transition rate goes to zero, as indicated on the figure, at $\Gamma$ and $X$, where the density of states in the valence band approaches zero. The grey feature represents transitions from the $\mathrm{N} 2 p$ valence band to the $5 d$ band, which here is perfectly parabolic.

The band structure depicted in Figures 5.14 and 5.15 is not the only interpretation of the data, in fact various scenarios can be imagined. It may be the case that the $4 f$ band does not fall below the $5 d$ but instead hybridises resulting in an anti-crossing near $\mathrm{X}$. In this scenario the optical transitions near $0.5 \mathrm{eV}$ may now be from the valence band to the hybridised $4 f / 5 d$ conduction band minimum, or across the hybridisation gap itself.

At the lowest energy region, the far infra-red, Figure 5.13 show a phonon 
absorption in both GdN and $\mathrm{SmN}$ at $250 \mathrm{~cm}^{-1}(31 \mathrm{meV})$ and $265 \mathrm{~cm}^{-1}$ (33 meV) respectively. The measured values are $\sim 20 \%$ lower than those predicted [97] as is also seen in $\mathrm{DyN}$ [56] and in Raman measurements of several of the rare earth nitrides [97]. As energy approaches zero and the optical conductivity approaches the DC, there is no obvious contribution to $\sigma_{1}(\omega)$ indicative of absorption from free carriers. This indicates insulating films, consistent with transport measurements and previous experimental results, indicating a semiconducting ground state $[1,52,58,59,65]$. A zero frequency free carrier term was used to represent the lowest energy region where the scattering time $\tau$ is consistent with an electron whose mean free path is constrained by the lattice parameter and crystallite size, measured with XRD. The plasma frequency $\omega_{p}$ was chosen to be consistent with this scattering time and the measured DC conductivity. Using this, an upper limit can be put on the carrier concentration in these samples of $6 \times 10^{18} \mathrm{~cm}^{-3}$ and $1 \times 10^{18} \mathrm{~cm}^{-3}$ for the GdN and $\mathrm{SmN}$ samples respectively. The large resistivity of these undoped samples made Hall measurements impossible thus no comparison between the optical and Hall effect carrier concentrations and scattering times can be made.

\subsubsection{Neodymium Nitride}

Thin films of undoped $\mathrm{NdN}$ were grown on sapphire and Si substrates at ambient temperature as described in Section 3.1. Measurements of reflection and transmission were completed and modelled as above and can be seen in the top panel of Figure 5.16. The top panel of Figure 5.16 shows an absorption which is based on reflection and transmission measurements on both $\mathrm{Si}$ and sapphire substrates. The measurement is qualitatively similar to the GdN measurement shown in the bottom panel of Figure 5.12. At low energy a phonon absorption can be seen at $\sim 250 \mathrm{~cm}^{-1}$ essentially the same energy as the GdN phonon and some $15 \mathrm{~cm}^{-1}$ lower than $\mathrm{SmN}$ [21]. There is little to no absorption in the mid / near-infra-red regions below 

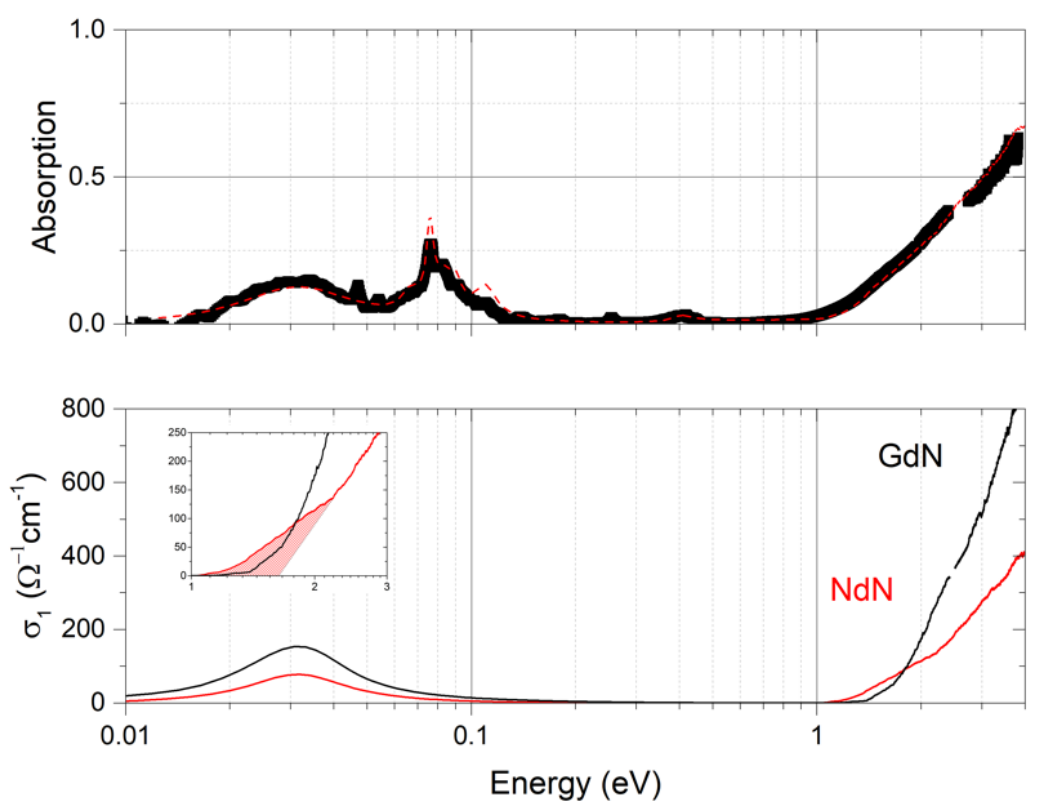

Figure 5.16: Top: Plot of absorption (1-R-T) as a function of energy for a $\mathrm{NdN}$ sample (A604). Phonon absorption in the $\mathrm{NdN}$ can be seen near $0.03 \mathrm{eV}$ $\left(250 \mathrm{~cm}^{-1}\right)$, the features near $0.8 \mathrm{eV}$ are caused by the Si substrate and AlN capping layer. Bottom: Plots of the optical conductivity of the $\mathrm{NdN}$ film, calculated from the above measurements, along with the optical conductivity of GdN for comparison (taken from Figure 5.13). The inset shows the area directly above the bandgap in each material with the additional contribution from the $\mathrm{Nd} 4 f$ band hatched.

the onset of absorption, seen as a gradual increase in $1-\mathrm{R}-\mathrm{T}$, at $\sim 1 \mathrm{eV}$. Absorption near $0.1 \mathrm{eV}$ is in the Si substrate and AlN capping layer, the small absorption near $0.3 \mathrm{eV}$ is an $\mathrm{OH}$ vibration.

The reflection and transmission data were modelled, as described in section 5.3. The result is shown as the red dashed series in the top panel of Figure 5.16 and matches the experimental data well. The optical conductivity $\sigma_{1}(\omega)$ was then determined and is shown in the bottom panel of Figure 5.16, as well as the optical conductivity of GdN (taken from Figure 5.13) for comparison. It is notable that the interband absorption above 
$2 \mathrm{eV}$ is a factor of $\sim 2$ weaker in $\mathrm{NdN}$ than in GdN. Such a contrast can be expected to follow from the increased lattice constant of $\mathrm{NdN}$, roughly $3 \%$ larger than GdN. The already small overlap between the N $2 p$ and $\mathrm{L} 4 f$ / $5 d$ wave functions in $\mathrm{GdN}$ is further reduced by the increased separation, leading to a decrease in the transition rate in the $\mathrm{NdN}$ data, and in turn the reduced optical conductivity in the interband region.

The GdN data in the lower panel of Figure 5.16 show a nearly featureless rise from the band edge to $\sim 4 \mathrm{eV}$. In contrast the NdN data appears to have two contributions with the initial absorption turning over above $1.5 \mathrm{eV}$ before an additional contribution to the absorption appears near $2.5 \mathrm{eV}$. The calculated band structure of GdN features the parabolic $5 d$ band alone $[2,96,106]$, which is matched well by experiments $[21,24,60$, $61,95]$. Calculations of the band structure of $\mathrm{NdN}$ show a similar $5 d$ band to $\mathrm{GdN}$, but in addition four unfilled majority spin $4 f$ bands. It is clear that any additional structure in the $\mathrm{NdN}$ data must be due to these $4 f$ bands not present in GdN and predicted to lie near the conduction band minimum in $\mathrm{NdN}$ [2]. The comparison between $\mathrm{NdN}$ and $\mathrm{GdN}$ can be seen more clearly in the inset of the lower panel of Figure 5.16, which shows $\sigma_{1}(\omega)$ for each in the region of the bandgap. The slightly convex form of $\sigma_{1}(\omega)$ between $1 \mathrm{eV}$ and $2.5 \mathrm{eV}$ in the $\mathrm{NdN}$ data is qualitatively expected from transitions into a flat band, as has been seen for $\mathrm{SmN}$ in the previous section.

These results need to be considered in the context of (i) the electrical transport measurements for $\mathrm{NdN}$ discussed in Section 4.4.2 which showed an enhanced anomalous Hall effect indicating a $4 f$ contribution to the conduction channel in an intentionally doped film, (ii) calculations [2] which place the lowest unoccupied majority spin $4 f$ band in $\mathrm{NdN}$ variously from the bottom of the conduction band to some few $\mathrm{eV}$ higher, and (iii) recent optical measurements on $\mathrm{SmN}$ [21] showing a similar low energy feature in the optical conductivity, identified as transitions into a $4 f$ band. The most natural description is then that the additional absorption in $\mathrm{NdN}$ is 


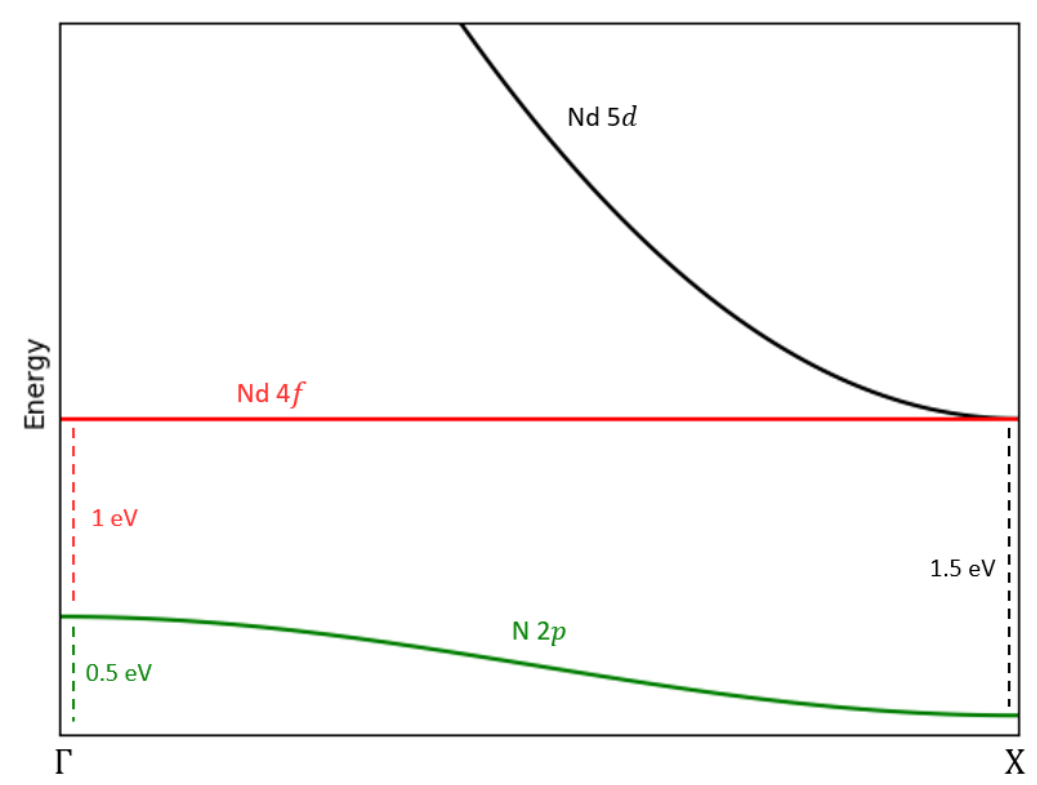

Figure 5.17: Schematic of the band structure of $\mathrm{NdN}$ based on the data shown in Fig. 5.16. The minimum optical gap is $\sim 1 \mathrm{eV}$ between the valence band and $\mathrm{Nd} 4 f$ band at $\Gamma$. Various hybridisation scenarios are possible between the $4 f$ and $5 d$ bands near $\mathrm{X}$, none of which are shown.

caused by a $4 f$ band lying near the conduction band minimum.

A schematic band structure which is consistent with the data in Fig. 5.16 is presented in Figure 5.17. Figure 5.17 shows the $\mathrm{N} 2 p$ valence band and the lowest unoccupied $\mathrm{Nd} 5 d$ and $\mathrm{Nd} 4 f$ bands. Guided by calculations $[2,106,96]$ and experiments on both $\operatorname{GdN}[24,60,61,95]$ and $\mathrm{SmN}[21,63]$ the $5 d$ band is parabolic with a minimum at $\mathrm{X}$. The $4 f$ band is shown in the dispersion-less limit and the $\mathrm{N} 2 p$ band has $\sim 0.5 \mathrm{eV}$ of dispersion between the maximum at $\Gamma$ and the minimum at $X$. The data in Fig. 5.16 indicate that the initial absorption involves transitions from the valence band to the $4 f$ band with a direct gap of $\sim 1 \mathrm{eV}$. In the dispersionless limit, this takes place at $\Gamma$. The $2 p$ to $5 d$ bandgap at $\mathrm{X}$ is difficult to determine from the data available, but extrapolating from the higher energy absorption gives a value of $\sim 1.5 \mathrm{eV}$. The $4 f$ and $5 d$ bands then meet 
at the $\mathrm{X}$ point with a number of hybridisation scenarios possible, none of which are shown.

The only existing calculations of the $\mathrm{NdN}$ band structure offer three possible scenarios [2]. Two of these result in the $4 f$ bands at $\Gamma$ being raised several $\mathrm{eV}$ above the conduction band minimum. This would rule out any optical transitions between these bands in the energy range shown in Fig. 5.16. With only the $5 d$ band remaining, one would expect the form of $\sigma_{1}(\omega)$ for $\mathrm{NdN}$ to then be very similar to that of GdN, with no additional contributions. The third scenario offered is the most consistent with the present data. This calculation shows a minimum optical gap of $\sim 0.6 \mathrm{eV}$ between the valence band and lowest unoccupied majority spin $4 f$ band at $\Gamma$. This $4 f$ band then falls in energy monotonically, hybridising with the $5 d$ band near $\mathrm{X}$, where the direct gap is now some $30 \%$ larger than the gap at $\Gamma$. Although the placement of the bands here seems to reflect the experimental data, this calculation still reaches a semi-metallic conclusion, in contrast to the present results which clearly indicate a non-metallic ground state in un-doped films.

\subsubsection{Temperature Dependant Studies}

Temperature-dependent measurements of both reflection and transmission were conducted on $\mathrm{SmN}$ and GdN films. The absorption was then calculated, along with models for the optical conductivity, at various temperatures above and below the Curie temperature of each material.

\section{Gadolinium Nitride}

Absorption for a GdN film at $250 \mathrm{~K}$ (red), $40 \mathrm{~K}$ (green) and $7 \mathrm{~K}$ (black) can be seen in the top panel of Figure 5.18. Measurements at $250 \mathrm{~K}$, well above the magnetic transition of $\mathrm{GdN}$, are similar to those shown in Figure 5.12 with the absorption increasing strongly above $\sim 1.3 \mathrm{eV}$ due to the direct transition at the $X$ point. Below this the absorption drops to $\sim 2 \%$, which is 


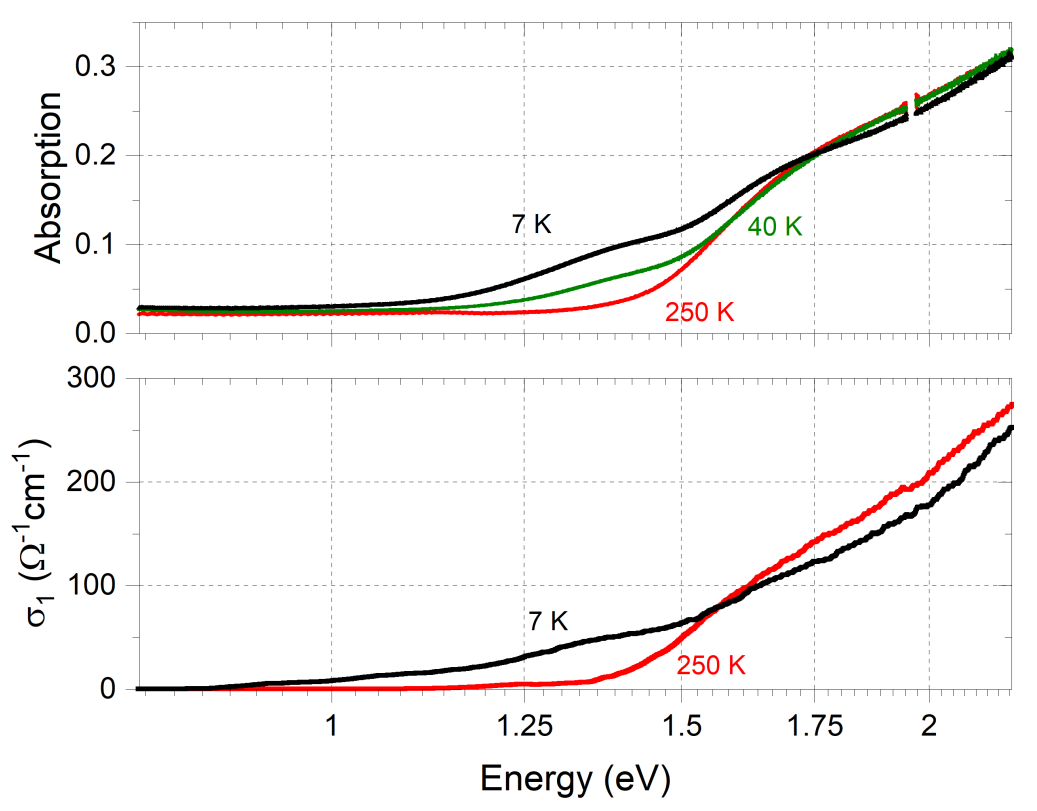

Figure 5.18: Top: Measurements of the absorption of a GdN sample (A556) at various temperatures above and below the Curie temperature. A shift of the optical edge can be seen to lower energy as temperature decreases. Bottom panel: $\sigma_{1}(\omega)$ calculated from the data in the top panel, the same shift to lower energy can be seen in the optical conductivity.

considered essentially zero. Moving to lower temperatures, the $40 \mathrm{~K}$ data show increased absorption with a bandgap closer to $1 \mathrm{eV}$. This increase in absorption at lower energy, or redshift of the optical gap, is caused by spin splitting of the Gd $5 d$ band as the material enters the ferromagnetic phase, reducing (increasing) the bandgap at the $\mathrm{X}$ point for the majority (minority) spin band. Moving now to the lowest temperature measurement at $7 \mathrm{~K}$, absorption can now be seen to be beginning at significantly lower energies leading to a direct bandgap in the ferromagnetic phase of $\sim 0.9 \mathrm{eV}$. The reduction of energy of the optical gap is consistent with previous measurements showing a redshift from $1.3 \mathrm{eV}$ to $0.9 \mathrm{eV}$ [24]. The bottom panel of Figure 5.18 shows $\sigma(\omega)$ calculated from models of the data in the top panel (not shown), only the $250 \mathrm{~K}$ and $7 \mathrm{~K}$ data are shown here for clarity. 
The shift of the optical bandgap to lower energies is now very clear in $\sigma_{1}(\omega)$ when comparing the $250 \mathrm{~K}$ and $7 \mathrm{~K}$ data. The reduction in absorption near $1.8 \mathrm{eV}$ can also be seen here more clearly with the $7 \mathrm{~K}$ data falling below the $250 \mathrm{~K}$ near $1.6 \mathrm{eV}$. Similar to the redshift of the optical gap to lower energies, this decrease in $\sigma_{1}(\omega)$ at higher energies is due to the lifting in energy of the minority spin states when compared to the paramagnetic phase. The majority spin bandgap shifts from $\sim 1.3 \mathrm{eV}$ in the paramagnetic phase to $\sim 0.9 \mathrm{eV}$ in the ferromagnetic phase while the minority spin bands look to shift up to $\sim 1.7 \mathrm{eV}$. This leads to a total spin splitting of roughly $0.4 \mathrm{eV}$ between the paramagnetic and ferromagnetic phases, which is similar to LSDA $+U$ predictions $[2,24]$.

\section{Samarium Nitride}

Temperature-dependent measurements on $\mathrm{SmN}$ yielded only very small changes, with the majority of these occurring between $300 \mathrm{~K}$ and $80 \mathrm{~K}$, some $50 \mathrm{~K}$ above the Curie temperature of $\mathrm{SmN}$. The left-hand panel of Figure 5.19 shows measurements of absorption taken at $250 \mathrm{~K}$ (red) and $5 \mathrm{~K}$ (black) over the region $0.5 \mathrm{eV}$ to $1.9 \mathrm{eV}$ covering both (N $2 p$ to $\mathrm{Sm} 4 f$ and $\mathrm{Sm} 5 d$ ) regions of absorption. Only very small changes in the form of the spectra can be seen between $250 \mathrm{~K}$ and $5 \mathrm{~K}$ in Figure 5.19, thus no shift in the optical edge at $X$ or $\Gamma$ is quoted. The $5 \mathrm{~K}$ spectra can be seen to dip below the $250 \mathrm{~K}$ spectra at high energy, similar to GdN in Figure 5.18, thus this may be due to the increase in energy of the minority spin $5 d$ band at $X$. The reason for the lack of a red-shift of the optical gap at $X$ remains unclear, although hybridisation of the $4 f$ and $5 d$ bands here may reduce the movement of the majority spin $5 d$ band. In any case the tail of the absorption edge is obscured by the low energy feature so only a large shift would be apparent. Moving to the low energy feature, a modest increase can be seen at the peak of the absorption while a decrease near $1.1 \mathrm{eV}$ indicates a slight tightening of the feature.

The changes shown in the left-hand panel of Figure 5.19 are so modest 

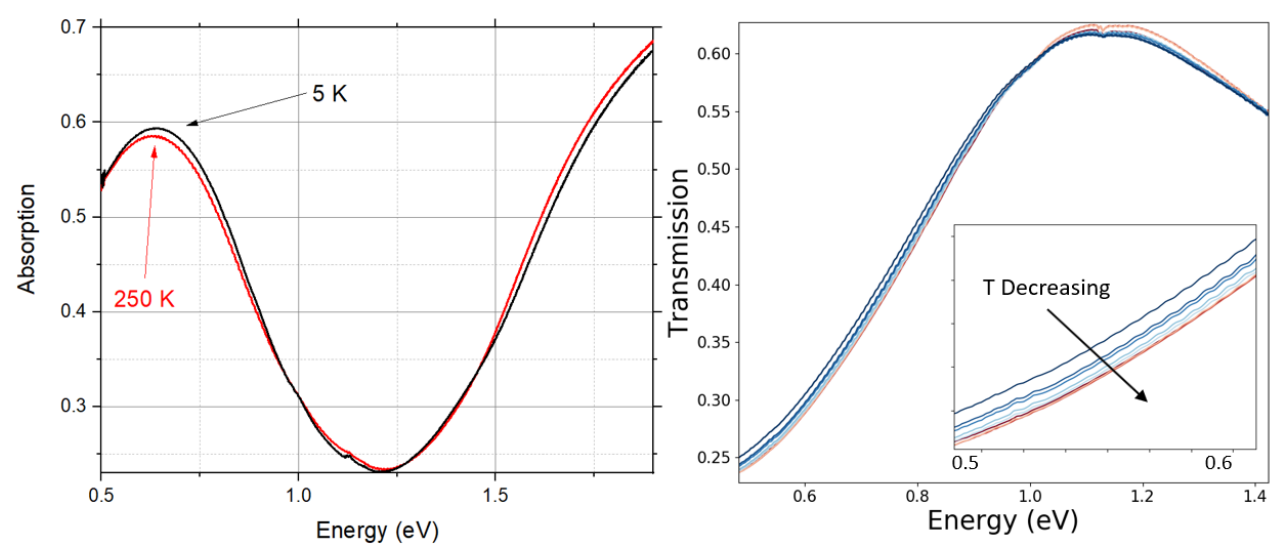

Figure 5.19: Left: The absorption (1-R-T) for a SmN sample at $250 \mathrm{~K}$ (red) and $5 \mathrm{~K}$ (black) based on reflection and transmission measurements taken at each temperature. There is only a slight change in the spectra between the two temperatures. Right: Transmission measurements of the same sample at various temperatures between $250 \mathrm{~K}$ and $5 \mathrm{~K}$. The inset shows that although the change is small over the temperate range, it is continuous.

that it is important to understand that they are reproducible. The righthand panel of Figure 5.19 shows measurements of the transmission at various temperatures between $250 \mathrm{~K}$ and $5 \mathrm{~K}$. The minimum in transmission at low energy corresponds to the peak in absorption in the left-hand plot, similar for the shared features near $1.2 \mathrm{eV}$. Here we can see that at low energy the transmission (absorption) is reducing (increasing) continuously with temperature while at higher energy near $1.2 \mathrm{eV}$ the opposite is true. This is shown more clearly between $0.5 \mathrm{eV}$ and $0.6 \mathrm{eV}$ in the inset. The same behaviour was also seen in a separate measurement of a $\mathrm{SmN}$ film on a Si substrate, however, this is not shown.

\subsection{Conclusions}

Optical measurements over a large energy range were conducted on SmN films, extending the results of previous studies. Low energy measure- 
ments found no evidence of free carriers, supporting the semiconducting ground state commonly found in transport measurements. A phonon absorption was observed near the predicted location, as has been seen in other rare earth nitrides. An absorption feature in the mid / near infrared region has been identified as transitions from the $\mathrm{N} 2 p$ valence band into a low lying Sm $4 f$ band. The location of this band is consistent with transport results reported in Chapter 4.

Measurements on NdN films have also found evidence for a semiconducting ground state, consistent with transport measurements, and observed a phonon mode in a similar region to other rare earth nitrides. An additional contribution to the absorption onset in $\mathrm{NdN}$ has been identified as transitions into a $4 f$ band which meets the $5 d$ band near at the conduction band minimum. The location of this $4 f$ band in $\mathrm{NdN}$ is again consistent with calculations [2], and transport measurements discussed in Chapter 4.

Temperature-dependent measurements on GdN films reproduced previous results regarding the red-shift of the optical gap in the ferromagnetic phase. Low-temperature measurements on $\mathrm{SmN}$ films found only very modest changes in the spectra, which appear significantly above the Curie temperature. 


\section{Chapter 6}

\section{Properties of Doped Rare Earth Nitrides}

This chapter builds on the previous Chapters 4 and 5 with a preliminary analysis and discussion on the electrical transport, magnetic and optical properties specifically in doped rare earth nitrides. To begin, a motivation for studying the doped materials is given along with a brief review of the existing studies of heavily doped rare earth nitrides. Experimental procedures, specifically relating to measurements in doped samples, are then described. The results follow, beginning with structural and optical measurements on doped $\mathrm{NdN}, \mathrm{SmN}$ and GdN films, then electrical transport measurements on $\mathrm{SmN}$ films. The magnetic properties of doped $\mathrm{NdN}$ films and $\mathrm{SmN}$ films are then discussed in the context of previous results on GdN. Finally, a discussion of the observance of superconductivity in appropriately doped $\mathrm{SmN}$ is given.

\subsection{Motivation}

The previous chapters have discussed the interpretation of measurements on largely undoped rare earth nitride films. We now turn to discuss films that are intentionally doped with electrons. 
The importance of understanding the properties of doped rare earth nitrides is high. The majority of the theoretical literature on members of the rare earth nitride series concerns stoichiometric materials, devoid of vacancies. Experimental studies, however, are constrained by the real world, where the inclusion of defects and vacancies of some degree is unavoidable. The low formation energy of nitrogen vacancies $\sim 1 \mathrm{eV}$ [40] means even with current UHV technology, the most carefully prepared films are grown with a vacancy concentration of $\sim 1 \%$ [1]. This problem is compounded by the necessity of high temperatures for epitaxial growths which result in the highest quality materials, in a crystalline sense [14, $15,16,64]$. Growths of films at elevated temperatures can result in a vacancy concentration closer to $\sim 5 \%$, and dramatically reduced resistivites [14, 49].

There are few experimental studies specifically on the transport properties of highly doped rare earth nitride materials [48, 49, 107]. The most complete show $\mathrm{GdN}$, grown at varying ratios of nitrogen to $\mathrm{Gd}$ flux, has a relationship between the vacancy concentration and the Curie temperature, with $T_{c}$ being raised to as much as $200 \mathrm{~K}[48,49]$.

In addition to the clear evidence that the inclusion of nitrogen vacancies has a dramatic effect on the transport properties [10, 11, 108] there is also evidence of lattice distortion [3]. This is a similar scenario to the $\sim 2 \%$ displacement of $\mathrm{Nb}$ ions towards nitrogen vacancy centres in nitrogen deficient $\mathrm{NbN}$ [109]. The combination of changes to the lattice parameter on the order of $\sim 2 \%$, along with the addition of impurity states, will likely have drastic implications on the band structure and exchange mechanism of the rare earth nitrides resulting in changes to both the electronic and magnetic behaviour.

All rare earth nitride materials studied have shown a change of behaviour when doped with electrons, but none more so than $\mathrm{SmN}$, which enters a superconducting phase below $\sim 5 \mathrm{~K}$ when appropriately doped [58]. The origin of superconductivity in $\mathrm{SmN}$ is still unclear. The large spin 
splitting of the $4 f$ bands in $\mathrm{SmN}$ and the positioning of the lowest majority spin $4 f$ band in the conduction band, below the $5 d$ band, at first glance, indicates that the superconductivity likely resides in a spin-polarised heavy Fermion band.

\subsection{Experimental Techniques}

Much of the work discussed in this chapter relies on the same experimental procedures described in Chapter 5 . The specific analysis described here relates to estimating the Curie temperature of $\mathrm{SmN}$ films of varying conductivity, based on temperature-dependent resistivity measurements. The method of characterising the superconducting transition in $\mathrm{SmN}$ films using magneto-resistance measurements is also described.

\subsubsection{Estimating the Curie Temperature in $\mathrm{SmN}$}

The usual method of determining the Curie temperature in ferromagnetic materials is via magnetometry, as described in Section 3.4. The inverse susceptibility is plotted as a function of temperature. In the Curie-Weiss model, the high-temperature signal will have a positive intercept on the $\mathrm{x}$ axis, which is defined as the paramagnetic Curie temperature. Although this is still true for $\mathrm{SmN}$, the experimental situation is complicated by the small net moment of the $\mathrm{Sm}^{3+}$ ion, which has been discussed previously. The small moment, which couples very weakly to an external field, along with contributions of a similar order from substrates and capping layers, and the temperature-independent Van Vleck contribution, make magnetometry largely impractical on thin films of $\mathrm{SmN}$ as a routine measurement. Here we use an experimentally simpler method, which will not strictly determine the Curie temperature but rather a characteristic temperature relating to the ferromagnetic transition, which here we use as a proxy. This temperature is determined via measurements of the resistivity 

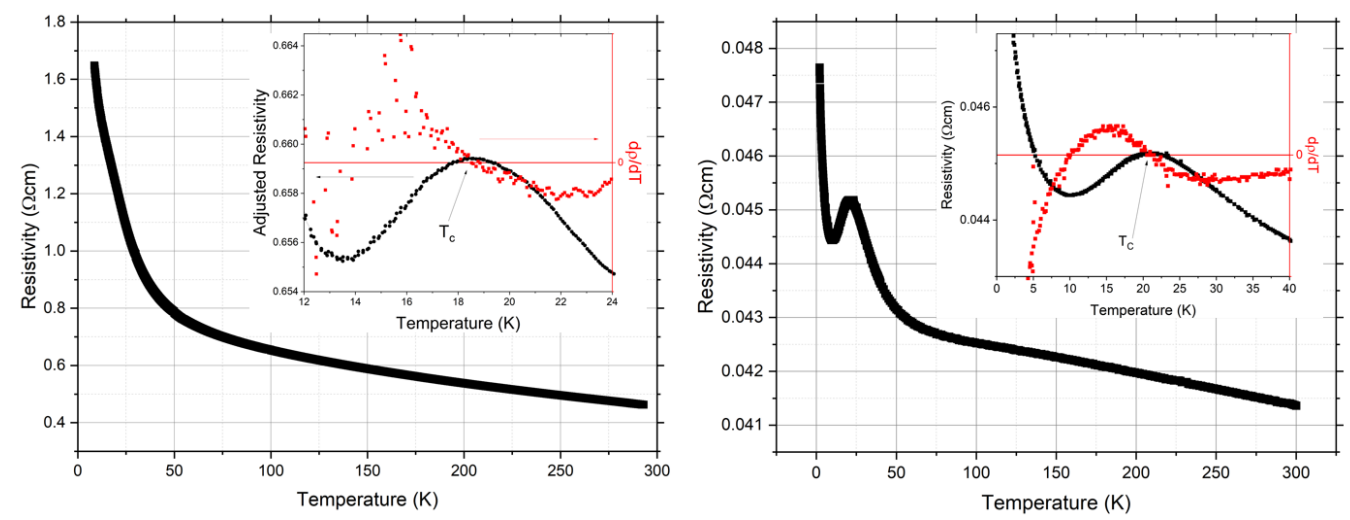

Figure 6.1: Left: The magnetic transition in the resistive sample (A547) is mostly obscured by the diverging resistivity at low temperatures, this is removed in the inset and the zero crossing of the derivative used to estimate the Curie temperature. Right: A conductive sample (A584) does not require the removal of the low-temperature rise to estimate the Curie temperature.

as a function of temperature, which is now described.

In ferromagnetic transition metals a peak in the temperature derivative of resistivity is expected to be related to the ferromagnetic transition $[110,111]$. The picture is changed in the semiconducting rare earth nitrides, where the ferromagnetic transition is now signalled by a peak in the resistivity rather than the temperature derivative [107, 112]. The most reliable method to determine the location of the peak in the resistivity is to observe $d \rho / d T$ and find the zero crossing, then use this as a proxy of the Curie temperature. This is easily done in relatively conductive samples where the resistivity shows a clear peak, but complicated in more resistive samples where the low-temperature data is dominated by the diverging resistivity. In this case the low-temperature resistivity is, to first order, assumed to be described by as $\rho(T) \propto \exp \left(U / k_{B} T\right)$ where $U$ is some activation energy and $k_{B}$ is the Boltzmann constant. The diverging component can be removed by fitting the low energy data to an exponential term, removing the result of the fit, then taking the temperature derivative and finding the zero crossing from the remaining data. An example of this 
process is shown in Figure 6.1 for both a conductive and non-conductive film. The left-hand panel of Figure 6.1 shows the resistive film. At low temperatures the resistivity increases quickly with a slight indication of an inflection near $20 \mathrm{~K}$. The inset shows the data between $10 \mathrm{~K}$ and $24 \mathrm{~K}$ once an exponential increase has been removed (black), where a peak is now visible in the data. The temperature derivative is also shown in the inset (red) which has a zero crossing, signalling the characteristic temperature related to the ferromagnetic transition, at $18 \mathrm{~K}$. The right-hand panel of Figure 6.1 shows the same process on a more conductive sample. The peak is clearly visible in the main figure. The inset shows the same data below $50 \mathrm{~K}$ (black) and the temperature derivative (red) with a zero-crossing at $21 \mathrm{~K}$.

\subsubsection{Characterising the Superconducting State of $\mathrm{SmN}$}

The critical temperature in $\mathrm{SmN}$ samples has previously been observed to be as high as $3 \mathrm{~K}$ [58], however, most samples appear to have a critical temperature below $2 \mathrm{~K}$. This complicates experiments as the lowest temperature readily available in our measurement systems is $\sim 2 \mathrm{~K}$. Due to this, the onset temperature $T_{\text {on }}$ will be referred to rather than the critical temperature. The onset temperature is estimated by noting the highest temperature that behaviour which can be explained by any portion of the sample entering a superconducting state is observed, via any measurement (i.e., temperature-dependent resistance measurements or magnetoresistance measurements).

The data suggest that in the majority of samples the superconductivity is inhomogeneous, resulting in broad and incomplete transitions, which are difficult to characterise. The coexistence of this inhomogeneous superconductivity and the non-metallic temperature-dependent resistivity of $\mathrm{SmN}$ makes the superconductivity even more difficult to observe. In a simple temperature-dependent measurement there is now competition 


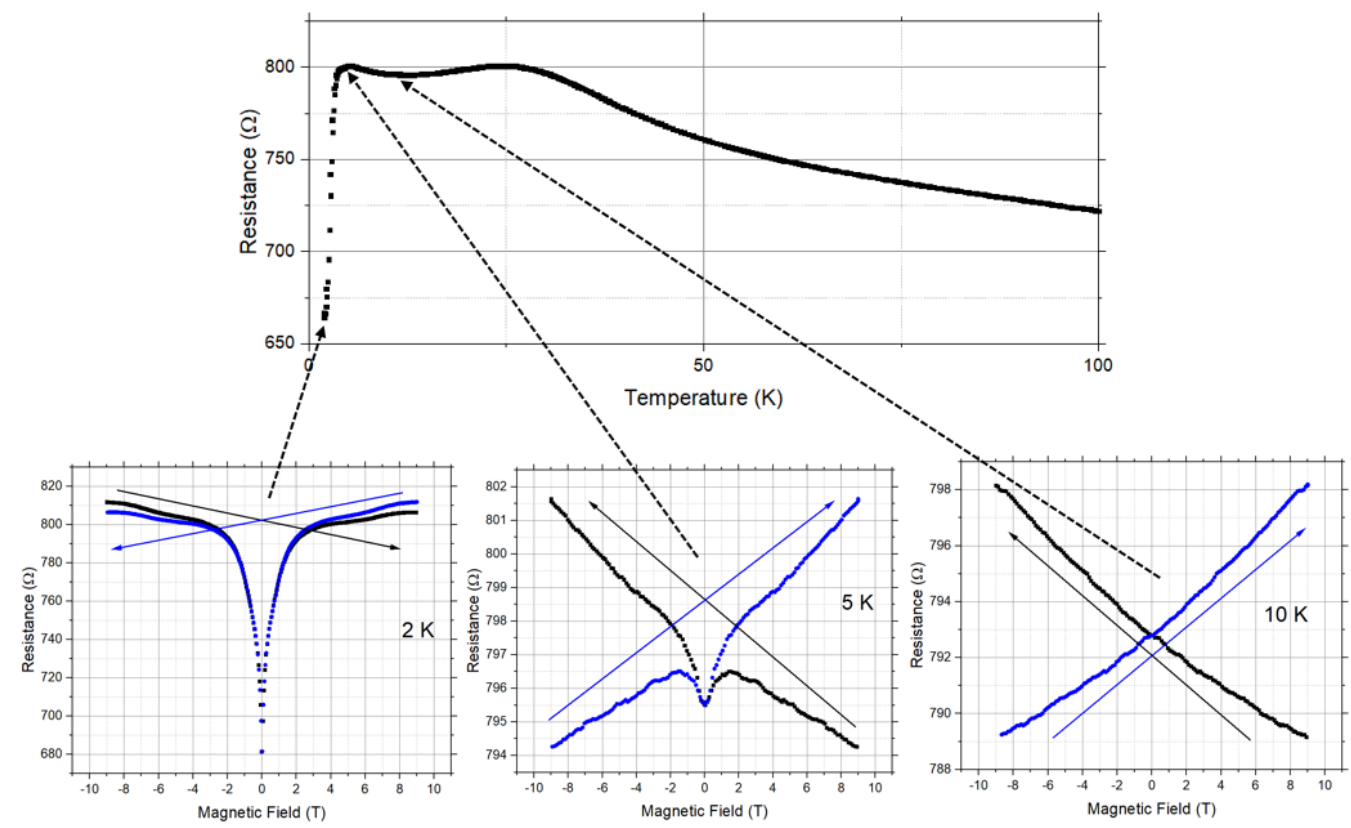

Figure 6.2: Top: Plot of the resistance as a function of temperature for a $\mathrm{SmN}$ sample (A480 A). The resistance initially increases as temperature decreases showing the non-metallic nature of the sample. There is a peak in the resistance near $27 \mathrm{~K}$ related to the ferromagnetic transition. At low temperatures the resistance decreases sharply as the sample enters a superconducting state. Bottom: Resistance as a function of applied field at various temperatures showing the onset of superconductivity, at higher temperatures than it is apparent in the top panel.

between these two states (superconducting and non-metallic). Due to this competition, it is sensible to determine the onset temperature not via temperature-dependent resistance measurements, but by measuring the resistance at a stable temperature while sweeping a magnetic field. Here if portions of the sample are in a superconducting state, there appears a positive magneto-resistance around zero field as the superconducting state is destroyed by the applied field. This contrasts the usual negative magnetoresistance of $\mathrm{SmN}$ observed in the absence of any superconductivity.

Figure 6.2 shows measurements of the resistance as a function of temperature and magnetic field for a $\mathrm{SmN}$ sample. The top panel shows the 
resistance initially increases as temperature is reduced. There is then a peak near $27 \mathrm{~K}$ related to the magnetic transition after which the resistance continues to increase until $\sim 4 \mathrm{~K}$. Between $4 \mathrm{~K}$ and $2 \mathrm{~K}$ the beginning of a superconducting transition can be seen with the resistance quickly falling. The lower panel of Figure 6.2 shows the resistance as a function of magnetic field at three temperatures. The $10 \mathrm{~K}$ measurement shows simple behaviour, along with a large hysteresis expected in the ferromagnetic phase of $\mathrm{SmN}$ (note the magneto-resistance is expected to be symmetric about the magnetisation $M=0$, as discussed in Section 4.3). The $5 \mathrm{~K}$ measurement now shows an additional feature, the appearance of positive magneto-resistance around zero field. This is interpreted as portions of the sample entering a superconducting state, then upon application of a magnetic field larger than the critical field, being driven back into a nonsuperconducting state. Finally, the $2 \mathrm{~K}$ measurement shows a much more dramatic contrast between the zero field and high field states. It is clear from this data that portions of the sample enter a superconducting phase near $5 \mathrm{~K}$ while the total sample resistance is still increasing. For this sample, the onset temperature would be determined as $5 \mathrm{~K}$ as this is the highest temperature any sign of superconductivity was observed.

\subsection{Results}

\subsubsection{Structural Measurements}

Films of GdN, SmN and NdN were grown as previously described, but now with an elevated substrate temperature, and (or) a reduced nitrogen pressure, to promote the formation of nitrogen vacancies. Figure 6.3 shows XRD $2 \theta$ scans of the doped and un-doped samples as well as the measured lattice parameters. The undoped samples (black) in the left-hand panel of Figure 6.3 show the usual (111) texture of polycrystalline rare earth nitride films. Continuing to look at the 111 peak, but now in the doped 

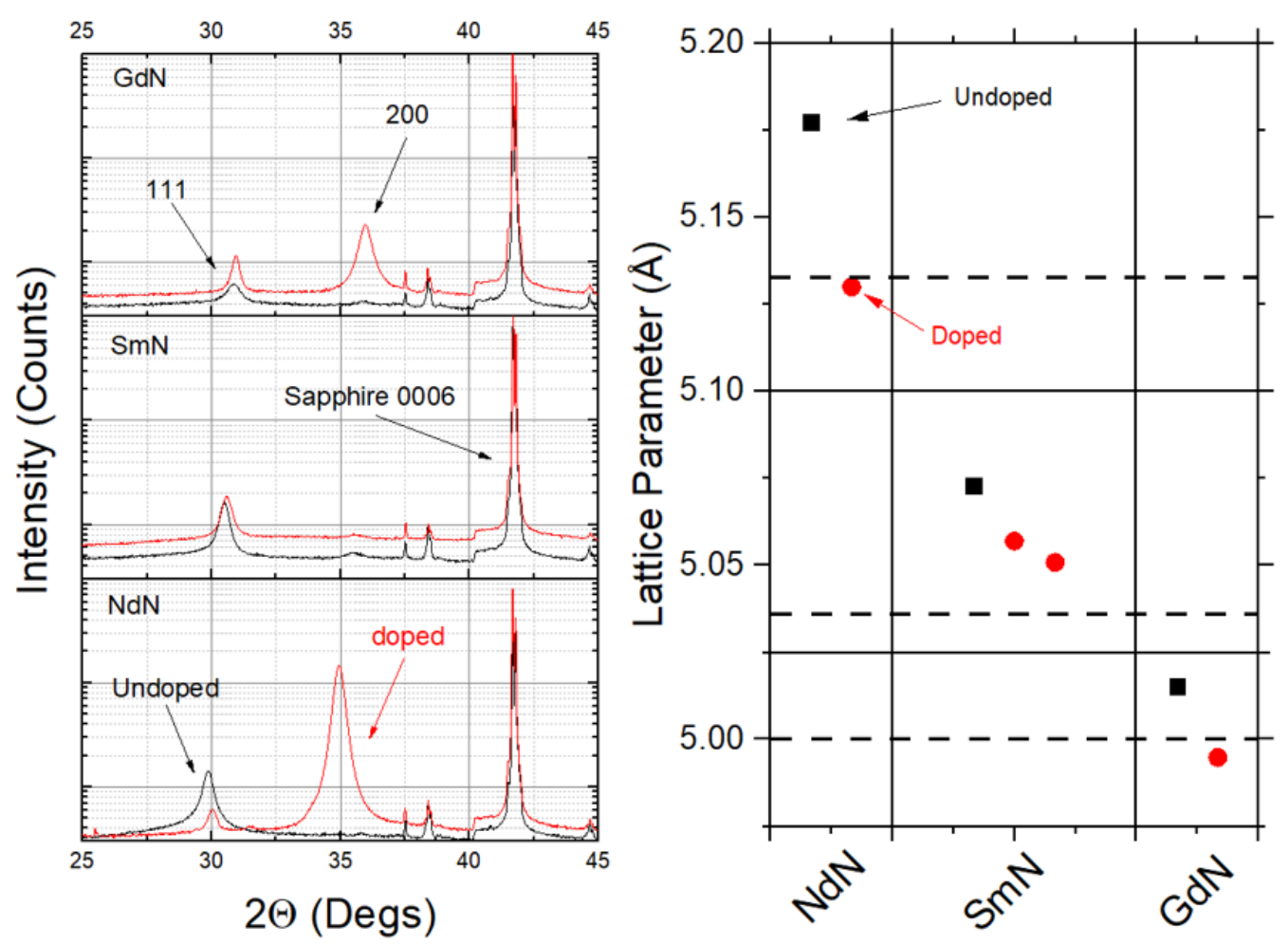

Figure 6.3: Left: XRD $2 \theta$ plots of doped (red) and undoped (black) GdN, SmN and NdN films. Right: Lattice parameters determined from the left hand plot. The three dashed horizontal lines depict the literature values for $\mathrm{NdN}, \mathrm{SmN}$ and GdN respectively [1], read top to bottom.

films (red), this can be seen systematically shifted to slightly higher angles over all three materials. GdN and NdN also show a significant change in the weighting from the (111) to (100) orientation with the latter becoming dominant in these two materials. The right-hand panel of Figure 6.3 shows the lattice parameters determined from the data in the left-hand panel. In all cases the doped samples now clearly show a reduced lattice parameter on the order of $0.5 \%$. The horizontal dashed lines on the right-hand panel of the figure show the literature (historical) lattice parameters for each material [1]. It is clear that the doped samples have a lattice parameter closer to these literature values. It seems likely that samples used in early studies 
of the rare earth nitrides suffered from unintentional nitrogen vacancies leading to the reduced lattice parameters. These unintentional nitrogen vacancies, which are expected to dope the material with electrons, may explain the confusion regarding the ground state of many of the rare earth nitrides (i.e. semi-conducting or semi-metallic) in the early literature.

\subsubsection{Optical Measurements}

Measurements of reflection and transmission for doped GdN and $\mathrm{NdN}$ samples were taken as described in section 5.2. However, for these doped materials, only sapphire substrates were used. Si substrates were not used as a rare earth silicide is formed at a $\mathrm{Si} / \mathrm{L}$ interface when the rare earth is deposited onto $\mathrm{Si}$ at high temperature [113]. Results for the reflection, transmission and resulting absorption are shown in Figure 6.4 along with the model for the absorption alone. Similar to the insulating samples, both GdN and NdN show a decrease in transmission above $\sim 1 \mathrm{eV}$ and $\sim 0.7 \mathrm{eV}$ respectively, which is caused by the onset of interband transitions. There is now, however, an additional decrease in the transmission measurements at lower energy, seen in the $\mathrm{GdN}$ data as a reduced transmission between $0.2 \mathrm{eV}$ and $0.7 \mathrm{eV}$, and similarly in the NdN measurement. This reduced transmission should not be confused with the sharp phonon absorption in the sapphire substrate where the transmission quickly falls to zero below $\sim 0.2 \mathrm{eV}$. The absorption in the sapphire substrate is shown in the lower right-hand panel of Figure 6.4 for reference.

The lower panels of Figure 6.4 show the absorption (1-R-T) for the doped GdN and NdN samples. As in the insulating samples, a strong increase in the absorption can be seen above $\sim 1 \mathrm{eV}$. An additional absorption at lower energy is now more apparent as an increasing absorption with decreasing energy below $\sim 1 \mathrm{eV}$ in the $\mathrm{GdN}$ and $\sim 0.7 \mathrm{eV}$ in the NdN sample, which signals a free carrier absorption. The reflection and transmission data were modelled as previously described and combined 

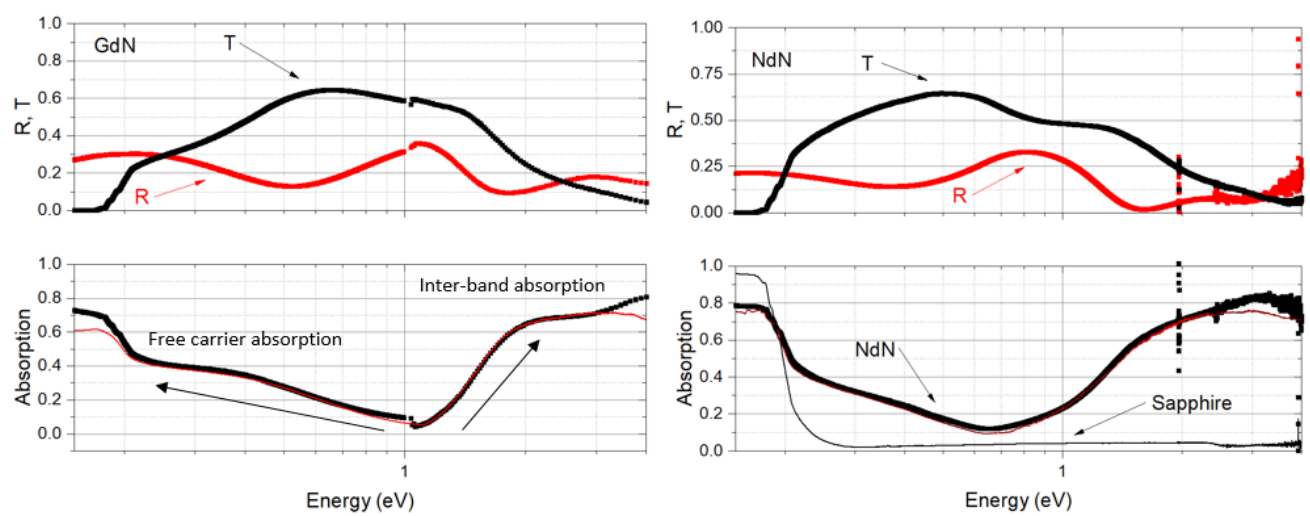

Figure 6.4: Plots of the measured reflection, transmission and absorption (1-R-T) for doped GdN (A612) (left) and NdN (A613) (right) on sapphire substrates. The onset of interband transitions can be seen in each material above $\sim 1 \mathrm{eV}$ similar to the insulating samples. At lower energy (below $\sim 0.7 \mathrm{eV}$ ) there is now an increased absorption, caused by free carriers in each material. The bottom panel show a fit to the data (red).

to give a model of the absorption, shown in red in the lower plots. In these models a single Drude-like free carrier term was used at zero energy to describe the features below $\sim 0.7 \mathrm{eV}$. The models each represent the data well, some deviation can be seen at high energy where there is little light transmitted through the samples, and at low energy where there are strong contributions from the phonon in sapphire.

As opposed to the GdN and NdN samples grown at elevated temperature, SmN samples were grown on both sapphire and Si substrates. Prior to growth of the SmN an AlN buffer layer was deposited onto the Si substrate to protect against the rare earth silicide formation [113]. Plots of the reflection, transmission and absorption as a function of energy for the most conductive SmN sample can be seen in Figure 6.5. The top panel of Figure 6.5 shows the reflection and transmission, where the effect of the free carriers can be clearly seen with the enhanced reflectivity at low energy. In the lower plot the absorption can be seen, along with a model 

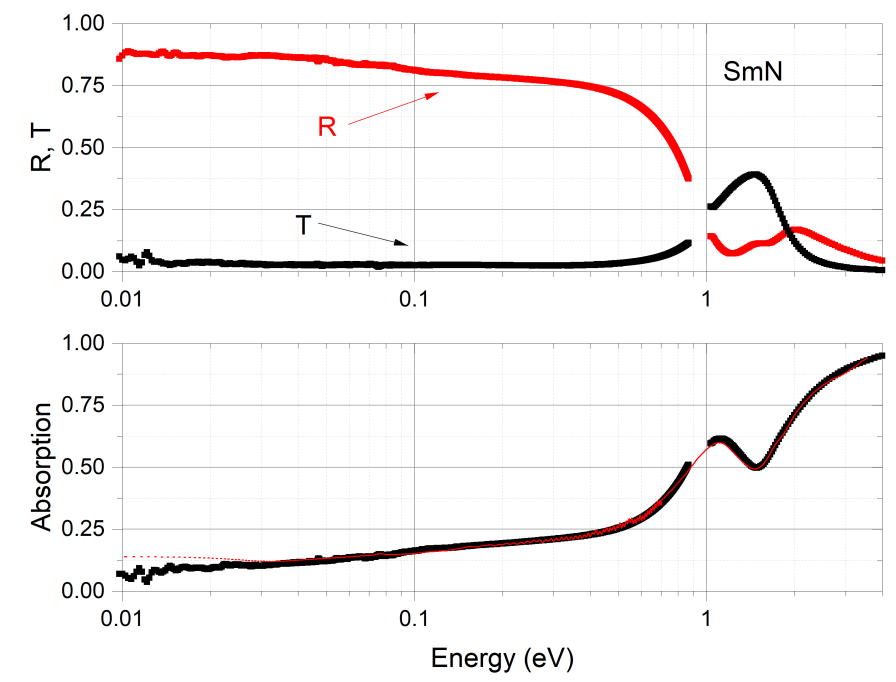

Figure 6.5: Top: Measurements of reflection and transmission on a conductive SmN sample (A610). Low energy measurements are taken from a sample grown on AlN buffered Si, high energy measurements are taken on a sapphire substrate. Bottom: Absorption (1-R-T) determined from the data in the top panel along with a model (red) of the data.

based on fitting the reflection and transmission data above.

\section{Optical Conductivities}

The models shown above were used to determine the optical conductivity of the doped GdN, NdN and SmN samples; these are now compared with the insulating samples discussed in the previous chapter. Figure 6.6 shows the optical conductivity for doped (red) and undoped (black) samples of GdN (left) and NdN (right). Beginning at low energy, a significant contribution can be seen resulting from the free carrier term which we have written, using the Drude form, as

$$
\sigma_{1}(\omega)=\frac{\omega_{p}^{2} \tau}{1+\omega^{2} \tau^{2}}, \quad \omega_{p}^{2}=\frac{n e^{2}}{m \epsilon_{0}} .
$$

By fitting the low energy data the carrier concentration $n$ and scattering 

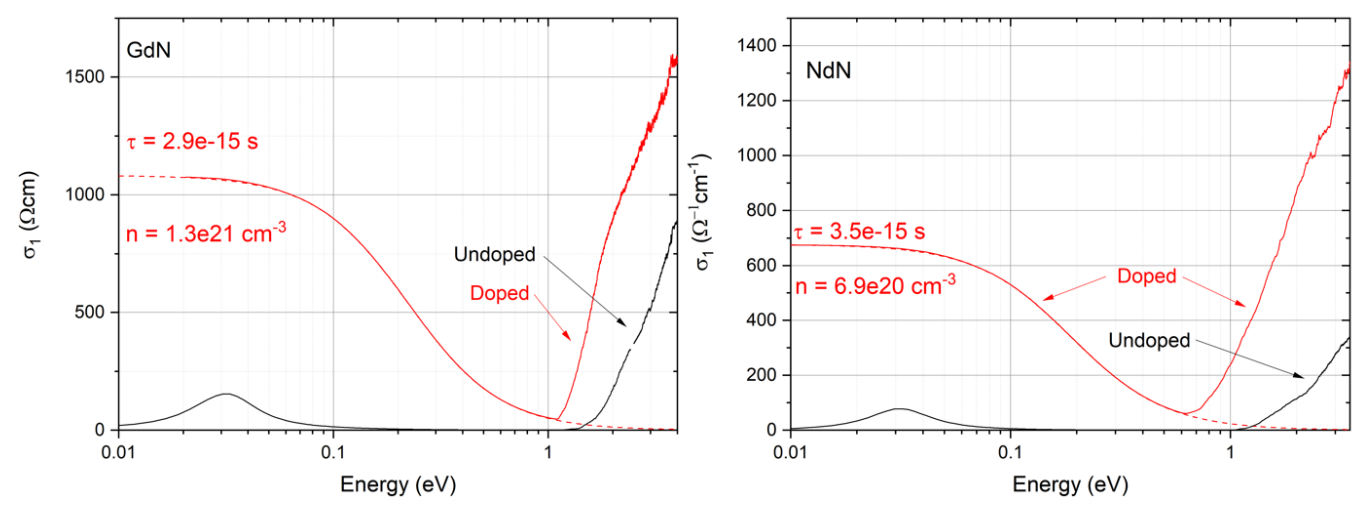

Figure 6.6: Plots of the real part of the optical conductivity of the doped GdN and $\mathrm{NdN}$ samples (red) along with the insulating samples (black). The conductive samples have a large low energy contribution from the free carriers in the material. The carrier concentration and scattering times extracted from the low energy fits are printed on the figure.

time $\tau$ can be determined, with some assumed effective mass. The carrier concentrations and scattering times for each sample can be seen on the plot, calculated with $m^{*}=1$. Using these values for $n$ and $\tau$ an estimate of the mean free path of an electron in the material can be made assuming a thermal velocity, which results in $\sim 3.2 \AA$ and $\sim 3.8 \AA$ for the GdN and NdN samples respectively. The mean free path determined via this method is the same order as the measured lattice parameter in each sample so provides at least a possible value. For the GdN sample the same calculation can be made using the Fermi velocity resulting in a mean free path of $\sim 1.5 \mathrm{~nm}$, roughly a factor of five larger than the previous value. The $4 f$ level threading through the conduction band minimum in $\mathrm{NdN}$ makes estimating the Fermi energy and analysis using the Fermi velocity less straight forward.

Moving to the higher energy region interband absorption can be seen to begin near $1 \mathrm{eV}$. The tail of the zero frequency term can be seen to extend into this region as the dashed red line in each plot. Even with this additional contribution at the foot of the interband absorption it is clear that 

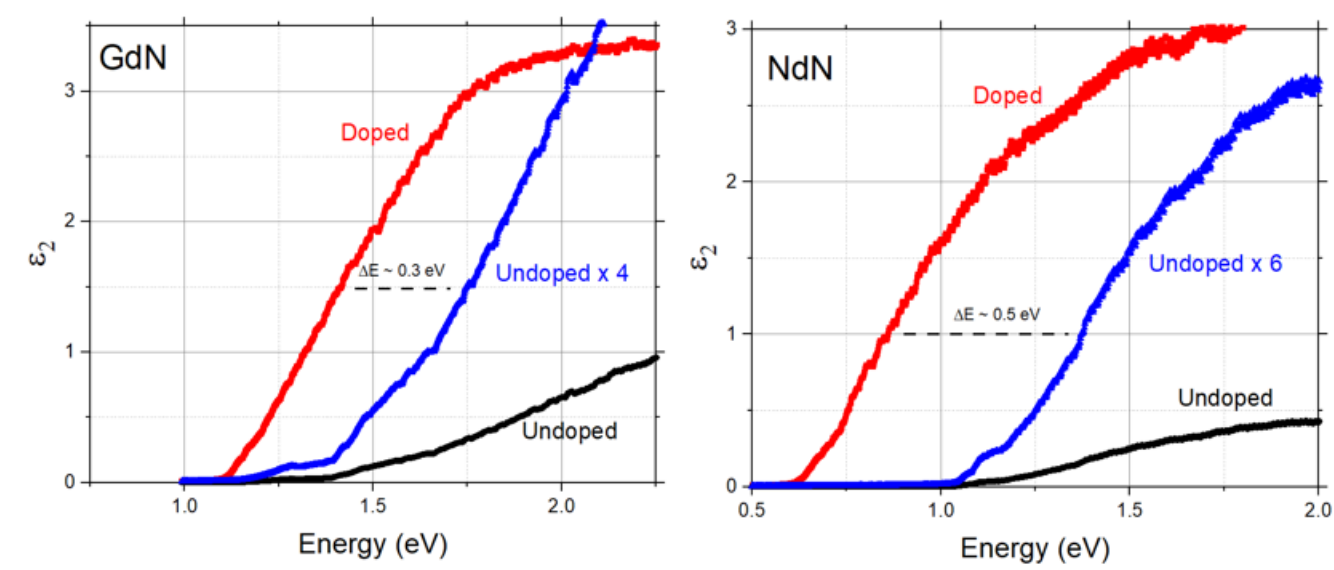

Figure 6.7: Plots of the imaginary part of the dielectric function $\epsilon_{2}$ for conductive and insulating $\mathrm{GdN}$ and $\mathrm{NdN}$. The form of $\epsilon_{2}$ for the conductive samples can be seen to be roughly a multiplicative factor stronger than the insulating plus a transposition to lower energy. This transposition is used to determine the difference of bandgap between respective conducting and insulating samples.

the conductive samples have a red-shifted optical gap, and an enhanced magnitude when compared to the insulating samples.

To compare the insulating to conductive samples the shift between them has been measured. The optical conductivity scales with energy thus accentuates the higher energy features somewhat, so for this analysis we turn to the imaginary part of the dielectric function. $\epsilon_{2}$ is shown in Figure 6.7 in the interband region (note that the free carrier contributions have been removed). Here we see that to a good approximation $\epsilon_{2}$ for the conductive samples has the same form as the insulating samples, though stronger by some factor. When $\epsilon_{2}$ for the insulating sample is scaled a transposition can then be seen. This transposition was then used to determine the change in bandgap.

Moving on now to $\mathrm{SmN}$, Figure 6.8 shows both the DC resistivity as a function of temperature and the optical conductivity at room temperature for three samples of varying conductivity, the most conductive being the sample from Figure 6.5. The bottom sample (blue) in the left-hand panel 

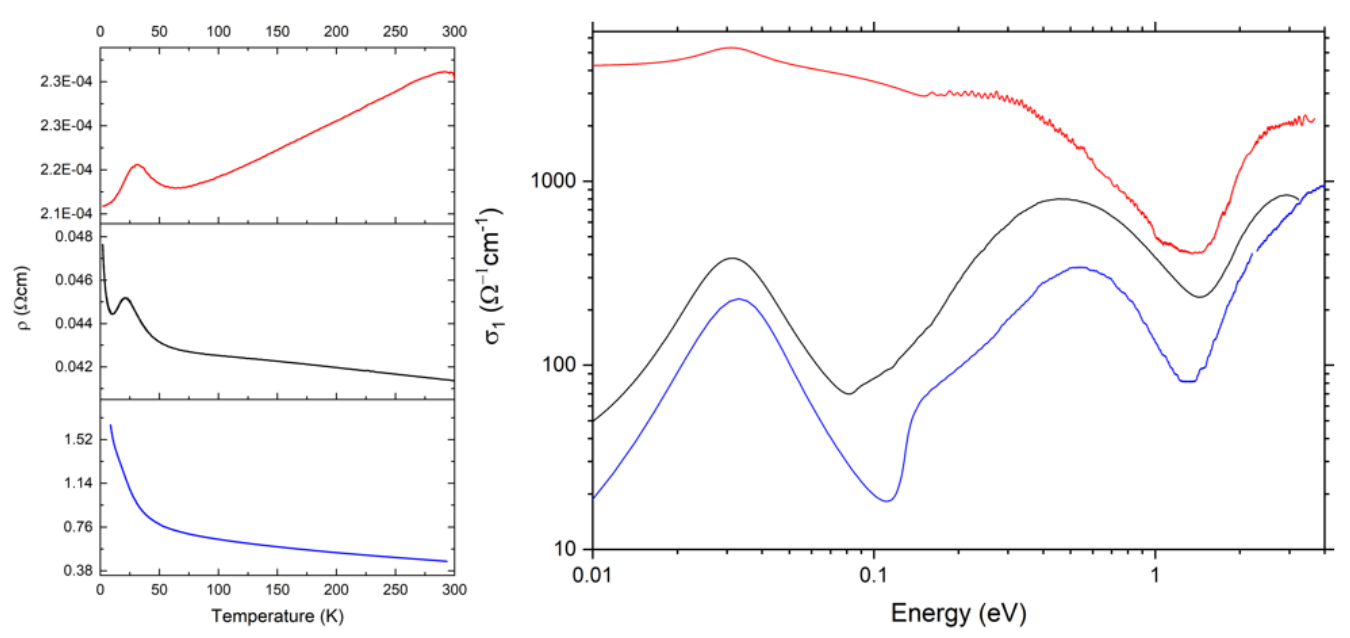

Figure 6.8: Left: Plots of resistivity as a function of temperature for three $\mathrm{SmN}$ films of varying doping. Right: Optical conductivity at room temperature for the same films shown in the left hand panel.

is the most resistive and shows non-metallic behaviour below room temperature. This sample shows the largest increase in resistivity at low temperatures over all three samples. There is an inflection near $18 \mathrm{~K}$, related to the magnetic transition, which is mostly obscured by the diverging resistivity at low temperature. Over the temperature range of $\sim 300 \mathrm{~K}$ the resistivity increases by a factor of $\sim 4$. The centre plot shows a moderately conductive sample. Here again we see non-metallic behaviour, the resistivity increasing as temperature decreases. The increase in resistivity in this sample is a more modest $\sim 10 \%$ over the same temperature range. The peak relating to the magnetic transition is more clearly visible and at a slightly higher temperature than the previous $\sim 21 \mathrm{~K}$. The top plot in the left-hand panel of Figure 6.8 shows the most heavily doped, and thus conductive sample. Here we see a positive temperature coefficient of resistance which contrasts the previous two plots. This sample also shows a small change in resistivity of $\sim 10 \%$. The peak in the resistivity near the Curie temperature is again easily visible and at a higher temperature still $\sim 30 \mathrm{~K}$. It is clear that this sample is doped to degeneracy. 


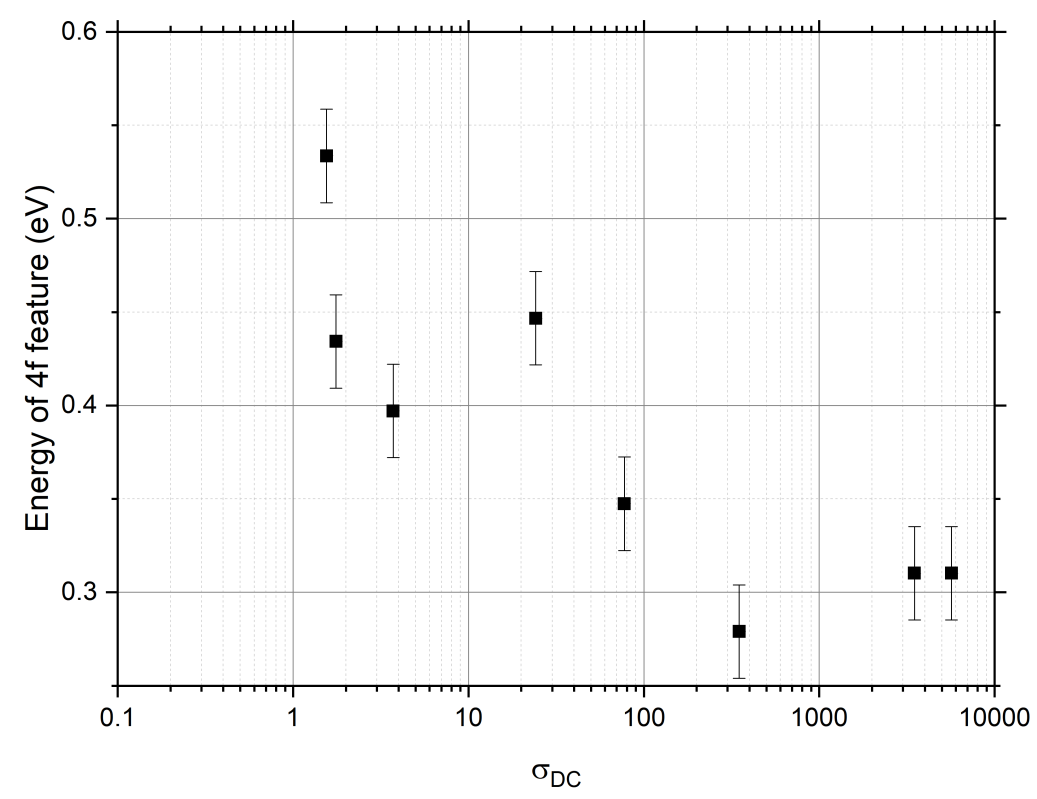

Figure 6.9: Plot of the centre of the feature relating to transitions between the $\mathrm{N} 2 p$ and $\mathrm{Sm} 4 f$ bands as a function of conductivity for a series of SmN samples.

The right-hand panel of Figure 6.8 shows the optical conductivity from the same three $\mathrm{SmN}$ samples. Here there is a similar trend to the GdN and $\mathrm{NdN}$ measurements previously discussed, although the edge of the optical absorption near $1 \mathrm{eV}$ is now largely obscured by the lower energy absorption feature. Instead we focus on the lower energy feature discussed in Chapter 5 which is caused by transitions from the $\mathrm{N} 2 p$ valence band into a flat $\mathrm{Sm} 4 f$ band. Similar to the $\mathrm{GdN}$ and NdN samples, the magnitude of this feature looks to be increasing in the more conductive $\mathrm{SmN}$ samples, along with a shift to lower energy. To determine the red-shift more quantitatively the low energy (free carrier) term was first removed from the data before the centre of this feature was determined. This analysis was completed for the entire series of $\mathrm{SmN}$ samples measured and can be seen in Figure 6.9, plotted as a function of DC conductivity ( $\sigma_{D C}$ and optical measurements are both at $300 \mathrm{~K}$ ). The data in Figure 6.9 show a clear trend with the peak moving to lower energy as conductivity increases. 


\section{Observed redshift of the absorption edge in conductive samples}

The results of the shifting of features in the optical spectrum of all three materials are now considered. We discuss five distinct contributions commonly associated with the optical bandgap in doped semiconductors:

\section{Burstein Moss effect}

Blue shift in optical absorption edge due to carriers doped into the bottom of the conduction band [114].

2. Reduction of lattice parameter

Decrease in the bandgap due to an increased dispersion and widening conduction and valence bands at $X[33,50]$.

3. Electron-electron interaction, screening by free carriers

Decrease in the bandgap due to screening effects from dopant electrons $[115,116]$.

4. Addition of impurity states some meV below the conduction band minimum

Impurity states can now be the initial or final states of new optical transitions not present in the band structure of un-doped samples [40].

5. Effects of disorder caused by nitrogen vacancies

Partial loss of $k$ dependence in optical transitions causing an apparent decrease in the optical gap and an increase in absorption at a given energy [117, 118].

For clarity, a fuller discussion of the above mechanisms will be given in the context of the band structure of $\mathrm{GdN}$, along with estimates of the magnitude of the effect on the optical bandgap. 


\section{Burstein Moss effect}

If $\mathrm{GdN}$ is considered in a simplistic picture, not considering the creation of impurity states near the conduction and valence band extrema, then as electrons are doped into GdN one expects that they will occupy states at one of the three equivalent $\mathrm{X}$ points in the roughly parabolic Gd $5 d$ band. This will result in a blue shift of the Fermi level as now there are filled states in the conduction band [114]. The lowest energy optical transition is now the intrinsic bandgap $E_{g}$ plus the additional energy required to overcome the occupied states at the bottom of the conduction band $\Delta_{B M}$. An estimate of this additional energy $\Delta_{B M}$ can be determined by simply integrating the density of states

$$
n(E)=\int D(E) d E=\frac{1}{2 \pi^{2}} \int_{0}^{\epsilon} d E\left(\frac{2 m_{e}}{\hbar^{2}}\right)^{3 / 2} \sqrt{E-E_{0}} .
$$

To achieve the measured carrier concentration in the GdN sample $\left(\sim 1 \times 10^{21} \mathrm{~cm}^{-3}\right)$ the upper limit of the integration, or the approximate Burstein Moss shift $\Delta_{B M}$ is $\sim 0.8 \mathrm{eV}$, found using a density of states effective mass of $m^{*} / m_{e}=$ 1 and a simple parabolic dispersion. The data in Figure 6.7 shows a decrease in the optical gap of $\sim 0.3 \mathrm{eV}$ rather than the $0.8 \mathrm{eV}$ increase expected from a simplistic Burstein Moss effect. A significant reduction in the bandgap of $\sim 1.1 \mathrm{eV}$ is now required to account for the redshift in the measurement and the Burstein Moss shift. It should be noted that this is indeed a very significant reduction. In the paramagnetic phase the calculated absolute bandgap in $\mathrm{GdN}$ is $\sim 0.8 \mathrm{eV}$ thus the reduction required here clearly places the heavily doped $\mathrm{GdN}$ in a semi-metallic ground state, in the picture of a simple static shift in the band structure.

\section{Reduction of lattice parameter}

Experimentally and in theory the varying of lattice parameter with growth conditions has been seen in rare earth nitrides by various groups [3, 4, $1,40]$, with two groups relating this directly to nitrogen vacancies in the 
lattice. This was thought to be a scenario similar to what has been observed in nitrogen deficient $\mathrm{NbN}$, where the presence of nitrogen vacancies reduces the spacing between the surrounding $\mathrm{Nb}$ ions to the order of $2 \%$ [109].

The electronic structure of a material is sensitive to lattice parameter. In the context of the rare earth nitrides an expanded (contracted) lattice will tend to decrease (increase) dispersion in the $5 d$ band; this leads to an increased (reduced) bandgap at the X point [33]. With this in mind, it is reasonable that the reduced lattice parameter of the doped films can, in part, account for the reduction of the bandgap. To estimate the size of the effect in GdN the bandgap was determined at various lattice parameters using a DFT framework in the LSDA $+U$ approximation.

We have used the Quantum Espresso code [119] and pseudopotentials from Topsakal et. al [23] to calculate the band structure of $\mathrm{GdN}$ at various lattice parameters which were then used to determine the optical bandgap at X. A $U$ parameter is generally applied to electrons in the $f$ and $d$ shells simultaneously and referred to as $U_{f}$ and $U_{d}$ respectively. The $U_{f}$ term is based on the on-site repulsion experienced by the largely localised $4 f$ electrons and can be determined from experiment $[2,33]$. The $U_{d}$ term accounts for the over screening of long-range coulomb effects by the free electron gas used in LDA calculations [24, 33] and is largely empirical. Due to limitations of the Quantum Espresso code, only a single $U$ parameter could be applied; thus $U_{f}$ alone was used. Although the lack of a $U_{d}$ parameter means it is not possible to directly compare the calculated and experimental results it is still possible to observe the rate of change of the bandgap with respect to the lattice parameter in the calculated results. This can then be used with the measured change in lattice parameter between the doped and undoped samples to determine an estimate of the effect of that lattice parameter on the optical gap in the experimental data.

Figure 6.10 shows a selection of the calculated band structures of GdN at various lattice parameters. These results are largely consistent with a 

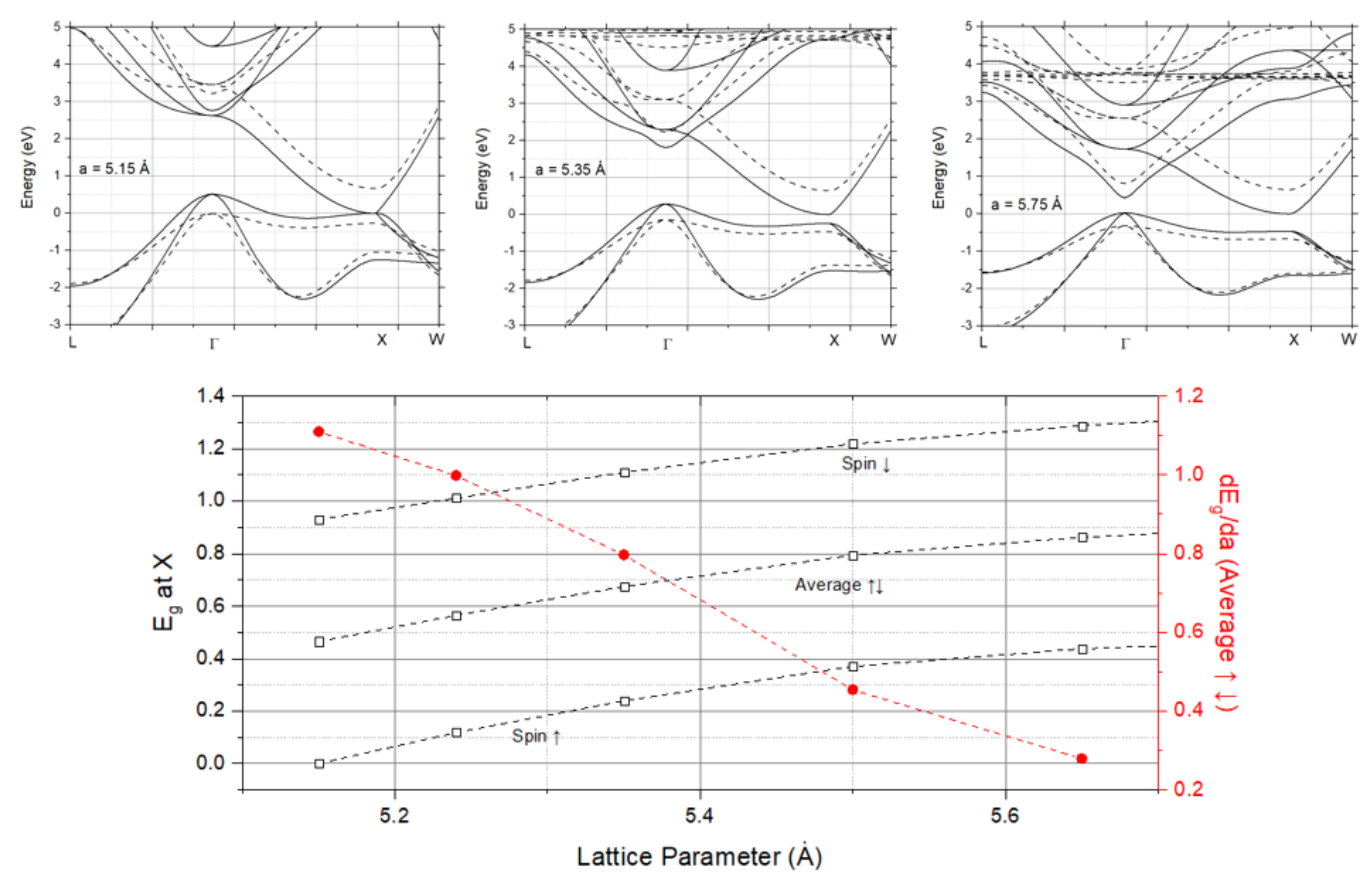

Figure 6.10: Top: plots of the band structure of $\mathrm{GdN}$ at three different lattice parameters. Bottom: Majority, minority and average bandgaps at $\mathrm{X}$ as a function of lattice parameter (black), the derivative of the average gap (red).

similar study which also investigated the band structure of $\mathrm{GdN}$ as a function of lattice parameter using LSDA $+U_{f}$ [50]. The present calculations were repeated using two $U_{f}$ parameters $\left(U_{f}=6.7 \mathrm{eV} \mathrm{[50]} \mathrm{and} U_{f}=9.2 \mathrm{eV}\right.$ [2]) for comparison. The results were largely independent of the selection of $U_{f}$, which is not so surprising given the large separation between the flat $4 f$ bands and $5 d$ conduction band minimum in $\mathrm{GdN}$, which is achieved with either $U_{f}$ (results presented are using $U_{f}=9.2 \mathrm{eV}$ ).

Figure 6.10 shows that as the lattice parameter is increased from $5.15 \AA$ to $5.75 \AA$ the bandgap at $X$ opens while the gap at $\Gamma$ reduces. The bottom panel of Figure 6.10 shows the calculated bandgap at $X$ as a function of lattice parameter for the spin up bands, spin down bands and an average of the two, the last of these loosely represents the paramagnetic phase [24]. The derivative of the average gap is also shown in red. Results in the lower 
panel of Figure 6.10 show that as the lattice begins to expand the bandgap initially increases quickly at a rate of $\sim 1 \mathrm{eV} / \AA$, the rate of increase drops as the lattice expands further. Using values for $d E_{g} / d a$ from Figure 6.10 and the measured lattice parameters results in a very modest shift of only $0.02 \mathrm{eV}$, which although a reduction, is far from accounting for the redshift in the GdN measurement.

Continuing on the theme of the lattice reduction in the doped samples, we can consider the effect this may have on the magnitude of the absorption. The transition rate $W_{i \rightarrow f}$ from some initial state $<i \mid$ to final state $\mid f>$ is proportional to the overlap integral

$$
W_{i \rightarrow f} \propto \int \psi_{f}^{*}(\boldsymbol{r}) H^{\prime} \psi_{i}(\boldsymbol{r}) d^{3} \boldsymbol{r}
$$

where $\psi_{f}$ and $\psi_{i}$ are the wave functions of the final and initial states respectively and $H^{\prime} \psi_{i}(\boldsymbol{r})$ is the perturbation caused by the electric field, generally the dipole approximation. It is qualitatively expected that as the lattice in a sample is contracted the overlap between $\psi_{f}$ and $\psi_{i}$ will increase resulting in a larger value for the integral, and an increased absorption at a given frequency. This is at least qualitatively consistent with the data shown for all samples, as the conductive samples, with reduced lattice parameters, have in general a stronger absorption.

\section{Electron-electron interaction}

Now we consider what is known as the re-normalisation of the band structure as a result of doping a material. The bandgap re-normalisation is the decrease in energy of the fundamental bandgap due to electron-electron interactions and electron-ion interactions $[115,116]$. In a simple sense the dopant electrons screen the long-range Coulomb interaction in the lattice. This can then be thought of, in terms of the band structure, as heading from a strongly varying periodic potential towards a more weakly varying potential resulting in a more free-electron like behaviour. The net ef- 
fect of this screening on the bandgap (in a direct gap semiconductor) can be written as

$$
\Delta_{e e}=-\frac{e^{2} k_{f}}{2 \pi^{2} \epsilon_{0} \epsilon}-\frac{e^{2} k_{t f}}{8 \pi \epsilon_{0} \epsilon}\left(1-\frac{4}{\pi} \operatorname{Arctan}\left(k_{f} / k_{t f}\right)\right)
$$

where $k_{f}$ and $k_{t f}$ are the Fermi and Thomas-Fermi wavevectors respectively $[115,116]$. The Thomas-Fermi wavevector is the inverse of the ThomasFermi screening length, which in effect determines the rate at which a potential is screened by conduction electrons in a material, in addition to the permittivity $\epsilon$. The above equation is in the limit that $k_{t f}<k_{f}$ which is generally true in doped semiconductors as $k_{t f} \propto n^{1 / 6}$ and $k_{f} \propto n^{1 / 3}$. Equation 6.2 essentially tells us that as the material is doped with electrons these will act to screen the potential of all ions in the system. Using the measured carrier concentration for the GdN sample and $\epsilon=4$ (determined in the interband region from optical measurements) a value for $\Delta_{e e}$ of $\sim-0.4 \mathrm{eV}$ was found.

Although the combination of the Burstein Moss effect and electronelectron interaction has resulted in a successful description of several moderately doped materials [120,121,122, 123], this simple consideration is not enough to describe the situation in the present GdN sample. The sum of all three contributions to the band shift from the Burstein Moss effect $\Delta_{B M}$ the lattice compression $\Delta_{l}$, and the electron-electron interaction $\Delta_{e e}$ results in a shift to positive energy of about $0.3 \mathrm{eV}$, far from the negative shift seen in Figure 6.7. It is maybe not so surprising that a resolution was not found using these simple considerations. Two of the three considerations (Burstein Moss and electron-electron interaction) considered only static shifts of the band structure, which is very likely not the case. The effect on the band structure of a random concentration of vacancies of 1-5 \% will likely have far more drastic effects than simply a static shift. In any case, these considerations have been instructive and demonstrate how complex even a simply structured material can become when doped at such high levels. 


\section{Increased disorder - lack of $k$ dependence of optical transitions}

It is possible to consider the inclusion of nitrogen vacancies as, in a sense, increasing the disorder in the material. It is important to note that it seems unlikely that these vacancies will increase electron scattering significantly. It is also reasonable they will not have such an effect on the XRD spectra (more than affecting the average lattice spacing between L ions [3]). However, the increased disorder, caused by a few \% of vacancy sites in the lattice, may play a significant role in optical measurements. In a crystalline material Bloch's theorem states that electrons are characterised by a wavevector $\vec{k}$, which is conserved by during optical transitions between two bands. In the case of an amorphous material, the periodicity of the lattice is lost, along with the required conservation of wavevector for an optical transition. In principal, optical transitions across the indirect gap are now allowed $[124,125]$.

It is clear from the XRD measurements in Figure 6.3 that these are not amorphous materials, however, the shift in peak position also shows there is some change to the structure in the more conductive samples. This is most naturally described by the inclusion of randomly spaced nitrogen vacancies which will in some sense reduce the periodicity of the lattice. Instead of considering transitions between any valence and conduction band states it is likely more sensible to now consider transitions from a small range of states $\Delta k$. This will result in a broadening of any features in the absorption, and a shift of the absorption edge to lower energy, as non-vertical translations between the valence and conduction bands are now allowed.

Such an enhancement in the absorption and a transposition to lower energy of the absorption edge has been seen in hydrogenated amorphous Si [117] and other semiconductors [118] as a function of disorder. The increase in absorption and decrease in the optical edge are of similar order to that seen in Figure 6.6. The reduction of the gap in $\mathrm{GdN}$ is from $\sim 1.3 \mathrm{eV}$ in the insulating sample to $\sim 1 \mathrm{eV}$ in the conductive sample, the latter is 
very close to the calculated indirect gap in the paramagnetic state [24].

\section{Existence of impurity states below the conduction band minimum}

Rather than consider the individual effects it is possible to simply calculate, using DFT, the band structure of a GdN lattice which contains some concentration of nitrogen vacancies. A treatment of the electronic structure of GdN, heavily doped to $\sim 3 \%$ with nitrogen vacancies, has previously been undertaken [40] and results in changes to the band structure along with a significant Burstein Moss shift. The main feature of this calculation is the location of three impurity states, these three states correspond to the electrons released from the $\mathrm{N}^{3-}$ ion when a vacancy is created. Two of these states are filled and located in the gap between the conduction and valence bands (one majority spin, one minority). The third (majority spin) impurity state is partially occupied, threading through the bottom of the conduction band.

It is difficult to determine the effects of these impurity states on the optical spectra without a calculation, but, it is possible to consider what may drive the changes. The bottom of the conduction band is significantly altered by the partially filled majority spin impurity state. This raises the Fermi level to within the $\mathrm{Gd} 5 d$ bands to $\sim 0.7 \mathrm{eV}$, very close to what was estimated in the discussion of the Burstein-Moss effect, which considered a comparable number of carriers. The minimum separation between the $\mathrm{N} 2 p$ and $\mathrm{Gd} 5 d$ states also appears reduced to $\sim 0.6 \mathrm{eV}$.

The filled impurity bands now act as new initial states for transitions, although the total density of these states may be on the order of $1 \%$ of the Gd or N states the small width of the band may counteract this somewhat, in terms of a measurable effect on the optical spectra. There are also now transitions from the blue-shifted conduction band minimum to higher bands which may become accessed at around $\sim 0.5 \mathrm{eV}$. The initial states for these transitions are again only on the order of $1 \%$ as dense as the states in the N $2 p$ valence band. However, these may now be tran- 
sitions from a Gd $5 d$ band to another Gd band of higher energy. These transitions, both being on the Gd ion, likely have a much larger overlap and matrix element which may make up for the much smaller density.

It is difficult to compare the calculation [40] to the experimental optical data. One could guess the optical gap in the paramagnetic phase to be close to $0.7 \mathrm{eV}$. This would appear as a redshift on the order of the one observed in the experimental data. The experimental picture is likely complicated even more as the location of the vacancy sites in the material are random, rather than the ordered array used in the calculation. The impurity states will then not form well defined bands and likely not be restricted to strictly vertical transitions on the band structure diagram.

\subsubsection{Low Energy Behaviour of SmN}

We now move to discussing the low energy optical behaviour and implications of Hall effect and resistivity measurements on SmN films. The beginning of this section is centred around the discussion of two films, one insulating and one conductive, the differences between these films and how they relate to expectation (based on the location of the $4 f$ band below the $5 d$ band). This discussion is then extended to the entire series of $\mathrm{SmN}$ films studied.

First we will consider the conductive film shown in red in Figure 6.11. The agreement between the DC conductivity measured via transport measurements (open circles on the plot) and the extrapolation to zero frequency of the free carrier term in the fit to the optical data is very good. The clear free carrier term in the low energy optical conductivity (shown in the red dotted line) also gives a direct measurement of the scattering time $\tau$ in this sample. Using this measured scattering time, the DC conductivity from transport measurements and the carrier concentration from Hall effect measurements, it is possible to determine the effective mass, in a Drude picture, as: 


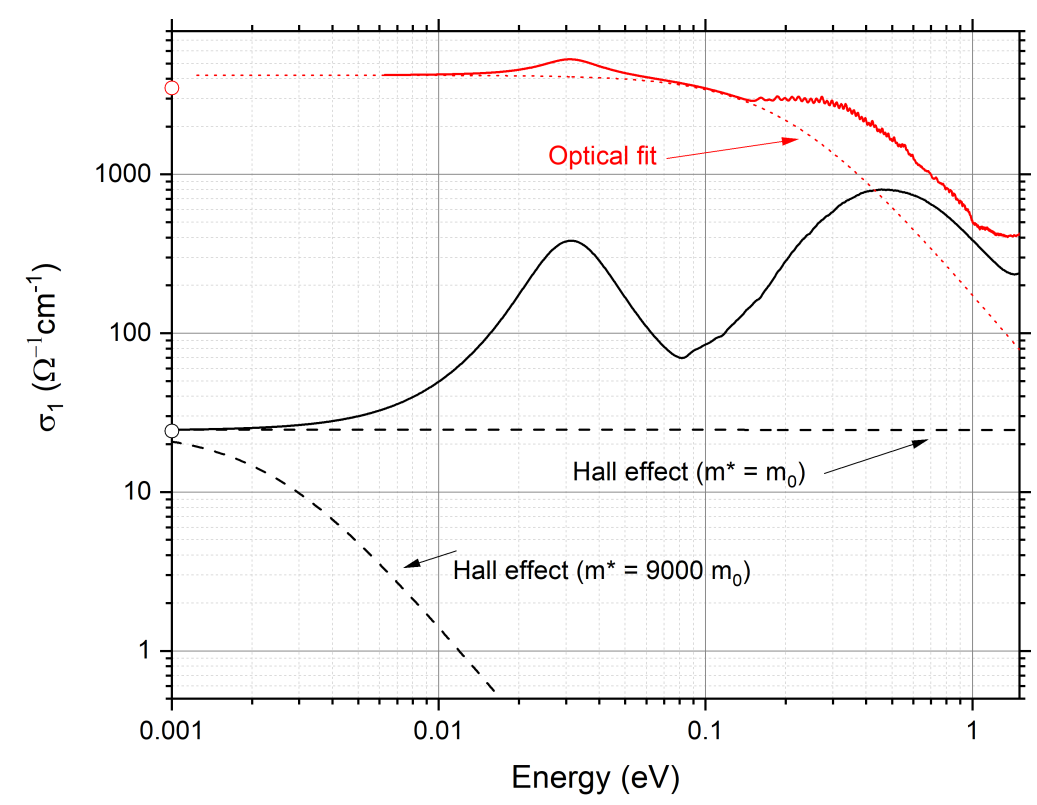

Figure 6.11: Plot of the optical conductivity of a conductive and an insulating $\mathrm{SmN}$ sample, with free carrier terms shown as the dashed series.

$$
m^{*}=\frac{n e^{2} \tau}{\sigma_{D C}} .
$$

This calculation results in a value of $m^{*} \sim 1.1 m_{0}$.

Turning now to the more insulating film, the low energy behaviour of the optical conductivity is dominated by the phonon absorption, so it is not possible to determine a scattering time from the optical measurement. The measurements of DC conductivity and Hall effect are then inconclusive in regards to $m^{*}$ as one can only extract the ratio $\tau / m^{*}$. It is possible to reconcile this and extract a value for $m^{*}$ in this sample by assuming a mean free path $\Lambda$ and writing this using a thermal velocity and scattering time, both of which are functions of the effective mass,

$$
\Lambda=v_{T} \tau, \quad v_{T}=\sqrt{\frac{8 k_{B} T}{\pi m^{*}}}, \quad \tau=\frac{\sigma_{D C} m^{*}}{n e^{2}} .
$$

Now, using the measured values of $\sigma_{D C}$ and $n$ (assuming of course a simple single band Hall effect model) a value for $m^{*}$ can be calculated from a 
sensible choice of $\Lambda$,

$$
m^{*}=\left(\frac{\Lambda n e^{2}}{\sigma_{D C}}\right)^{2} \frac{\pi}{8 k_{B} T} .
$$

For the present investigation a value of $\Lambda=3 \AA$ is chosen as this is the value determined, via the thermal velocity method, for the conductive sample in Figure 6.11. Reasonable upper and lower bounds for the mean free path are the lattice spacing and measured crystallite size in a material. Throughout most $\mathrm{SmN}$ samples the crystallite size changes by no more than $\sim 30 \%$ (15 $\pm 5 \mathrm{~nm}$ ) while the lattice spacing is essentially unchanged on the same scale. Given this it is reasonable, as an initial attempt, to estimate the mean free path (derived from a thermal velocity) to be $\sim 3 \pm 1 \AA$ in all samples. It should finally be noted that applying a free electron model to clearly resistive, non-metallic samples, is inappropriate. The free electron model does, however, appear to describe the conductive sample well, so it is reasonable to expect it will be appropriate at least over some range of conductivities; as such this is explored below.

The above considerations result in an effective mass for the insulating sample shown in Figure 6.11 of $\sim 9000 \mathrm{~m}_{0}$. The plasma frequency and scattering time can then also be renormalised by this same effective mass and used to determine a renormalised free carrier term, this is shown in Figure 6.11, along with the free carrier term corresponding to the free electron mass. Both are consistent with the zero frequency data while the phonon makes it impossible to determine which more accurately represents the experimental optical data (note that the experimental optical data are only reliable to $\sim 0.01 \mathrm{eV}$ ).

The process of renormalising the Hall effect and conductivity measurement of $\tau / m^{*}$ by some mean free path can then be applied to all $\mathrm{SmN}$ films where Hall effect and resistivity measurements have been conducted. The left-hand panel of Figure 6.12 shows the effective mass determined by this analysis for all samples as a function of DC conductivity, assuming again a mean free path $\Lambda=3 \AA$. This plot shows a clear proportionality be- 

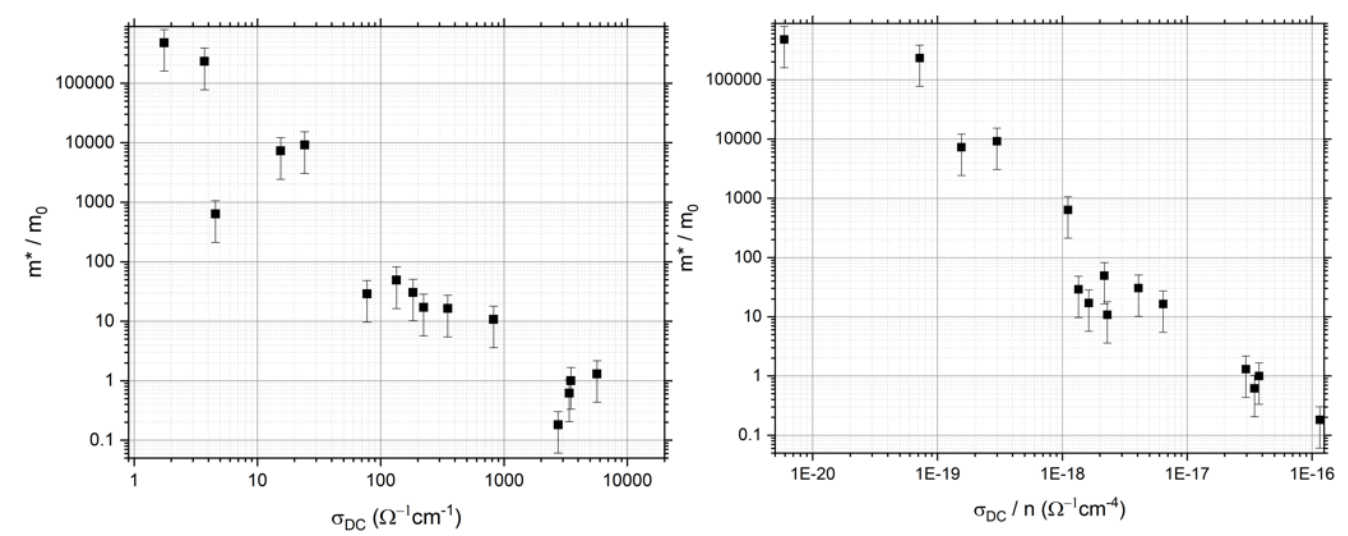

Figure 6.12: Left: Plot of the effective mass as a function of measured DC conductivity for a series of $\mathrm{SmN}$ films. Right Effective mass as a function of the measured DC conductivity normalised by carrier concentration.

tween the effective mass and the conductivity (note that this is the same proportionality that one would see between the DC conductivity and scattering time if a free electron mass was enforced). The DC conductivity for each sample can be further adjusted by the measured carrier concentration, which helps to reduce the spread in the data and is shown in the right-hand panel of Figure 6.12. We now consider a possible physical explanation for the enhanced effective mass in the un-doped samples.

The optical measurements of GdN and NdN described in Section 6.3.2 and the consideration of mechanisms that may contribute to the red shifted optical gap in GdN, showed that there must be rather dramatic changes to the band structure in heavily doped films. This is almost certainly also the case in $\mathrm{SmN}$, however, there is now the additional complication of the $4 f$ levels which exist near the conduction band minimum. The un-doped films, in a sense, fit this expectation in terms of an enhanced effective mass, which is consistent with conduction in a localised $4 f$ band. So the question becomes rather, why does the effective mass appear reduced in doped films?

As discussed previously, the reduced lattice parameter, electron-electron 
interaction and screening effects all likely distort the band structure rather than providing a simple static shift. When considering $\mathrm{SmN}$ there is now the additional $4 f$ band below the $5 d$. It is likely, however, that in $\mathrm{SmN}$ the more localised $4 f$ wave function is not as affected by the above effects as the extended $5 d$, causing the $5 d$ band to fall in energy to a greater degree than the $4 f$.

The estimated gap between the $4 f$ and $5 d$ bands in undoped samples is only $\sim 0.5 \mathrm{eV}$ as shown in Figure 5.14, which is similar to the red-shift shift seen in the GdN and $\mathrm{NdN}$ samples. It now seems possible that in $\mathrm{SmN}$ the distortion to the band structure, caused by doping the material, causes the $5 d$ band to dip below the $4 f$ band. This then results in increased $4 f / 5 d$ hybridisation at the conduction band minimum and transport properties that are more accurately described by the picture of a parabolic conduction band minimum, with a reduced effective mass, in doped samples. This is displayed schematically in Figure 6.13. The left-hand panel of Figure 6.13 shows a schematic conduction band of undoped SmN. The $4 f$ band (red) sits well below the parabolic $5 d$ band (black) as was inferred from optical measurements seen in Chapter 5. In this picture, electron transport is dominated by the heavy mass $4 f$ band. The middle panel now represents a moderately doped sample, both bands have descended, with the $5 d$ band falling in energy faster than the $4 f$. The right-hand panel shows a heavily doped sample, the $5 d$ band now dips below the $4 f$, transport is now dominated by the low mass $5 d$ band.

The above discussion has not mentioned the contributions from impurity bands formed from nitrogen vacancy states. These bands and their hybridisation with the conduction band are likely complex, however, it is possible the low lying $4 f$ of $\mathrm{SmN}$ band actually simplifies matters in regards to the transport properties. In GdN there are two completely filled and one partially filled impurity band. The partially filled band threads through the conduction band, effectively resulting in the partial filling of the Gd $5 d$ band, or Burstein Moss shift. This, naively, cannot be the case in 


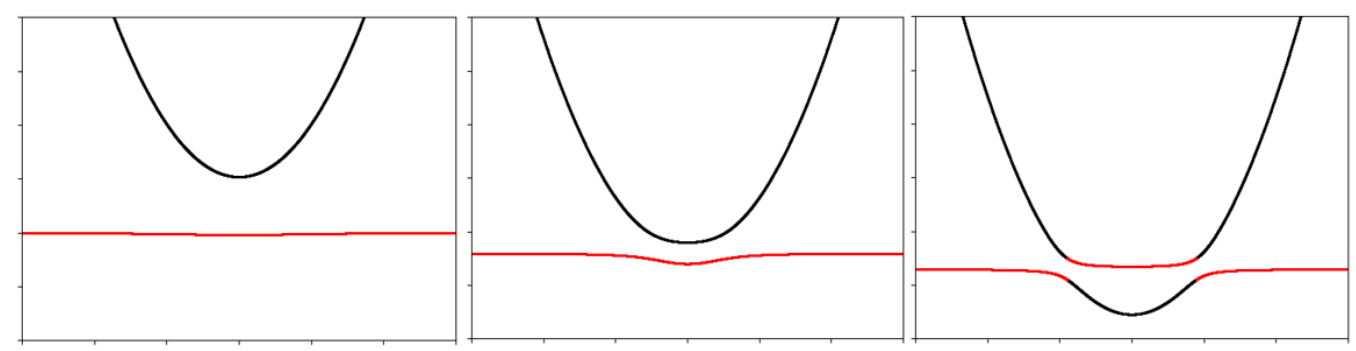

Figure 6.13: Schematic conduction band of SmN showing the $4 f$ (red) and $5 d$ bands (black) only. The left-hand panel shows the un-doped case. As the sample is doped with nitrogen vacancies both bands descend with the $5 d$ falling faster. The right-hand panel show a heavily doped sample where the $5 d$ now falls below the $4 f$, dominating the transport behaviour.

SmN as the narrow $4 f$ band can house $\sim 1 \times 10^{22} \mathrm{~cm}^{-3}$ electrons and thus pins the Fermi energy and the transport properties to this band.

\subsubsection{Magnetic Measurements and Superconductivity}

We now turn to discussing the magnetic properties of doped rare earth nitride films. To begin, measurements of GdN and NdN films are described, these are then discussed in the context of the existing theoretical literature, which is largely limited to GdN. The magnetic state of doped SmN films is then discussed, via measurements of the resistivity, as described in Section 6.2.1. Finally, the observance of superconductivity in $\mathrm{SmN}$ is discussed.

\section{Gadolinium Nitride and Neodymium Nitride}

Measurements of a doped GdN film were discussed in Section 4.4.3 so will not be described in detail here. This film showed a Curie temperature of $\sim 70 \mathrm{~K}$ and clear evidence of two transitions in the temperature derivative of the magnetisation, consistent with previous measurements on heavily doped films [49]. 

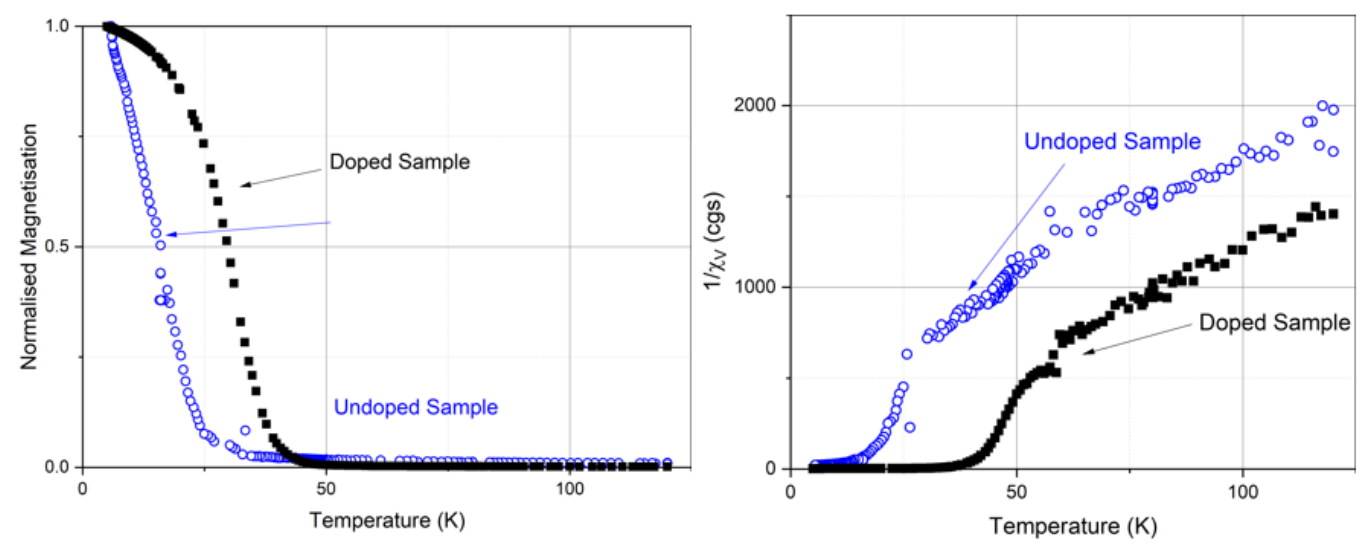

Figure 6.14: Left: Normalised magnetisation of undoped (blue circles) and doped (black squares) NdN films. Right: Inverse susceptibility of the same films in the left hand panel.

Magnetic measurements of both the conductive and insulating $\mathrm{NdN}$ films are now shown in Figure 6.14. The left-hand panel of Figure 6.14 shows the normalised magnetisation of both doped and undoped samples, these measurements were conducted as the samples were cooled in a field of 200 Oe parallel to the sample plane. The doped sample (black squares) shows an increase in the magnetisation near $\sim 40 \mathrm{~K}$, this begins to saturate below $25 \mathrm{~K}$. The magnetisation of the undoped sample only shows a significant upturn below $\sim 25 \mathrm{~K}$ and does not appear to begin to saturate even at the lowest temperatures achieved. The small upturn between $25 \mathrm{~K}$ and $50 \mathrm{~K}$ in the undoped sample is only visible due to the scale of the normalised data and the far from saturated moment.

The inverse susceptibility, shown in the right-hand panel of Figure 6.14, appears relatively linear below $120 \mathrm{~K}$ for each sample before showing a sharp downturn at lower temperatures. This is similar to previous measurements and has been interpreted in terms of the crystal field [43]. The ground state of the $\mathrm{Nd}^{3+}$ ion is split into a doublet and two quartets under the influence of the crystal field, and at higher temperatures, these are all occupied $[6,126]$. At lower temperatures the population of these levels 
changes leading to a deviation from the Curie-Weiss behaviour [43]. The shift in Curie temperature implied from the inverse susceptibility plots shown in Figure 6.14 is quite significant. The doped sample having a Curie temperature of $\sim 40 \mathrm{~K}$ and the undoped $\sim 18 \mathrm{~K}$, when the low-temperature data is considered.

The observed enhancement in Curie temperature has been described, in $\mathrm{GdN}$, in terms of a carrier-mediated exchange [48, 49]. So far calculations considering a carrier-mediated exchange have failed to resolve Curie temperatures above $\sim 60 \mathrm{~K}$ [47]. Calculations have also shown that the magnetic properties of $\mathrm{GdN}$ are very sensitive to the lattice parameter [50]; this is now briefly discussed.

A theoretical study of $\mathrm{GdN}$ considering the nearest $\left(J_{1}\right)$, next nearest $\left(J_{2}\right)$ and third nearest $\left(J_{3}\right)$ neighbours has shown that the three exchange parameters behave quite differently in response to changes in the lattice. As one moves from a compressed to an expanded lattice $J_{1}$ remains negative but becomes smaller in magnitude, $J_{2}$ changes sign from negative to positive and $J_{3}$ retains a small positive value. In effect, this means the largest contribution to the magnetism is a large ferromagnetic exchange between nearest neighbours which weakens as the lattice expands. The second largest contribution is the exchange between next nearest neighbours. This is ferromagnetic at small lattice constants, then weakens and becomes anti-ferromagnetic as the lattice expands.

Two explanations for this behaviour are given. First, at the compressed lattice, the calculated semi-metallic band structure provides a large number of free electrons to aid the exchange, leading to a larger (negative) value for $J_{1}$ and $J_{2}$. As the lattice expands the distance dependent exchange interaction weakens and $J_{1}$ decreases, furthermore, as the band structure becomes more semiconductor like the number of free carriers decreases. There is competition between the carrier-mediated exchange and the anti-ferromagnetic superexchange, this results in a change of sign of $J_{2}$ at larger lattice constants [50]. 
It is true that more heavily doped samples, with reduced lattice parameters, have an enhanced Curie temperature which implies a stronger exchange. The saturated magnetisation of epitaxial GdN samples is also often quoted as $7 \mu_{B}$ per $\mathrm{Gd}^{3+}$, which agrees with expectations from Hund's rules and calculations in the ferromagnetic state. The saturation magnetisation in un-doped samples is (empirically) often much less than $7 \mu_{B}$ per $\mathrm{Gd}^{3+}$ and Curie temperatures closer to $50 \mathrm{~K}$ [4]. It now seems possible that in the compressed (doped) state there is full ferromagnetic exchange ( $J_{1}$ and $J_{2}$ both negative) while in the expanded (undoped) state there is competition between ferromagnetic and anti-ferromagnetic exchange, leading to the discrepancy in both Curie temperature and saturation magnetisation between the two, i.e. a reduced saturation magnetisation and Curie temperature in undoped samples.

\section{Samarium Nitride}

Measurements of the magnetisation in $\mathrm{SmN}$ samples are complicated by the small net moment, which results from the opposition between spin and orbital components in the $4 f$ shell. Although magnetic measurements have been completed on various samples $[58,65]$ they are far from routine, and a systematic study of the magnetic properties of $\mathrm{SmN}$ at various dopant levels has not yet been completed. The temperature-dependent resistivity is a routine measurement on nearly all $\mathrm{SmN}$ samples investigated; this was used to characterise the magnetic state by estimating the Curie temperature in a range of samples, as described in Section 6.2.1

Estimates of the Curie temperature via the resistivity as a function of temperature were used, in part, to construct the phase diagram shown in Figure 6.15. Figure 6.15 shows temperature on the y-axis and conductivity on the x-axis. It should be noted that here conductivity is assumed as a proxy for nitrogen vacancy concentration, i.e. a sample with a low (high) conductivity will have few (many) nitrogen vacancies. Stoichiometric $S m N$ would then appear at the extreme left of the diagram and metallic 


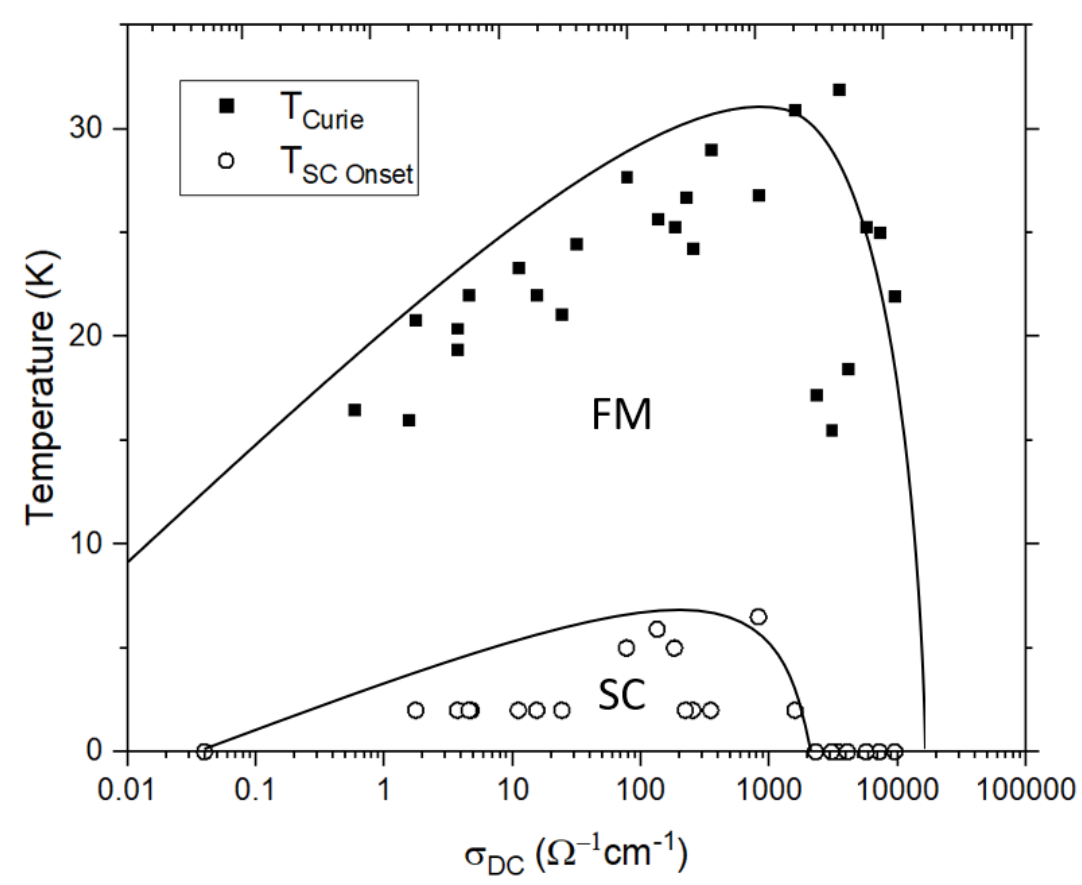

Figure 6.15: Phase diagram showing the low temperature state of $\mathrm{SmN}$ with conductivity (via electrical measurements at $300 \mathrm{~K}$ ) as the order parameter. The squares show measurements of the Curie temperature, the open circles show the onset temperature of a superconducting transition.

Sm at the extreme right. With this in mind Figure 6.15 can be considered. Moving from low to high conductivity in Figure 6.15 it is clear there is a positive correlation between the conductivity and the Curie temperature, before a sharp decline in Curie temperature above $\sim 1000 \Omega^{-1} \mathrm{~cm}^{-1}$. Metallic Sm is anti-ferromagnetic below $14 \mathrm{~K}$ [127] while $\mathrm{SmN}$ is ferromagnetic. If a continuous transition is considered from stoichiometric $\mathrm{SmN}$ to metallic $\mathrm{Sm}$, there must then necessarily a point where the Curie temperature is driven to zero before the Néel temperature begins to rise. This transition from a heavily doped ferromagnetic semiconductor to an antiferromagnetic metal is precisely what is shown as the Curie temperature decreases on the right-hand side of Figure 6.15.

We now move on to discuss the superconducting phase present in SmN. 
Figure 6.15 shows that for all samples within the range of $\sim 1-1000 \Omega^{-1} \mathrm{~cm}^{-1}$ there exists some indication of superconductivity from $2 \mathrm{~K}$ and above, while the onset temperature is suppressed in the more insulating and conductive samples. This feature appears reminiscent of the superconducting domes present in the phase diagrams of many materials, e.g. the high $T_{c}$ Cuprates and heavy Fermion superconductors. It is interesting that the superconducting phase is fully enclosed by the ferromagnetic phase. This is contrary to the Cuprates and heavy Fermion superconductors where the superconducting dome is generally situated over a quantum critical point sandwiched between a non-magnetic and anti-ferromagnetic region. The encapsulation of the superconducting dome by the ferromagnetic region on the phase diagram is then somewhat unique to $\mathrm{SmN}$ and points towards a coexistence of magnetic order and superconductivity.

Furthermore, the onset temperature seems to roughly follow the Curie temperature with the more resistive samples having lower Curie and onset temperatures. The Curie and onset temperatures then seem to dive at roughly the same point near $1000 \Omega^{-1} \mathrm{~cm}^{-1}$. Although far from conclusive, it seems that the same conditions which favour the ferromagnetic state in $\mathrm{SmN}$ also favour the superconducting state, which again points towards a coexistence of ferro-magnetism and superconductivity.

Note should be made of the samples with a quoted onset temperature of $0 \mathrm{~K}$. This indicates that no behaviour relating to a superconducting state was observed above $2 \mathrm{~K}$. It is important to recognise that the changes observed in the magneto-resistance (used in most cases to identify the onset temperature) are often very small. In resistive samples measurements were often inconclusive; this explains the lack of points at low conductivity. On the contrary, measurements were relatively straightforward in conductive samples; thus there is a large grouping of points with an onset temperature of $0 \mathrm{~K}$ above $1000 \Omega^{-1} \mathrm{~cm}^{-1}$.

Finally, we can look at some specific examples of superconductivity in SmN. The left-hand panel of Figure 6.16 shows two measurements of the 

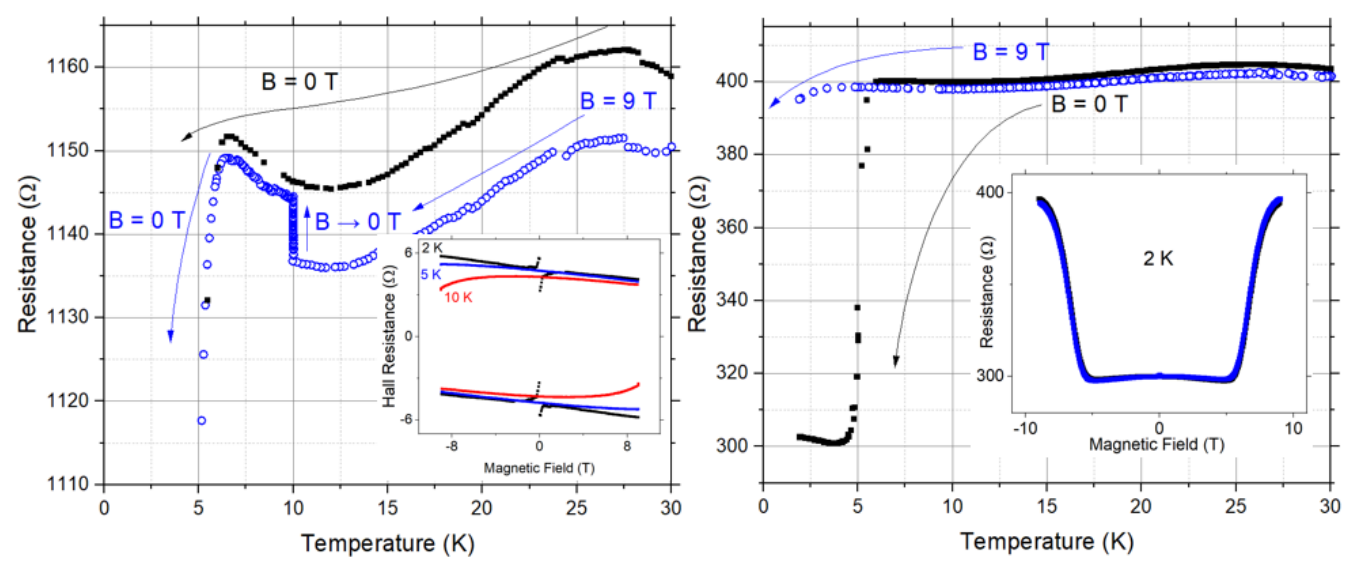

Figure 6.16: Left: Measurement of resistance as a function of temperature in zero field (black) and with variable magnetic field as indicated on the figure (blue) for a SmN sample, which shows the onset of a superconducting transition. The inset shows measurements of the Hall effect at various temperatures. Right Measurements of resistance as a function of temperature in zero field (black) and $9 \mathrm{~T}$ (blue) for a $\mathrm{SmN}$ sample, which shows an incomplete superconducting transition. The inset shows the magneto-resistance at $2 \mathrm{~K}$.

resistivity as a function of temperature of a SmN sample, each measurement with differing magnetic history. The measurement shown in black is simply a zero-field measurement. The sample was cooled in $0 \mathrm{~T}$ from $\sim 40 \mathrm{~K}$, which is above the Curie temperature. Here we can see the peak relating to the ferromagnetic transition centred around $\sim 27 \mathrm{~K}$. Below $12 \mathrm{~K}$ the resistance then begins to increase once more. At $6.5 \mathrm{~K}$ (the highest recorded $T_{o n}$ ) there is a sharp drop in the resistance signalling the onset of superconductivity in this sample. The blue series in the left-hand panel of Figure 6.16 shows the same sample but now in a carefully prepared magnetic state.

By taking advantage of the large coercive field of $\mathrm{SmN}$, it is possible to fully saturate the magnetisation of the sample by cooling the sample in a large field. The field can then be reduced to zero without altering the ferromagnetic alignment. Preparing the sample in such a manner means 
one can attempt to observe the superconducting state in a fully magnetised sample, however, under zero applied field. The sample magnetisation was saturated at $30 \mathrm{~K}$ by a field of $9 \mathrm{~T}$ applied perpendicular to the sample plane. The sample was then cooled in this field to $10 \mathrm{~K}$, as shown in the blue series in Figure 6.16. At this point the sample was held at $10 \mathrm{~K}$, and the field returned to $0 \mathrm{~T}$, this is the vertical increase in resistance shown in the figure at $10 \mathrm{~K}$. It is important to note that due to the large coercive field of SmN when reducing the applied field at $10 \mathrm{~K}$ the spin alignment in the conduction channel is indeed maintained. The sample was then cooled to $2 \mathrm{~K}$ in $0 \mathrm{~T}$. Figure 6.16 shows that preparing the sample in a fully magnetised state has no observable effect on the onset temperature, the initial superconducting transition still occurring at $\sim 6.5 \mathrm{~K}$ (in zero applied field).

Furthermore, we can directly observe the spin imbalance in the conduction channel, and that it is maintained when the field is reduced at $10 \mathrm{~K}$. The anomalous Hall effect is proportional to the spin imbalance in the conduction channel and is shown in the inset, which shows the Hall resistance as a function of applied field at various temperatures. The $10 \mathrm{~K}$ series in the inset is shown in red. There is almost no reduction in the magnitude of the anomalous Hall effect between the field saturated state and the zero-field state. As was said regarding Figure 6.15, this observation may also be relevant when considering the possibility of a triplet pairing mechanism in SmN, as this plot indicates a strong spin in-balance in the conduction channel has no effect on the superconductivity. Furthermore, the inset shows an interesting feature in the $2 \mathrm{~K}$ measurement. Around zero field there appears to be a discontinuity in the Hall measurement, this is currently unexplained.

The right-hand panel of Figure 6.15 shows measurements on a second SmN sample. The black series in the figure shows the resistance as a function of temperature in zero field, a sharp transition can be seen just above $5 \mathrm{~K}$, before the resistance falls to a finite value. It seems here that a portion 
of the sample has entered a superconducting state while other portions do not, signalling an inhomogeneous superconductivity. The blue series in the main panel of the figure shows the same measurement, but now in an applied field (perpendicular to sample plane) of 9 T. Even in this large field the low-temperature data show the onset of superconductivity. The inset to this plot shows the resistance as a function of applied field (perpendicular to sample plane) at $2 \mathrm{~K}$. The superconducting state remains unaffected until a field of $\sim 6 \mathrm{~T}$ is applied. This is now the largest critical field observed in $\mathrm{SmN}$, even surpassing by a factor of three the $\sim 2 \mathrm{~T}$ seen in the SmN / GdN superlattice with field applied perpendicular to the sample plane [58].

\subsection{Conclusions}

Structural, optical, electrical transport and magnetic measurements were conducted on SmN, NdN and GdN samples of varying conductivity. Structural measurements showed that the more conductive samples had a reduced lattice parameter, which is close to the lattice parameters from historical measurements [1]. This implies that the early samples may have been unintentionally doped, which may explain the early confusion regarding the ground state of the rare earth nitrides(i.e. semiconducting or semi-metallic).

Optical measurements found strong free carrier behaviour and a redshifted optical edge in conductive samples. The red-shifted edge was considered in the context of various mechanisms, but no clear description was found. Electrical transport measurements found a significantly reduced resistivity in doped samples and a positive temperature coefficient of resistance in the most doped samples. A preliminary analysis of the transport and optical data in a series of SmN films was completed, which indicates that conductive films have a reduced effective mass when compared to undoped films. 
Magnetic measurements on doped and undoped NdN films have shown an enhanced Curie temperature in doped films, similar to past measurements on GdN. Estimates of the Curie temperature in SmN films (based on resistivity measurements) show the Curie temperature increases in the more conductive films. The Curie temperature then falls in the most conductive films as the material transitions from a doped semiconductor to metallic Sm.

The onset temperature for the superconducting transition in $\mathrm{SmN}$ was found to follow a similar trend to the Curie temperature, in that it increases with the conductivity until $\sim 1000 \Omega^{-1} \mathrm{~cm}^{-1}$ at which point it falls quickly. An analysis on the superconductivity in two $\mathrm{SmN}$ films has shown that both the critical temperature and critical field can be larger than is currently reported in literature [58]. Furthermore, the superconductivity appears unaffected when the film, along with its conduction channel, is prepared in a saturated magnetic state. 


\section{Chapter 7}

\section{Conclusions and Outlook}

In this thesis the electronic structure of $\mathrm{SmN}, \mathrm{NdN}$ and $\mathrm{GdN}$ has been investigated using primarily electrical transport and optical spectroscopy. Previous measurements of the optical properties of the rare earth nitrides have been limited to substrates with optically active phonon modes which have obscured investigation of the low energy behaviours. In the present study optical measurements of both reflection and transmission have been completed, for each material, over the range of $\sim$ meV to $\sim 4 \mathrm{eV}$ by combining low energy measurements on Si and high energy measurements on sapphire substrates. In this way a more complete picture of the optical properties of $\mathrm{SmN}, \mathrm{NdN}$ and $\mathrm{GdN}$ has been obtained.

Low energy measurements on all thee materials studied have found phonon modes in the far infra-red, which are consistent with the calculated values [97]. Low energy measurements on undoped films have found no evidence of free carrier absorption or reflectivity, which provides further evidence in favour of a semiconducting ground state. Measurements in the mid / near infra-red region in SmN and NdN have identified features in the optical conductivity which are consistent with transitions from the valence band into a low lying $4 f$ band, in each material. In $\mathrm{SmN}$ this $4 f$ band was found to be $\sim 0.3 \mathrm{eV}$ above the valence band maximum at $\Gamma$ and to lie below the Sm $5 d$ band. In $\mathrm{NdN}$ the $4 f$ band was found $\sim 1 \mathrm{eV}$ 
above the valence band maximum at $\Gamma$, meeting the $\mathrm{Nd} 5 d$ band at the $\mathrm{X}$ point. Temperature-dependent measurements on $\mathrm{GdN}$ have reproduced previous results of the red-shift of the optical gap in the ferromagnetic phase and extended this work by using the spectra to determine the optical conductivity. Low temperature measurements on $\mathrm{SmN}$ films found only very small changes in the spectra.

Previous studies of the anomalous Hall effect in GdN have already been shown to be consistent with a semi-classical description of the effect [12]. This has been extended by undertaking a study of the anomalous Hall effect in $\mathrm{SmN}$ and $\mathrm{NdN}$. Measurement techniques to overcome experimental challenges relating to the large coercive field in SmN have been developed, which can be applied to other materials with large coercive fields. Measurements of the anomalous Hall effect in $\mathrm{SmN}$ and $\mathrm{NdN}$ are each consistent with a material in which the spin magnetic moment of the conduction electrons is antiparallel to that of the applied field. This is qualitatively expected from the Hund's rules ground state of each material and previous experimental results $[43,52]$.

The anomalous Hall effect was found to be significantly larger in SmN and $\mathrm{NdN}$ when compared to GdN films of a comparable carrier concentration. When interpreted in the semi-classical picture developed previously for $\mathrm{GdN}$, this indicates transport in a $4 f$ conduction channel, which is consistent with the reported optical results. This semi-classical picture was furthermore used to calculate the relative magnitudes of the anomalous Hall effect in $\mathrm{SmN}, \mathrm{NdN}$ and $\mathrm{GdN}$. The calculation gave rough agreement with the experimental results, which again favours the case of $4 f$ conduction in SmN and NdN.

Electrical transport, optical, structural and magnetic measurements were undertaken on heavily doped $\mathrm{SmN}, \mathrm{NdN}$ and GdN films and a preliminary analysis provided. The lattice parameter in doped films was found to be significantly reduced when compared to undoped films, with the reduced lattice parameter matching historical results in the literature [1]. 
This result implies that the earlier studies on the rare earth nitrides suffered from contamination with nitrogen vacancies, which likely led to the confusion regarding the ground state of these materials (i.e. semiconducting or semi-metallic). Transport and optical measurements have shown that films grown with a reduced nitrogen pressure (or increased substrate temperature) appear to be doped with electrons, in line with previous results [108] and calculations [40]. Optical measurements have for the first time seen clear free carrier behaviour in doped rare earth nitrides. In SmN this has been used to measure the scattering time, independently to the Hall effect, and used to estimate the effective mass in a series of $\mathrm{SmN}$ samples. Optical measurements on heavily doped films have furthermore shown a red-shifted absorption edge in GdN, NdN and SmN.

Magnetic measurements conducted on $\mathrm{NdN}$ films have for the first time shown an enhanced Curie temperature in heavily doped films, similar to previous results on $\operatorname{GdN}[48,49]$. The Curie temperature was estimated via resistivity measurements in a series of $\mathrm{SmN}$ films and found to scale with the conductivity.

Finally, a brief investigation of the superconducting state in $\mathrm{SmN}$ has found the superconducting onset temperature scales with the conductivity, in a similar manner to the Curie temperature. Furthermore, the superconducting phase has been found to be completely enclosed by the ferromagnetic phase on a phase diagram of temperature vs. conductivity, here conductivity is used as a proxy for nitrogen vacancy concentration.

In summary:

- The optical properties of SmN, NdN and GdN films have been measured over a more extended region than previous studies.

Phonon modes have been identified for each material, and the locations of the lowest unoccupied majority spin $4 f$ bands in $\mathrm{SmN}$ and NdN have been identified. The semiconducting ground state of undoped films has been confirmed by optical measurements. 
- Measurements of the anomalous Hall effect on SmN, and $\mathrm{NdN}$ films have been completed for the first time.

These measurements show that the majority spin $4 f$ band in both SmN and NdN has an influence over the transport channel, forming either the conduction band minimum or hybridising with the $5 d$ band near the conduction band minimum.

- Heavily doped films have been grown and a preliminary analysis undertaken.

Transport measurements have found a transition from non-metallic like conduction in undoped films to metallic-like conduction in doped films. Optical measurements have found an enhanced free carrier absorption in doped films along with a reduced bandgap. Magnetic measurements have found that the Curie temperature is enhanced in doped films of SmN and $\mathrm{NdN}$ as has previously been seen in GdN.

- The superconducting state of SmN has been investigated.

A phase diagram of $\mathrm{SmN}$ has shown the superconducting phase is entirely enclosed by the ferromagnetic phase. The superconducting transition temperature has found to be suppressed in heavily doped and undoped samples.

\subsection{Recommendations for future work}

- Direct measurement of nitrogen (vacancy) concentration.

Much discussion in this thesis, and in the wider literature regarding the rare earth nitrides, concerns the behaviour of these materials at varying levels of nitrogen vacancy concentration. This is commonly linked to preparation conditions, i.e. the ratio of lanthanide to nitrogen flux. This assumption is entirely reasonable, however, has never been experimentally tested by a direct measurement of the nitrogen 
(vacancy) concentration in a series of films. Such a measurement is possible using nuclear reaction analysis. This will require a series of films to be grown with systematically varying conditions with appropriate substrates and capping layers.

- Optical measurements on LaN and LuN as a function of growth conductions.

The final chapter of this thesis has shown that the optical properties of various rare earth nitrides are very sensitive to preparation conductions, with the assumption that these, in turn, dictate the nitrogen concentration in films. The changes to the band structure are difficult to characterise in materials with $4 f$ levels so close to the conduction and valence band extrema. Optical measurement on a series of $\mathrm{LaN}$ or $\mathrm{LuN}$ would help to provide a clearer picture of the effect of nitrogen vacancies on the band structure of the rare earth nitrides.

- Investigation into the use of YSZ and Si as substrates for [100] epitaxial growth.

There exist reports on the growth of various rare earth nitrides on YSZ and the formation of [100] orientated films. This should be further explored as growth of [100] orientated films would provide a large leap forward and help to align experimental and theoretical efforts.

- Further investigation into the superconducting state of SmN.

Results in Chapter 6 of this thesis have found an interesting relationship between the conductivity of $\mathrm{SmN}$ and the superconducting onset temperature. This, in addition to the observation that the superconducting phase appears to be fully enclosed by the ferromagnetic phase, once again highlights the importance of studying the nature of superconductivity in $\mathrm{SmN}$. 
- Investigation of the rare earth nitrides in the context of strongly correlated electron systems.

In this thesis, the location of the $4 f$ levels in $\mathrm{SmN}$ and $\mathrm{NdN}$ has been found as, or in close proximity to, the conduction band minimum. The $4 f$ bands have also been shown to influence the transport properties of these materials. The simple structure, control of the location of the $4 f$ levels in the conduction and valence bands, along with the ability to tune the Fermi energy by doping, highlight the rare earth nitrides as an ideal platform for the investigation of strongly correlated electron systems. 


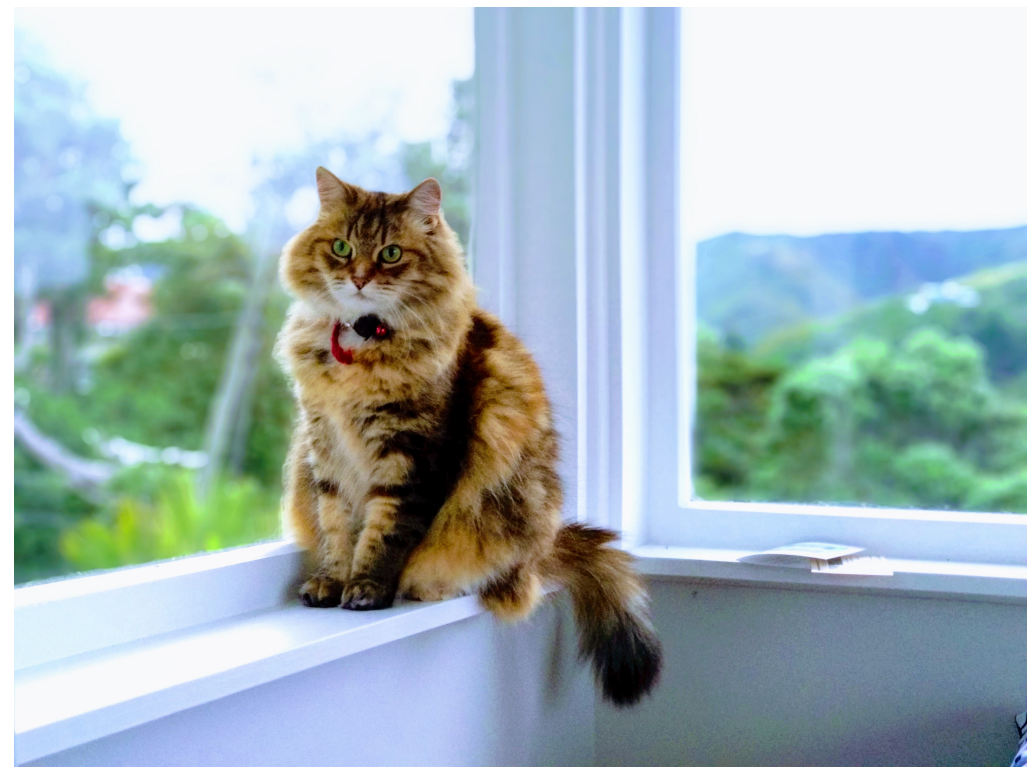




\section{Bibliography}

[1] F. Natali, B. Ruck, N. Plank, H. Trodahl, S. Granville, C. Meyer, and W. Lambrecht, "Rare-earth mononitrides," Progress in Materials Science, vol. 58, no. 8, pp. 1316 -1360, 2013.

[2] P. Larson, W. R. L. Lambrecht, A. Chantis, and M. vanSchilfgaarde, "Electronic structure of rare-earth nitrides using the LSDA+U approach: Importance of allowing $4 \mathrm{f}$ orbitals to break the cubic crystal symmetry," Phys. Rev. B, vol. 75, no. 4, p. 045114, 2007.

[3] K. Senapati, M. G. Blamire, and Z. H. Barber, "Spin-filter Josephson junctions," Nat. Mater., vol. 10, no. 10, p. 849, 2011.

[4] K. Khazen, H. J. von Bardeleben, J. L. Cantin, A. Bittar, S. Granville, H. J. Trodahl, and B. J. Ruck, “Ferromagnetic resonance study of gdn thin films with bulk and extended lattice constants," Phys. Rev. B, vol. 74, p. 245330, Dec 2006.

[5] A. Shaib, F. Natali, J. R. Chan, F. Ullstad, W. F. Holmes-Hewett, J. D. Miller, B. J. Ruck, and H. J. Trodahl, “Coexisting structural phases in the catalytically driven growth of rock salt GdN," Mater. Res. Express, vol. 7, p. 046404, apr 2020.

[6] D. P. Schumacher and W. E. Wallace, "Magnetic characteristics of some lanthanide nitrides," Inorganic Chemistry, vol. 5, no. 9, pp. 1563-1567, 1966. 
[7] G. Busch, "Magnetic properties of rare-earth compounds," Journal of Applied Physics, vol. 38, no. 3, pp. 1386-1394, 1967.

[8] F. Hulliger, "Magnetic properties of the rare earth pnictides," Journal of Magnetism and Magnetic Materials, vol. 8, no. 3, pp. 183 - 205, 1978.

[9] F. Hulliger, "Chapter 33 rare earth pnictides," in Non-Metallic Compounds - II, vol. 4 of Handbook on the Physics and Chemistry of Rare Earths, pp. 153 - 236, Elsevier, 1979.

[10] S. Granville, B. J. Ruck, F. Budde, A. Koo, D. J. Pringle, F. Kuchler, A. R. H. Preston, D. H. Housden, N. Lund, A. Bittar, G. V. M. Williams, and H. J. Trodahl, "Semiconducting ground state of GdN thin films," Phys. Rev. B, vol. 73, p. 235335, Jun 2006.

[11] F. Ullstad, G. Bioletti, J. R. Chan, A. Proust, C. Bodin, B. J. Ruck, J. Trodahl, and F. Natali, "Breaking molecular nitrogen under mild conditions with an atomically clean lanthanide surface," ACS Omega, vol. 4, no. 3, pp. 5950-5954, 2019.

[12] H. J. Trodahl, F. Natali, B. J. Ruck, and W. R. L. Lambrecht, “Carriercontrolled anomalous Hall effect in an intrinsic ferromagnetic semiconductor," Phys. Rev. B, vol. 96, p. 115309, Sep 2017.

[13] A. H. Macdonald, P. Schiffer, and N. Samarth, "Ferromagnetic semiconductors: Moving beyond (Ga,Mn)As," Nat. Mater., vol. 4, no. 3, p. $195,2005$.

[14] F. Natali, B. Ludbrook, J. Galipaud, N. O. V. Plank, S. Granville, A. Preston, B. L. Do, J. Richter, I. Farrell, R. Reeves, S. Durbin, H. J. Trodahl, and B. Ruck, "Epitaxial growth and properties of GdN, EuN and SmN thin films," Phys. Status Solidi (c), vol. 9, 032012.

[15] F. Natali, N. O. Plank, J. Galipaud, B. J. Ruck, H. J. Trodahl, F. Semond, S. Sorieul, and L. Hirsch, "Epitaxial growth of GdN on sili- 
con substrate using an AlN buffer layer," Journal of Crystal Growth, vol. 312, no. 24, pp. 3583-3587, 2010.

[16] J. Chan, S. Vézian, J. Trodahl, M. Khalfioui, B. Damilano, and F. Natali, "Temperature-Induced Four-Fold-on-Six-Fold Symmetric Heteroepitaxy, Rocksalt SmN on Hexagonal AlN," Cryst. Growth Des, vol. 16, 092016.

[17] P. K. Muduli, A. Pal, and M. G. Blamire, "Crossover from diffusive to tunneling regime in $\mathrm{NbN} / \mathrm{DyN} / \mathrm{NbN}$ ferromagnetic semiconductor tunnel junctions," Phys. Rev. B, vol. 89, p. 094414, Mar 2014.

[18] J. Miller, F. Ullstad, H. J. Trodahl, B. Ruck, and F. Natali, "Vertical transport and tunnelling in rare-earth nitride heterostructures," Nanotechnology, 2020.

[19] J. F. McNulty, E. M. Anton, B. J. Ruck, F. Natali, H. Warring, F. Wilhelm, A. Rogalev, M. M. Soares, N. B. Brookes, and H. J. Trodahl, "Twisted phase of the orbital-dominant ferromagnet SmN in a GdN/SmN heterostructure," Physical Review B - Condensed Matter and Materials Physics, vol. 91, no. 17, pp. 1-7, 2015.

[20] J. F. McNulty, E.-M. Anton, B. J. Ruck, M. Suzuki, M. Mizumaki, and H. J. Trodahl, "Tunable magnetic exchange springs in semiconductor GdN/NdN superlattices," Phys. Rev. B, vol. 100, p. 094441, Sep 2019.

[21] W. F. Holmes-Hewett, R. G. Buckley, B. J. Ruck, F. Natali, and H. J. Trodahl, "Optical spectroscopy of SmN: Locating the $4 f$ conduction band," Phys. Rev. B, vol. 99, p. 205131, May 2019.

[22] W. F. Holmes-Hewett, R. G. Buckley, B. J. Ruck, F. Natali, and H. J. Trodahl, " $4 f$ conduction in the magnetic semiconductor NdN," Phys. Rev. B, vol. 100, p. 195119, Nov 2019. 
[23] M. Topsakal and R. Wentzcovitch, "Accurate projected augmented wave (PAW) datasets for rare-earth elements (RE=La-Lu)," Computational Materials Science, vol. 95, pp. 263 - 270, 2014.

[24] H. J. Trodahl, A. R. H. Preston, J. Zhong, B. J. Ruck, N. M. Strickland, C. Mitra, and W. R. L. Lambrecht, "Ferromagnetic redshift of the optical gap in GdN," Phys. Rev. B, vol. 76, no. 8, p. 085211, 2007.

[25] C. Mitra and W. R. L. Lambrecht, "Magnetic exchange interactions in the gadolinium pnictides from first principles," Phys. Rev. B, vol. 78, p. 134421 , Oct 2008.

[26] M. D. Johannes and W. E. Pickett, "Magnetic coupling between nonmagnetic ions: $\mathrm{Eu}^{3+}$ in $\mathrm{EuN}$ and EuP," Phys. Rev. B, vol. 72, p. 195116, Nov 2005.

[27] J. H. Richter, B. J. Ruck, M. Simpson, F. Natali, N. O. V. Plank, M. Azeem, H. J. Trodahl, A. R. H. Preston, B. Chen, J. McNulty, K. E. Smith, A. Tadich, B. Cowie, A. Svane, M. van Schilfgaarde, and W. R. L. Lambrecht, "Electronic structure of EuN: Growth, spectroscopy, and theory," Phys. Rev. B, vol. 84, p. 235120, Dec 2011.

[28] T. Cheiwchanchamnangij and W. R. L. Lambrecht, "Fully opposite spin polarization of electron and hole bands in dyn and related band structures of gdn and hon," Phys. Rev. B, vol. 92, p. 035134, Jul 2015.

[29] N. N. Som, V. H. Mankad, S. D. Dabhi, A. Patel, and P. K. Jha, "Magnetic behavior study of samarium nitride using density functional theory," J. Magn. Magn. Mater., vol. 448, pp. 186 - 191, 2018.

[30] C. Morari, F. Beiuşeanu, I. Di Marco, L. Peters, E. Burzo, S. Mican, and L. Chioncel, "Magnetism and electronic structure calculation of SmN," J. Phys. Condens. Matter, vol. 27, no. 11, p. 115503, 2015. 
[31] A. Hasegawa and A. Yanase, "Energy Band Structures of GdPnictides," Journal of the Physical Society of Japan, vol. 42, no. 2, pp. 492-498, 1977.

[32] C. M. Aerts, P. Strange, M. Horne, W. M. Temmerman, Z. Szotek, and A. Svane, "Half-metallic to insulating behavior of rare-earth nitrides," Phys. Rev. B, vol. 69, p. 045115, Jan 2004.

[33] P. Larson and W. R. L. Lambrecht, "Electronic structure of Gd pnictides calculated within the LSDA +U approach," Phys. Rev. B, vol. 74, p. 085108, Aug 2006.

[34] H. Yamada, T. Fukawa, T. Muro, Y. Tanaka, S. Imada, S. Suga, D.-X. Li, and T. Suzuki, "XPS and X-BIS Studies of Gd Monopnictides," Journal of the Physical Society of Japan, vol. 65, no. 4, pp. 1000-1004, 1996.

[35] P. Wachter, Handbook on the Physics and Chemistry of Rare Earths. Elsevier, Amsterdam, 1979.

[36] A. N. Chantis, M. van Schilfgaarde, and T. Kotani, "Quasiparticle self-consistent $G W$ method applied to localized $4 f$ electron systems," Phys. Rev. B, vol. 76, p. 165126, Oct 2007.

[37] T. Cheiwchanchamnangij, Applications of the Quasiparticle Selfconsistent GW Method. PhD thesis, Case Western Reserve University, 2014.

[38] F. Leuenberger, A. Parge, W. Felsch, K. Fauth, and M. Hessler, "GdN thin films: Bulk and local electronic and magnetic properties," Phys. Rev. B, vol. 72, p. 014427, Jul 2005.

[39] L. Degiorgi, W. Bacsa, and P. Wachter, "Electronic structure of $\mathrm{YbN}$," Phys. Rev. B, vol. 42, pp. 530-539, Jul 1990. 
[40] A. Punya, T. Cheiwchanchamnangij, A. Thiess, and W. R. L. Lambrecht, "First-principles study of nitrogen vacancies in GdN," MRS Proc., vol. 1290, no. 4, p. 4, 2011.

[41] N. W. Ashcroft and N. D. Mermin, Solid State Physics. Holt-Saunders, 1976.

[42] J. F. McNulty, B. J. Ruck, and H. J. Trodahl, “On the ferromagnetic ground state of smn," Phys. Rev. B, vol. 93, p. 054413, Feb 2016.

[43] E.-M. Anton, J. F. McNulty, B. J. Ruck, M. Suzuki, M. Mizumaki, V. N. Antonov, J. W. Quilty, N. Strickland, and H. J. Trodahl, “NdN: An intrinsic ferromagnetic semiconductor," Phys. Rev. B, vol. 93, p. 064431, Feb 2016.

[44] D. B. Ghosh, M. De, and S. K. De, "Electronic, magnetic, and optical properties of Gd monopnictides: An LDA + U study," Phys. Rev. B, vol. 72, p. 045140, Jul 2005.

[45] C.-G. Duan, R. F. Sabiryanov, W. N. Mei, P. A. Dowben, S. S. Jaswal, and E. Y. Tsymbal, "Magnetic ordering in Gd monopnictides: Indirect exchange versus superexchange interaction," Applied Physics Letters, vol. 88, no. 18, p. 182505, 2006.

[46] A. Mauger and C. Godart, "The magnetic, optical, and transport properties of representatives of a class of magnetic semiconductors: The europium chalcogenides," Physics Reports, vol. 141, no. 2, pp. 51 $-176,1986$.

[47] A. Sharma and W. Nolting, "Additional carrier-mediated ferromagnetism in GdN," Phys. Rev. B, vol. 81, p. 125303, Mar 2010.

[48] N. O. V. Plank, F. Natali, J. Galipaud, J. H. Richter, M. Simpson, H. J. Trodahl, and B. J. Ruck, "Enhanced Curie temperature in N-deficient GdN," Appl. Phys. Lett., vol. 98, p. 112503, 022011. 
[49] F. Natali, B. J. Ruck, H. J. Trodahl, D. L. Binh, S. Vezian, B. Damilano, Y. Cordier, F. Semond, and C. Meyer, "Role of magnetic polarons in ferromagnetic GdN," Phys. Rev. B, vol. 87, p. 035202, Jan 2013.

[50] C.-G. Duan, R. F. Sabiryanov, J. Liu, W. N. Mei, P. A. Dowben, and J. R. Hardy, "Strain Induced Half-Metal to Semiconductor Transition in GdN," Phys. Rev. Lett., vol. 94, p. 237201, Jun 2005.

[51] C. Morari, F. Beiuşeanu, I. Di Marco, L. Peters, E. Burzo, S. Mican, and L. Chioncel, "Magnetism and electronic structure calculation of SmN," J. Phys. Condens. Matter, vol. 27, no. 11, p. 115503, 2015.

[52] E.-M. Anton, B. J. Ruck, C. Meyer, F. Natali, H. Warring, F. Wilhelm, A. Rogalev, V. N. Antonov, and H. J. Trodahl, “Spin/orbit moment imbalance in the near-zero moment ferromagnetic semiconductor SmN," Phys. Rev. B, vol. 87, p. 134414, Apr 2013.

[53] J. Ziman, The Theory of Transport Phenomena in Solids. Oxford University Press, 1960.

[54] M. Fox, Optical Properties of Solids. Oxford University Press, 2001.

[55] F. Ullstad, G. Bioletti, J. Chan, A. Proust, C. Bodin, B. J. Ruck, H. J. Trodahl, and F. Natali, "Breaking molecular nitrogen under mild conditions with an atomically clean lanthanide surface," ArXiv, vol. x, p. x, Dec 2018.

[56] M. Azeem, B. J. Ruck, D. Binh, H. Warring, N. Strickland, A. Koo, V. Goian, S. Kamba, and H. J. Trodahl, "Optical Response of DyN," J. Appl. Phys, vol. 113, p. 203509, 072013.

[57] H. Warring, B. J. Ruck, J. F. McNulty, E.-M. Anton, S. Granville, A. Koo, B. Cowie, and H. J. Trodahl, "YbN: An intrinsic semiconductor with antiferromagnetic exchange," Phys. Rev. B, vol. 90, p. 245206, Dec 2014. 
[58] E.-M. Anton, S. Granville, A. Engel, S. V. Chong, M. Governale, U. Zülicke, A. G. Moghaddam, H. J. Trodahl, F. Natali, S. Vézian, and B. J. Ruck, "Superconductivity in the ferromagnetic semiconductor samarium nitride," Phys. Rev. B, vol. 94, p. 024106, Jul 2016.

[59] W. F. Holmes-Hewett, F. H. Ullstad, B. J. Ruck, F. Natali, and H. J. Trodahl, "Anomalous Hall effect in SmN: Influence of orbital magnetism and $4 f$-band conduction," Phys. Rev. B, vol. 98, p. 235201, Dec 2018.

[60] H. Yoshitomi, S. Kitayama, T. Kita, O. Wada, M. Fujisawa, H. Ohta, and T. Sakurai, "Optical and magnetic properties in epitaxial GdN thin films," Phys. Rev. B, vol. 83, p. 155202, 042011.

[61] M. Azeem, "Spin-split joint density of states in GdN," Chin. Phys. Lett., vol. 33, p. 027501, feb 2016.

[62] M. Azeem, "Optical Properties of Rare Earth Nitrides," no. June 2013, 2013.

[63] M. Azeem, “On the optical energy gap of smn," Chin. J. Phys., vol. 56, no. 5, pp. $1925-1930,2018$.

[64] B. Ludbrook, I. Farrell, M. Kübel, B. Ruck, A. Preston, J. Trodahl, L. Ranno, R. Reeves, and S. Durbin, "Growth and properties of epitaxial GdN," Journal of Applied Physics, vol. 106, p. 063910, 102009.

[65] C. Meyer, B. J. Ruck, J. Zhong, S. Granville, A. R. H. Preston, G. V. M. Williams, and H. J. Trodahl, "Near-zero-moment ferromagnetism in the semiconductor SmN," Phys. Rev. B, vol. 78, p. 174406, Nov 2008.

[66] B. J. Ruck, H. J. Trodahl, J. H. Richter, J. C. Cezar, F. Wilhelm, A. Rogalev, V. N. Antonov, B. D. Le, and C. Meyer, "Magnetic state of eun: X-ray magnetic circular dichroism at the eu $M_{4,5}$ and $L_{2,3}$ absorption edges," Phys. Rev. B, vol. 83, p. 174404, May 2011. 
[67] D. Le Binh, B. J. Ruck, F. Natali, H. Warring, H. J. Trodahl, E.-M. Anton, C. Meyer, L. Ranno, F. Wilhelm, and A. Rogalev, "Europium nitride: A novel diluted magnetic semiconductor," Phys. Rev. Lett., vol. 111, p. 167206, Oct 2013.

[68] H. Warring, Device Applications of Rare Earth Nitrides. PhD thesis, Victoria University of Wellington, 2016.

[69] C. Hadenfeldt, H. Jacobs, and R. Juza Z. anorg, allg. Chem., vol. 379, 1970.

[70] W. H. Bragg and W. L. Bragg, "The reflection of x-rays by crystals," Proceedings of the Royal Society of London. Series A, Containing Papers of a Mathematical and Physical Character, vol. 88, no. 605, pp. 428-438, 1913.

[71] J. B. Nelson and D. P. Riley, "An experimental investigation of extrapolation methods in the derivation of accurate unit-cell dimensions of crystals," Proceedings of the Physical Society, vol. 57, pp. 160177, may 1945.

[72] A. L. Patterson, "The scherrer formula for $x$-ray particle size determination," Phys. Rev., vol. 56, pp. 978-982, Nov 1939.

[73] R. J. Gambino, T. R. McGuire, H. A. Alperin, and S. J. Pickart, “Magnetic properties and structure of GdN and GdN1-xOx," Jour. Appl. Phys., vol. 41, no. 3, pp. 933-934, 1970.

[74] L. van der Pauw, "A method of measuring specific resistivity and hall effect of discs of arbitrary shape," Philips Research Reports, vol. 13, pp. 1-9, 1958.

[75] E. H. Hall, "On a new action of the magnet on electric currents," American Journal of Mathematics, vol. 2, pp. 287-292, sep 1879. 
[76] E. H. Hall, "On the "rotational coefficient" in nickel and cobalt," Proceedings of the Physical Society of London, vol. 4, pp. 325-342, aug 1880.

[77] A. Kundt, "Das hall'sche phänomen in eisen, kobalt und nickel," Annalen der Physik, vol. 285, no. 6, pp. 257-271, 1893.

[78] J. Smit, "The spontaneous hall effect in ferromagnetics i," Physica, vol. 21, no. 6, pp. $877-887,1955$.

[79] J. Smit, "The spontaneous hall effect in ferromagnetics ii," Physica, vol. 24, no. 1, pp. $39-51,1958$.

[80] L. Berger, "Side-jump mechanism for the hall effect of ferromagnets," Phys. Rev. B, vol. 2, pp. 4559-4566, Dec 1970.

[81] R. Karplus and J. M. Luttinger, "Hall effect in ferromagnetics," Phys. Rev., vol. 95, p. 1154, Sep 1954.

[82] N. Nagaosa, J. Sinova, S. Onoda, A. H. MacDonald, and N. P. Ong, “Anomalous Hall effect," Rev. Mod. Phys., vol. 82, p. 1539, May 2010.

[83] M. V. Berry, "Quantal phase factors accompanying adiabatic changes," Proceedings of the Royal Society of London, vol. 392, 1984.

[84] D. Xiao, M. C. Chang, and Q. Niu, "Berry phase effects on electronic properties," Reviews of Modern Physics, vol. 82, no. 3, pp. 1959-2007, 2010.

[85] N. P. Ong and W.-L. Lee, "Geometry and the anomalous Hall effect in ferromagnets," in Foundations of Quantum Mechanics in the Light of New Technology ISQM-Tokyo '05 (S. Ishioka and K. Fujikawa, eds.), pp. 121-126, June 2006.

[86] E. Clementi and D. L. Raimondi, "Atomic screening constants from scf functions," The Journal of Chemical Physics, vol. 38, no. 11, pp. 2686-2689, 1963. 
[87] E. Clementi, D. L. Raimondi, and W. P. Reinhardt, "Atomic screening constants from scf functions. ii. atoms with 37 to 86 electrons," The Journal of Chemical Physics, vol. 47, no. 4, pp. 1300-1307, 1967.

[88] N. Nagaosa, X. Z. Yu, and Y. Tokura, "Gauge fields in real and momentum spaces in magnets: monopoles and skyrmions," Philosophical Transactions of the Royal Society A: Mathematical, Physical and Engineering Sciences, vol. 370, no. 1981, pp. 5806-5819, 2012.

[89] Y. Taguchi, Y. Oohara, H. Yoshizawa, N. Nagaosa, and Y. Tokura, "Spin chirality, Berry phase, and anomalous Hall effect in a frustrated ferromagnet," Science, vol. 291, no. 5513, pp. 2573-2576, 2001.

[90] P. Bruno, V. K. Dugaev, and M. Taillefumier, "Topological Hall effect and Berry phase in magnetic nanostructures," Phys. Rev. Lett., vol. 93, p. 096806, Aug 2004.

[91] C. M. Hurd, The Hall effect in metals and alloys. New York: Plenum, 1972.

[92] B. M. Ludbrook, G. Dubuis, A. H. Puichaud, B. J. Ruck, and S. Granville, "Nucleation and annihilation of skyrmions in $\mathrm{Mn}_{2} \mathrm{CoAl}$ observed through the topological Hall effect," Sci. Rep., vol. 7, p. $13620,2017$.

[93] N. Sclar, "Properties of rare-earth nitrides," Journal of Applied Physics, vol. 35, no. 5, pp. 1534-1538, 1964.

[94] M. P. Singh, C. S. Thakur, K. Shalini, S. Banerjee, N. Bhat, and S. A. Shivashankar, "Structural, optical, and electrical characterization of gadolinium oxide films deposited by low-pressure metalorganic chemical vapor deposition," Journal of Applied Physics, vol. 96, no. 10, pp. 5631-5637, 2004. 
[95] R. Vidyasagar, T. Kita, T. Sakurai, and H. Ohta, "Electronic transitions in gdn band structure," J. Appl. Phys., vol. 115, no. 20, p. 203717, 2014.

[96] A. R. H. Preston, B. J. Ruck, W. R. L. Lambrecht, L. F. J. Piper, J. E. Downes, K. E. Smith, and H. J. Trodahl, “Electronic band structure information of GdN extracted from x-ray absorption and emission spectroscopy," Appl. Phys. Lett., vol. 96, no. 3, p. 032101, 2010.

[97] S. Granville, C. Meyer, A. R. H. Preston, B. M. Ludbrook, B. J. Ruck, H. J. Trodahl, T. R. Paudel, and W. R. L. Lambrecht, “Vibrational properties of rare-earth nitrides: Raman spectra and theory," Phys. Rev. B, vol. 79, p. 054301, 022009.

[98] H. Ehrenreich, H. R. Philipp, and B. Segall, "Optical properties of aluminum," Phys. Rev., vol. 132, pp. 1918-1928, Dec 1963.

[99] A. B. Kuzmenko, "Kramers-kronig constrained variational analysis of optical spectra," Rev. Sci. Instrum, vol. 76, no. 8, p. 083108, 2005.

[100] J. D. Jackson, Classical electrodynamics; 2nd ed. New York, NY: Wiley, 1975.

[101] O. S. Heavens, "Optical properties of thin films," Reports on Progress in Physics, vol. 23, pp. 1-65, jan 1960.

[102] R. Hull, Properties of crystalline silicon. London: INSPEC, the Institution of Electrical Engineers, 1999.

[103] C. D. Salzberg and J. J. Villa, "Infrared refractive indexes of silicon germanium and modified selenium glass," J. Opt. Soc. Am., vol. 47, pp. 244-246, Mar 1957.

[104] I. H. Malitson, "Refraction and dispersion of synthetic sapphire," J. Opt. Soc. Am., vol. 52, pp. 1377-1379, Dec 1962. 
[105] J. Kischkat, S. Peters, B. Gruska, M. Semtsiv, M. Chashnikova, M. Klinkmüller, O. Fedosenko, S. Machulik, A. Aleksandrova, G. Monastyrskyi, Y. Flores, and W. T. Masselink, "Mid-infrared optical properties of thin films of aluminum oxide, titanium dioxide, silicon dioxide, aluminum nitride, and silicon nitride," Appl. Opt., vol. 51, pp. 6789-6798, Oct 2012.

[106] C. Mitra and W. R. L. Lambrecht, "Calculated interband optical transition spectra of GdN," Phys. Rev. B, vol. 78, p. 195203, Nov 2008.

[107] T. Maity, H. J. Trodahl, F. Natali, B. J. Ruck, and S. Vézian, "Electron transport in heavily doped GdN," Phys. Rev. Materials, vol. 2, p. 014405, Jan 2018.

[108] J. R. Chan, M. Al Khalfioui, S. Vézian, J. Trodahl, B. Damilano, and F. Natali, "Effect of the growth temperature and nitrogen precursor on the structural and electrical transport properties of SmN thin films," MRS Advances, vol. 2, no. 3, p. 165-171, 2017.

[109] R. Kaufmann and O. Meyer, "Determination of static displacements around non-metal vacancies in $\mathrm{NbN}_{1 c}$ and $\mathrm{TiC}_{1 c}$ by channeling," Solid State Communications, vol. 51, no. 7, pp. $539-543,1984$.

[110] P. P. Craig, W. I. Goldburg, T. A. Kitchens, and J. I. Budnick, “Transport properties at critical points: The resistivity of nickel," Phys. Rev. Lett., vol. 19, pp. 1334-1337, Dec 1967.

[111] M. E. Fisher and J. S. Langer, "Resistive anomalies at magnetic critical points," Phys. Rev. Lett., vol. 20, pp. 665-668, Mar 1968.

[112] V. Novák, K. Olejník, J. Wunderlich, M. Cukr, K. Výborný, A. W. Rushforth, K. W. Edmonds, R. P. Campion, B. L. Gallagher, J. Sinova, and T. Jungwirth, "Curie Point Singularity in the Temperature Derivative of Resistivity in (Ga,Mn)As," Phys. Rev. Lett., vol. 101, p. 077201, Aug 2008. 
[113] A. Travlos, N. Salamouras, and N. Boukos, "Growth of rare earth silicides on silicon," Journal of Physics and Chemistry of Solids, vol. 64, no. 1, pp. $87-93,2003$.

[114] T. S. Moss, "The interpretation of the properties of indium antimonide," Proceedings of the Physical Society. Section B, vol. 67, pp. 775782 , oct 1954.

[115] J. C. Inkson, "The effect of electron interaction on the band gap of extrinsic semiconductors," Journal of Physics C: Solid State Physics, vol. 9, pp. 1177-1183, apr 1976.

[116] R. Abram, G. Rees, and B. Wilson, "Heavily doped semiconductors and devices," Advances in Physics, vol. 27, no. 6, pp. 799-892, 1978.

[117] G. Cody, T. Tiedje, B. Abeles, T. Moustakas, B. Brooks, and Y. Goldstein, "Disorder and the optical-absorption edge of hydrogenated amorphous silicon," Phys. Rev. Lett., vol. 42, 111981.

[118] S. K. O'Leary, "Optical absorption, disorder, and the disorderless limit in amorphous semiconductors," Applied Physics Letters, vol. 72, no. 11, pp. 1332-1334, 1998.

[119] P. Giannozzi, S. Baroni, N. Bonini, M. Calandra, R. Car, C. Cavazzoni, D. Ceresoli, G. L. Chiarotti, M. Cococcioni, I. Dabo, A. D. Corso, S. de Gironcoli, S. Fabris, G. Fratesi, R. Gebauer, U. Gerstmann, C. Gougoussis, A. Kokalj, M. Lazzeri, L. Martin-Samos, N. Marzari, F. Mauri, R. Mazzarello, S. Paolini, A. Pasquarello, L. Paulatto, C. Sbraccia, S. Scandolo, G. Sclauzero, A. P. Seitsonen, A. Smogunov, P. Umari, and R. M. Wentzcovitch, “QUANTUM ESPRESSO: a modular and open-source software project for quantum simulations of materials," Journal of Physics: Condensed Matter, vol. 21, p. 395502, sep 2009. 
[120] A. Steinhoff, M. Rösner, F. Jahnke, T. O. Wehling, and C. Gies, “Influence of excited carriers on the optical and electronic properties of mos $_{2}$," Nano Letters, vol. 14, no. 7, pp. 3743-3748, 2014.

[121] Y. Park, S. Han, R. Taylor, C. Chan, B. Reid, Y. Jo, N. Kim, H. Im, and K. Kim, "Interplay between many body effects and coulomb screening in optical bandgap of atomically thin $\operatorname{mos}_{2}, "$ Nanoscale, vol. 9, 052017.

[122] D. C. Reynolds, D. C. Look, and B. Jogai, "Combined effects of screening and band gap renormalization on the energy of optical transitions in $\mathrm{ZnO}$ and GaN," Journal of Applied Physics, vol. 88, no. 10, pp. 5760-5763, 2000.

[123] A. Walsh, J. L. F. Da Silva, and S.-H. Wei, "Origins of band-gap renormalization in degenerately doped semiconductors," Phys. Rev. B, vol. 78, p. 075211, Aug 2008.

[124] C. Kittel, Introduction to Solid State Physics. John Whiley and Sons, 1968.

[125] J. M. Ziman, Models of Disorder. Cambridge: Cambridge University Press, 1979.

[126] K. Lea, M. Leask, and W. Wolf, "The raising of angular momentum degeneracy of $f$-electron terms by cubic crystal fields," Journal of Physics and Chemistry of Solids, vol. 23, no. 10, pp. 1381 - 1405, 1962.

[127] J. M. Lock, "The magnetic susceptibilities of lanthanum, cerium, praseodymium, neodymium and samarium, from $1.5 \mathrm{k}$ to $300 \mathrm{k}$," Proceedings of the Physical Society. Section B, vol. 70, pp. 566-576, jun 1957. 